\title{
AN INVESTIGATION INTO THE CHARACTERISTICS AND SOURCES OF LIGHT EMISSION AT DEEP-SEA HYDROTHERMAL VENTS
}

\author{
By \\ Sheri N. White \\ B.S.A.A.E., Purdue University \\ (1993) \\ SUBMITTED IN PARTIAL FULFILLMENT OF THE REQUIREMENTS \\ FOR THE DEGREE OF \\ DOCTOR OF PHILOSOPHY \\ at the \\ MASSACHUSETTS INSTITUTE OF TECHNOLOGY \\ and the \\ WOODS HOLE OCEANOGRAPHIC INSTITUTION \\ June 2000 \\ (c) 2000 Sher1 N. White \\ All rights reserved.
}

The author hereby grants to MIT and WHOI permission to reproduce paper and electronic opies of this thesis mo whole or in part, and to distribute them publicly.

Signature of Aưthor,

Joint Program in Oceanography

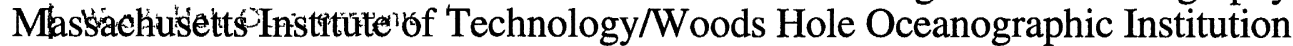

April 28, 2000

Certified by

Alan D. Chave

Thesis Supervisor

Accepted by

Timothy Grove

Chair, Joint Committee for Marine Geology \& Geophysics Massachusetts Institute of Technology/Woods Hole Oceanographic Institution 


\title{
AN INVESTIGATION INTO THE ChaRaCTERISTICS AND SOURCES OF LIGHT EMISSION AT DEEP-SEA HYDROTHERMAL VENTS
}

\author{
by \\ SHERI N. WHITE \\ Submitted to the Department of Earth, Atmospheric and Planetary Sciences \\ on April 28, 2000 in partial fulfillment of the \\ requirements for the Degree of Doctor of Philosophy in \\ Marine Geophysics
}

\begin{abstract}
A spectral camera (ALISS - Ambient Light Imaging and Spectral System) was used to image ambient light from high-temperature vents at $9^{\circ} \mathrm{N}$ East Pacific Rise and the Juan de Fuca Ridge during 1997 and 1998 Alvin dive cruises. ALISS is a low-light digital camera with custom-designed optics. A set of nine lenses, each covered by an individual bandpass filter (50 and $100 \mathrm{~nm}$ nominal bandwidths), allows vents to be imaged in nine wavelength bands simultaneously spanning the range of $400-1000 \mathrm{~nm}$. Thus, both spatial and spectral information are obtained.

ALISS was used to image three types of vents: black smokers, flange pools, and beehives. The primary source of light is thermal radiation due to the high temperature of the hydrothermal fluid $\left(\sim 350^{\circ} \mathrm{C}\right)$. This light is dominant at wavelengths greater than $700 \mathrm{~nm}$. At flange pools, where the fluid is relatively stable, only thermal radiation is present. Black smokers and beehives, however, are subject to mixing with ambient seawater $\left(2^{\circ} \mathrm{C}\right)$ leading to mineral precipitation. Data from these types of vents show the existence of non-thermal, temporally varying light in the $400-700 \mathrm{~nm}$ region. This light is probably caused by mechanisms related to mixing and precipitation, such as chemiluminescence, crystalloluminescence and triboluminescence.
\end{abstract}

Thesis Supervisor: Alan D. Chave

TITLE: Senior Scientist, Department of Applied Ocean Physics \& Engineering, Woods Hole Oceanographic Institution 


\section{ACKNOWLEDGMENTS}

A number of people have supported me and assisted me both professionally and personally throughout my tenure in the Joint Program. I owe a huge debt of gratitude to all of them for making the past six years possible, bearable, and (often) a lot of fun.

First and foremost, I thank my advisor Alan Chave. He took a chance on an aerospace engineer and helped me to become an oceanographer. He provided me with numerous opportunities to attend meetings (even when I had nothing to present), to go on cruises (just to get sea-going experience), and to dive in the submersible Alvin (an experience I never dreamed I would have).

Due to the interdisciplinary nature of my thesis, by committee members have been vitally important: George Reynolds (physics), Greg Ravizza (geology/chemistry), Colleen Cavanaugh (biology), Tina Voelker (chemistry), and Sandy Williams (engineering). George Reynolds helped me enormously in understanding the physics of light emission and optics, and in keeping track of the details. (He tried his best to make a physicist out of me). Susan Humphris was one of my generals project advisors as well as chair of my thesis committee. I learned a lot by working with her — both about hydrothermal processes, and about how to be a scientist (not just a woman scientist). Cindy Van Dover was the first to discover vent light, so without her insight and persistence none of this work would have ensued. Although they were not on my committee, discussions with Tony Tyson, Alan Walton, Meg Tivey, and Karen Von Damm help to increase my understanding of CCD technology, optics, hydrothermal systems, and chemistry.

The ALISS camera was a great instrument to work with and it performed beautifully. A number of people helped to build and maintain her: Bruce Truax and Chris Gaal designed the optics; Eric Gaidos and the Palomar Group at Caltech provided assistance with calibration, and Eric helped with the image processing routines; Rod Catanach designed, built, and modified (at sea) the ALISS frame. John Bailey not only helped me by building and maintaining the instrumentation I used, nor just by being invaluable at sea, he also gave me the support I needed during particularly stressful cruises and showed me that no matter what the problem, I could "improvise, adapt, and overcome." He also taught me everything I know about soldering, o-rings, and making things simple and "dummy proof".

The crew of the R/VAtlantis and the Alvin group were vital in collecting in the data for this work, and I owe them all a great deal of gratitude. I especially thank BLee Williams, Matt Heintz, and Steve Falutico for piloting me to hydrothermal fields at the bottom of the ocean - the most amazing places I have every visited. My fellow scientists

on the Legacy Cruise, HotTimes Cruise, and Endeavour Cruise both helped to collect the data in this thesis, and helped to keep me sane during the time I spent at sea. Special 
thanks go to Alison Bray, Rob Evans, Cindy Van Dover, Colleen Cavanaugh, Greg Ravizza, Dave Brenner, Johnathan Robinson, and Paul Field (who kept my feet warm in the sub).

Of course, graduate school is about more that just research... My incoming class (WHOI '94) provided support, camaraderie, and friendship. I am especially grateful for the wonderful housemates I have had: Ann Pearson, Liz Kujawinski, and Steve Jayne. My officemates, fellow G\&G students, and miscellaneous Woods Hole folks have been patient, supportive, and fun (especially Gary Jaraslow, Dana Stuart, Sooze Carter ( \& Franzie, Henry, and Gus), Ben Gutierrez (\& Erin Galvin), Mike Braun, Jen Georgen, Carrie Tuit, Mark Behn, and Shannon "troll" McDaniel). The Education Office (especially Julia (\& Jayne), Marsha, Melissa, and Stella) provided me with a place for daily visits away from my office. And TSG (and the Capt'n Kidd) gave me the strength (or escape) I needed to get through the last few months.

I would not have survived grad school without the sport of rugby. The MIT Women's Rugby Team and coaches (1995-1998) introduced me to the sport which became an obsession. Playing rugby provided me with an outlet for stress, an appreciation for scars and bruises, and a group of good friends (especially the Old Girls - Katy Q, Kiwi Karen, Ali, Vanessa, Natasha, and Marianne; and the youngsters - Susan and Radika).

My best friend "Jack" (Christie Schoeder Iacomini) was the one who talked me into grad school. She managed my to stay my friend over thousands of miles, despite my poor correspondence, and though changes in my life and hers. I really value the support she gave and continues to give me.

Meghan Hanawalt (and Bronski, Norma, and Pee) reminded me that there is more to life than science... and rugby. She enriched my life with her love, spirit, and humor. I feel incredibly lucky to have her in my life.

My parents, Drs. Terry and Olivia White, by their example, set high goals for me to strive for. I inherited a fascination with engineering and how things work from my dad, and a love for science from my mom. But from them I also acquired a respect for cultures, an interest in history, and a love of travel. I am grateful for the love and support they gave me throughout my life, and in particular during the past six years.

I would like to dedicate this thesis to my grandparents, Howard Clinton and Alberta Louise White. They both passed away in my fifth year, and so are unable to see me become Dr. White. But they always provided me with love and support no matter what I was doing (striving to be an astronaut, traveling the world, or diving to the bottom of the ocean in Alvin)... and no matter how much it scared them. 
Wär' nicht das Auge sonnenhaft, Die Sonne könnt' es nie erblicken

Were the eye not attuned to the Sun, The sun could never be seen by it.

- Goethe, 1805 


\section{TABLE OF CONTENTS}

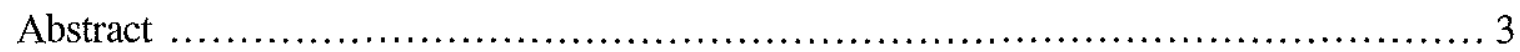

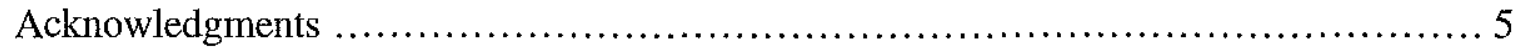

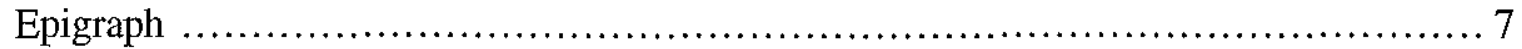

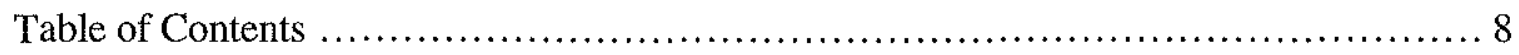

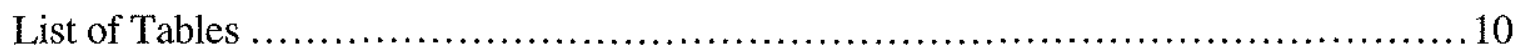

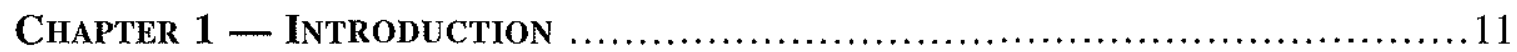

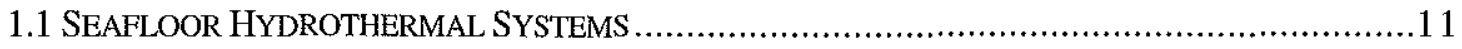

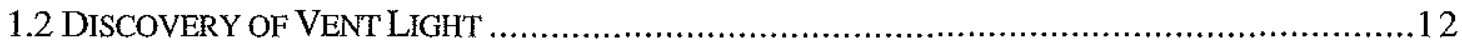

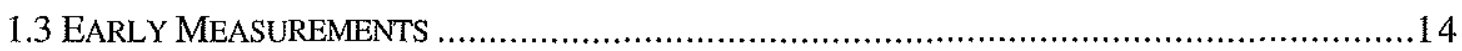

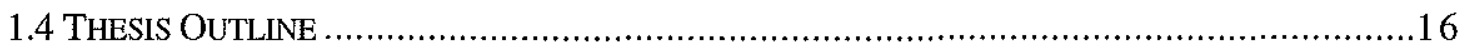

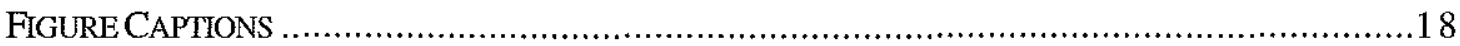

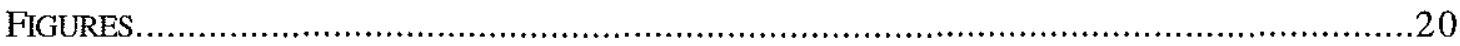

Chapter 2 - Possible Sources of Vent Light .............................. 27

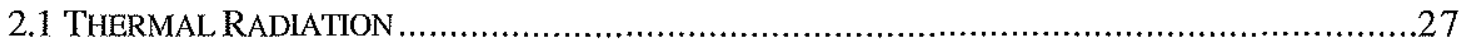

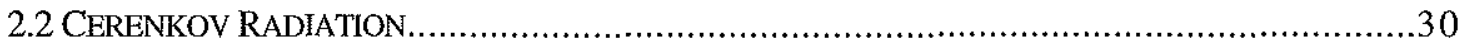

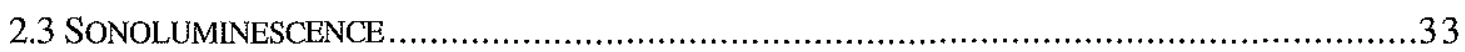

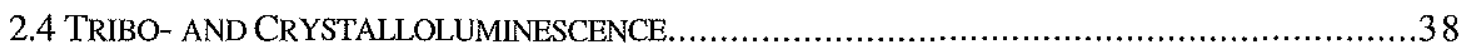

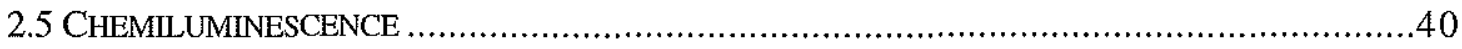

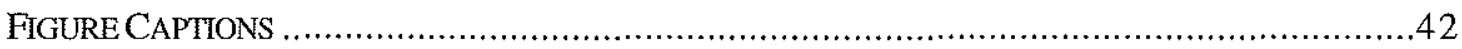

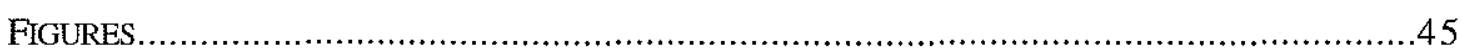

Chapter 3 - ALISS (Ambient Light Imaging and Spectral System) .....55

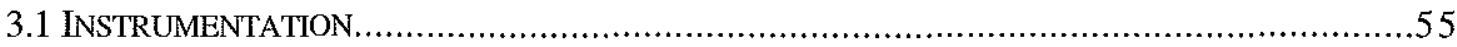

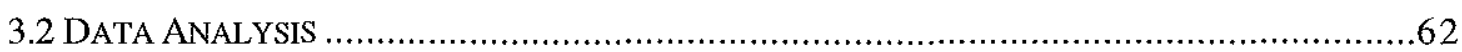

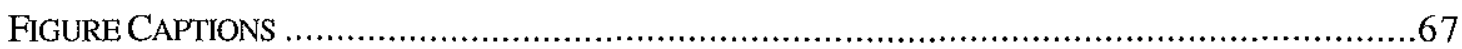

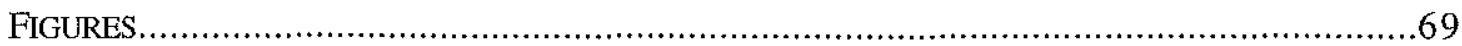


Chapter 4 - Physical Properties of Sea Water \& Vent Fluid ..............79

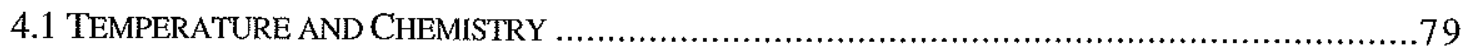

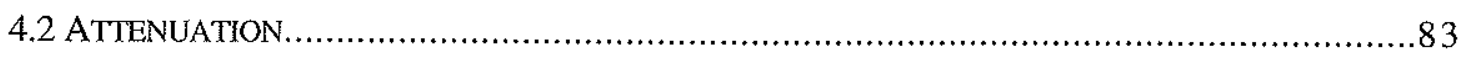

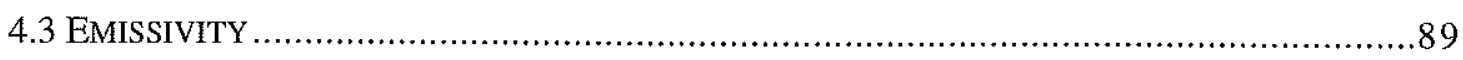

TABLES …

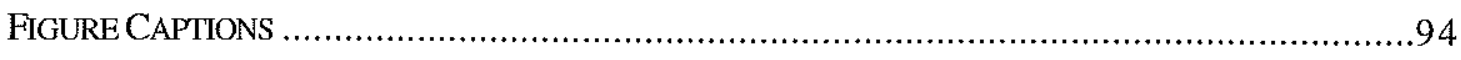

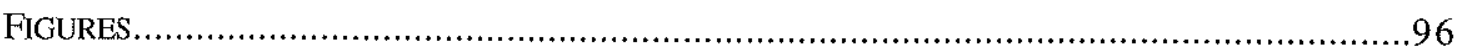

Chapter 5 - Ambient Imaging at $9^{\circ}$ N East Pacific Rise and Endeavour SEgment, JuAn de Fuca RIDGe .................. 101

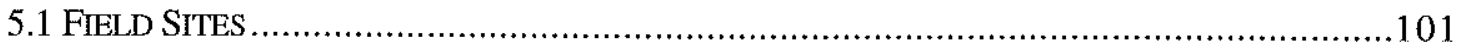

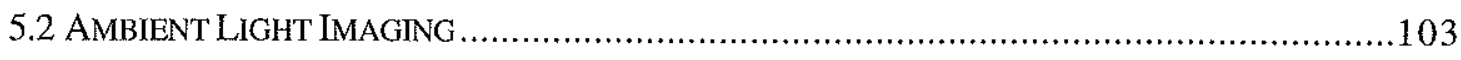

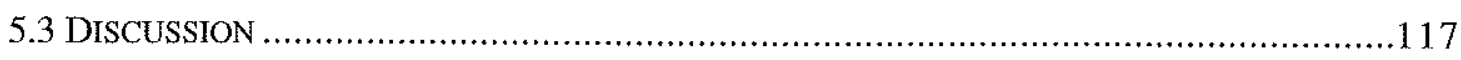

TABLES …

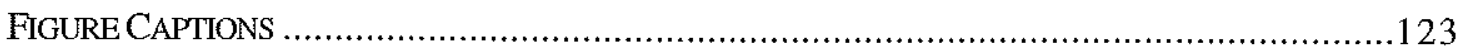

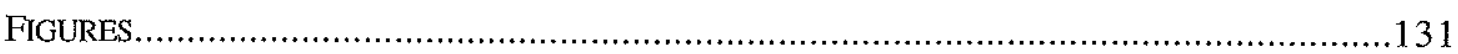

Chapter 6 - Conclusions, Implications, \& Future Work ................ 163

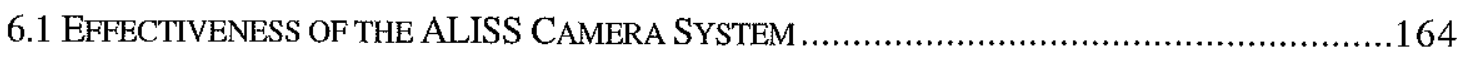

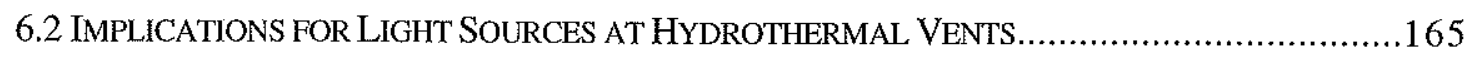

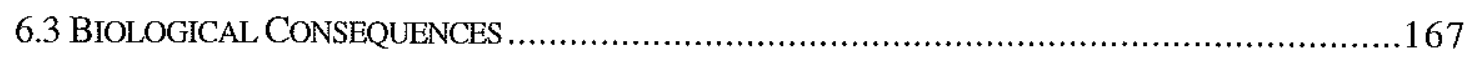

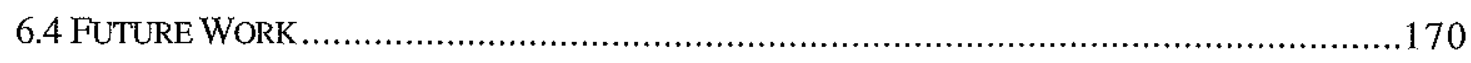

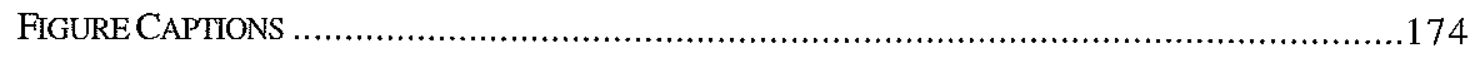

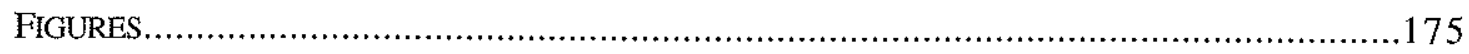

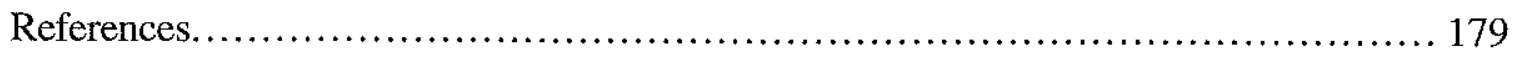

Biographical Note ............................................................ 188 


\section{List OF TABLES}

\section{Chapter 4}

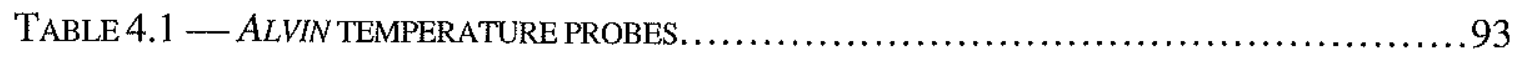

TABle 4.2 — Endeavour Vent Chemistry (1994) ............................. 93

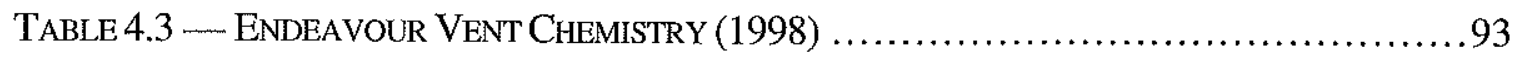

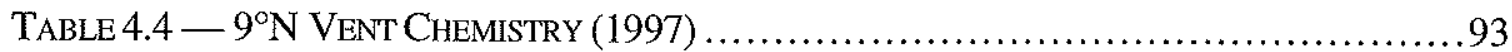

\section{Chapter 5}

TABLE 5.1 - ENDEAVOUR VENTS IMAGED BY ALISS............................. 121

TABle 5.2 - East Pacific Rise Vents IMAged by ALISS ......................... 121

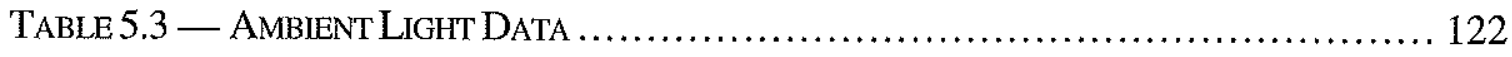




\section{ChaPter 1 - INTROdUCTION}

\subsection{Seafloor Hydrothermal Systems}

The $75,000 \mathrm{~km}$ long mid-ocean ridge (MOR) is a divergent plate margin where upwelling magma generates new oceanic crust. Hydrothermal circulation is an integral component of crustal formation on the seafloor. Seawater penetrates into young crust where it is heated and interacts with the surrounding rock [Alt, 1995]. Altered hydrothermal fluid then rises buoyantly to the seafloor where it exits as diffuse lowtemperature flow [e.g., Edmond et al., 1979], or focused, high-temperature venting [e.g., Von Damm et al., 1995a]. Hydrothermal circulation effects the chemical composition of both seawater and oceanic crust [Edmond et al., 1979], and is responsible for about $34 \%$ of heat lost from oceanic crust [Stein and Stein, 1994].

Hydrothermal systems have been observed on slow-, intermediate-, and fastspreading mid-ocean ridges throughout the world [Fornari and Embley, 1995] (Figure 1.1). These include the TAG Hydrothermal Field at $26^{\circ} 08^{\prime} \mathrm{N}$ on the slow-spreading MidAtlantic Ridge [Rona et al., 1986]; the Main Endeavour Field (MEF) at $47^{\circ} 57^{\prime} \mathrm{N}$ on the intermediate-spreading Juan de Fuca Ridge [Delaney et al., 1992]; and the Venture Hydrothermal Field at $9^{\circ} 50^{\prime} \mathrm{N}$ on the fast-spreading East Pacific Rise [Haymon et al., 1991].

The injection of hot hydrothermal fluid into cold seawater results in the precipitation of minerals and the formation of sulfide deposits. The morphology of these deposits, and the chemistry of the exiting fluids can vary among vent sites [Tivey, 1995]. Three types of high-temperature vents will be discussed in this thesis - black smokers, flange pools, and beehives (Figure 1.2). Tivey [1995] describes the formation of each of these types of structures: 


\section{Black Smokers}

Black smoker vents consist of a sulfide structure ("chimney") with a central conduit through which high-temperature $\left(\sim 350^{\circ} \mathrm{C}\right)$ fluid passes (Figure 1.2A). As the hydrothermal fluid exits the chimney it mixes with entrained ambient seawater causing mineral precipitation in the rising plume. The presence of minerals in the plume gives it a dark, smoky appearance — hence the name "black smoker".

\section{Flange Pools}

The same high-temperature $\left(\sim 350^{\circ} \mathrm{C}\right)$ fluid that exits black smokers, can also be trapped beneath flanges (Figure 1.2B) along the sides of large sulfide structures (such as those found on the Juan de Fuca ridge). The fluids pooled beneath these flanges are stably stratified, forming a sharp interface with the ambient $\left(\sim 2^{\circ} \mathrm{C}\right)$ seawater below through which minimal mixing occurs [Delaney et al., 1992].

\section{Beehives}

Beehive structures (or "diffusers") are bulbous features with high porosity through which hydrothermal fluids percolate (Figure 1.2C). The structure itself is primarily a matrix of anhydrite, with lesser amounts of pyrite, chalcopyrite, sphalerite, and wurtzite. Clear fluids less than $350^{\circ} \mathrm{C}$ emanate from the sides of theses structures [Tivey, 1995].

\subsection{Discovery OF VeNT Light}

The search for light in deep-sea environments was prompted by the discovery of a unique species of shrimp living at high-temperature hydrothermal vents on the Mid-Atlantic Ridge. This shrimp, Rimicaris exoculata, which lives in swarms on the sides of sulfide 
chimneys was first described by Williams and Rona [1986]. Despite the fact that this species of vent shrimp lacks normal eyes and eyestalks (hence the name exoculata - Latin, meaning "without eyes"), it is capable of detecting light with large paired photoreceptors located beneath the transparent carapace on the shrimp's dorsal side [Van Dover et al., 1989] (Figure 1.3A). These "eyes" (apparently derived from normal shrimp eyes) are uniquely adapted to detect very low levels of light by maximizing photon absorption. These adaptations include its large size (about $0.5 \%$ of the animal's body volume), large entrance aperture (more than $26 \mathrm{~mm}^{2}$ ) and a matrix of white diffusing cells which reflect incoming photons upward into the photoreceptors [O'Neill et al.; 1995]. Studies of the pigment within the eye show it to be rhodopsin — the light absorbing pigment contained in the eyes of humans as well as other animals - with a concentration much greater than in typical shallow-water species. Measurements of the pigment's absorption between 300 and $800 \mathrm{~nm}$ reveal maximum absorption at $500 \mathrm{~nm}$ (Figure 1.3B) [Van Dover et al., 1989].

At the time that this novel photoreceptor was discovered, the deep-sea environment was thought to be void of light other than small flashes of bioluminescence. Since "normal" shrimp eyes are capable of seeing bioluminescence, it was unclear why $R$. exoculata had developed such a unique photoreceptor. Considering the environment in which the shrimp lived, it was suggested that the high-temperature hydrothermal vents were emitting light [Van Dover et al., 1989]. Calculations by Pelli and Chamberlain [1989] suggested that the high-temperature $\left(\sim 350^{\circ} \mathrm{C}\right)$ fluid exiting these vents generates enough thermal radiation to be detected by the shrimp. Although the spectrum of a $350^{\circ} \mathrm{C}$ black body peaks in the infrared (at $\sim 4600 \mathrm{~nm}$ ), its tail extends into the visible region of the spectrum. (This will be discussed further in Chapter 2.) 


\subsection{Early Measurements}

The existence of light emission at deep-sea hydrothermal vents was first verified in 1988 [Smith and Delaney, 1989; Van Dover et al., 1994a]. During a dive of the submersible Alvin to a vent on the Endeavour Segment of the Juan de Fuca Ridge (Alvin dive 2074), an image was taken of the vent with a charge-coupled device (CCD) camera with all of Alvin's external lights secured. This image (Figure 1.4) clearly shows light emanating from the vent orifice and decreasing in intensity with distance above the orifice. During a subsequent dive (Alvin dive 2109), glass filters were placed in front of the camera during the imaging to obtain a rough spectrum of the vent light [Smith and Delaney, unpublished]. The six filters (blue, green, yellow, orange, red, and visible) had broad bandwidths and were not infrared (IR) blocked (which is important when looking at thermal radiation which peaks in the infrared). Thus, a rigorous determination of spectral variation was not possible.

In an effort to obtain spectral data on the light emanating from vents, a simple photometer called Optical Properties Underwater Sensor (OPUS) was built at the Woods Hole Oceanographic Institution (WHOI) [Van Dover et al., 1996]. The first generation OPUS, housed in a cylindrical (10 cm diameter) lucite pressure-case, consisted of four Hamamatsu silicon photodiodes ( $33 \mathrm{~mm}^{2}$ active area), each of which was covered by an individual Omega Optical interference filter. The filters, $100 \mathrm{~nm}$ nominal bandwidth and IR blocked, were centered at 700, 800, 900 and $1000 \mathrm{~nm}$ - in the far red/near infrared region of the spectrum where thermal radiation is expected to be strong. The OPUS instrument was deployed in 1993 at high-temperature hydrothermal vents on the Mid-Atlantic Ridge (Snake Pit site - $23^{\circ} 22^{\prime} \mathrm{N}, 44^{\circ} 57^{\prime} \mathrm{W}$ ) and the East Pacific Rise (the Hole-to-Hell area $\left.9^{\circ} 50^{\prime} \mathrm{N}, 104^{\circ} 17.5^{\prime} \mathrm{W}\right)$. OPUS measurements confirmed the existence of long-wavelength light $(>700 \mathrm{~nm})$ consistent with thermal radiation. However, the data also suggested the 
possibility of light in the visible region of the spectrum which exceeds that expected from thermal radiation alone ( $\sim 19$ times what is expected in the $650-750 \mathrm{~nm}$ range), and temporal variations which could not be explained by thermal radiation from a constant temperature source (Figure 1.5) [Van Dover et al., 1996].

A second generation of OPUS (Figure 1.6) consisted of two OPUS housings containing a total of eight photodiodes and a series of $100 \mathrm{~nm}$ bandwidth interference filters (IR blocked) that covered the spectrum from 450 to $1000 \mathrm{~nm}$ in $50 \mathrm{~nm}$ increments [White et al., 1996a]. This instrument was deployed at a number of vents in the Venture Hydrothermal Field on the East Pacific Rise $\left(9^{\circ} 30^{\prime} \mathrm{N}\right.$ to $\left.9^{\circ} 54^{\prime} \mathrm{N}\right)$ during two Alvin cruises in 1996 and 1997 . All of the vents (whose temperatures ranged from $259^{\circ}$ to $375^{\circ} \mathrm{C}$ ) emitted long wavelength light that corresponded to thermal radiation from a black body at the same temperature. OPUS also detected light at shorter wavelengths $(400-700 \mathrm{~nm})$ that was orders of magnitude more than expected for purely thermal radiation (Figure 1.7) [White et al., 1996b].

The OPUS instrument, while providing helpful preliminary information, was not sufficient to properly characterize light at hydrothermal vents for three main reasons. Its primary deficiency was its lack of imaging capability. The size of the emitting area and the precise region within the hydrothermal plume where light was being emitted could not be determined by the OPUS instrument. Secondly, due to the size of the pressure case, only four photodiodes could be placed in each case which restricted simultaneous observations to only four wavelength bands. Finally, OPUS was merely a time-series recorder. While this allowed observation of temporal variations, the inability to integrate incoming light over long exposures limited its sensitivity to low light levels. Given the fact that the light at vents may vary over a large range in wavelength, space, time, and intensity, a more sophisticated instrument was required. This thesis discusses the design and operations of 
such an instrument — ALISS (Ambient Light Imaging and Spectral System) — and its application to characterizing light emission at deep-sea hydrothermal vents.

\subsection{Thesis OUtLine}

Characterizing the light emission and understanding its possible sources is the next step in understanding the phenomenon of vent light. This will help us to better understand the physical processes occurring at hydrothermal vents, and the environment in which deep-sea biological communities live.

In Chapter 2, I discuss a number of mechanisms that may cause light emission at deep-sea vents. Thermal radiation was the first proposed source of vent light, and it appears to be the most dominant. The physics behind thermal (black body) radiation, unlike most of the other mechanisms, is well understood, and thus, the expected emission can be determined with relative ease. Non-thermal sources, such as sono-, crystallo-, tribo-, and chemiluminescence are not well characterized, but may contribute to the light observed at hydrothermal vents, especially in the visible region of the spectrum. Other sources, such as Cerenkov radiation due to radioactive decay of elements such as ${ }^{40} \mathrm{~K}$ and

${ }^{222} \mathrm{Rn}$, are well characterized, but the emission levels appear to be too low to significantly contribute to the light seen at vents.

In Chapter 3, I describe the ALISS instrument in detail — from the basics of CCD technology, which has greatly improved low-light imaging, to the specifics of the ALISS system and how it obtains the temporal, spatial and spectral information needed to characterize vent light. This chapter also includes a description of the standard image processing techniques used to analyze the ALISS data.

Chapter 4 discusses the physical properties of vent fluids and ambient seawater. These include the temperature and chemistry of vent fluids, the attenuation and scattering of 
light due to seawater in vent environments, and the emissivity of vent fluids. While attempts have been made to characterize attenuation and emissivity in the vent environment, much work remains to be done. Thus, this chapter looks at the theory behind these properties, and discusses how they may be measured on future deployments.

The results of the first two ALISS deployments (in the $9-10^{\circ} \mathrm{N}$ area of the East Pacific Rise, and the Main Endeavour Field on the Juan de Fuca Ridge) are described in Chapter 5. This chapter discusses how emission varies due to vent type, temperature, and chemistry. The photon flux is characterized such that inferences can be made about the sources of vent light.

Chapter 6 summarizes the effectiveness and results of the ALISS camera system. This chapter also discusses how the surrounding biological communities may be affected by light emission from hydrothermal vents. Finally, a series of future deployments and experiments are proposed to further characterize vent light and its possible sources. 


\section{Figure Captrons}

Figure 1.1 World map showing the locations of mid-ocean ridges and subduction zones on the seafloor. Three hydrothermal vent sites - the Main Endeavour Field on the Juan de Fuca Ridge (MEF), the Venture Hydrothermal Field on the East Pacific Rise $\left(9^{\circ} \mathrm{N}\right)$, and the TAG Hydrothermal Field on the Mid-Atlantic Ridge (TAG) - are indicated by shaded circles.

Figure 1.2 Three manifestations of high-temperature, hydrothermal venting:

A) Black smoker chimney $-\sim 350^{\circ} \mathrm{C}$ fluid exits a central chimney conduit and mixes with entrained ambient seawater leading to mineral precipitation; B) Flange pool $-\sim 350^{\circ} \mathrm{C}$ fluid is trapped beneath a sulfide flange forming a relatively stable pool;

C) Beehive or "diffuser" — hydrothermal fluid less than $350^{\circ} \mathrm{C}$ percolates through a matrix of sulfide and exits as clear (i.e., non-precipitating) fluid.

Figure 1.3 A) Vent shrimp Rimicaris exoculata. Lateral view (a) and oblique dorsal view (b) showing location of the photoreceptor organ (stippled area) (c). B) Rhodopsin absorbance curve normalized and compared to frog rhodopsin (dashed line). [FromVan Dover et al., 1989]

Figure 1.4 First image of vent glow taken with a charge-coupled device (CCD) camera at a vent on the Endeavour Segment of the Juan de Fuca Ridge during an Alvin dive cruise in 1988 (Alvin dive 2074). [FromVan Dover et al., 1994a] 
Figure 1.5 A) Observed OPUS counts at a vent in the 9-10 $\mathrm{N}$ EPR area compared to expected counts from a $350^{\circ} \mathrm{C}$ black body source (with an area-emissivity product assumed to be one). Plotted points have a bandwidth of $100 \mathrm{~nm}$ and $25 \%$ error bars. B) Time series of OPUS counts in the $800 \mathrm{~nm}$ channel observed $10 \mathrm{~cm}$ above a vent at Snake Pit on the MAR. [Van Dover et al., 1996]

Figure 1.6 Second generation OPUS instrument - (clockwise from left) front end containing four photodiodes, circuit board, filter assembly.

Figure 1.7 OPUS data from three vents in the 9-10 $\mathrm{N}$ EPR area compared to radiation from $250^{\circ}, 300^{\circ}$, and $350^{\circ} \mathrm{C}$ black body sources. $\mathrm{P}$ vent and $\mathrm{L}$ vent have a temperature of $\sim 375^{\circ} \mathrm{C}$, AdV 4-9 vent has a temperature of $\sim 260^{\circ} \mathrm{C}$. 


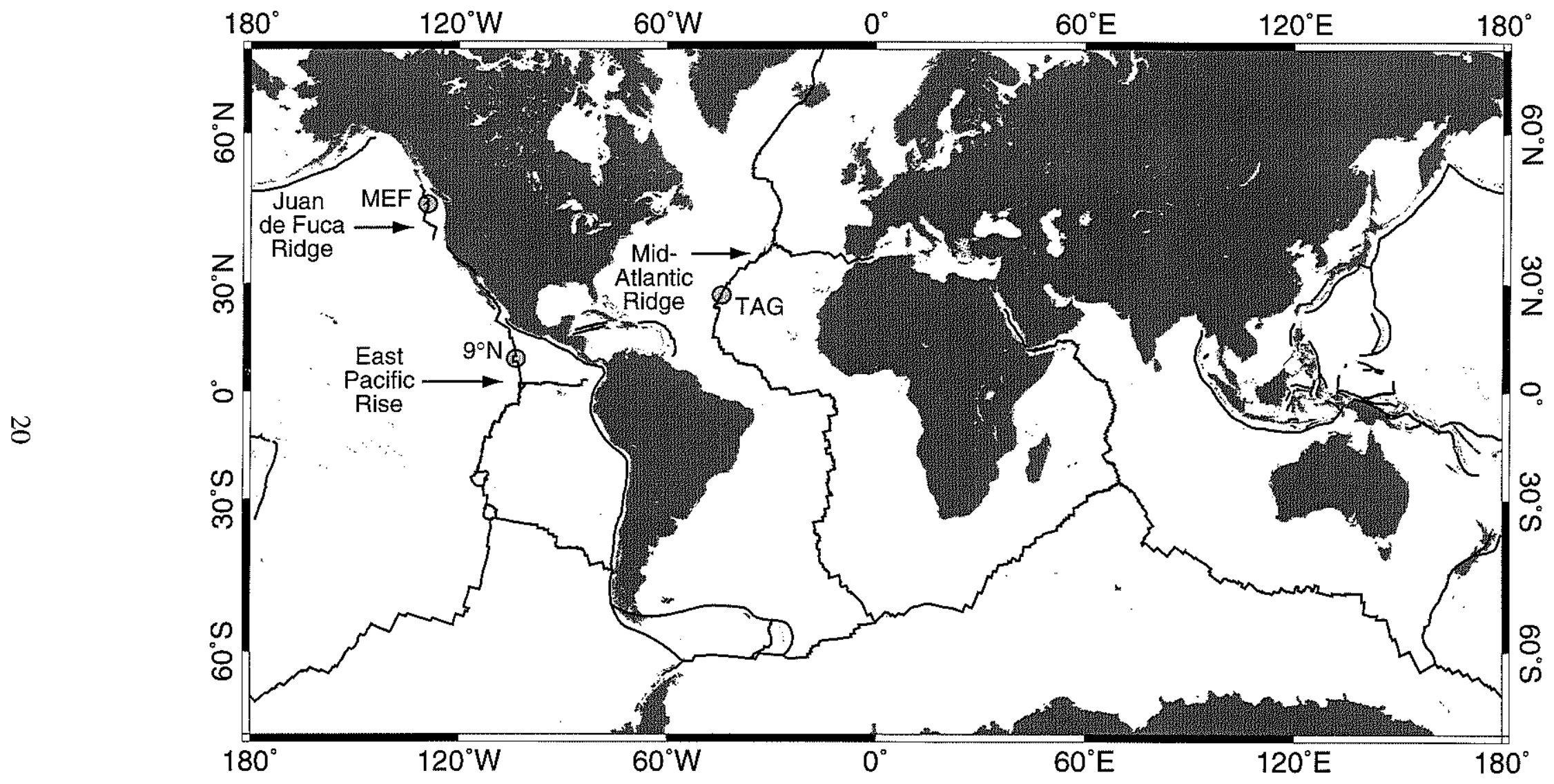

Figure 1.1 
A

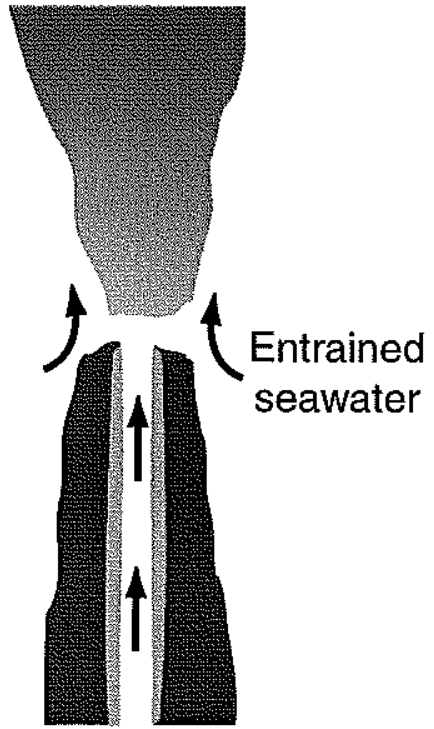

Black Smoker
B

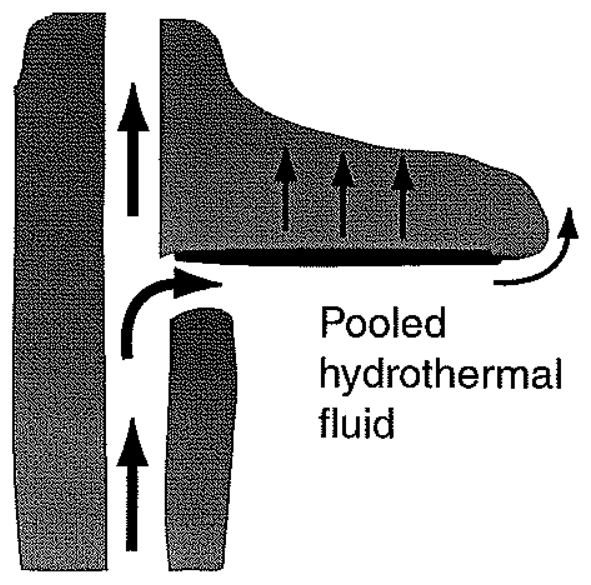

Flange Pool

C

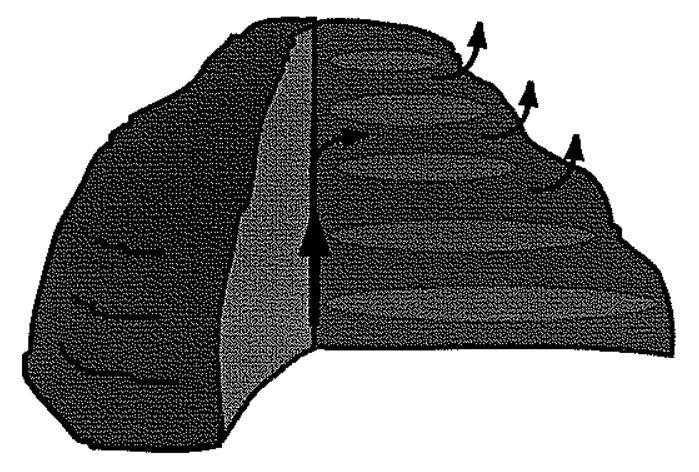

Beehive
("diffuser")

Figure 1.2 
A

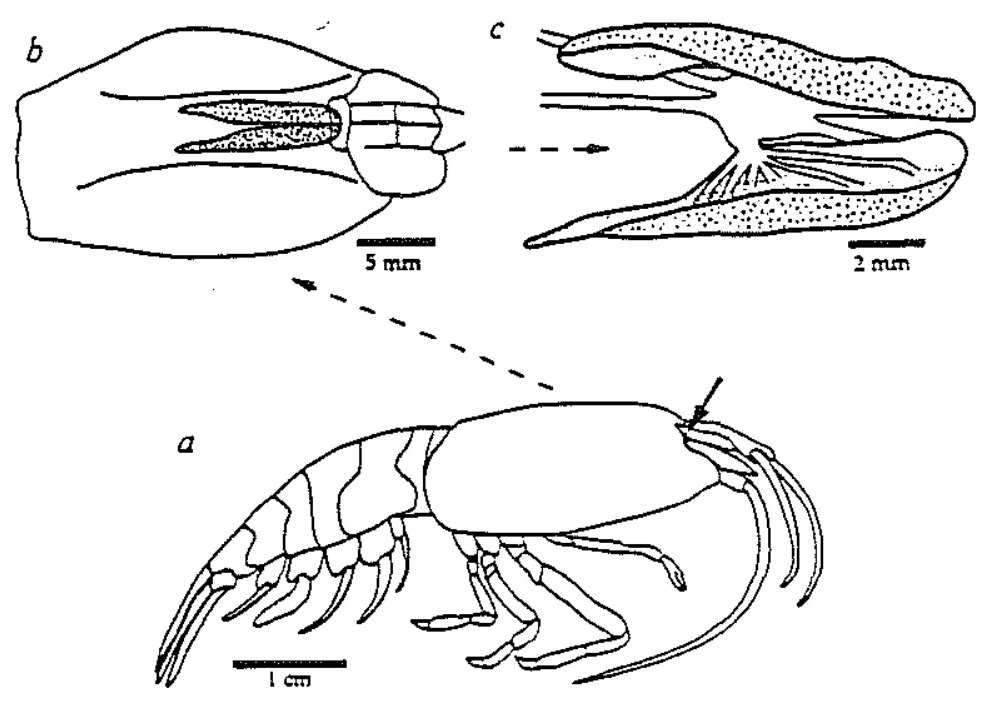

B

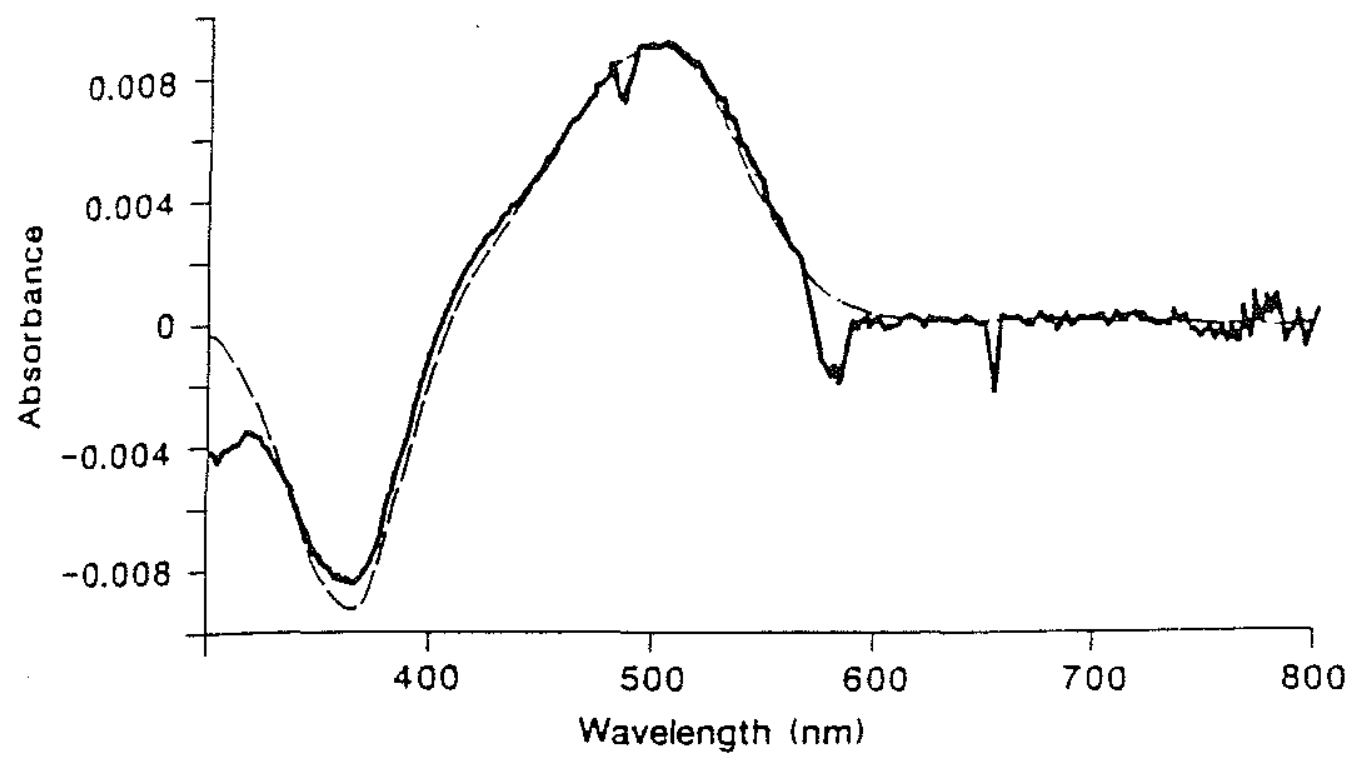

Figure 1.3 


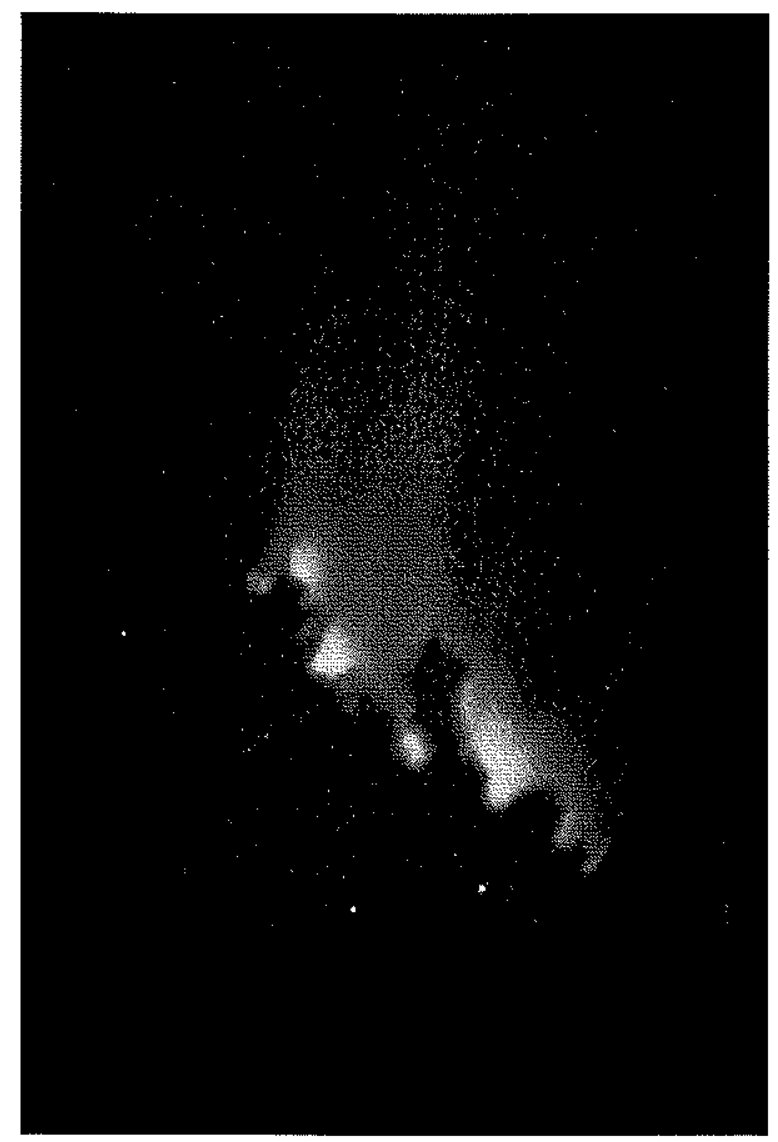

Figure 1.4 

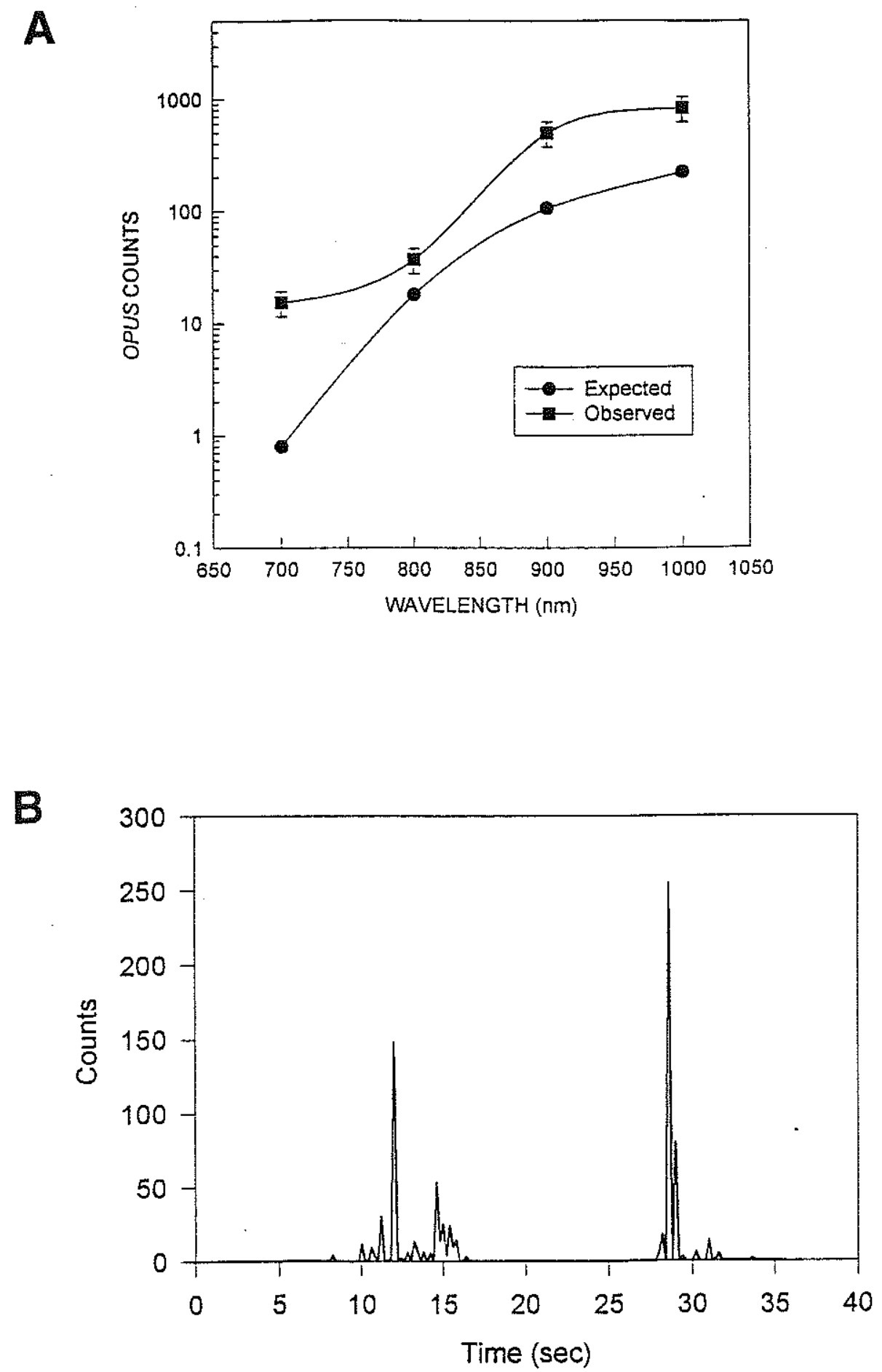

Figure 1.5 


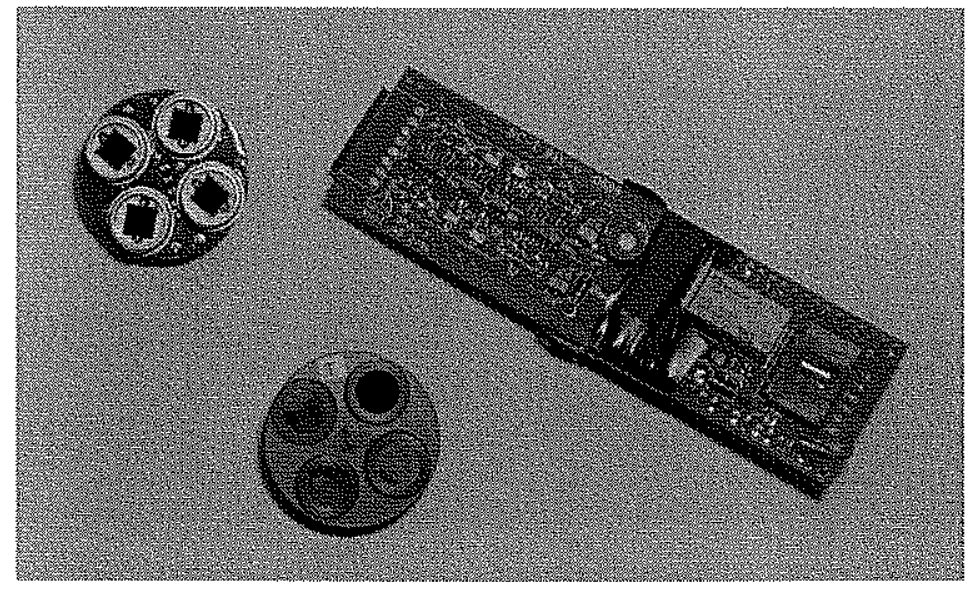

Figure 1.6 


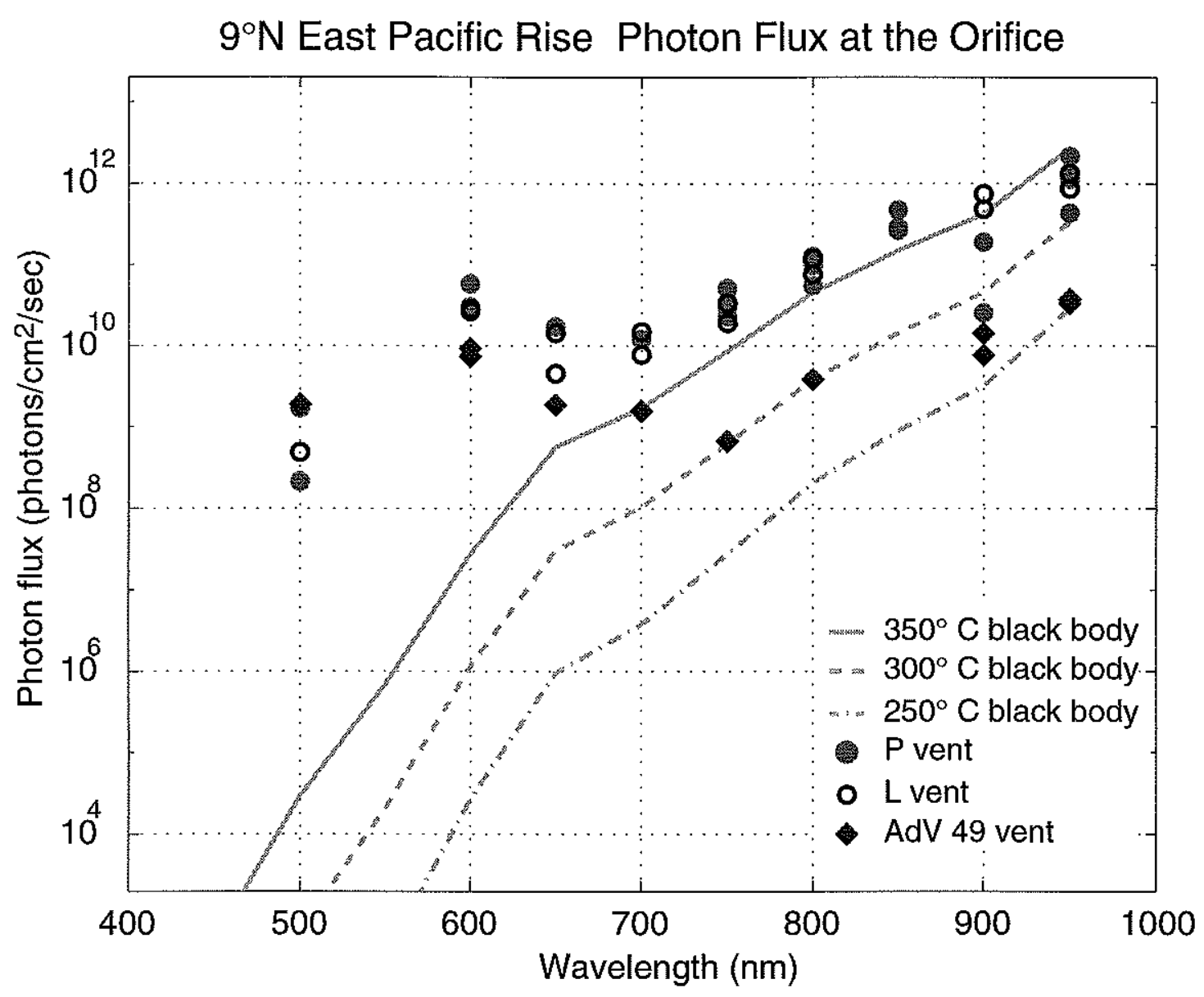

Figure 1.7 


\section{Chapter 2 - Possible Sources of Vent Light}

There are a number of mechanisms that could contribute to light emission at deepsea hydrothermal vents. These mechanisms are related to the various properties of and conditions existing at vents (i.e., temperature, chemistry, turbulent flow, mixing, boiling, precipitation, etc.). Some mechanisms are well understood and their light emission is well characterized, both in terms of spectral distribution and absolute flux. However, some of the mechanisms are not well understood. In these cases, one can only try to understand the conditions required for light emission, general spectral distribution, and possibly upper bounds on photon flux to determine how likely they are to contribute to vent light.

\subsection{Thermal Radiation}

The most plausible source of light at high-temperature hydrothermal vents is thermal (black body) radiation. An ideal black body is perfectly opaque and non-reflecting. Hence, it is a perfect absorber and a perfect emitter. In reality, most objects are not perfect absorbers or emitters. They are sometimes referred to as "gray bodies." The ratio of energy emitted by an object to the energy emitted by an ideal black body at the same temperature is called emissivity, $\varepsilon$. Thus, an ideal black body has an emissivity of one, and a gray body has an emissivity less than one. (The concept of emissivity and how to measure it will be discussed further in Section 4.3).

Thermal radiation - the electromagnetic energy emitted from the surface of a heated body - is solely dependent on temperature. This can be seen in Boltzmann's Law which states that the total energy emitted by a black body per square meter per second $\left(W_{B}\right)$ increases as the fourth power of the absolute temperature $(T)$ :

$$
W_{B}=\sigma \cdot T^{4}
$$


where $\sigma$ is the Stefan-Boltzmann constant $\left(5.670 \times 10^{-8} \mathrm{~J} /\left(\mathrm{m}^{2} \cdot \mathrm{s} \cdot \mathrm{K}^{4}\right)\right)$. Thus, the only requirement for thermal radiation is heat. The intensity and distribution of the radiation can be described by Planck's Law and Wien's Displacement Law, respectively. The variation in intensity across the spectrum is described by Planck's Law. For hot water of temperature, $T$, radiating into seawater, the number of photons per second per square meter per unit solid angle between wavelengths $\lambda$ and $\lambda+d \lambda$ is given by

$$
I(\lambda, T)=A \cdot \mathcal{E} \cdot \frac{c n^{2}}{\lambda^{4}}\left[e^{h c / \lambda k T}-1\right]^{-1} d \lambda
$$

where $A$ is the emitting area, $\varepsilon$ is the emissivity of the vent fluid, $c$ is the speed of light in vacuo $\left(\sim 3.0 \times 10^{8} \mathrm{~m} / \mathrm{s}\right), n$ is the index of refraction of seawater (1.33), $h$ is Planck's constant $\left(6.6256 \times 10^{-34} \mathrm{~J} \cdot \mathrm{s}\right)$, and $k$ is the Boltzmann constant $\left(1.3805 \times 10^{-23} \mathrm{~J} / \mathrm{K}\right)$. Wien's Law states that the wavelength at which the radiation is maximum $\left(\lambda_{\max }\right)$ is inversely proportional to the absolute temperature $(T)$ :

$$
\lambda_{\max }=\frac{2.897 \times 10^{-3} \mathrm{~m} \cdot K}{T}
$$

For $350^{\circ} \mathrm{C}(623.15 \mathrm{~K})$ black smoker fluid, the radiation peaks in the infrared at $\sim 4600 \mathrm{~nm}$, but with a long tail that extends into the visible region $(350-750 \mathrm{~nm})$ - the amount of energy radiated in the far red/near infrared $(750-1150 \mathrm{~nm})$ is $4 \times 10^{13}$ times that radiated into the visible $(350-750 \mathrm{~nm})$. As the temperature increases, the peak wavelength decreases. Thus, the peak emission of the sun, with a temperature of $\sim 6000 \mathrm{~K}$, is at $\sim 500 \mathrm{~nm}$.

As stated above, the thermal radiation emitted by a body is dependent on temperature alone. With temperatures sometimes as high as $400^{\circ} \mathrm{C}$ [e.g., Von Damm et al., 1995a], the laws of physics dictate that high-temperature vent fluid must emit thermal radiation. The light emission from a $350^{\circ} \mathrm{C}$ black body with unit area and an emissivity of one is shown in Figure 2.1. If thermal radiation is a component of vent light, then one 
would expect to see this trend of increasing intensity with increasing wavelength in the data. In fact, early data obtained with the OPUS instrument are consistent with the black body model at long wavelengths $(>700 \mathrm{~nm})$.

There are, however, three problems in predicting the thermal radiation at hydrothermal vents: (1) determining the actual temperature, (2) determining the emissivity, and (3) determining the actual emitting area.

\section{1) Determining actual temperature}

The temperature of a vent can be measured by the Alvin temperature probe. It is common practice for the tip of the probe to be inserted deep into the vent orifice in order to find the highest temperature of the vent fluid (i.e., the temperature of the end-member fluid prior to mixing with seawater). While this temperature is important for understanding vent processes, it is not necessarily the temperature of the fluid being imaged by ALISS. As the high-temperature hydrothermal fluid exits the orifice, it mixes with entrained, ambient seawater $\left(\sim 2^{\circ} \mathrm{C}\right)$. This causes a rapid decrease in temperature above the orifice. Indeed, the fluid can drop to $100^{\circ} \mathrm{C}$ in as little as 10 to $20 \mathrm{~cm}$. The temperature gradient across the plume (i.e., horizontally) is even sharper $-2^{\circ} \mathrm{C}$ to $~ 300^{\circ} \mathrm{C}$ within a centimeter.

When measuring the temperature of black smoker vents imaged by ALISS, an effort was made to measure the temperature of the fluid at the orifice and at 5 to $10 \mathrm{~cm}$ increments above the orifice. These numbers are useful, but still not exact as the $\sim 0.5 \mathrm{~cm}$ wide tip of the temperature probe disrupts the temperature of the flow it is measuring. However, the temperature of flange pools and beehive structures can be measured rather accurately, as flange pools are relatively stable and both are backed by hot rock of the same temperature. 


\section{2) Determining emissivity}

The second problem in predicting the black body radiation at hydrothermal vents is the value of emissivity. Emissivity can be a difficult concept to understand with respect to a transparent, or semi-transparent medium. As noted above, emissivity is related to absorbance, but matter such as water and glass has little to no absorbance (at least in the visible region of the spectrum). Hydrothermal plumes are not transparent due to the existence of mineral particles in the plume. Plumes emit light; therefore they must have a non-zero value of emissivity. Since the vent environment cannot be easily reconstructed in the lab, a method of determining emissivity in situ must be developed. A discussion of how emissivity will be measured on future ALISS deployments is in Section 4.3.

\section{3) Determining actual emitting area}

Finally, it is important to know the emitting area of the vents - that is, the area of the plume at a given temperature. Unlike the OPUS instrument, the ALISS camera actually images the vents, which enables us to determine the area of the vent that is glowing. However, because of the temperature gradients, it is difficult to determine what area of the plume is radiating at, say, $330^{\circ} \mathrm{C}$. Thus, we can only estimate the emitting area. For black smokers, we assume the emitting area to be on the order of a few square centimeters. Since flanges have a uniform temperature, we can calculate exactly from the ALISS images the size of the area we are observing.

\subsection{Cerenkov Radiation}

Cerenkov radiation is the light generated by a charged particle traveling through a medium with a velocity greater than that of light in the medium. This phenomenon is common in nuclear reactors, where high-energy beta particles (electrons) from the 
radioactive core create a bluish glow in the surrounding cooling water. While Cerenkov radiation was discovered by Mallet in 1926 and subsequently studied by Cerenkov (for whom it was named), Frank and Tamm were the first to develop the theoretical explanation based on classical physics [ Collins and Reiling, 1938; Belcher, 1953; Jelley, 1958, and references therein].

When a charged particle moves through a medium, the atoms of the medium behave like dipoles causing local polarization. At low velocities (much less than the speed of light), the polarization is symmetric (Figure 2.2A) and there is no resultant field. At high velocities, the polarization is asymmetric (Figure 2.2B) and a brief electromagnetic pulse is released. In general, the generated waves interfere destructively. However, if the particle velocity is greater than the speed of light in the medium, the waves will constructively interfere and generate a cone of light that is symmetric about the particle's path (Figure 2.3). The cone angle, $\theta$, is given by the Cerenkov relation:

$$
\cos \theta=\frac{1}{\beta \cdot n}
$$

where $n$ is the index of refraction of the medium, and $\beta$ is the ratio of the particle velocity to the speed of light in vacuo $\left(c \approx 3.0 \times 10^{8} \mathrm{~m} / \mathrm{s}\right)$. This is analogous to a shock wave generated by an object traveling faster than the speed of sound with $\beta \cdot n$ equivalent to the Mach number. In an isotropic medium containing radioactive isotopes, the electrons are emitted uniformly in all direction and, hence, the angular distribution of emission is uniform.

The speed of light in vacuo is a threshold that cannot be surpassed by any particle. However, in a medium with an index of refraction of $n$, the speed of light is reduced to $c / n$, a value that can be surpassed by a high-energy particle. The index of refraction of water is 1.33. Thus, the speed of light in water is $\sim 2.25 \times 10^{8} \mathrm{~m} / \mathrm{s}$. This corresponds to an electron energy greater than $0.26 \mathrm{MeV}$ or an alpha particle energy greater than $2 \mathrm{GeV}$. The presence 
of the radioactive isotope ${ }^{40} \mathrm{~K}$ in the ocean causes light emission throughout the ocean - a background emission that must be accounted for when studying bioluminescence [e.g., Bradner et al., 1987]. Since ${ }^{40} \mathrm{~K}$ and other radioactive isotopes are present in vent fluids in higher concentrations than in normal sea water [e.g., Kadko and Moore, 1988], Cerenkov radiation must be considered as a possible source of vent light.

The number of photons emitted into a half-space per square centimeter per second is given by the following formula [Belcher, 1953; Geelhood, 1982; Roberts, 1979]:

$$
\text { PhotonFlux }=A \cdot \frac{2 \pi}{137}\left(\frac{1}{\lambda_{2}}-\frac{1}{\lambda_{1}}\right)\left(1-\frac{1}{\beta^{2} n^{2}}\right)\left(\frac{1}{100}\right) \cdot l \cdot \frac{L}{4}
$$

where $A$ is the activity of the radioactive isotope (in disintegrations $/ \mathrm{cm}^{3} / \mathrm{sec}$ ), $\lambda_{I}-\lambda_{2}$ is the spectral range (in meters), $\beta$ is the ratio of the particle velocity to the speed of light in vacuo, $n$ is the index of refraction of seawater, $l$ is the path length of the particle (in centimeters), and $L$ is the attenuation length (in centimeters). Since the flux is inversely proportional to wavelength, the highest emission is at the shorter wavelengths (i.e., in the visible region rather than the infrared).

Vent fluids contain a number of radioactive isotopes that could contribute to Cerenkov radiation (e.g., ${ }^{40} \mathrm{~K},{ }^{210} \mathrm{~Pb},{ }^{210} \mathrm{Po},{ }^{222} \mathrm{Rn}$, and ${ }^{226} \mathrm{Ra}$ ). While both ${ }^{40} \mathrm{~K}$ and ${ }^{210} \mathrm{~Pb}$ are beta emitters, only the electron from ${ }^{40} \mathrm{~K}$ decay has an energy greater than the Cerenkov threshold of $0.26 \mathrm{MeV}$. The concentration of potassium in vent fluids can be two to three times higher than in seawater $(\sim 9.8 \mathrm{mmol} / \mathrm{kg})$ [e.g., Butterfield et al., 1994]. Assuming a potassium concentration of $24.5 \mathrm{mmol} / \mathrm{kg}$ (from Lobo Flange on the Juan de Fuca Ridge) [Butterfield et al., 1994] and a fractional abundance of $1.17 \times 10^{-4}$, the photon flux at 450 $\mathrm{nm}$ is $\sim 5$ photons $/ \mathrm{cm}^{2} / \mathrm{sec} / \mathrm{str}$ (Figure 2.4 ) - steradian (str) is a unit of solid angle into which light is emitted from a point source. Many other species are alpha emitters; however, their energies are below the Cerenkov threshold and their path length orders of 
magnitude less than the path length of electrons. Although ${ }^{222} \mathrm{Rn}$ is an alpha emitter, a 3.28 $\mathrm{MeV}$ electron is emitted further down ${ }^{222} \mathrm{Rn}$ 's decay path as ${ }^{214} \mathrm{Bi}$ decays to ${ }^{214} \mathrm{Po}$ (Figure 2.5). This electron is much more energetic than that emitted from ${ }^{40} \mathrm{~K}$ and produces an order of magnitude more radiation ( 50 photons $/ \mathrm{cm}^{2} / \mathrm{sec} / \mathrm{str}$ ). This appears to be the highest emitter in vent fluids; however, it is still a low level of light and most likely does not significantly contribute to vent light.

\subsection{SONOLUMINESCENCE}

Sonoluminescence (SL) is the emission of light during cavitation of a liquid. Cavitation is the generation of cavities (or bubbles) in a liquid due to variations in pressure - caused by water impinging on water [Anbar, 1968], the movement of a propeller [e.g., Walton and Reynolds, 1984], acoustic cavitation [e.g., Sehgal et al., 1979; Crum and Reynolds, 1985], or flow through a venturi tube [e.g., Weninger et al., 1999], to name a few mechanisms. However, not all of these forms of cavitation lead to light emission. While this phenomenon has been known for $\sim 60$ years, physicists are still trying to understand the exact processes at work in generating light [Walton and Reynolds, 1984, and references therein].

Most of the research on sonoluminescence has involved acoustic cavitation, as it provides an easy means of generating cavitation in the lab. Cavities are formed when the negative pressure associated with the expansion cycle of the sound wave overcomes the tensile strength of the liquid. In a pure liquid, the tensile strength is very high (on the order of 1000 bar for water at room temperature). However, the tensile strength is reduced orders of magnitude (to $\sim 1$ bar) by the existence of "cavitation nuclei" — gas trapped in the crevices of small particles, or microbubbles remaining from previous cavitation events [Walton and Reynolds, 1984; Suslick, 1990]. Small bubbles generated in the liquid grow 
with each pressure cycle until they reach a critical size (on the order of 100 to $200 \mu \mathrm{m}$ in diameter). At this size, a bubble is able to efficiently absorb energy such that, during the subsequent pressure cycle, it expands rapidly to the point where it becomes unstable and collapses (Figure 2.6). During implosion, the concentration of energy generates a hot spot with a temperature on the order of thousands of degrees Kelvin [Flint and Suslick, 1991a]. Photons emitted by the collapsing bubble can have an energy twelve orders of magnitude higher than the energy density of the sound field, and the light emitted from the collapsing bubble has a pulse width on the order of 100 picoseconds $\left(10^{-12}\right.$ seconds) or less [Barber and Putterman, 1991].

Generally in nature, one observes multiple bubble sonoluminescence (MBSL) where the light emission is due to the collapse of a number of bubbles. Recently, experimenters have been able to generate single bubble sonoluminescence (SBSL) where a single bubble is trapped in the antinode of a standing wave [Gaitan et al., 1991]. This bubble regularly oscillates and emits light with clock-like precision [Barber and Putterman, 1991]. Through SBSL, researchers have been better able to understand the mechanism of sonoluminescence. However, studies show distinct differences in the spectral emission from SBSL and MBSL, which may represent fundamentally different processes of light emission. MBSL spectra are characterized by emission lines from hydroxyl $(\mathrm{OH}-310$ $\mathrm{nm}$ ) and alkali metals in the solution (e.g., sodium - $589 \mathrm{~nm}$ ) [ Sehgal et al., 1979; Flint and Suslick, 1991b; Becker et al., 1992; Didenko and Pugach, 1994]. In contrast, SBSL spectra do not show emission lines, and instead appear to correlate well to black body emission from a source 25,000 K or higher [Hiller et al., 1992]. Crum [1994] proposed that, in contrast to symmetrical collapse during SBSL, bubbles in MBSL collapse asymmetrically (due to interference by adjacent bubbles) allowing liquid to be deposited within the bubble and subsequently heated during implosion. Thus, light emission from 
SBSL and MBSL is dominated by the characteristics of the gas and liquid, respectively. Yasui [1999] suggests that the lack of emission lines in SBSL is due to quenching by higher temperatures and pressures inside the bubble $-10,000-50,000 \mathrm{~K}$ and $10^{9}-10^{10} \mathrm{~Pa}$ for SBSL in contrast to $3000-5000 \mathrm{~K}$ and $10^{7}-10^{8} \mathrm{~Pa}$ for MBSL.

Sonoluminescence is a likely contributor to light emission at deep-sea vents. Vents in the Main Endeavour Field on the Juan de Fuca Ridge (2200 m depth) are known to undergo subcritical phase separation (boiling) at or just below the seafloor [Butterfield et $a l ., 1994]$. However, boiling, in general, does not cause luminescence [Walton and Reynolds, 1984]. This may be explained by Harvey's [1939] observation that sonoluminescence disappears when ambient temperatures reach $85-90^{\circ} \mathrm{C}$. Additionally, this may be due to the fact that bubbles created during boiling continue to expand and explode, rather than implode. However, boiling in the subsurface may create the cavitation nuclei necessary for cavitation to occur at the vent orifice from a venturi effect.

The venturi effect is the change in velocity and pressure of a fluid as it flows through a duct of varying cross-sectional area. The relationship between velocity and area is given by

$$
A_{1} \cdot V_{1}=A_{2} \cdot V_{2}
$$

where $A_{1}$ and $V_{1}$, and $A_{2}$ and $V_{2}$ are the cross-sectional area and velocity, respectively, at two points in the duct. The velocity of the flow is also related to pressure as shown by Bernoulli's equation:

$$
p+\frac{1}{2} \rho \cdot V^{2}=\text { const }
$$

where $p$ is the pressure, $\rho$ is the density, and $V$ is the velocity of the fluid. Thus, flow that travels through a constriction experiences higher velocities and lower pressures within the constriction (Figure 2.7A). 
In a high-temperature vent, flow is focused through a narrow orifice before being released at the seafloor (Figure 2.7B). Thus, cavities may form during the low pressure phase in the constriction, and implode during the return to higher pressure beyond the constriction. The pressure difference is calculated from:

$$
p_{2}-p_{1}=\frac{1}{2} \rho \cdot V^{2} \cdot\left(\frac{A_{2}}{A_{1}}-1\right)
$$

where the subscript 1 indicates conditions within the constriction, and subscript 2 indicates conditions after expansion. The density $(\rho)$ of sea water is $\sim 1025 \mathrm{~kg} / \mathrm{m}^{3}$, and exit velocities are on the order of 1-2 m/s [Ginster et al., 1994]. Therefore, a pressure drop of 1 bar requires a radius ratio $\left(r_{2} / r_{1}\right)$ of 7 to 14 . This suggests that cavities formed within the chimney structure will implode some distance above the orifice where the flow has greatly expanded (Figure 2.7B).

Recent work by Reynolds [2000] describes a newly identified type of SL referred to as "Vapor Bubble Luminescence" (VBL). This type of luminescence is associated with the condensing of macroscopic vapor bubbles in water - produced by injecting steam (via a cappuccino maker) into water. Given its similarity to hydrothermal vents, VBL must be considered a possible source of vent light.

No experimental work yet exists that provides a reasonable estimate of sonoluminescent emission at deep-sea hydrothermal vents. The SL spectra of converging flow has been shown to mimic the spectra of sonically induced MBSL, although at lower intensities [Weninger et al., 1999]. It is also possible that the extreme temperatures and pressures present at deep-sea vents may affect the SL photon flux. Dan et al. [1999] show how emission varies with ambient pressure $\left(\mathrm{P}_{0}\right)$ between 0.7 and $1.0 \mathrm{~atm}$. If the acoustic driving pressure $\left(\mathrm{P}_{\mathrm{a}}\right)$ is held constant, photon flux decreases with increasing pressure. However, if the $\mathrm{P}_{\mathrm{o}} / \mathrm{P}_{\mathrm{a}}$ ratio is held constant, photon flux increases with increasing pressure. 
Jarman [1960] and Weninger et al. [1999] observe a decrease in emission with increasing ambient temperature - a drop by a factor of $\sim 3$ from $25^{\circ}$ to $40^{\circ} \mathrm{C}$ down to undetectable levels above $40^{\circ} \mathrm{C}$. Neither of the above experiments deal with temperatures and pressures as high as those seen on the seafloor, nor do they utilize sea water. Thus it is difficult to relate these findings to fluxes at deep-sea vents.

Two different types of observations could be made to detect sonoluminescence or cavitation at vents. One way to detect SL at vents is to look for distinct emission lines in the spectrum. Sodium is prevalent in seawater and hydrothermal fluid, and its spectrum is characterized by a strong doublet emission at $\sim 589 \mathrm{~nm}$ [Becker et al., 1992] (Figure 2.8). Thus, by looking narrowly at the $589 \mathrm{~nm}$ region, we might expect to find a sonoluminescent signal. It might also be worthwhile to look for the $310 \mathrm{~nm}$ emission from $\mathrm{OH}^{-}$. However, Taylor and Jarman [1970] show that the $\mathrm{OH}^{-}$band is quenched by the presence of salts (e.g., sodium chloride). Since light at these wavelengths may be due to mechanisms other than SL, a second method is to use a hydrophone to detect sound emission resulting from cavitation. Reynolds et al. [1982] observed that SL from acoustic cavitation was not observed until an audible signal indicating cavitation could be heard. Hydrophones deployed at $21^{\circ} \mathrm{N}$ on the East Pacific Rise [Riedesel et al., 1982] and at the Ashes Vent Field on the Juan de Fuca Ridge [Little et al., 1990] have detected lowfrequency noise $(\sim 0.1-40 \mathrm{~Hz})$ in the vicinity of hydrothermal vents. The source of the low-frequency noise could not be clearly ascertained in either location, but appears to be associated with black smoker vents. An attempt was made during a 1996 Alvin cruise to the East Pacific Rise to detect cavitation with a hydrophone. Unfortunately, the hydophone did not function and no data were collected. This method should be attempted again on future cruises. 


\subsection{Tribo- and Crystalloluminescence}

Light emission can be associated with both the crystallization of minerals and the fracturing of minerals - crystalloluminescence (XTL) and triboluminescence (TL), respectively. The name triboluminescence comes from the Greek tribo meaning "to rub". Triboluminescence is light emission due to application of mechanical energy to a solid (i.e., by crushing, thermal shock, cracking, etc.). Observations of triboluminescence of sugar date as far back as the 1600's [Walton, 1977, and references therein]. Although unfamiliar with the name, many people today are aware that biting down on a wintergreen LifeSaver ${ }^{(0)}$ will produce a triboluminescent spark.

Crystalloluminescence is sometimes classified as a sub-type of TL. XTL occurs during rapid crystallization of a mineral (i.e., $\mathrm{NaCl}$ ) from an aqueous solution [Gibbon et al., 1988]. Garten and Head [1963; 1970] suggest that light emission is derived from the energy released during a phase change. That is, when minerals are forming in a solution, the nuclei gather in amorphous aggregates. When the aggregate reaches a critical size, it "clicks" into crystalline form and energy is released as light.

A number of minerals are known to be TL and XTL active [Walton, 1977; Reynolds, 1995, and sources therein]. Sphalerite $(\mathrm{ZnS})$ is a TL-active mineral that is prevalent at many hydrothermal vents - both within the chimney structure and as precipitates in the plume. Sphalerite precipitates from hydrothermal fluid as it cools to temperatures ranging from $180^{\circ} \mathrm{C}$ to $255^{\circ} \mathrm{C}$ [Haymon, 1983; Baron, 1998]. It is also found in the middle and outer portions of the chimney walls (both on the East Pacific Rise and the Juan de Fuca Ridge [Haymon, 1983; Tivey and McDuff, 1990; Koski et al., 1994]. Triboluminescent studies of sphalerite by Nelson [1926] showed luminescence in the yellow region of the spectrum $(\sim 550-600 \mathrm{~nm})$. 
More recently, actual chimney samples were analyzed for TL emission. Zink [1981] and Zink and Chandra [1982] showed that the TL and XTL spectra of a crystal closely resemble each other and the photoluminescent (PL) spectrum. Therefore, by analyzing the PL spectrum of a mineral excited by a laser, one can estimate the TL and XTL spectra. Chimney samples from two vents (M vent \& $\mathrm{L}$ vent) from $9^{\circ} \mathrm{N}$ on the East Pacific Rise were analyzed by this method [Radziminski et al., 1997]. Samples were excited by an $\mathrm{Ar}^{+}$laser $(351.1 \mathrm{~nm})$ and observed in the $360-750 \mathrm{~nm}$ range. The $\mathrm{M}$ vent sample was predominately anhydrite $\left(\mathrm{CaSO}_{4}\right)$, and showed no PL emission. The $\mathrm{L}$ vent sample was divided into three sections: (1) innermost surface — predominately chalcopyrite $\left(\mathrm{CuFeS}_{2}\right)$; (2) middle - chalcopyrite and anhydrite; and (3) outermost surface - pyrite $\left(\mathrm{FeS}_{2}\right)$, anhydrite, and very little sphalerite. Little to no light emission was seen from the outer and middle sections. The inner section, however, showed a strong emission in the $400-500 \mathrm{~nm}$ range (Figure 2.9). This suggests that chalcopyrite may be a strong TL and/or XTL emitter in the blue region of the spectrum.

Little work has been done to determine the absolute intensities of TL emission from crystals. Beese and Zink [1984] looked at TL emission from a number of different minerals (e.g., sucrose and $\mathrm{ZnSO}_{4} \cdot 7 \mathrm{H}_{2} \mathrm{O}$ ). They found absolute fluxes ranging from $6.2 \times 10^{10}$ photons $/ \mathrm{mol}$ (for $\mathrm{MgSO}_{4} \cdot 6 \mathrm{H}_{2} \mathrm{O}$ ) to $5.0 \times 10^{16}$ photons $/ \mathrm{mol}$ (for cholesteryl salicylate). Caminite $\left(\mathrm{MgSO}_{4} \cdot 1 / 3 \mathrm{Mg}(\mathrm{OH})_{2} \cdot 1 / 3 \mathrm{H}_{2} \mathrm{O}\right)$ is precipitated when seawater is heated to temperatures of $\sim 250-350^{\circ} \mathrm{C}$ [Bischoff and Seyfried, 1978]. It is found in hydrothermal chimneys intimately associated with anhydrite. Assuming that all of the $\mathrm{Mg}$ in a $10 \mathrm{~cm}^{3}$ area is precipitated as caminite, and that the XTL flux is $6.2 \times 10^{10}$ photons $/ \mathrm{mol}$, the light detected at the ALISS camera in the visible region would barely be above that predicted for thermal radiation. 
TL and XTL active minerals are present at hydrothermal vents [e.g., Haymon and Kastner, 1981; Tivey and McDuff, 1990; Baron, 1998] and are known emit light in the visible region of the spectrum [e.g., Nelson, 1926; Radziminski et al., 1997]. Although absolute intensity is not yet characterized for many of these minerals, triboluminescence and crystalloluminescence must be considered possible components of vent light.

\subsection{Chemiluminescence}

Chemiluminescence (CL) is the conversion of chemical energy to light. During some chemical reactions, it is possible for an atom or molecule to become excited. That is, one of its electrons is raised to a higher energy level. On return to the ground state, the drop in energy of the electron leads to the emission of a photon whose frequency (or, reciprocally, wavelength) is dependent on the energy. For example,

$$
A^{*} \rightarrow A+h v
$$

where $A^{*}$ is the excited atom, and $A$ is the ground-state atom. The photon is described by its frequency, $v$, and Planck's constant $\left(h=6.626 \times 10^{-34} \mathrm{~J} / \mathrm{Hz}\right)$. Chemiluminescence is not an uncommon phenomenon and, in fact, is responsible for the light emission by biological organisms (bioluminescence).

Given the chemical processes occurring at vents, it seems quite likely that chemiluminescence contributes in some way to vent light. Unfortunately, at present, there is not a large amount of literature regarding inorganic $\mathrm{CL}$, and the light emitted is not well characterized spectrally or in terms of absolute fluxes. Also, the extreme temperatures and pressures involved at hydrothermal vents cannot easily be reproduced in the laboratory, making it more difficult to understand how CL may be manifested at hydrothermal vents. 
Recently, Tapley et al. [1999] documented chemiluminescence during sulfide oxidation in seawater (this was previously suggested by J. W. Hastings in LITE Workshop Report [1993]). Using a photomultiplier tube sensitive to the wavelengths $380-620 \mathrm{~nm}$, they observed greater luminescence with higher sulfide concentrations (Figure 2.10) [Tapley, 1993]. They also noted decreased emission with the presence of a chelator, which supports the findings that metal catalysis plays a role in sulfide oxidation [e.g., Chen et al., 1972; Vazquez et al., 1989]. Indeed, as noted by Tapley et al. [1999], hydrothermal vent fluids contain significant concentrations of sulfide and undergo rapid mixing with oxygenated seawater entrained in the vent plume. The fluids also contain metals leached from rocks during circulation through oceanic crust. Thus, chemiluminescence is very likely to occur at hydrothermal vents. 


\section{Figure Captions}

Figure 2.1 Photon flux (photons $/ \mathrm{cm}^{2} / \mathrm{sec} / \mathrm{str}$ ) from a $350^{\circ} \mathrm{C}$ ideal black body. Peak emission is at $\sim 4600 \mathrm{~nm}$. The shaded area indicates the visible region of the spectrum. Dashed lines indicate the region of the spectrum analyzed with the ALISS camera system at deep-sea vents.

Figure 2.2 Local polarization of the atoms of a medium through which a charged particle (black dot) is passing with a velocity, (A) $v<<c$, and (B) $v \approx c$, where $c$ is the speed of light in vacuo $\left(\sim 3 \times 10^{8} \mathrm{~m} / \mathrm{s}\right)$. [Modified from Jelley, 1958]

Figure 2.3 Huygen's construction illustrating the coherence of wave-causing Cerenkov radiation. A charged particle traveling faster than the speed of light in the medium will generate waves at arbitrary points $\mathrm{P}_{1}, \mathrm{P}_{2}, \mathrm{P}_{3}$ along path $\mathrm{AB}$. At an angle $\theta$ the waves are coherent and will combine to form a plane wave front BC. [Modified from Jelley, 1958]

Figure 2.4 A) Calculated photon flux (photons $/ \mathrm{cm}^{2} / \mathrm{sec} / \mathrm{str}$ ) due to Cerenkov radiation from ${ }^{40} \mathrm{~K}$ decay in vent fluids at Lobo on the Juan de Fuca ridge (potassium concentration $\approx 24.5 \mathrm{mmol} / \mathrm{kg}$ ). B) Predicted ALISS measurements of the above emission at $50 \mathrm{~cm}$ distance. Each diamond indicates the flux detected through an individual $100 \mathrm{~nm}$ nominal bandwidth filter.

Figure 2.5 The decay path for ${ }^{222} \mathrm{Rn}$. A $3.28 \mathrm{MeV}$ electron is emitted as ${ }^{214} \mathrm{Bi}$ decays to ${ }^{214} \mathrm{Po}$. The rate limiting half-life is that of ${ }^{222} \mathrm{Rn}$ (3.8 days). 
Figure 2.6 Generation of sonoluminescence in a liquid irradiated with ultrasound. The bubble grows during episodes of negative pressure. When it reaches a critical size, the bubble implodes, generating intense heat and pressure (indicated as HOT SPOT on the figure). [From Suslick, 1989]

Figure 2.7 A) Flow through a constriction of cross-sectional area $A_{1}$ causes a decrease in pressure $\left(\mathrm{p}_{1}\right)$ and an increase in velocity $\left(\mathrm{V}_{1}\right)$. As the flow expands to a cross-sectional area of $A_{2}$, the velocity decreases $\left(V_{2}<V_{1}\right)$ and the pressure increases $\left(\mathrm{p}_{2}>\mathrm{p}_{\mathrm{t}}\right)$. B) Flow through a sulfide chimney may mimic this effect by being forced through a narrow orifice and then allowed to expand.

Figure 2.8 Sonoluminescence spectra of (a) unfiltered deep water from the N. Atlantic $\left(24^{\circ} 31^{\prime} \mathrm{N}, 64^{\circ} 01^{\prime} \mathrm{W}\right),(\mathbf{b}) 10.65 \mathrm{~g} / 1 \mathrm{NaCl}$ control, and (c) tap water. The spectral measurements were carried to longer wavelengths, but no emission features were observed. [From Becker et al., 1992]

Figure 2.9 Photoluminescence spectra of different sections of L vent chimney $\left(9^{\circ} \mathrm{N}\right.$ EPR): inner surface - chalcopyrite $\left(\mathrm{CuFeS}_{2}\right)$; center - chalcopyrite and anhydrite $\left(\mathrm{CaSO}_{4}\right)$; outer surface - pyrite $\left(\mathrm{FeS}_{2}\right)$, anhydrite, and very little sphalerite $(\mathrm{ZnS})$. The tail seen at $360 \mathrm{~nm}$ is from the $\mathrm{Ar}^{+}$laser $(351.1 \mathrm{~nm})$ used to excite the sample. The 2 nd harmonic of the laser is seen at $700 \mathrm{~nm}$. The small peak seen at $426 \mathrm{~nm}$ for the center sample is probably a Raman peak - laser light scattered at a different wavelength. (The data have been offset for ease of viewing). [From Radziminski et al., 1997] 
Figure 2.10 Time series of chemiluminescence, measured as counts per second (cps), during sulfide oxidation with varying concentrations of sulfide, with and without chelators. Each point is the average of three determinations; error bars are \pm one standard deviation. Sulfide concentrations: $0 \mu \mathrm{m}-\square, 200$ $\mu \mathrm{m}-\diamond, 500 \mu \mathrm{m}-\boldsymbol{\Delta}$. Chelators were added to $500 \mu \mathrm{m}$ sulfide concentrations: EDTA (ethylenediaminetetraacetic acid) $-\boldsymbol{\nabla}$, DTPA (diethylenetriaminepentaacetic acid) - +. [From Tapley et al., 1999] 


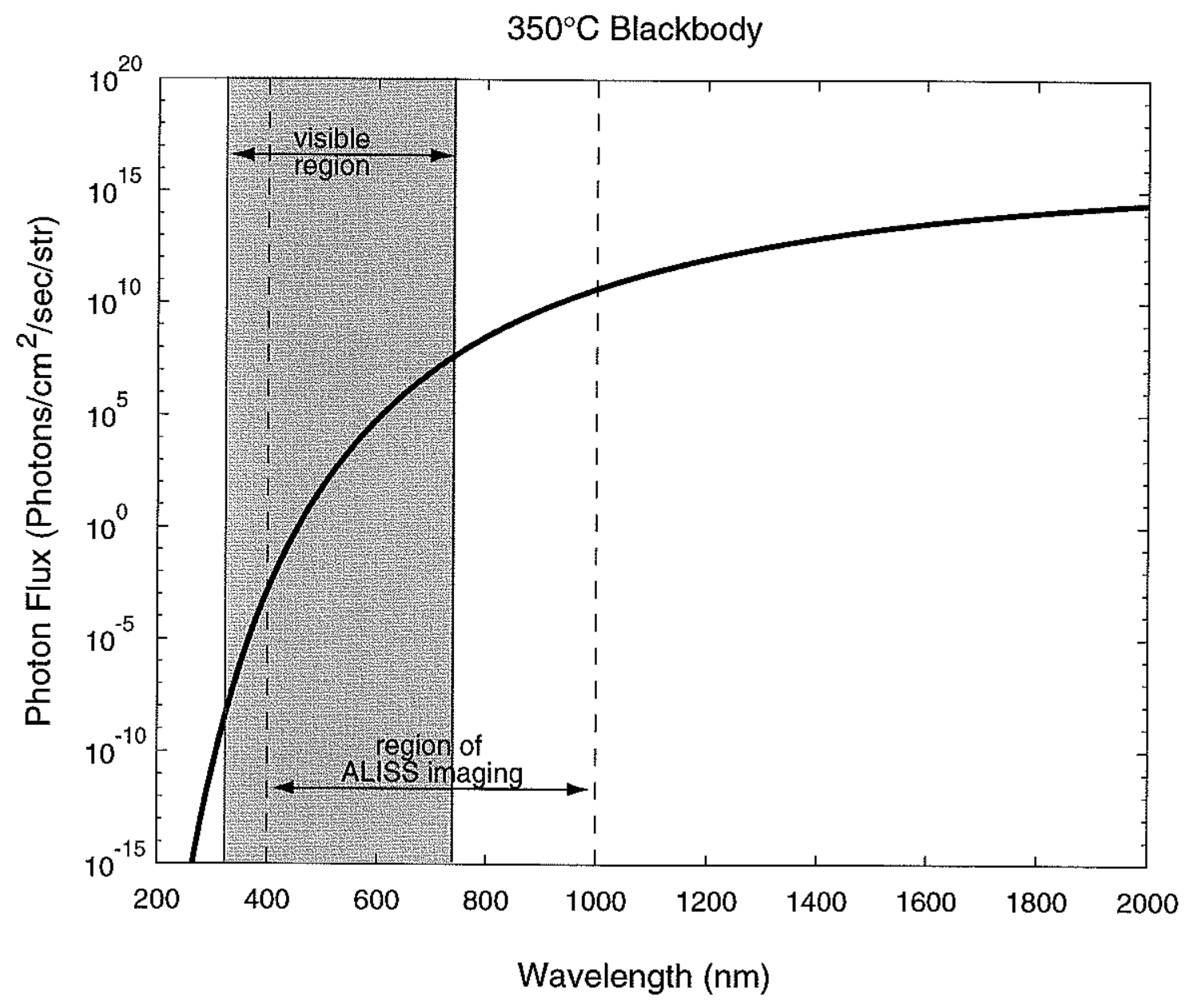

Figure 2.1 


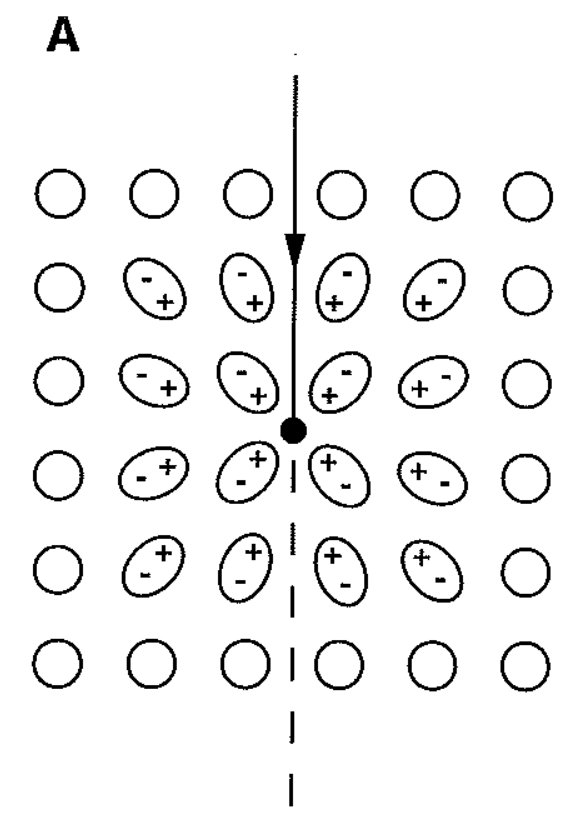

Iow velocity, v $<$ c
B

0001000

$0 \odot \odot \oplus 0$

$0 \odot \odot \oplus 0$

$\circ \odot \odot \oplus 0$

$0 \oplus \odot \oplus 0$

000,000

high velocity, $v \approx c$

Figure 2.2 


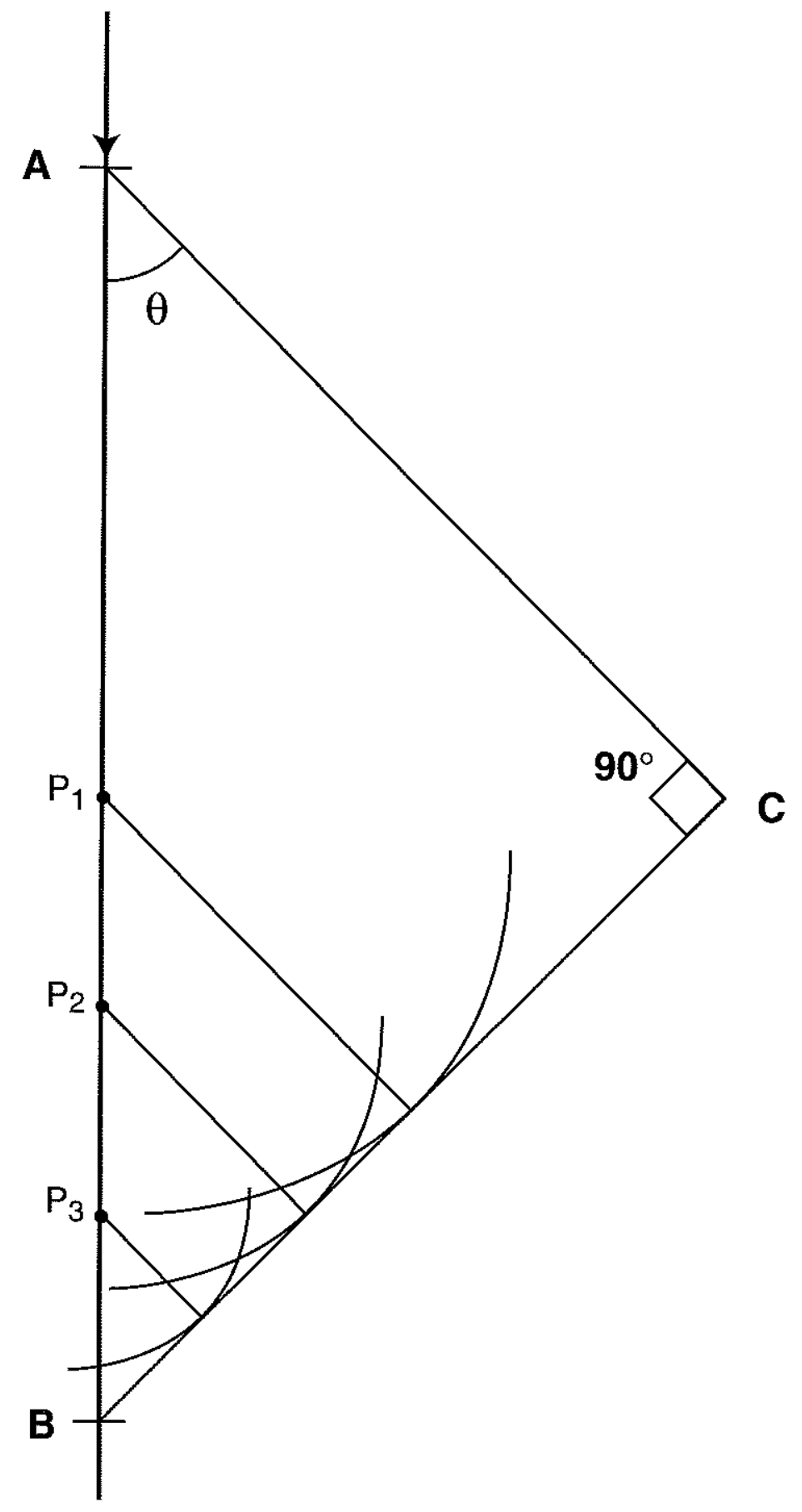

Figure 2.3 

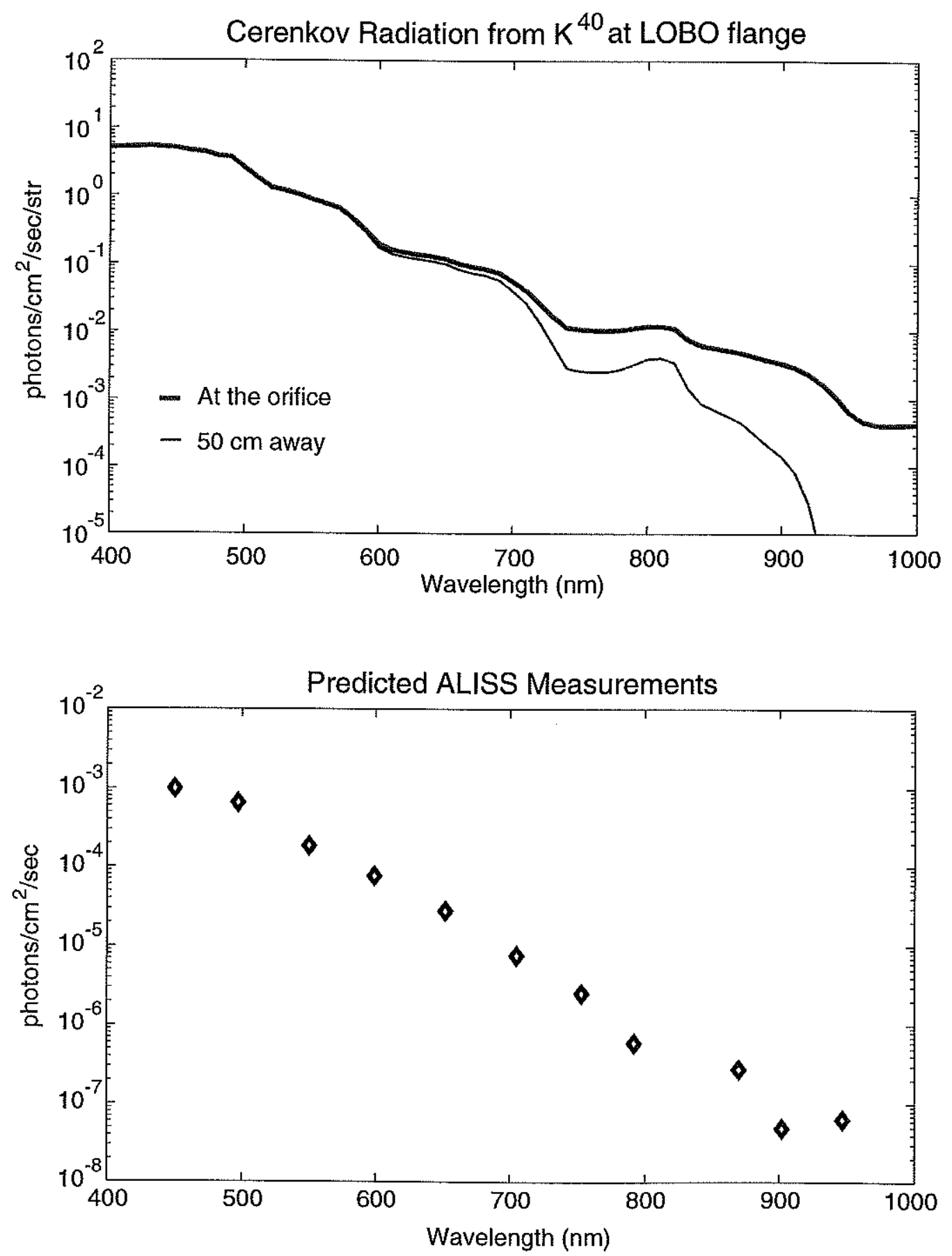

Figure 2.4 


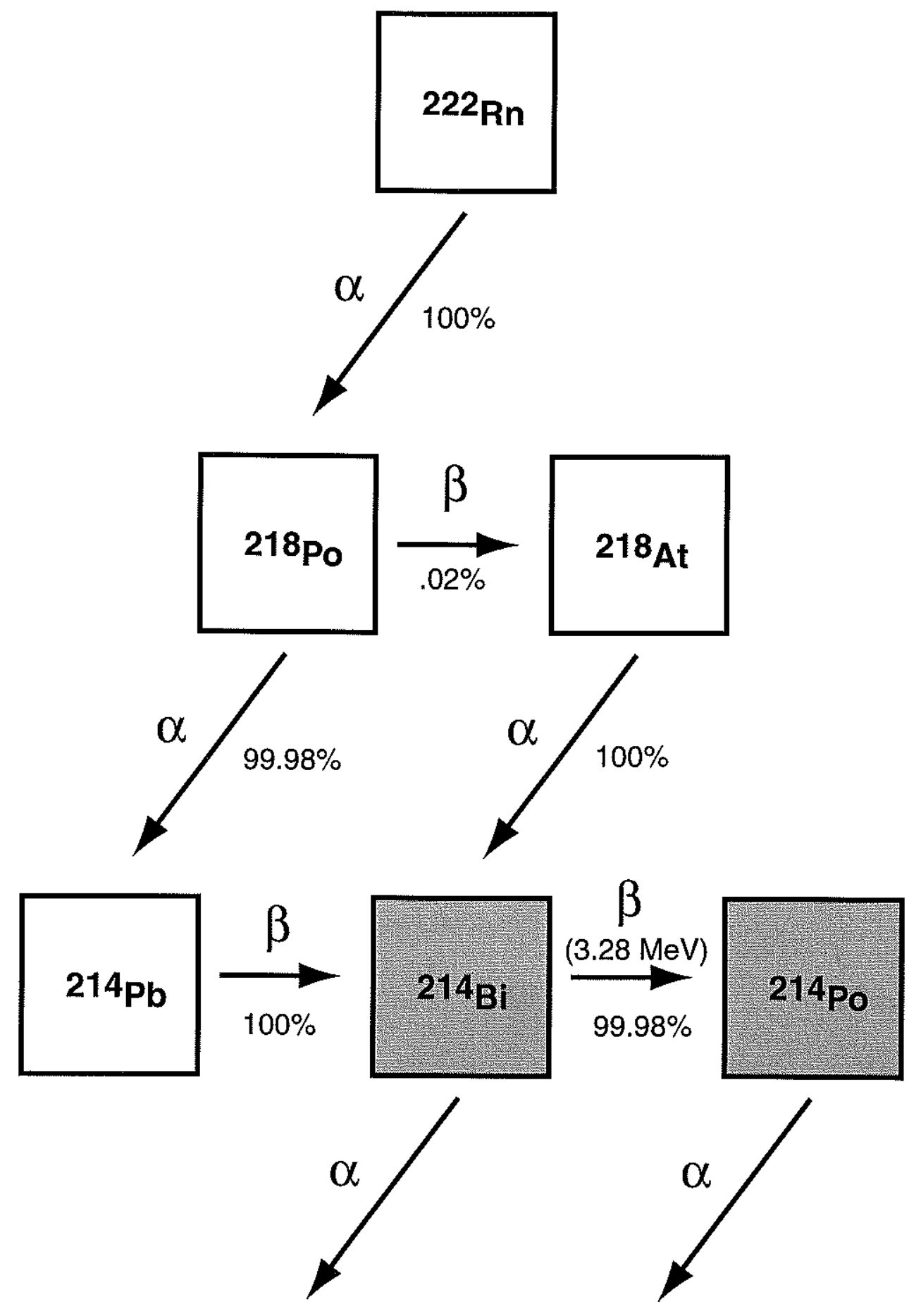

Figure 2.5 


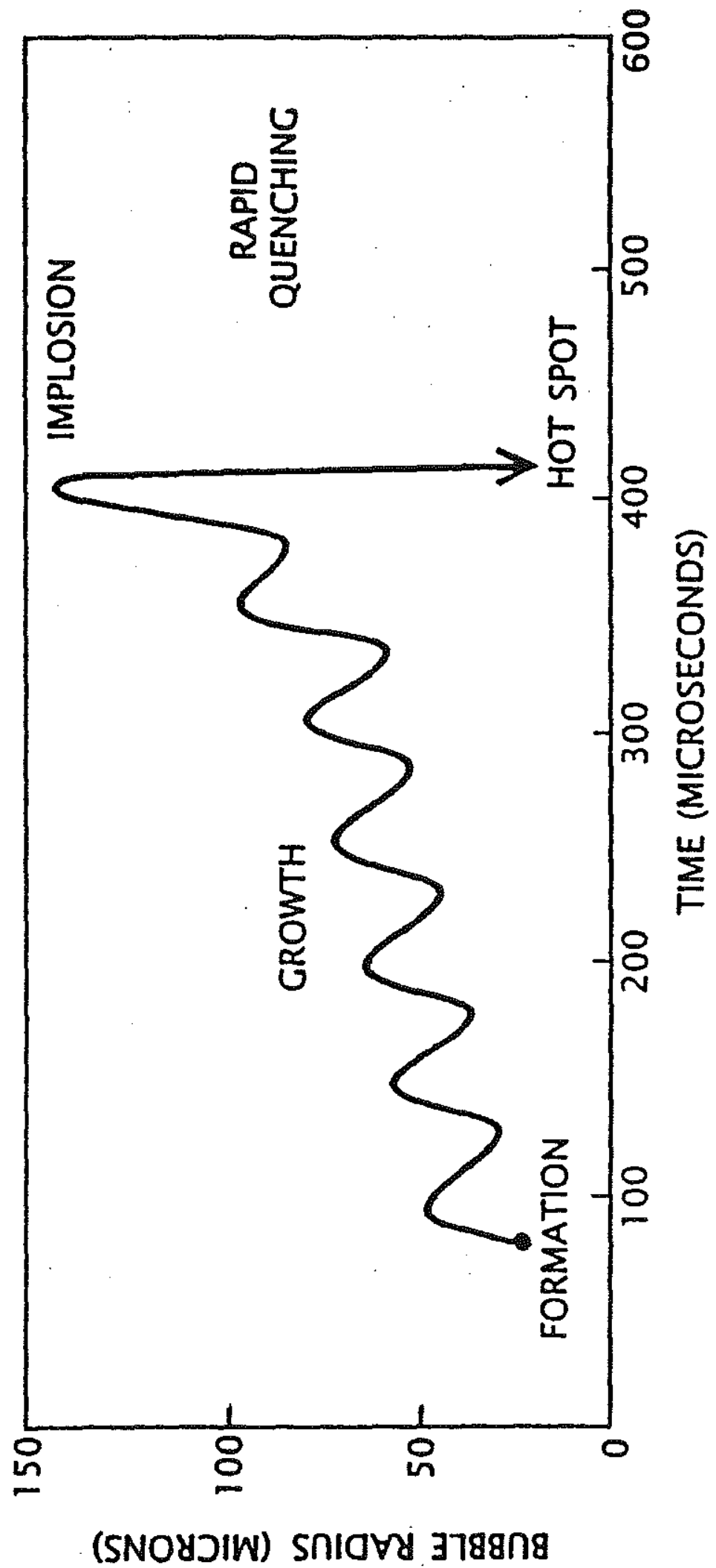

0
0
0
0
0
0 


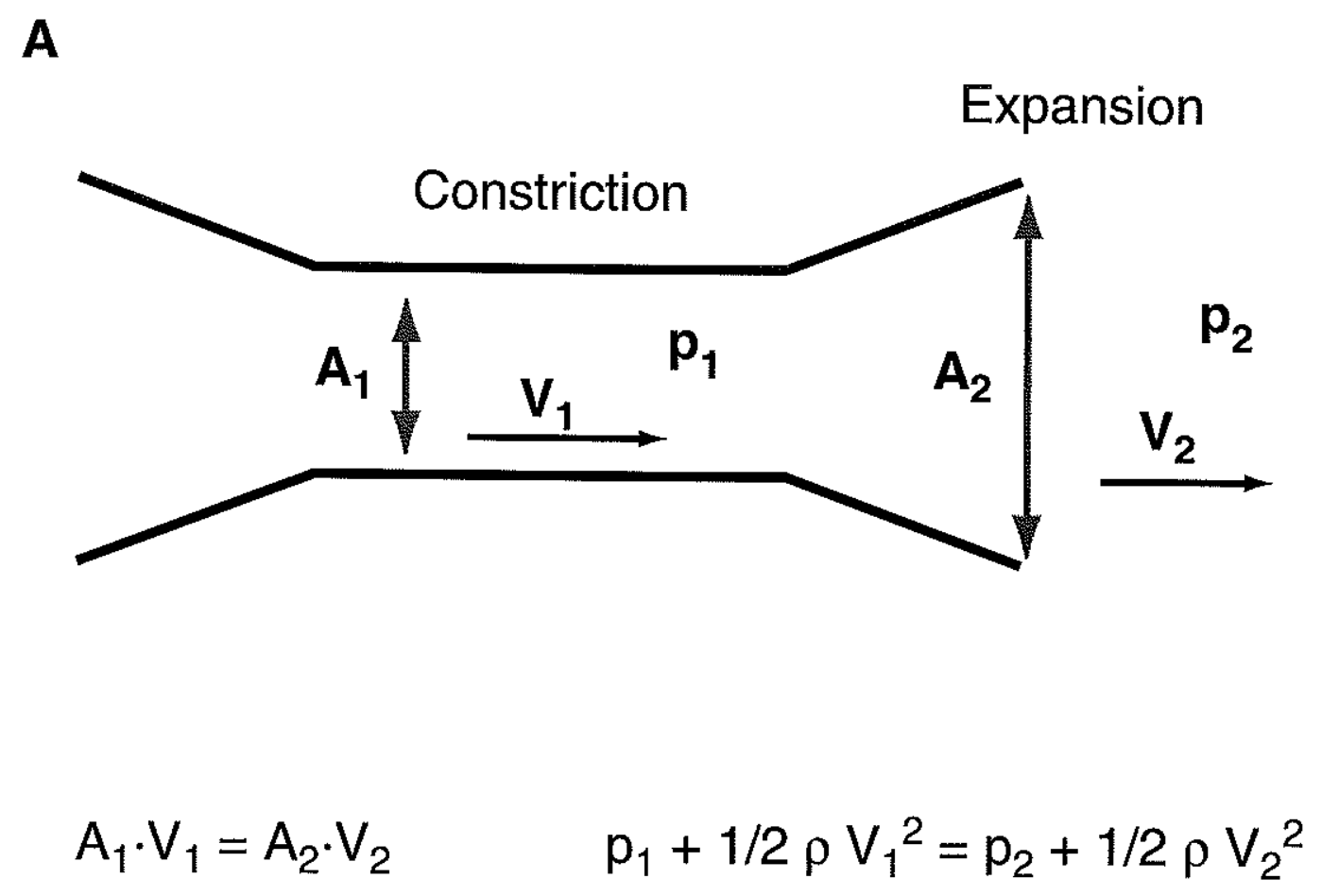

B

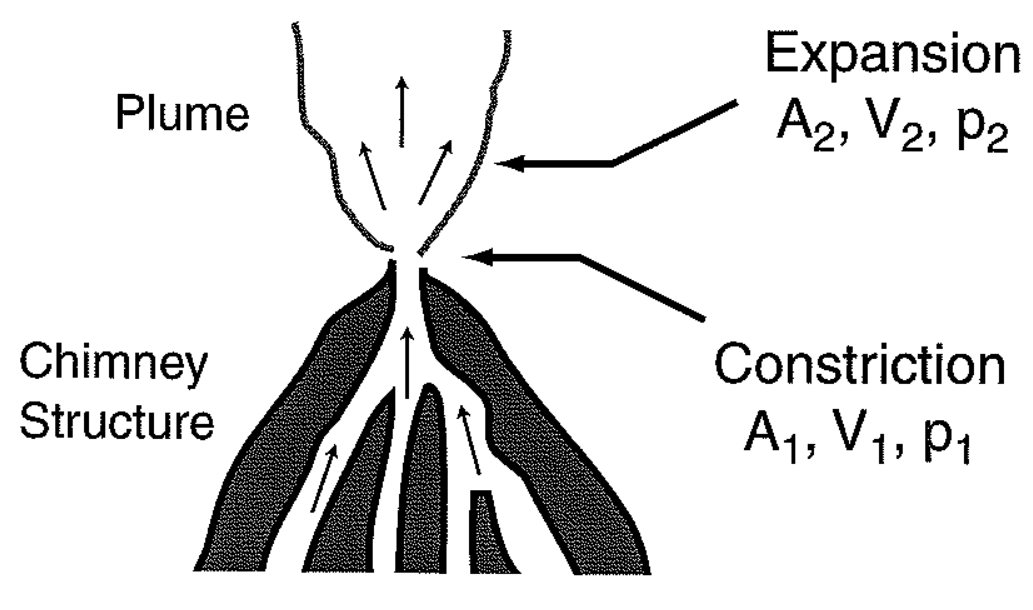

Figure 2.7 


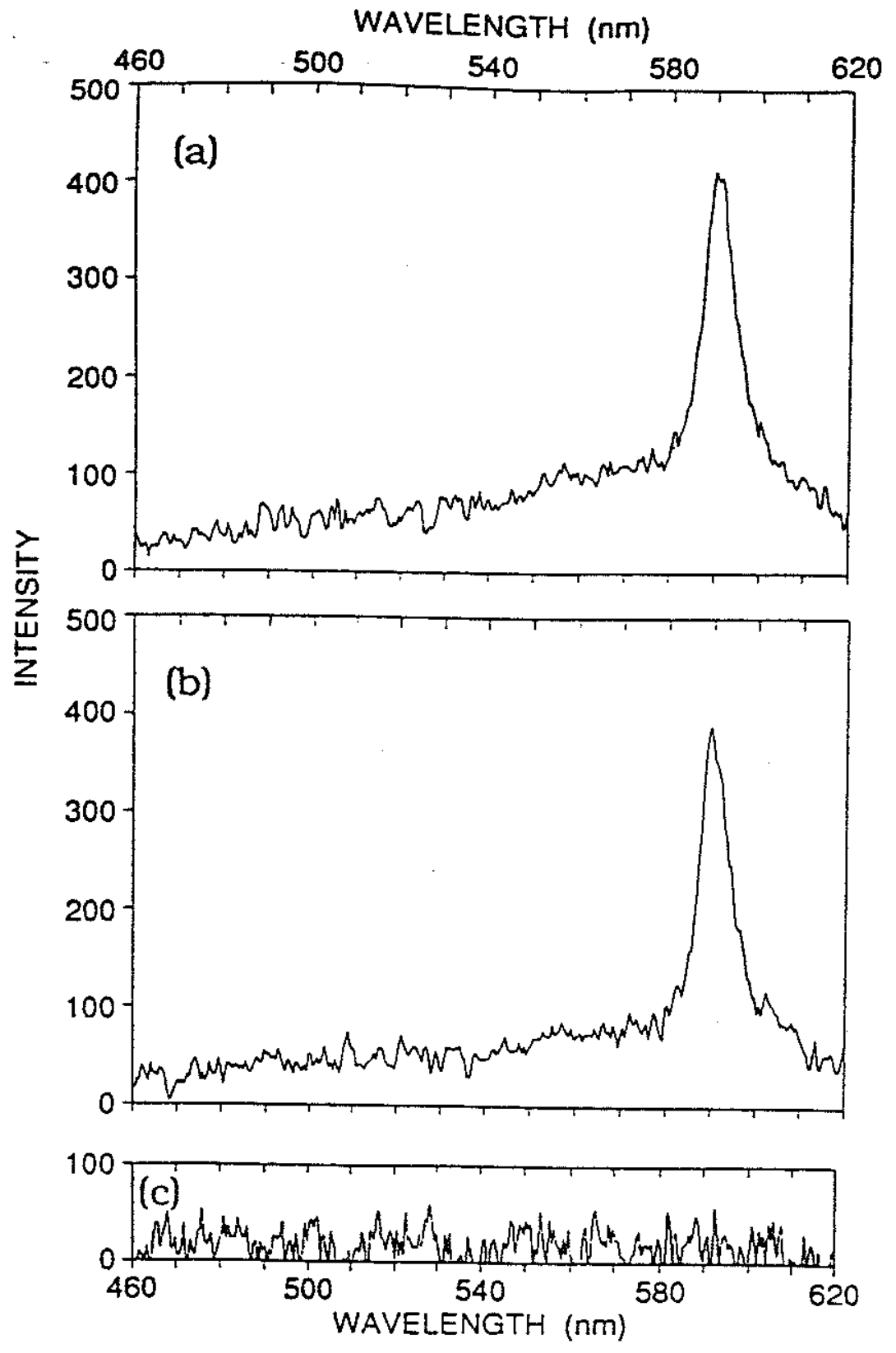

Figure 2.8 
Photoluminescence of $L$ Vent Chimney Sample

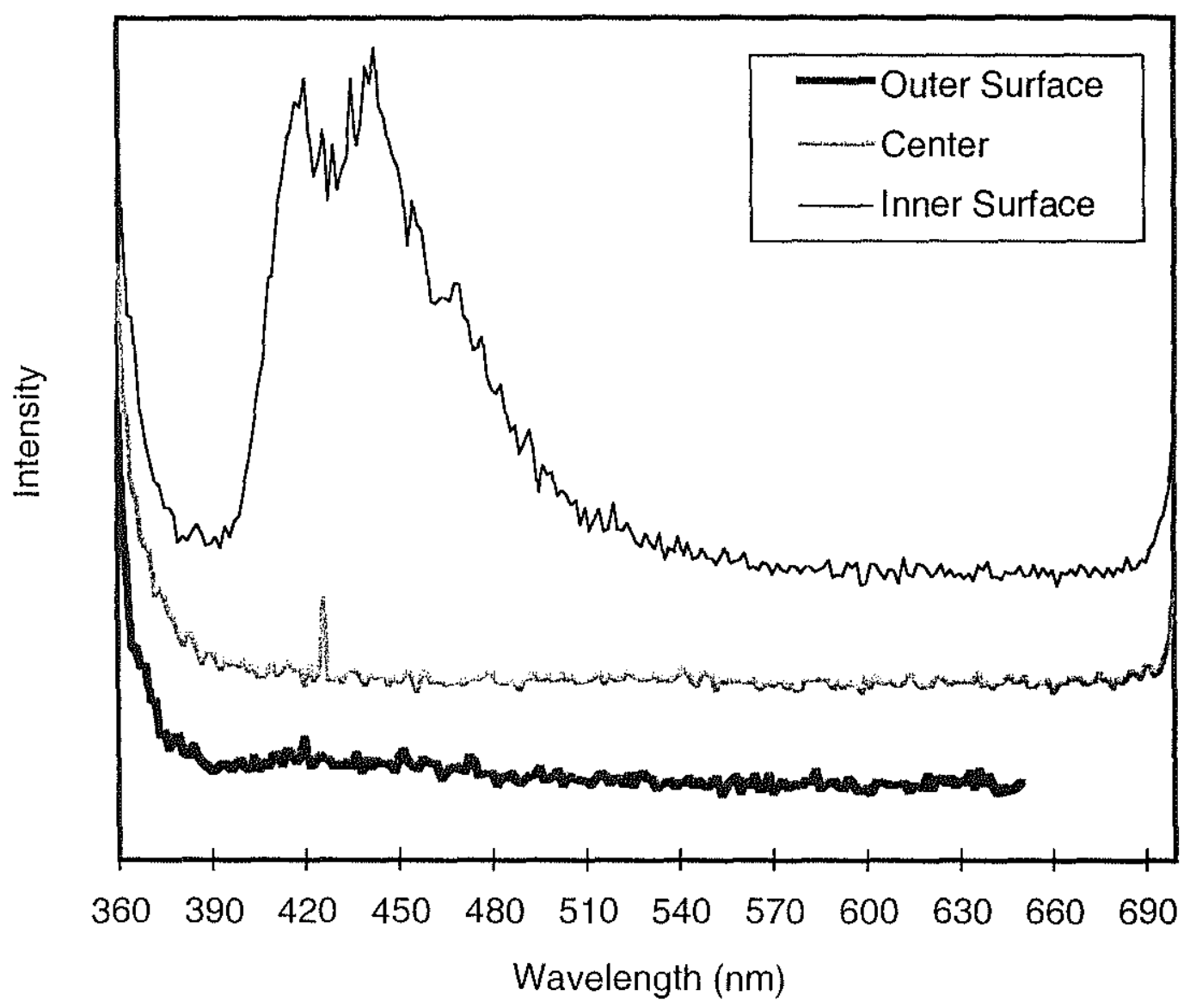

Figure 2.9 


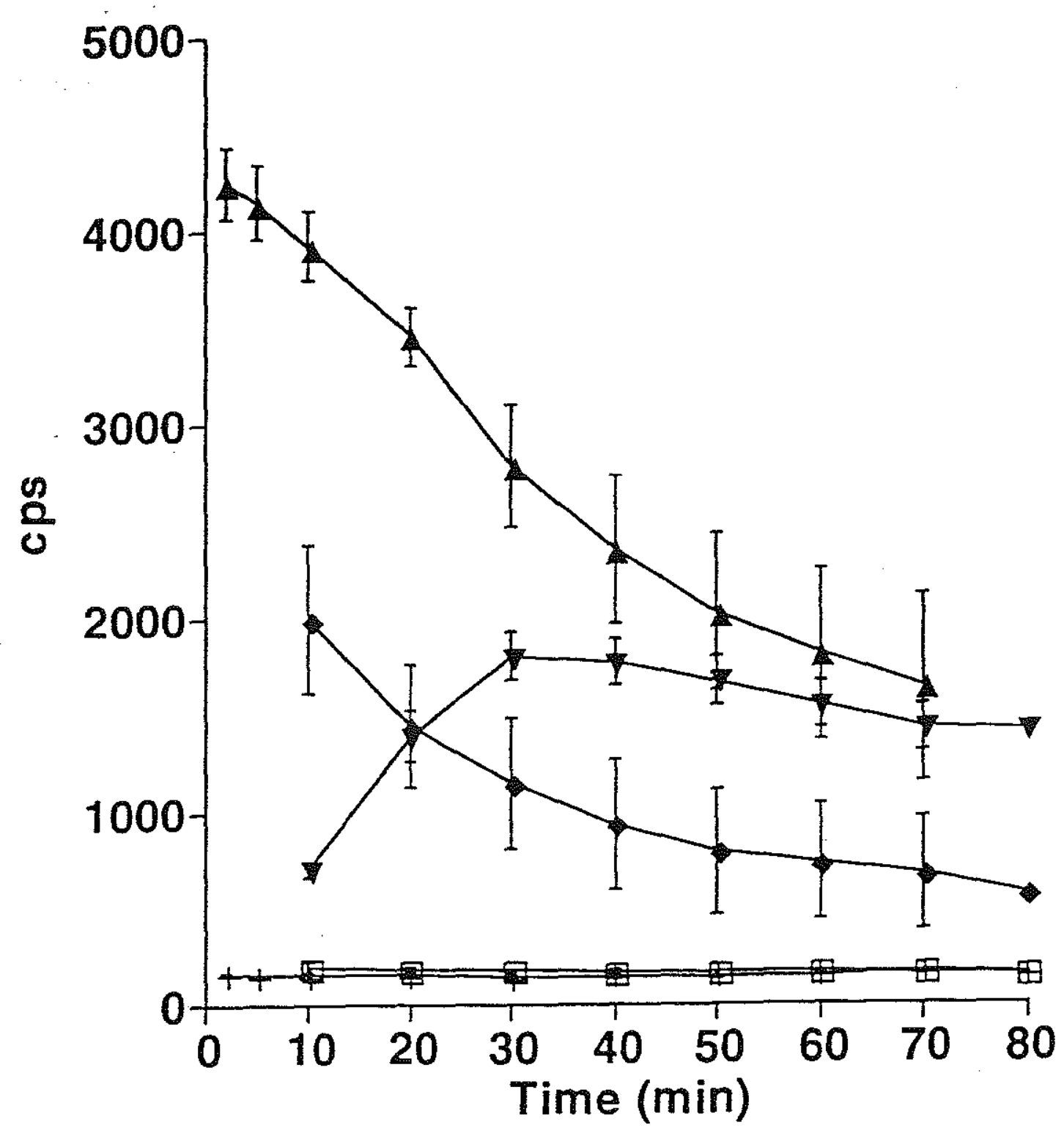

Figure 2.10 


\section{ChAPTER 3 - ALISS (Ambient Light Imaging and Spectral System)}

Light emission from hydrothermal vents must be well characterized both spectrally and spatially in order to determine which of the mechanisms discussed in the previous chapter are likely sources (or at least, which are not likely sources). The ALISS camera system was designed to do just that. Unlike the previous OPUS instrument, ALISS is able to obtain both spectral and spatial information by imaging vents in a number of wavelength bands simultaneously. Taking a number of short-exposure images provides temporal information. By using a CCD camera, long exposure times, and image processing techniques, even very low light levels can be measured.

\subsection{INSTRUMENTATION}

\subsubsection{CCD TECHNOLOGY}

In 1969, the charge-coupled device (CCD) was invented - initially as a storage device — by Willard Boyle and George Smith at Bell Labs in Murray Hill, New Jersey [Boyle and Smith, 1970]. Both Buil [1991] and McLean [1997] provide an overview of the principles behind CCD imaging. The basic unit of a CCD is the MOS (metal-oxidesemiconductor) capacitor - a doped (usually p-type) semiconductor (silicon) connected to a number of metal electrodes (or "gates") through a thin insulating layer (silicon oxide). When a voltage is applied to the gate, an electrostatic "potential well" is created in the semiconductor which can store electrons. The energy from an incoming photon releases an electron within the silicon which can then be stored in the potential well. The CCD chip is a grid of these potential wells or pixels (picture elements) - 1024 x 1024 in the case of ALISS. Each pixel contains two or more MOS capacitors. The voltages applied to the capacitors are manipulated to transfer the charges from one pixel to the next (hence the 
name "charge-coupled" device) (Figure 3.1). The charges are transferred horizontally to the output (or serial) register which transfers them, in the same manner, vertically to the output amplifier (Figure 3.2). Electrons can also be released by heat energy (even at room temperature) creating a thermal signal and noise that is unrelated to the incident radiation. This can be reduced by significantly cooling the $\mathrm{CCD}$, such as with liquid nitrogen or a thermoelectric cooler.

A front illuminated CCD chip has photons incident on the electrode side of the chip. Thus, the photons must pass through the electrode structure before reaching the storage region of the silicon. This limits the quantum efficiency of the CCD, particularly in the blue region, where absorption of blue light in the electrode structure can lead to almost a complete lack of response. Thinning the silicon substrate and illuminating the chip from the back greatly improves the quantum efficiency, especially in the blue region (Figure 3.3). However, because red light needs to pass through more silicon than blue before it is absorbed, if the chip is thinned too much it will lose its red response. Anti-reflection coating can also be applied to the surface of the chip to improve the quantum efficiency.

CCD's were first used for astronomical imaging in 1976. The features that make them attractive to astronomers also make them useful for imaging deep-sea hydrothermal vents. CCD's are small, low-power devices with excellent sensitivity and linearity — over wide ranges in both wavelength and light level. Very faint objects (stars or hydrothermal vents) can be imaged by using long exposure times to build up charge in the pixels. Thus, it is an inherently integrating device. The excellent sensitivity also allows short exposures to be obtained with sufficient signal to observe temporal variations in light emission. The digital image captured by the CCD can be processed and analyzed by computer to extract data (such as variations or low intensity levels) imperceptible to the naked eye. The image processing steps will be discussed later in this chapter. 


\subsubsection{ALISS CAMERA DESIGN}

ALISS is a PentaMAX camera built by Princeton Instruments using a SITe $1024 \mathrm{x}$ 1024 back-illuminated CCD chip. Each pixel is $24 \times 24 \mu \mathrm{m}$. The chip has a peak quantum efficiency of $80 \%$ at $\sim 640 \mathrm{~nm}$ (Figure 3.3). Cooling of the $\mathrm{CCD}$ (up to $50^{\circ} \mathrm{C}$ below the surrounding water temperature) is provided by a thermoelectric cooler which is coupled to the titanium pressure case to dissipate heat to the surrounding seawater $\left(2^{\circ} \mathrm{C}\right.$ ambient). The camera has a dynamic range of 16 bits (i.e., a maximum of 65,536 counts), and a fixed gain of 6.1 electrons per count. A specially designed nine-lens optical assembly (designed and built by Truax Associates) divides the $1024 \times 1024$ pixel image into nine identical $\sim 300 \times 300$ pixel images (hereafter referred to as "tiles"). The optical assembly, attached in front of the CCD chip, consists of three main elements - a field flattener, the nine-lens optic block, and the filter arrays (Figure 3.4). The field flattener and a set of baffles (to prevent cross talk between channels) are mounted directly over the CCD chip which is housed in an evacuated chamber (Figure 3.5A). Nine $6 \mathrm{~mm}$ diameter triplet lenses are housed in an aluminum optic block just outside of the vacuum chamber (Figure 3.5B). Each triplet lens was individually aligned and focused so that all image the same object at a focal distance of $50 \mathrm{~cm}$ in seawater. At this distance, each lens subtends a solid angle of $4.5 \times 10^{-5}$ steradians and has a $16 \times 16 \mathrm{~cm}$ field of view. Fine focusing is achieved by moving the optic block in and out with a small pico-motor. Two filter arrays, containing nine filters each (Figure 3.5B), are located directly in front of the optic block and can be switched by two solenoids. Eleven of the filters are $\sim 100 \mathrm{~nm}$ and six are $\sim 50$ $\mathrm{nm}$ in bandwidth. The filter bands overlap to provide complete coverage of the spectrum from $400 \mathrm{~nm}$ to $1000 \mathrm{~nm}$. The eighteenth filter is $10 \mathrm{~nm}$ in bandwidth and is centered on $589 \mathrm{~nm}$. 
The CCD and its accompanying optics and electronics are housed in a 7.5 inch diameter $6 \mathrm{Al}-4 \mathrm{~V}$ titanium pressure case. The optical endcap contains a glass viewport that is a 2 inch thick, $62^{\circ}$ sector of a dome with a 4 inch internal radius (Figure 3.4). The connector endcap is a flat plate design with a commercial 12-pin connector (D. G. O'Brien, Inc.). The pressure case was tested to 10,000 psi [Goldsborough et al., 1998]. The camera is powered by 120 volts at $\sim 1$ ampere provided by the submersible Alvin.

\subsubsection{CALIBRATION}

ALISS was calibrated at the California Institute of Technology (Caltech) following the 1997 EPR cruise. The Palomar Observatory group at Caltech has a Quantum Efficiency (QE) Testing System used to calibrate CCD cameras used for astronomy. The QE Test device consists of a broadband light source (an Oriel 66002 deuterium lamp), a system of optics that constrains the wavelength and bandwidth of the output beam, and a $30 \mathrm{~cm} \mathrm{x} 40$ $\mathrm{cm} \times 1.5 \mathrm{~m}$ black anodized light box. A rotating mirror within the box is used to switch the beam between an NBS-traceable silicon photodiode $\left(1 \mathrm{~cm}^{2}\right.$ active area) and the instrument to be calibrated. The photodiode signal is measured with a Melles-Griot Large Dynamic Range Amplifier unit (13AMP003) [Behr, 1996]. ALISS was mounted to the light box opposite the photodiode, and was packed in ice to prevent the camera from overheating in its pressure case.

Measurements were made every $10 \mathrm{~nm}$ from $400 \mathrm{~nm}$ to $1040 \mathrm{~nm}$, providing five to ten calibration points per filter. The output and background level of the photodiode (in Amps) at each wavelength were recorded both before and after imaging with the ALISS camera. These photodiode values were averaged and converted to Watts using the photodiode calibration sheet (Figure 3.6). Dividing this value by the $1 \mathrm{~cm}^{2}$ active area, and 
the energy of a photon at that wavelength gives the photon flux (photons $/ \mathrm{cm}^{2} / \mathrm{sec}$ ) of the light source. The photon energy is determined by

$$
E=h \cdot c / \lambda
$$

were $h$ is Planck's constant $\left(6.626 \times 10^{-34} \mathrm{~J} \cdot \mathrm{s}\right), c$ is the speed of light in vacuo $\left(\sim 3.0 \times 10^{8}\right.$ $\mathrm{m} / \mathrm{sec})$, and $\lambda$ is wavelength in meters.

The signal in the ALISS image was summed, divided by the exposure time, and multiplied by the gain ( 6.1 electrons/count) to determine the electrons/sec measured by the ALISS camera. The electrons/sec measured by ALISS was divided by the photons $/ \mathrm{cm}^{2} / \mathrm{sec}$ incident on ALISS's pressure window (as measured by the calibrated photodiode and adjusted for difference in distance from the light source) to determine the effective aperture (area) of the ALISS camera system in air (which includes the entrance pupil of the lens, the transmission through the filter, and the QE of the chip). The effective aperture is smaller in seawater than in air by a factor of $\sim 1.77$ (the square of the index of refraction of seawater) [G. Reynolds and B. Truax, per. comm.]. To account for the smaller entrance pupil area when imaging in water rather than air, the effective aperture was divided by 1.77 . The effective aperture in seawater for each filter is shown in Figure 3.7. The falling off of the effective aperture at long wavelengths is due to the decreasing QE of the CCD (see Figure 3.3).

\subsubsection{OPERATIONS}

The ALISS camera was deployed at two vent sites in the Pacific: the Venture Hydrothermal Field ( $9^{\circ} \mathrm{N}$ EPR) and the Main Endeavour Field on the Juan de Fuca Ridge. ALISS was mounted in the Alvin science basket on a frame which could be raised and tilted with two hydraulic rams (Figure 3.8). The camera itself could be panned left and right 
with the Alvin manipulator. A scientist inside the submersible controlled the camera using the ALISS computer. The WinView acquisition software (provided by Princeton Instruments) allowed the scientist to adjust exposure time, set the shutter position, and acquire and save images. Macros were added to the program to control focusing and filter swapping.

There are two important issues to consider when selecting a vent to image: distance and stability. To ensure that the object is in focus, the ALISS camera must be positioned $50 \mathrm{~cm}$ from the vent (as indicated by two lasers which cross at that distance) (Figure 3.9). Chimney structures can vary greatly both from site to site and within a site. In some cases venting occurs atop a chimney structure, and in other cases venting occurs at the base or on the sides of structures. Sulfide structures can be large and stable, such as those seen on the Juan de Fuca Ridge, or small and fragile, like many of the chimneys at $9^{\circ} \mathrm{N}$ on the East Pacific Rise. Thus, only some vents have a geometry that allows ALISS to be properly positioned for imaging. In addition to putting the vent at the proper focal distance and within the camera's field of view, the submarine must remain stationary during the imaging. This is accomplished either by driving the submarine against a sturdy sulfide structure, or setting the submarine on a ledge or the seafloor. This further limits which vents are suitable for ALISS work.

The ALISS camera must be powered on at least 20 minutes before imaging to allow time for the thermoelectric cooler to stabilize the CCD temperature. During ambient light imaging all viewports must be blacked out and all external lights secured. Each ALISS exposure was nominally 5 minutes long, and two to seven images were acquired with each filter array. The length of the exposure time, and the number of exposures were a trade-off between the need to achieve a high signal-to-noise ratio and the need of the pilot to safely operate the submarine. Due to the low light levels at hydrothermal vents, long exposure 
times were needed to acquire a large signal. However, working in such close proximity to these vents with Alvin's lights off and viewports blacked out is dangerous. The hightemperature fluids exiting the vents are hot enough to melt Alvin's pressure windows. Thus, the pilot must continually check on the position of the submarine and the condition of the venting structure. By taking a number of 5 minute images, an adequate signal was obtained and the pilot was able to verify the submarine's stability between images. Median averaging three or more images also helped greatly in reducing noise. In some cases, movement by passengers caused the submarine to shift, creating blurred images. In other cases, the material used to black out the viewports was not properly situated or moved during imaging, possibly causing a light leak from the sub. Any images thought to be affected in these ways were not used for analysis.

In addition to ambient light images, a number of other images were obtained for use in correcting systematic errors, which is discussed in the image processing section below. These images include bias images, dark images, and flat field images (Figure 3.10). A bias image is an image obtained by simply reading the CCD chip without any signal acquired. That is, the shutter is closed and the exposure time is set to zero. There is some structure to this image which can change every time the power is cycled on the camera. Ideally, a large number of bias images should be collected on each dive. This was not accomplished during the first two ALISS deployments, but should be done on subsequent dive cruises.

During the dives, dark images were taken before, after, and during imaging of the vent. These dark images had exposure times of 5 minutes (the same as the ambient light images) and were acquired with the shutter closed. The signal in the dark image is due solely to thermal effects causing the build up of charge as discussed above (dark counts).

The pixels in a CCD are not completely uniform. That is, each pixel will have a slightly different response from the next for the same incident illumination. In order to 
correct for this variation in effective pixel $\mathrm{QE}$, a flat field image (hereafter referred to as a "flat") is need. A flat is obtained by evenly illuminating the CCD chip and acquiring an image. Ideally, flats should be acquired at depth and the camera should remain on throughout the dive, as cycling the power can cause slight changes in the response of the camera. However, a manner of evenly illuminating the $\mathrm{CCD}$ while on the seafloor has not yet been developed and thus, flats were obtained prior to each Alvin dive while the submarine was on deck. Even illumination was achieved by covering the ALISS window with a thick, translucent endcap and using ambient sunlight as the light source. The endcap was masked to allow illumination only within ALISS's field of view, as extraneous light outside of the field of view can cause odd reflections and light leakage across channels. During the second dive series (to the Main Endeavour Field) a covering of one layer of black plastic was added to the endcap to allow longer exposure times to prevent shutter vignetting effects (which are a problem below $50 \mathrm{msec}$.). Shutter vignetting causes a shadow around the edges of a short-exposure image because the shutter requires a finite amount of time to open and close. Exposure times were chosen to ensure that the signal was high enough in each channel to be in the linear response region of the $\mathrm{CCD}$, and varied from day to day depending on the ambient lighting conditions. Five flat field images were acquired with each filter array prior to each dive.

\subsection{Data Analysis}

\subsubsection{IMAGE PROCESSING}

The ALISS images were processed using the Image Reduction and Analysis Facility (IRAF - distributed by the National Optical Astronomy Observatories) and Interactive Data Language (IDL — distributed by Research Systems, Inc.) image processing packages. When imaging at low-light levels, there are a number of systematic 
errors which can dominate the background noise. These systematics are both additive and multiplicative, and must be removed to perform accurate photometry on the images [Tyson, 1986; Tyson, 1990]. The image reduction steps are as follows (Figure 3.10):

\section{(1) Subtract DC bias level}

All images contain an overscan strip which is obtained by reading 20 more columns than exist on the CCD array. This overscan strip provides the DC bias level. The first step in processing the images is removing the bias level and trimming the overscan strip. The bias level is determined by calculating the mean of the overscan strip, and then subtracting this value from every pixel. This is performed on all ALISS images - biases, darks, flats, and ambient light images.

\section{(2) Subtract bias structure}

A "master bias" image should be created for each dive by averaging a number of bias images from that dive. This is then subtracted from all images obtained on that dive - darks, flats, and ambient light images. However, a useful number of bias images were not collected during the dives. Therefore, the master bias image was extracted from the dark images for use in processing the data. The dominant feature in the bias image was a "ringing" associated with the CCD electronics. This master bias was compared to bias images obtained in the lab subsequent to the last cruise, and was determined to be acceptable for image processing.

\section{(3) Subtract dark charge}

All of the dark images acquired during a cruise were averaged and scaled to create a one-second "master dark" image for each cruise. The ambient light images 
and flats were scaled to one-second, and the master dark was subtracted from each image to remove thermal effects.

(4)

\section{Median averaging}

All of the vent images from one filter array were then median averaged to reduce noise. When median averaging the values for one pixel, $\sigma$ (the standard deviation of the values for that pixel) varied from $\sim 0.01$ in the background aperture ( $\sim 0.01$ counts/second) to $\sim 0.7$ in the source aperture (maximum of $\sim 1-3$ counts/second).

(5) Divide by flat field

Flat field corrections, which correct for pixel-to-pixel variations, were made by dividing each image by a "master flat". The master flats (one for each filter array) were made by averaging the flats obtained prior to each dive and scaling the result by the mean signal to produce an image with a mean value of one. Given the variations in intensity for each filter, each tile was scaled individually. During the image processing of the vent images, the flat field correction was made on a tileby-tile basis. An error image was also created for each flat by assuming the noise, $\sigma_{f f}$ to be half of the minimum-to-maximum range for each pixel from the five images. This error image was used in calculating the total noise in the image.

Once the vent images were fully processed as described above, a source mask and complementary background mask were created using the channel with the highest signal-tonoise ratio (the $870 \mathrm{~nm}$ filter) to isolate the area of light emission from the background. The same mask was applied to all eighteen channels and count rates per second (minus the background levels scaled to the number of pixels in the source mask) were calculated for all 
filters. These values were then multiplied by the gain (6.1 electrons/count) and divided by the source area giving the detected photon flux at the ALISS CCD in photons $/ \mathrm{cm}^{2} / \mathrm{sec}$. Analysis of specific parts of the plume could be performed by limiting the source mask to a certain region. Also, the source mask could be limited to intensities within a specific range (i.e., from $0-100 \%$ of the intensity values measured).

The ALISS data were then be compared to the flux expected from a theoretical black body (calculated using Planck's equation for a given temperature and emissivity) attenuated through $50 \mathrm{~cm}$ of water, corrected with the calibration data, and summed over each filter.

\subsubsection{NOISE}

Noise is the random variation of a signal about an average value. ALISS data is subject to noise inherent in the camera system. The dominant sources of inherent CCD noise include (1) thermal noise associated with the dark current, (2) photon noise associated with the signal, and (3) readout noise associated with the electronics. As discussed above, thermally produced charges that build up on a CCD over time can be limited by cooling the CCD. While the mean thermal signal can be removed from an image by simple subtraction, the noise associated with it cannot. The RMS noise $\left(\sigma_{t}\right)$ of the thermal signal is given by

$$
\sigma_{t}=\sqrt{S_{t}}
$$

where $S_{t}$ is the number of charges (electrons) produced thermally. Thus, cooling the CCD reduces both dark charge and thermal noise. The dark noise of the ALISS images is approximately 1-2 electrons/second. 
The build up of signal on a CCD chip by incoming photons, can be described as a Poisson process. As such, the variance is given by the mean of the average signal. The photon (or "shot") noise $\left(\sigma_{p h}\right)$ is given by

$$
\sigma_{p h}=\sqrt{S_{p h}}
$$

where $S_{p h}$ is the incoming signal in electrons. Thus, the greater the incoming signal, the greater the photon noise.

The readout noise $\left(\sigma_{\text {read }}\right)$, unlike the previous two, does not originate in the imaging area of the CCD chip. This noise is generated in the output preamplifier. It can be reduced by cooling of the CCD, or decreasing the readout rate. The readout noise of the ALISS camera (provided by Princeton Instruments) is 12 electrons per pixel. It is the combination of the dark noise and the readout noise that determine the effective detection limit of the camera. The total noise of the processed image is the quadratic sum of all of the noise components.

$$
\sigma_{\text {total }}=\sqrt{\sigma_{\text {dark }}+\sigma_{p h}+\sigma_{\text {read }}+\sigma_{f f}}
$$




\section{Figure Captions}

Figure 3.1 Diagram of charge-coupling in a three-phase CCD. Applying a voltage to a gate (electrode) generates a depleted region to store charge. By coupling gates, electrons can be moved from one gate to another. [Modified from McLean, 1997]

Figure 3.2 Schematic view showing the basic layout of a three-phase CCD. Each pixel consists of three electrodes (stippled, checked, and flecked patterns). By adjusting voltages of the electrodes, charges can be moved across the CCD while being kept isolated from the charge in adjacent pixels. Charges are moved (as shown in Figure 3.1) horizontally to the serial register and then vertically to the output amplifier. [Modified from McLean, 1997]

Figure 3.3 Quantum efficiency (QE) plot of front and back illuminated CCD's. The heavy line indicates the response of the back-illuminated chip that is part of the ALISS camera. [From Princeton Instruments]

Figure 3.4 Schematic of the ALISS optical assembly. The CCD chip and field flattener are housed within an evacuated chamber. The nine lenses and filter arrays are located just outside of the vacuum chamber. The distance from the filter array to the optical window is approximately 3.7 inches.

Figure 3.5 ALISS components - A) Field flattener with baffles (front left) before being mounted over the CCD chip (right). B) Optic block containing nine triplet lenses (right) and filter arrays (left).

Figure 3.6 Calibration sheet for the photodiode in the Caltech QE Test System used to calibrate the ALISS camera. [From the Palomar Group at Caltech] 
Figure 3.7 Calibration curves for the 18 ALISS filters. The effective aperture $\left(\mathrm{cm}^{2}\right)$ includes entrance pupil of the lens, transmission of the filter, $\mathrm{QE}$ of the CCD chip and any other internal losses in the optical assembly. The effective aperture determined in air was divided by the 1.77 (the square of the index of refraction of water) to account for the smaller entrance pupil when used in water rather than air.

Figure 3.8 ALISS camera mounted in Alvin science basket. The pilot's viewport can be seen at the top of the picture.

Figure 3.9 ALISS at L vent on the East Pacific Rise as photographed through the pilot's viewport. ALISS is in the lower right of the image. The red dot on plume in front of the ALISS camera is the intersection of the ranging lasers indicating a distance of $50 \mathrm{~cm}$.

Figure 3.10 Images obtained with the ALISS camera and how they are used in processing the data. A raw ALISS image contains 20 extra columns referred to as an "overscan strip" - which give the bias level of the CCD. Bias images, dark images, and flat field images must all be obtained to correct for systematic errors during image processing. 
one pixel

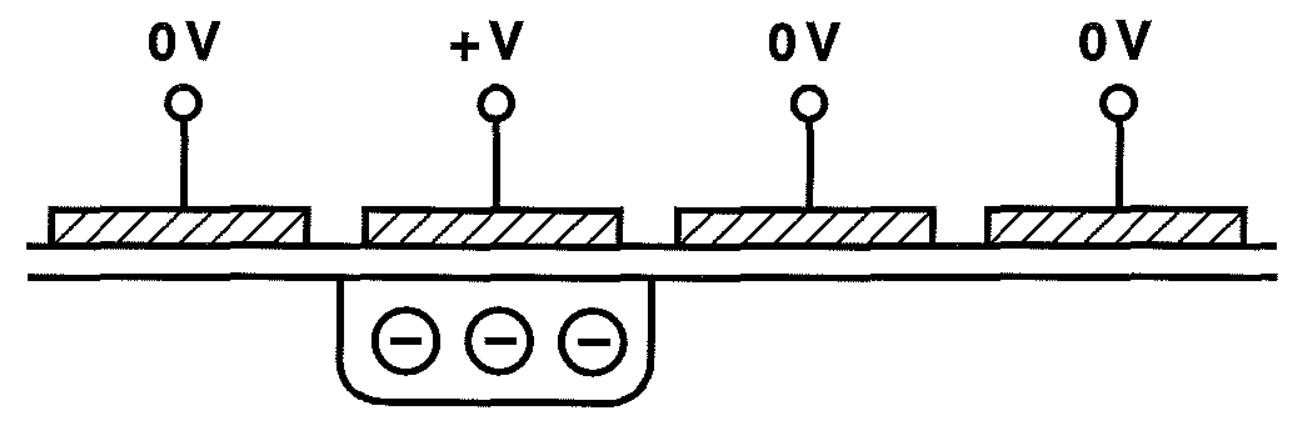

one pixel

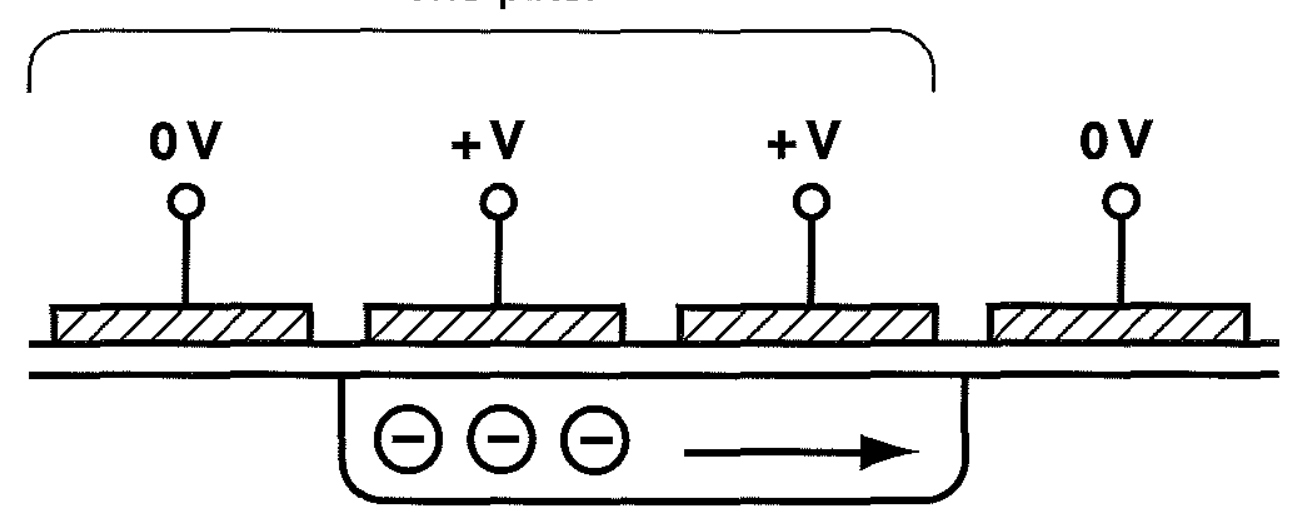

one pixel

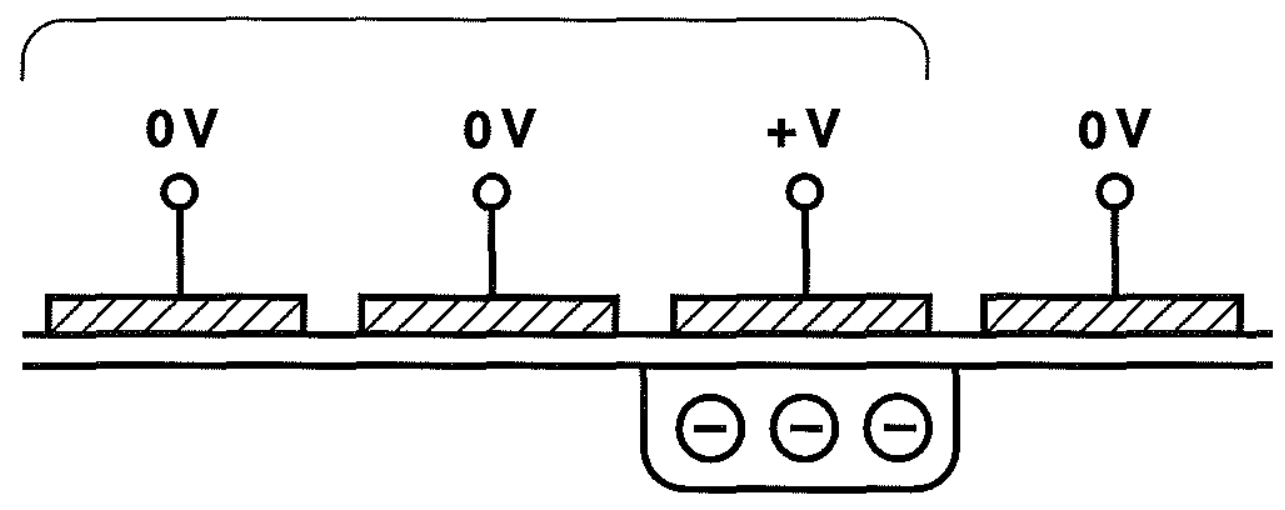

Figure 3.1 


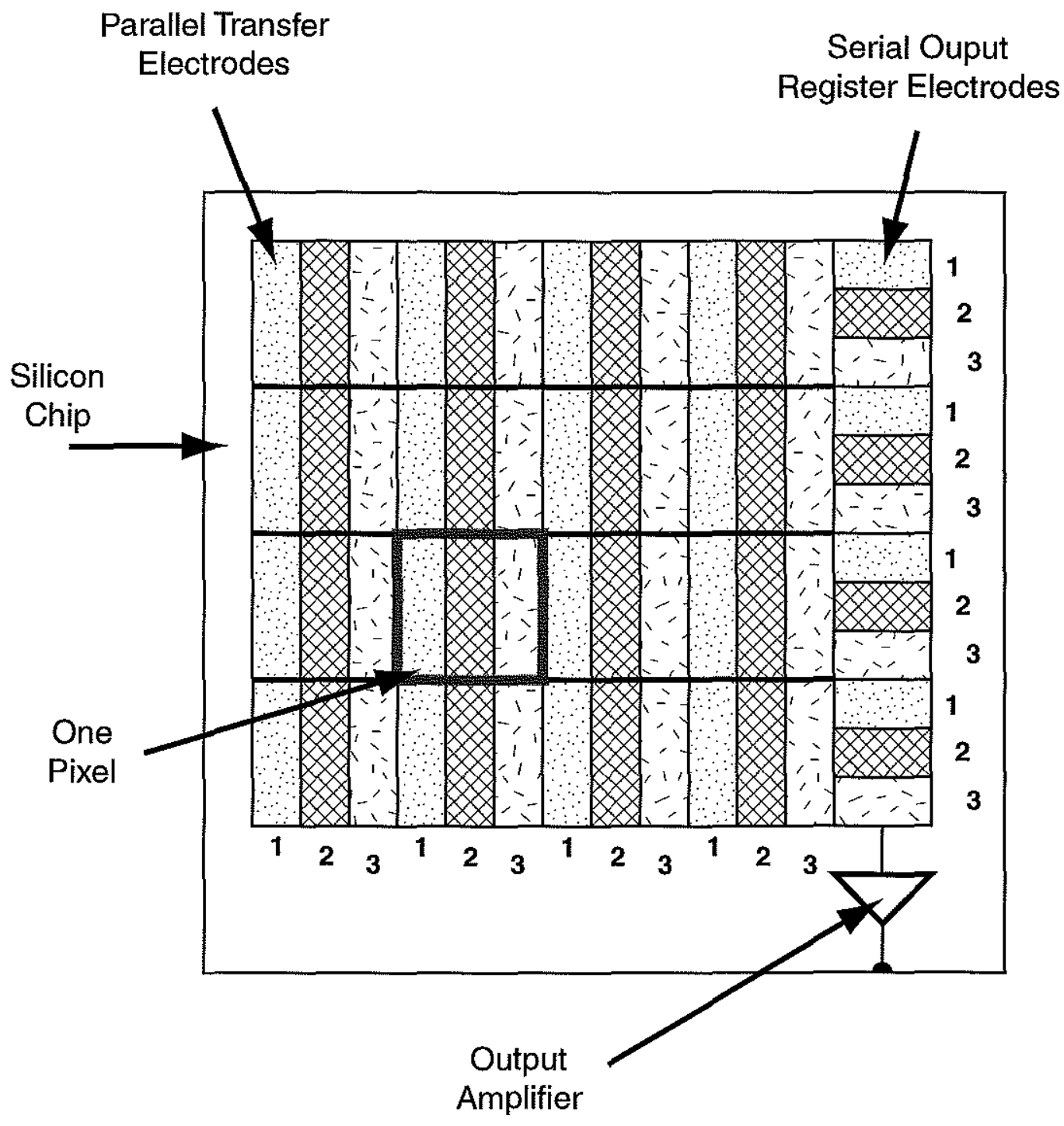

Figure 3.2 


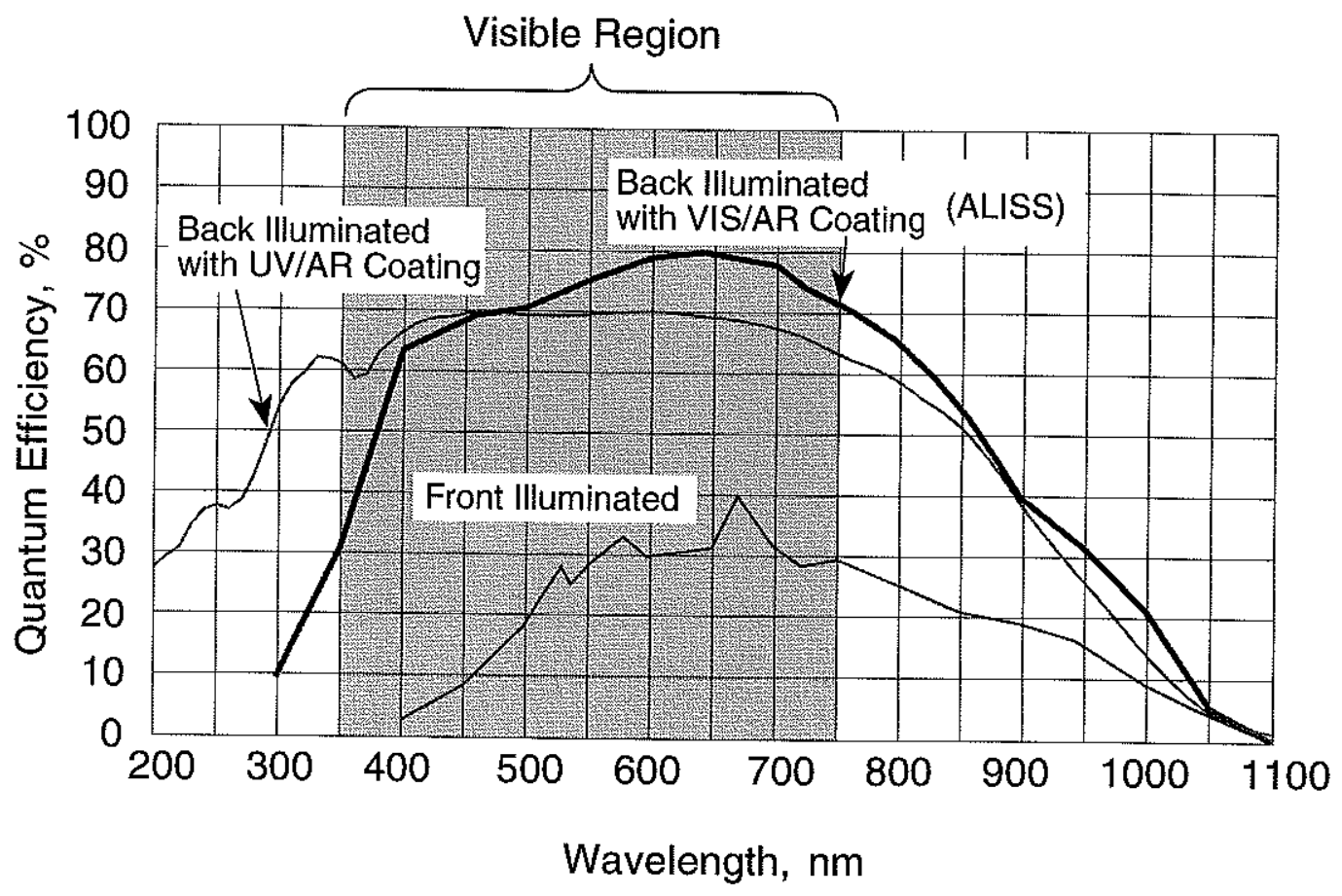

Figure 3.3 


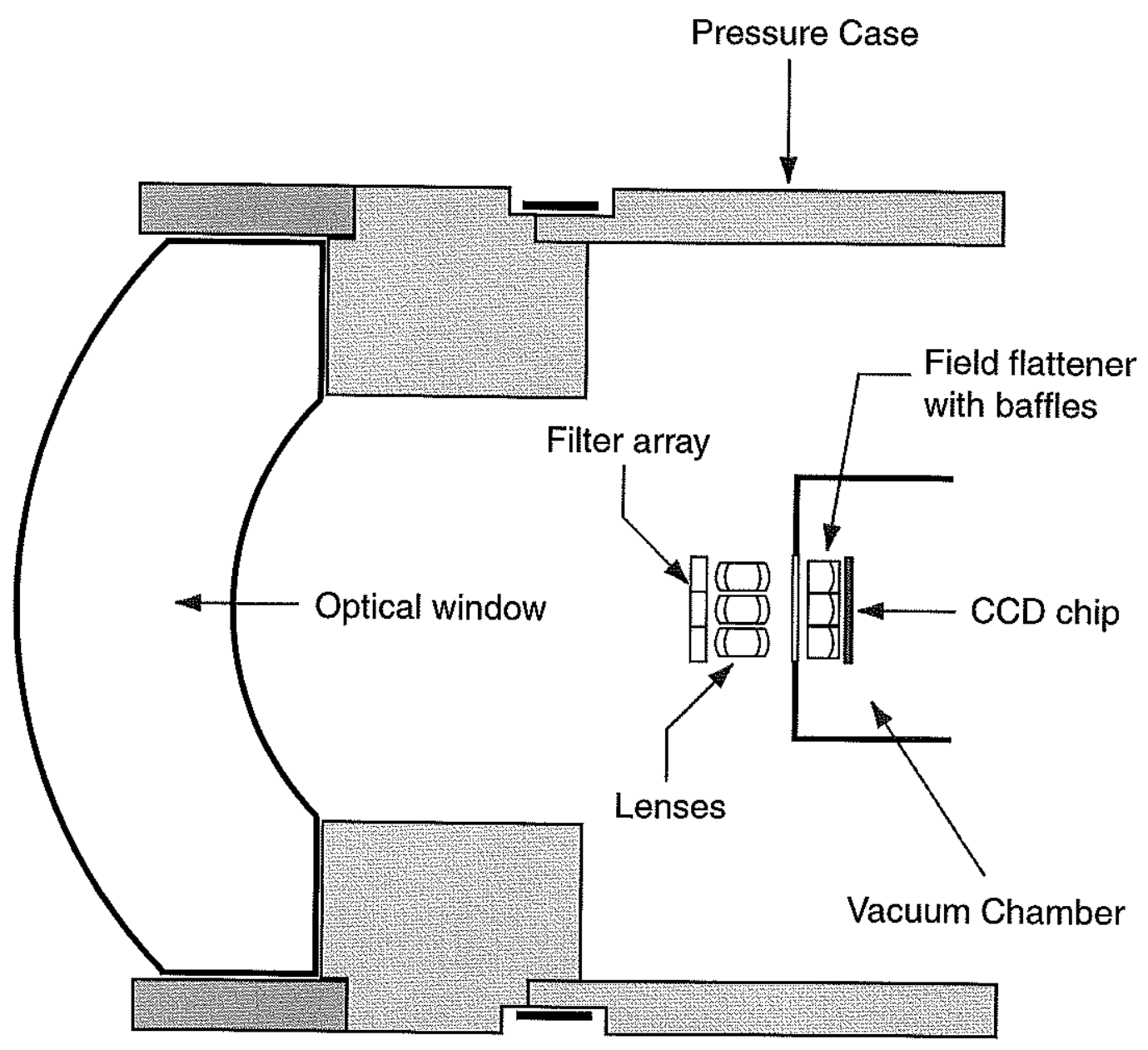

Figure 3.4 

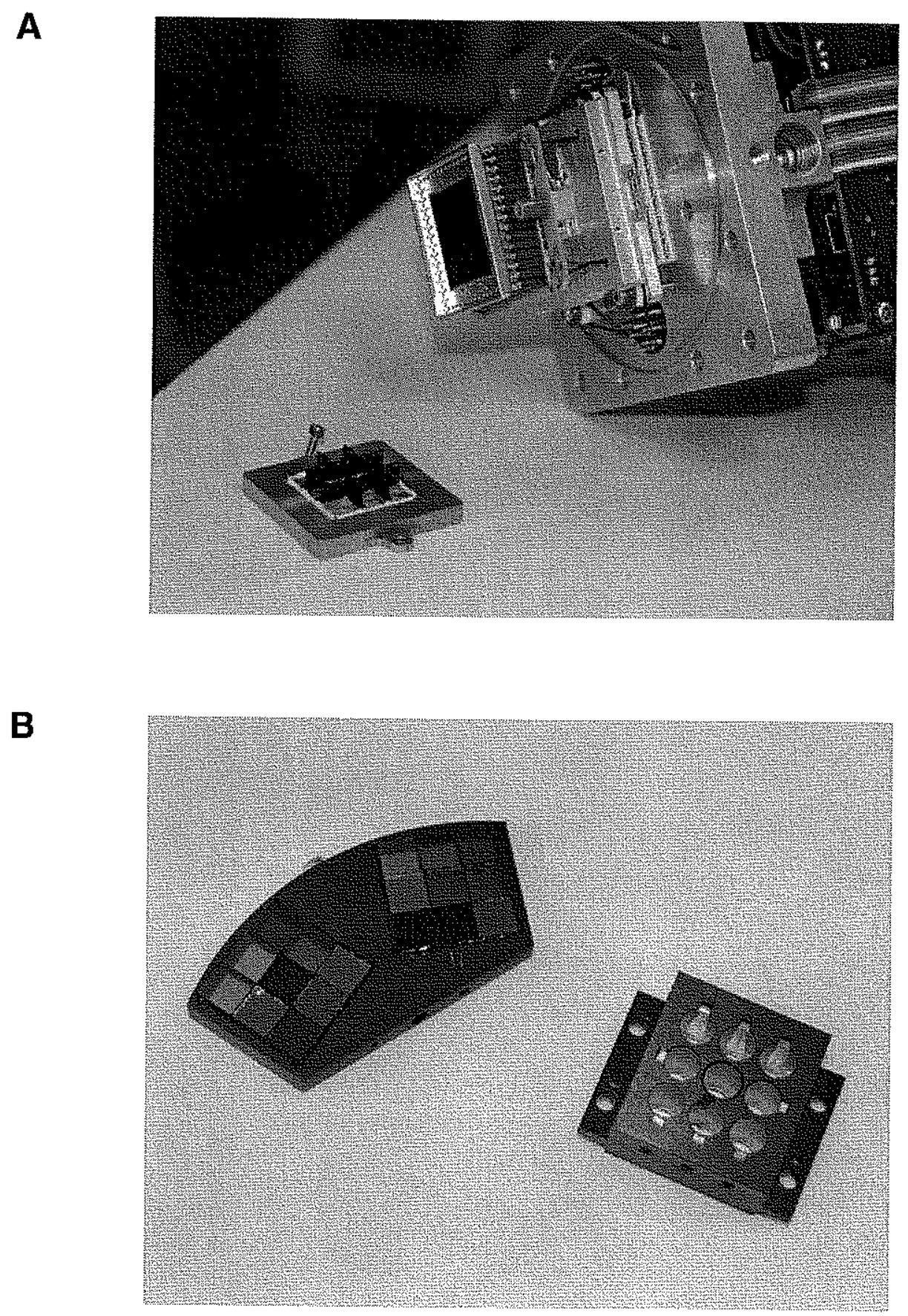

Figure 3.5 


\section{CERTIFICATE OF CALIBRATION}

SLUCON PHOTODIODE

MODEL NUMBER PIN 1ODP, SERIAL NUMBER 1937

CALIFORNIA INSTITUTE of TECH.

\begin{tabular}{|c|c|}
\hline $\begin{array}{l}\text { WAVL } \\
\text { nm }\end{array}$ & $\begin{array}{l}\text { RESP } \\
\text { AW }\end{array}$ \\
\hline 350 & 0.0555 \\
\hline 360 & 0.0561 \\
\hline 370 & 0.0608 \\
\hline 380 & 0.0707 \\
\hline 390 & 0.0862 \\
\hline 400 & 0.1030 \\
\hline 410 & 0.1205 \\
\hline 420 & 0.1387 \\
\hline 430 & 0.1564 \\
\hline 440 & 0.9742 \\
\hline 450 & 0.1949 \\
\hline 460 & 0.2087 \\
\hline 470 & 0.2249 \\
\hline 480 & 0.2410 \\
\hline 490 & 0.2561 \\
\hline 500 & 0.2707 \\
\hline 510 & 0.2849 \\
\hline 520 & 0.2982 \\
\hline 530 & 0.3112 \\
\hline
\end{tabular}

\begin{tabular}{|c|c|}
\hline $\begin{array}{l}\text { WAVE } \\
\text { nmin }\end{array}$ & $\begin{array}{l}\text { RESP } \\
\text { AW }\end{array}$ \\
\hline 540 & 0.3236 \\
\hline 550 & 0.3352 \\
\hline 560 & 0.3465 \\
\hline 570 & 0.3574 \\
\hline 580 & 0.3675 \\
\hline 590 & 0.3774 \\
\hline 600 & 0.3867 \\
\hline 610 & 0.3956 \\
\hline 620 & 0.4044 \\
\hline 630 & 0.4131 \\
\hline 640 & 0.4189 \\
\hline 650 & 0.4288 \\
\hline 660 & 0.4363 \\
\hline 670 & 0.4436 \\
\hline 680 & 0.4509 \\
\hline 690 & 0.4578 \\
\hline 700 & 0.4645 \\
\hline 710 & 0.4713 \\
\hline 720 & 0.4777 \\
\hline
\end{tabular}

WAVE RESP

WAVE RESP:

\begin{tabular}{|r|r|}
\hline 730 & 0.4841 \\
\hline
\end{tabular}

\begin{tabular}{|r|r|}
\hline 740 & 0.4900 \\
\hline 750 & 0.4958 \\
\hline 760 & 0.5019 \\
\hline 770 & 0.5084 \\
\hline 780 & 0.5114 \\
\hline 790 & 0.5175 \\
\hline 800 & 0.5234 \\
\hline 810 & 0.5288 \\
\hline 820 & 0.5341 \\
\hline 830 & 0.5394 \\
\hline 840 & 0.5439 \\
\hline 850 & 0.5492 \\
\hline 860 & 0.5540 \\
\hline 870 & 0.5584 \\
\hline 880 & 0.5626 \\
\hline 890 & 0.5670 \\
\hline 900 & 0.5710 \\
\hline 910 & 0.5757 \\
\hline
\end{tabular}

\begin{tabular}{|r|r|}
\hline 920 & 0.5794 \\
\hline 930 & 0.5829 \\
\hline 940 & 0.5856 \\
\hline 950 & 0.5879 \\
\hline 960 & 0.5888 \\
\hline 970 & 0.5872 \\
\hline 980 & 0.5832 \\
\hline 990 & 0.5750 \\
\hline 1000 & 0.5601 \\
\hline 1010 & 0.5366 \\
\hline 1020 & 0.5041 \\
\hline 1030 & 0.4590 \\
\hline 1040 & 0.4081 \\
\hline 1050 & 0.3426 \\
\hline 1060 & 0.2793 \\
\hline 1070 & 0.2247 \\
\hline 1080 & 0.1841 \\
\hline 1090 & 0.1489 \\
\hline 1100 & 0.1190 \\
\hline
\end{tabular}

(2)

\section{SPECTRAL RESPONSIVITY}

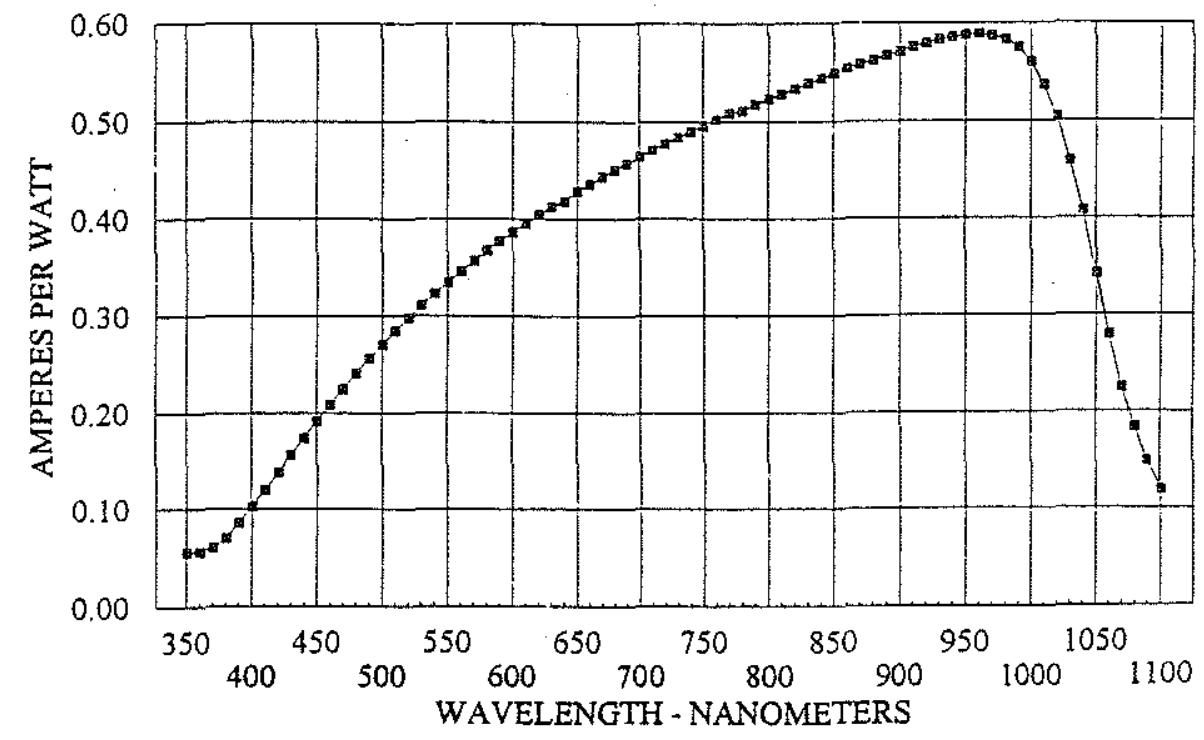

Figure 3.6 
Effective Aperture of ALISS Filters

$100 \mathrm{~nm}$ bandwith filters

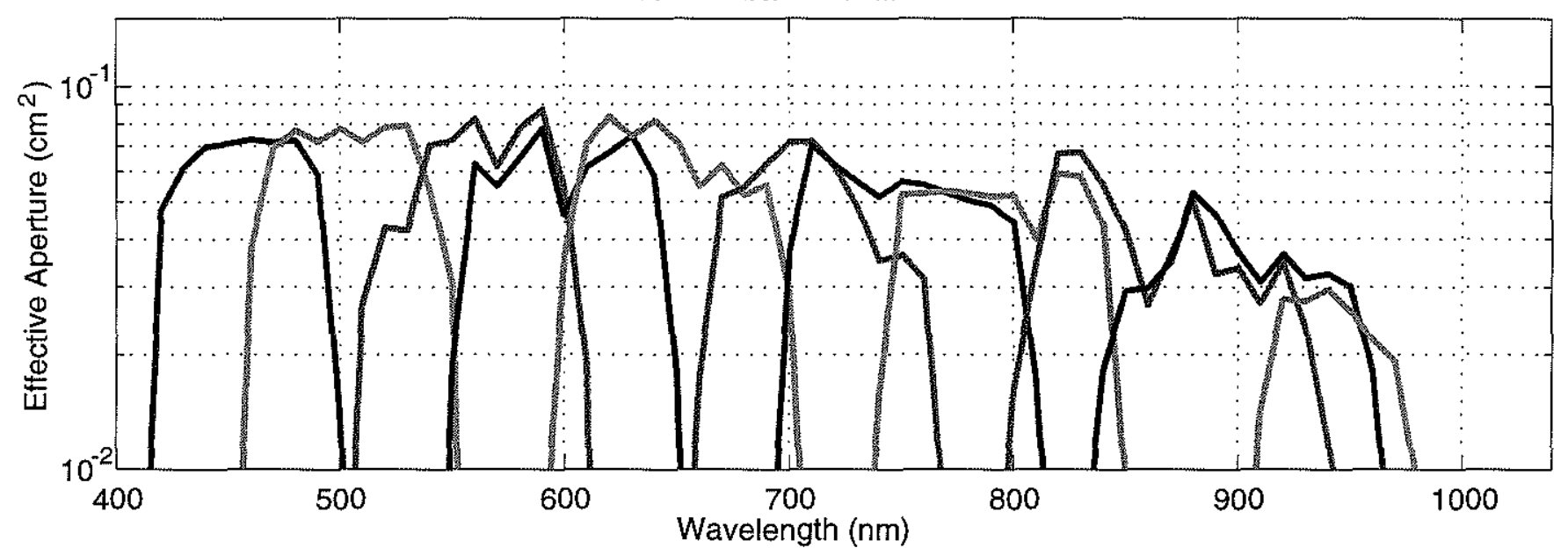

$50 \mathrm{~nm}$ \& $10 \mathrm{~nm}$ bandwith filters

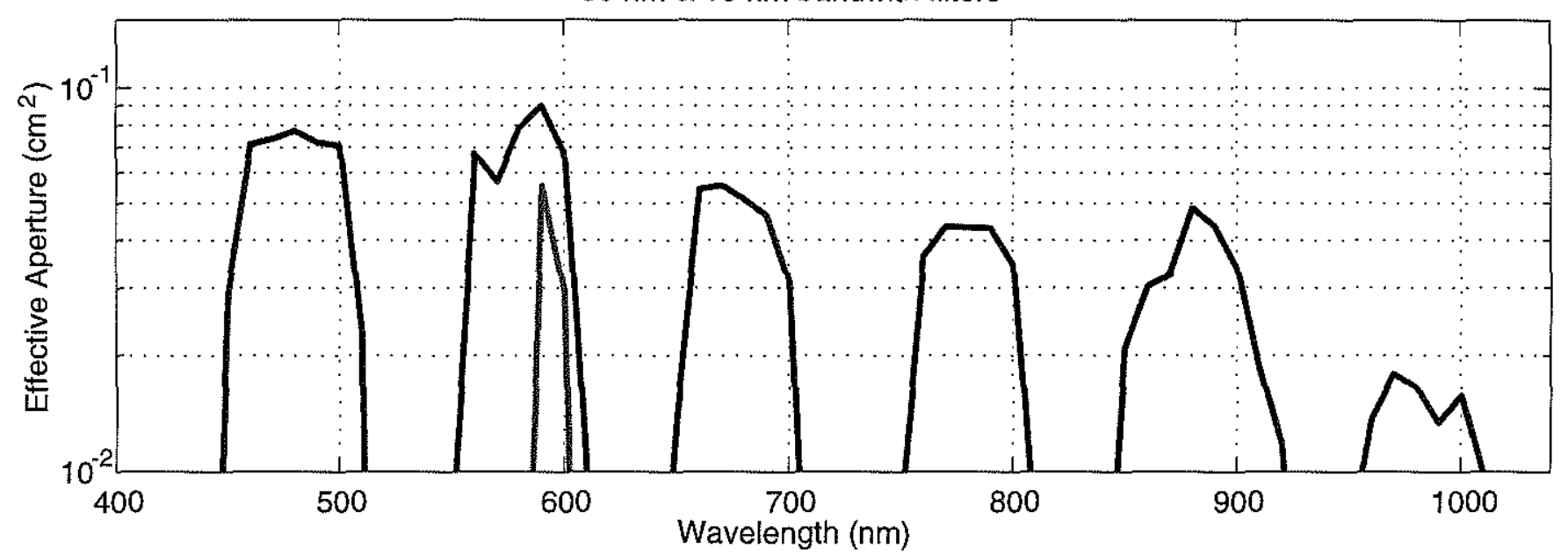

Figure 3.7 


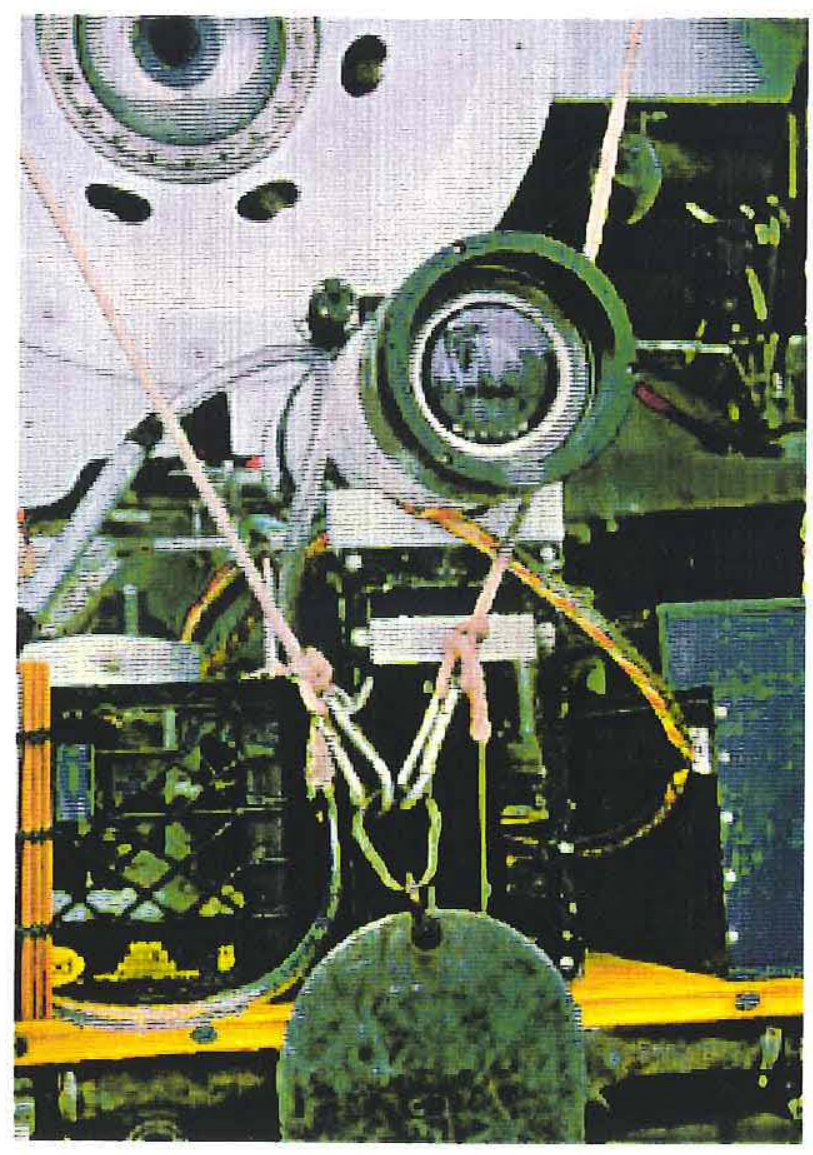

Figure 3.8 


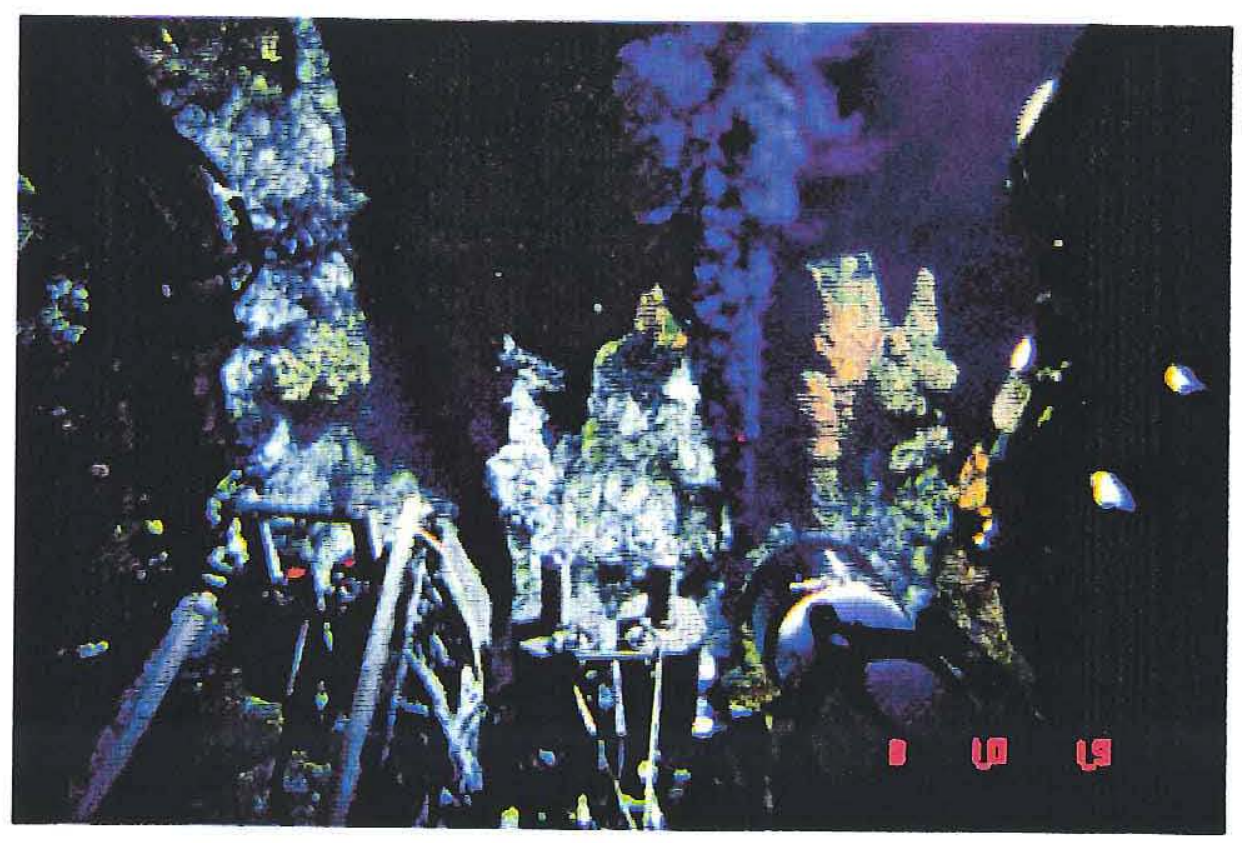

Figure 3.9 


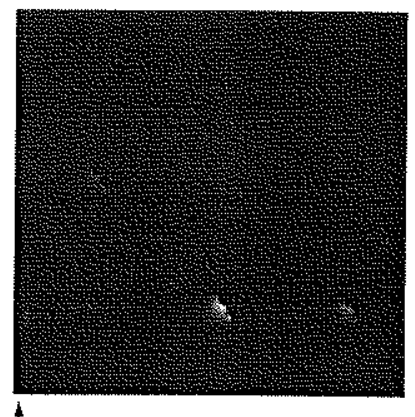

Raw Ambient Light Image:

5 minute exposure

shutter open

20 extra columns read

overscan strip $=$ first 20 columns

Subtract bias image

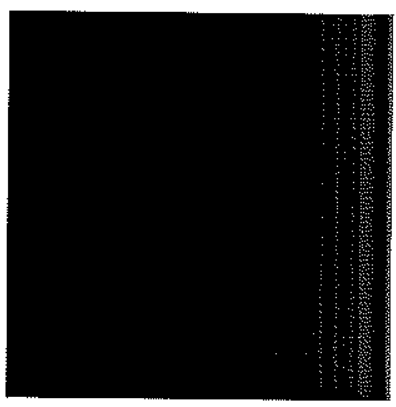

Bias Image:

0 second exposure

shutter closed

Subtract dark image

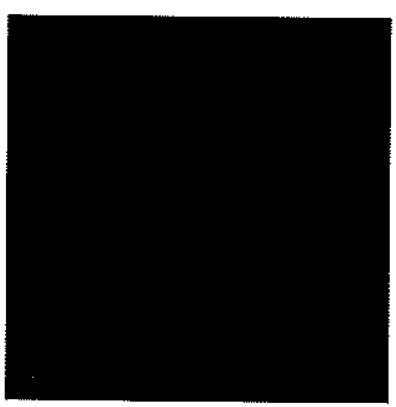

Dark Image:

5 minute exposure shutter closed

Divide by flat field

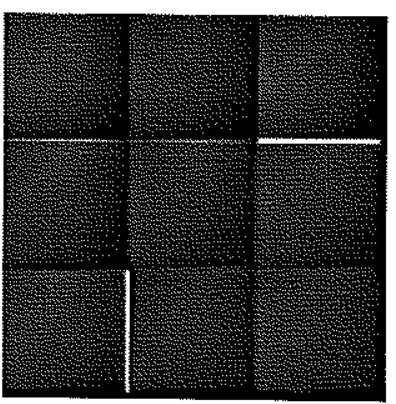

Flat Field Image:

short exposure

shutter open

evenly illuminated

surface

Figure 3.10 


\section{Chapter 4 - Physical Properties of Sea Water \& Vent Fluid}

In analyzing light emission from deep-sea vents, it is important to know the physical properties of both the vent fluids and the surrounding seawater. Many researchers have studied the physical properties of various types of fluids in the lab - water, seawater of varying salinity, etc. However, most of this work has been done at room temperatures and pressures. It is critical to know what those properties are at the seafloor under conditions of extreme temperature and pressure. Some properties are easily measured in situ, but others prove to be more difficult.

Important properties to consider are temperature of both seawater and vent fluid, the chemistry and emissivity of vent fluid, and the attenuation of light in seawater. Temperature can be measured in situ with relative ease. Chemical composition is determined by collecting vent fluid samples and bringing them to the surface, where temperature and pressure conditions are quite different, for analysis. Some attempts have been made to determine emissivity and attenuation in vent environments, but they have been unsuccessful in fully characterizing these properties, which may vary with wavelength.

\subsection{Temperature and Chemistry}

\subsubsection{TEMPERATURE}

The temperature and chemistry of seawater are fairly well characterized and both properties vary with depth and geographical location [Fegley, 1995]. The temperature of the surface ocean can vary by $\sim 30^{\circ} \mathrm{C}$ [Tomczak and Godfrey, 1994] due to ocean currents, season of the year, and global location. The deep ocean, however, has a nearly constant ambient temperature of $\sim 2^{\circ} \mathrm{C}$. 
Hydrothermal venting on mid-ocean ridges can be manifest as low-temperature $\left(<20^{\circ} \mathrm{C}\right)$ diffuse flow [e.g., Edmond et al., 1979], high-temperature (as high as $\left.400^{\circ} \mathrm{C}\right)$ focused flow [e.g., Von Damm et al., 1995a], or anywhere in between. Both hightemperature and low-temperature fluids exiting the seafloor are assumed to derive from a high-temperature end-member fluid. Thus, the low-temperature fluids undergo significant mixing with ambient seawater in the subsurface, while high-temperature fluids do not.

Temperature can be measured in situ rather accurately with the Alvin temperature probes (Table 1). As mentioned in Chapter 2, there is some difficulty in obtaining exact temperature measurements at black smoker vents. This is due both to the sharp thermal gradients caused by rapid mixing with ambient seawater, and disruption of the flow by the temperature probe tip which is $\sim 0.5 \mathrm{~cm}$ in diameter. By contrast, very steady temperature measurements can be made at flange pools due to the relative stability of the fluid and lack of mixing with ambient seawater. Examples of temperature records from a black smoker and a flange pool are shown in Figure 4.1. The flange pool record suggests a time constant of $\sim 15$ seconds for the Alvin high-temperature (high-T) probe.

Only high-temperature vents were imaged with the ALISS camera since only they would be expected to emit significant thermal radiation [Pelli and Chamberlain, 1989]. The imaged $9^{\circ} \mathrm{N}$ EPR vents were measured to be between $310^{\circ}$ and $377^{\circ} \mathrm{C}$. On the Endeavour Segment of the Juan de Fuca Ridge, the imaged black smokers were $\sim 372^{\circ} \mathrm{C}$, while the flange pools measured a stable $332^{\circ} \mathrm{C}$. However, it should be noted that the portions of the $372^{\circ} \mathrm{C}$ black smoker plumes imaged by ALISS had a temperature of $<372^{\circ} \mathrm{C}$ due to entrainment of ambient seawater. Careful measurements made with the Alvin high-T probe just outside of the orifice of Puffer vent (in the Main Endeavour Field) on dive 3234 revealed a temperature of $\sim 330^{\circ} \mathrm{C}$ (Figure 4.1) while measurements at Sully vent on dive 3238 recorded $\sim 340^{\circ} \mathrm{C}$. The lower temperature at Puffer vent may be due to the fact that the 
exiting hydrothermal fluid is directed downward from the sulfide structure (rather than out of the top) and then rises buoyantly. This would increase entrainment of seawater and associated cooling.

\subsubsection{VENT CheMISTRY}

An overview of ocean properties and composition is given by Fegley [1995]. The chemical composition of seawater is dominated by $\mathrm{Cl}^{-}, \mathrm{Na}^{+}, \mathrm{SO}_{4}^{2-}, \mathrm{Mg}^{2+}, \mathrm{Ca}^{2+}$, and $\mathrm{K}^{+}$. The salinity of the ocean varies from $\sim 33$ to 38 parts per thousand (by mass); however, the relative concentrations of the major elements remain rather constant. Variations in salinity are due primarily to the addition and subtraction of fresh water to the oceans, either via precipitation or river run off.

Hydrothermal fluid that exits the seafloor is simply seawater that has been altered by interaction with oceanic crust [Edmond et al., 1979; Alt, 1995, and references therein]. Hydrothermal circulation within the crust cannot be directly observed, and is difficult to accurately model due to uncertainties in porosity and fluid velocity. However, altered rocks dredged from the seafloor, ophiolites, seafloor drilling, and fluid chemistry can help constrain the processes that are occurring in the subsurface. Seawater seeps into porous crust at mid-ocean ridges, and travels downward where it is heated by a magma source and reacts with the surrounding rock. At high temperatures $\left(>350^{\circ} \mathrm{C}\right)$, the fluid becomes buoyant and rises rapidly to the surface [Alt, 1995]. The major chemical changes include loss of $\mathrm{Mg}^{2+}$ and sulfate $\left(\mathrm{SO}_{4}\right)^{2-}$, and increases in $\mathrm{Ca}$, Si, alkali metals ( $\mathrm{Li}, \mathrm{K}, \mathrm{Rb}, \mathrm{Cs}$ ), and trace metals $(\mathrm{Cu}, \mathrm{Fe}, \mathrm{Zn}, \mathrm{Mn})$. Hydrothermal fluids are also often high in gases such as $\mathrm{H}_{2} \mathrm{~S}$ and $\mathrm{CO}_{2}$. The concentration of some elements, such as $\mathrm{Cl}$ and $\mathrm{Na}$, can fall either above or below seawater values due to processes such as phase separation, and variation in water-rock ratios [Von Damm, 1995b]. 
In situ determination of fluid chemistry is an emerging field. Currently, samples are collected in titanium bottles and returned to shore for analysis [see Von Damm, 1983, for a description of the sample bottles and analyses]. In young vent systems where magma emplacement is occurring or cracking provides new pathways into hot rock, chemistry can change rapidly over time [Von Damm et al., 1995a]. However, in mature vent systems, the chemistry can remain quite stable for years [Campbell et al., 1988]. For example, the Venture Hydrothermal Field on the East Pacific Rise is a young hydrothermal system. The venting occurring there was redistributed by a large eruptive event in 1991 [Haymon et al., 1993]. This eruption and subsequent small eruptions can be seen in the chemical record as drops in $\mathrm{Cl}$ (and related element) concentration which subsequently increase towards (and in some cases exceed) seawater values [Oosting and Von Damm, 1996; Von Damm et al., 1995a]. In contrast, the Main Endeavour Field (MEF) on the Juan de Fuca Ridge is a relatively mature system, and thus vent chemistry has remained relatively stable for years. A chemical gradient can be seen between the northeastern vents and the southwestern vents [Butterfield et al., 1994]. The northeastern vents show chlorinities as high as $94 \%$ of seawater chlorinity, while the southwestern vents have chlorinities as low as $47 \%$ of seawater chlorinity (Table 4.2). In addition, the relative shallowness of the MEF ( 2200 m) allows boiling to occur at or just below the seafloor [Bischoff and Pitzer, 1985].

Water samples were collected at vents imaged by ALISS on the Endeavour Segment of the Juan de Fuca Ridge, and at $9^{\circ} \mathrm{N}$ on the East Pacific Rise. The samples were analyzed by K. Von Damm at the University of New Hampshire, M. Lilley at the University of Washington, and D. Butterfield at NOAA, Pacific Marine Environmental Laboratory. Chemical data from the imaged vents is given in Table 4.3 and Table 4.4. 


\subsection{Attenuation}

Unlike astronomers, who observe faint stars across vast distances containing various atmospheric and deep-space conditions, we have the advantage of observing our object (vents) through a mere $50 \mathrm{~cm}$ of sea water. However, since the light emitted at hydrothermal vents travels through $50 \mathrm{~cm}$ of water before reaching the ALISS camera, the attenuation of light in water is an important optical property that must be known when analyzing data. Attenuation is the decrease in the intensity of light as it passes through a medium resulting from both absorption and scattering, as described by the equations

$$
\begin{gathered}
I_{x}=I_{o} \Omega_{x} e^{-c(\lambda) \cdot x} \\
c(\lambda)=a(\lambda)+b(\lambda)
\end{gathered}
$$

where $I_{o}$ is the intensity at the source, $I_{x}$ is the intensity at some distance $x, \Omega_{x}$ is the solid angle subtended by the detector at distance $x$ (which accounts for decreased intensity due to spherical spreading), $c$ is the attenuation coefficient as a function of wavelength $(\lambda)$, and $a$ and $b$ are the absorption and total scattering coefficients as a function of wavelength, respectively. (It should be noted that decreasing intensity due to geometric spreading is a process distinct from attenuation). It is the wavelength dependence of attenuation that confines much of ocean optical research to the visible region of the spectrum where light is attenuated the least (Figure 4.2).

A number of researchers have experimentally determined the attenuation and absorption coefficients of both pure water [e.g., Collins, 1925; Curcio and Petty, 1951; Sullivan, 1963; Hale and Querry, 1973; Kou et al., 1993; Pope and Fry, 1997] and seawater [e.g., Smith and Baker, 1981]. In most cases, the water used in these experiments is filtered to remove particulate matter which causes scattering. Since scattering due to the water itself is very small, attenuation measured in those cases is 
primarily due to an absorbance effect [Jerlov, 1968]. A summary of the absorption and attenuation coefficients found in the literature are shown in Figure 4.3. A number of shoulders and peaks are present in the visible and near infrared regions of the absorption spectrum at the overtones of the $\mathrm{O}-\mathrm{H}$ vibrational frequencies. The wavelengths corresponding to those frequencies are: $3049 \mathrm{~nm}$ (symmetric stretch), $6079 \mathrm{~nm}$ (bend), and $2865 \mathrm{~nm}$ (asymmetric stretch) [Bayly et al., 1963; Pegau et al., 1997].

Collins [1925] was the first to investigate the effect of temperature on the absorbance spectrum of water. He found that as the temperature is decreased from $95^{\circ} \mathrm{C}$ to $0^{\circ} \mathrm{C}$, peaks in the spectrum at the overtones of the $\mathrm{O}-\mathrm{H}$ vibrational frequencies shift to longer wavelengths and become less pronounced. Pegau et al. [1997] also found that changes in temperature primarily affect wavelengths associated with overtone frequencies. Collins [1925] attributes the change in absorbance with temperature to the change in the components of water. Liquid water contains individual water molecules $\left(\mathrm{H}_{2} \mathrm{O}\right)$, but also various combination of water molecules $\left(\mathrm{H}_{2} \mathrm{O}\right)_{n}$ formed by hydrogen bonding [Collins, 1925; Dera, 1992]. The lower the temperature the larger the number of combined molecules. The absorption spectrum of ice, in which all of the molecules are bonded together, differs from that of liquid water, in which there are some combined molecules, and from vapor, which presumably has no combined molecules. The absorption peaks shift to longer wavelengths and become less pronounced in the $400-1000 \mathrm{~nm}$ region as the number of combined molecules increases [Collins, 1925].

The attenuation and absorption coefficients found in the literature (Figure 4.3) are not directly appropriate for use in the ALISS calculations for a number of reasons. First of all, most of the literature values are for pure (non-saline) water. Pegau et al. [1997] showed that absorption is affected by salinity. Changes in the attenuation coefficient due to a $35 \%$ change in salinity are very small with a maximum effect of only $\sim 1 \%$ at $750 \mathrm{~nm}$. 
Thus, the salinity effect is negligible. Secondly, and most importantly, is the fact that the experiments described in the literature use filtered water to remove the effects of scattering. In the vent environment, scattering can be very important. Particulate matter is produced by precipitation in hydrothermal plumes. In fact, when searching for a vent field during an Alvin dive, an increase in particles in the water is used to infer proximity to an active vent. The amount and composition of the particles in the vent environment are dependent upon the fluid flux and chemistry of the local vents. Thus, scattering effects can vary from site to site, just as chemistry and flux vary from vent to vent. Thirdly, the ambient temperature at the ocean floor is $\sim 2^{\circ} \mathrm{C}$ - much colder than the $20^{\circ} \mathrm{C}$ water used in most laboratory measurements. This temperature difference is important, as noted above. Likewise, it is possible that pressure as well as temperature can affect the attenuation in water. The hydrothermal vents imaged by ALISS are located on mid-ocean ridges at pressures of $\sim 220$-250 bar, much higher than the atmospheric pressure ( $\sim$ bar) present in a laboratory setting. However, no data exists on the effect of pressure on the absorbance of water in a vent environment.

\subsection{IN SITU ATtENUATION MEASUREMENTS}

Since it is important to know the attenuation coefficients for the deep-sea hydrothermal environments where ALISS data were collected, a series of experiments were performed using a light source and the OPUS (Optical Properties Underwater Sensor) instrument [White et al., 1996b]. These experiments were conducted on two cruises to the $9-10^{\circ} \mathrm{N}$ area of the EPR. Measurements were made of the light source at two distances with all of Alvin's external lights extinguished. The distances varied from as short at 14 $\mathrm{cm}$ to as long as $58.5 \mathrm{~cm}$. Both the light source and the OPUS instrument were mounted on a fiberglass track to ensure proper alignment and exact distances. By measuring the 
intensity of the light source at two distances, the attenuation coefficient could be determined by the equation

$$
c=\frac{1}{z_{2}-z_{1}} \cdot \ln \left(\frac{I_{1}}{I_{2}} \frac{\Omega_{2}}{\Omega_{1}}\right)
$$

where $c$ is the attenuation coefficient, $I_{l}$ is the intensity of the light source and $\Omega_{1}$ is the solid angle at distance $z_{I}$, and $I_{2}$ and $\Omega_{2}$ are the intensity and solid angle, respectively, at distance $z_{2}$. The results of the OPUS attenuation experiments are show in Figure 4.3. The OPUS data show higher attenuation coefficients at wavelengths $<700 \mathrm{~nm}$ than literature values for pure water. This is probably due to the scattering effect of particles in the water. The peaks and shoulders seen in the literature are not as pronounced, or are not present in the OPUS data. This is mostly likely due to a combination of factors. The OPUS filters are $100 \mathrm{~nm}$ in bandwidth, much coarser than the laboratory measurements made every 10 $\mathrm{nm}$. Thus, fine scale features, such as peaks and shoulders $50 \mathrm{~nm}$ wide, cannot be resolved by the OPUS instrument. Secondly, the low ambient temperatures in the vent environments would tend to flatten the features at vibrational overtones, as discussed above. Despite these factors, the OPUS values at 850,900 , and 950 are inexplicably low. That is, physically one would not expect to measure an attenuation coefficient of seawater that is less than (by a factor of $\sim 2$ ) the absorption coefficient of pure water.

It should also be noted that it is difficult to measure the attenuation in the visible region due to the low absorption in that region. Ideally, path lengths on the order of 1 meter and greater would be used. Due to the limited space on the Alvin science basket and the difficulty in positioning and aligning instruments that far apart on the sea floor, path lengths on that order were not feasible. However, due to the small attenuation coefficients in the visible (on the order of $10^{-3}$ to $10^{-4}$ ), variation in the attenuation values have little effect on inferences drawn from data at a distance of $50 \mathrm{~cm}$. 
Due to the inexplicably low attenuation coefficients above $800 \mathrm{~nm}$, and the coarseness of the attenuation data, the OPUS values were not used for analysis of the ALISS data. Instead, I have come up with an attenuation curve, derived from literature values, which takes into consideration salinity and temperature effects. Rather than averaging the data to create a composite curve, I selected the most recent data, giving preference to experiments using actual seawater. At wavelengths less than $700 \mathrm{~nm}, \mathrm{I}$ use the data from Smith and Baker [1981] for seawater. Given the amount of particles in the vent environment, the actual attenuation is probably higher than that from Smith and Baker [1981] in the visible range. Thus, the light observed in this region is underestimated. However, given the low coefficient values $\left(10^{-3}\right.$ to $\left.10^{-4}\right)$ and the distance through which ALISS is imaging $(50 \mathrm{~cm})$, this has very little effect — doubling the attenuation coefficient from $2 \times 10^{-3}$ to $4 \times 10^{-3}$ produces only a $1 \%$ change in the data. For wavelengths from 700 to $1000 \mathrm{~nm}$, the measurements of Kou et al. [1993] are used. These measurements are for pure water at $22^{\circ} \mathrm{C}$. Pegau et al. [1997] show how salinity and temperature affect attenuation. In fact, salinity has little effect and temperature primarily affects attenuation at $975 \mathrm{~nm}$. Therefore, the values of Kou et al. [1993] were modified only at $975 \mathrm{~nm}$ to account for the decrease in attenuation due to the ambient water being $2^{\circ} \mathrm{C}$ rather than $22^{\circ} \mathrm{C}$. The values used in the ALISS calculations are shown by the solid line in Figure 4.3.

\subsubsection{SCATTERING}

As stated above, attenuation is due to both absorption and scattering. Scattering is the random change in the direction of light rays caused by the presence of inhomogeneities in a medium. The scattering process is made up of three phenomena: diffraction, refraction, and reflection [Kullenberg, 1974]. Scattering can be caused by inhomogeneities that are much smaller than the wavelengths of visible light — called molecular or Rayleigh 
scattering, or by particles the same size or larger than the wavelengths of light - called Mie scattering.

Rayleigh scattering occurs in the atmosphere and in pure water. The intensity distribution of light scattered by a particle much smaller than the wavelength of light is given by:

$$
I(\theta)=\frac{I_{0}}{d^{2}} \cdot \frac{8 \pi^{4} p^{2}}{\lambda^{4}}\left(1+\cos ^{2} \theta\right)
$$

where $I(\theta)$ is the intensity at the observer a distance $d$ away and at angle $\theta$ from the incident ray, $p$ is the polarizability of the particle, and $\lambda$ is the wavelength of the light [Morel, 1974]. The scattering at $0^{\circ}$ and $180^{\circ}$ is twice the scattering at $90^{\circ}$ and $270^{\circ}$. The $1 / \lambda^{4}$ dependence leads to selective scattering where shorter wavelengths (i.e., blue light) are scattered more than longer wavelengths. It is Rayleigh scattering in the atmosphere which causes the sky to look blue.

Much of the particulate matter in the ocean is too large to produce Rayleigh scattering. The Mie theory describes scattering from homogenous spheres immersed in a uniform medium of different refractive index, and is applicable to scattering by marine particles [Dera, 1992]. A rigorous mathematical look at Mie scattering is given by Van de Hulst [1957]. The main characteristics of Mie scattering are strong forward scattering through small angles, a scattering minimum at $\sim 130^{\circ}$ (rather than $90^{\circ}$ for Rayleigh scattering), and a decrease in selectivity as the particle size increases (i.e., all wavelengths are scattered to a similar degree) [Dera, 1992].

The seawater attenuation measurements described in the previous section determine attenuation due to the effects of both absorption and scattering. The scattering component of seawater need not be individually determined. However, another place where scattering must be considered is within the plume itself. It is the large number of particles 
precipitating in a plume that gives it a smoky appearance. Attenuation in turbid coastal waters can be $\sim 5$ times higher than in clear ocean waters at $500 \mathrm{~nm}$ due to scattering effects [Wolfe and Zissis, 1989]. The particles that precipitate in a hydrothermal plume are probably similar in size to particles in coastal areas, and thus these particles will cause Mie scattering that can significantly affect attenuation within the plume. This scattering is an important effect and will be considered in the following section on determining the emissivity of a plume.

\subsection{Emissivitry}

Emissivity (as mentioned in Chapter 2) is the ratio of energy emitted by an object to that emitted by an ideal black body at the same temperature:

$$
E(\lambda, t)=J(\lambda, t) / J_{b}(\lambda, t)
$$

where $E$ is the emissivity at a given wavelength $(\lambda)$ and temperature $(t)$, and $J$ and $J_{b}$ are the spectral emissive powers of a body and a black body, respectively. Thus, an ideal black body has an emissivity of one, and gray bodies have emissivities less than one. The emissivity and absorptivity of any thermal radiator are equal for a given temperature [Finkelnburg, 1949]. Therefore, an opaque object will have a higher emissivity than a semi-transparent object. Also, just as absorbance varies with wavelength (e.g., Figure 4.3) so, too, may emissivity.

Consider radiation incident on a layer of material (Figure 4.4A). The incident radiation $\left(J_{i}\right)$ can be either reflected $\left(J_{r}\right)$, transmitted $\left(J_{t}\right)$, or absorbed by the layer $\left(J_{a}\right)$. An opaque body has a transmissivity of zero. The transmissivity $(T)$ of a non-opaque layer is given by its absorption coefficient $(a)$ and the thickness of the layer $(d)$ :

$$
T(\lambda, t)=\frac{J_{t}(\lambda, t)}{J_{e}(\lambda, t)}=e^{-a(\lambda, t) \cdot d}
$$


where $J_{e}(\lambda, t)$ is the radiation entering the layer (i.e., $J_{i}-J_{r}$ ). Thus, the emission from a semi-transparent body approaches that of an opaque body with increasing thickness [Finkelnburg, 1949; Gardon, 1956].

Kirchoff's Law relates emissivity to reflectivity $(R)$ for an opaque body $(T=0)$ :

$$
E(\lambda, t)=1-R(\lambda, t)
$$

McMahon [1950] extends Kirchoff's Law to partially transparent bodies with radiation normal to plane parallel surfaces.

$$
E(\lambda, t)=\frac{\{1-R(\lambda, t)\}\{1-T(\lambda, t)\}}{\{1-R(\lambda, t) \cdot T(\lambda, t)\}}
$$

He also calculates the apparent reflectivity $\left(R^{*}\right)$ and apparent transmissivity $\left(T^{*}\right)$ - which include the effects of internal reflections - and shows that:

$$
E(\lambda, t)+R^{*}(\lambda, t)+T^{*}(\lambda, t)=1
$$

Thus, by measuring the apparent reflectivity and apparent transmissivity, we can determine the emissivity (Figure 4.4A). However, unlike McMahon's plane parallel slab, black smoker plumes are three dimensional structures better approximated by a cylinder. In addition, McMahon does not consider the effects of scattering and diffuse reflection [Nicodemus, 1967]. Given the amount of particulate matter in black smoker plumes, scattering is an important effect to consider.

The emissivity of hydrothermal fluid has not yet been measured. In Chapter 5, emissivity is estimated from the ALISS data. However, this estimation cannot characterize wavelength dependence. Because emissivity probably varies with wavelength, detailed experiments must be performed on future cruises to determine the emissivity of hydrothermal fluid in the $400-1000 \mathrm{~nm}$ region of the spectrum.

One way in which emissivity of a black smoker plume could be determined is by illuminating the plume with a collimated light source (i.e., a laser) and making a number of 
measurements around the plume (Figure 4.4B). The light source used would need to be much brighter than the ambient vent emission, such that the ambient emission is an insignificant factor and can be neglected. If the light spot is much smaller than the radius of the vent, then we have a case similar to McMahon's [1950] plane-parallel approximation but with an additional scattering component. The scattering caused by plume particles is most likely Mie scattering (due to the particle size), which results in scattering at all wavelengths and strong forward scattering. The emissivity is then given by:

$$
E(\lambda, t)=1-R^{*}(\lambda, t)-T^{*}(\lambda, t)-S *(\lambda, t)
$$

where $S^{*}$ is the percent of incident radiation scattered at angles greater than $0^{\circ}$ and less than $180^{\circ}$ from the light source. However, scattering in the plume is not confined to a plane. Therefore, some of the scattered radiation may exit the plume above or below the plane of incident radiation; or it may be scattered up or down the plume. This radiation cannot be constrained, causing the scattered radiation to be underestimated and the emissivity to be overestimated.

To account for some of this "lost" radiation, consider a sphere with a diameter equal to that of the plume (Figure 4.5). The amount of radiation exiting the plume (due to the incident radiation) is estimated by making a series of measurements around the plume $(0-$ $180^{\circ}$ ) and then summing this radiation both around the plume in the horizontal plane and about the axis made by the incident beam (i.e., inscribing a sphere within the plume). Thus, the emissivity is given by:

$$
E(\lambda, t)=1-\left[\frac{\int_{0}^{2 \pi} \int_{0}^{2 \pi} J_{s} d \theta d \phi}{J_{i}}\right]
$$

where $J_{s}$ is all of the exiting radiation (from transmission, reflection, and scattering), $\theta$ is the angle about the $\mathrm{z}$-axis, and $\phi$ is the angle about the $y$-axis (Figure 4.5). As noted, 
particles the same size or larger than the wavelength of light will primarily scatter light forward. Thus, it is possible that the amount of light scattered away from the incident direction will be negligible. Only actual measurements can ascertain the importance of the various components of scattered light.

An estimate of emissivity and how it varies with wavelength can be determined with the ALISS camera, a laser light source, and the process described above. Because of the size of the ALISS camera, it must remained fixed in the Alvin science basket while the laser is moved around the plume from $0^{\circ}$ (at the ALISS camera) to $180^{\circ}$. By taking ALISS images at a number of angles from the laser, the distribution of scattered light can be determined. Images at $0^{\circ}$ and $180^{\circ}$ will determine the reflectivity and transmissivity of the plume, respectively. Ambient light images should also be taken. While the laser should be much greater in intensity than the ambient vent emission, it is possible that only a small amount of light will be scattered at some angles. Thus, the ambient emission may be a significant factor at those angles. Also, due to the narrow bandwidth of lasers, at least two to three lasers of different wavelengths should be used to determine the dependence of emissivity on wavelength. The emissivity is then simply one minus the scattered light over the incident light at various wavelengths (Equation 4.11).

Emissivity is an important factor in determining thermal radiation. As will be discussed in the following chapter, thermal radiation is the dominant component of vent light. However, in order to accurately predict thermal radiation, the emissivity and how it varies with wavelength must be well known. Only after thermal radiation can be well characterized can the contribution of other non-thermal sources be carefully determined. 
Table 4.1 - Alvin temperature probes

\begin{tabular}{|l|c|c|c|}
\hline & Range & Accuracy & Resolution \\
\hline Low temp. probe & $0-20^{\circ} \mathrm{C}$ & $0.5 \mathrm{deg}$. & $0.001 \mathrm{deg}$. \\
\hline High temp. probe & $30-800^{\circ} \mathrm{C}$ & $5 \mathrm{deg}$. & $0.1 \mathrm{deg}$. \\
\hline
\end{tabular}

Table 4.2 - Endeavour Vent Chemistry (1994)

\begin{tabular}{|l|c|c|c|c|c|c|}
\hline Vent & $\begin{array}{c}\text { Temp. } \\
\left({ }^{\circ} \mathbf{C}\right)\end{array}$ & $\begin{array}{c}\mathbf{C l} \\
(\mathbf{m m o l} / \mathbf{k g})\end{array}$ & $\begin{array}{c}\mathbf{N a} \\
(\mathbf{m m o l} / \mathbf{k g})\end{array}$ & $\begin{array}{c}\mathbf{K} \\
(\mathbf{m m o l} / \mathbf{k g})\end{array}$ & $\begin{array}{c}\mathbf{H}_{2} \mathbf{S} \\
(\mathbf{m m o l} / \mathbf{k g})\end{array}$ & $\begin{array}{c}\mathbf{C O}_{2} \\
(\mathbf{m m o l} / \mathbf{k g})\end{array}$ \\
\hline \hline Lobo & 346 & $428 \pm 4$ & $336 \pm 6$ & $24.5 \pm 0.6$ & $5.0 \pm 0.8$ & - \\
\hline Dudley & - & $349 \pm 5$ & $271 \pm 7$ & $19.9 \pm 0.5$ & $3.0 \pm 0.5$ & - \\
\hline Peanut & 350 & $253 \pm 30$ & $216 \pm 30$ & $13.5 \pm 2.5$ & $8.1 \pm 1.3$ & - \\
\hline
\end{tabular}

[All data from Butterfield et al., 1994]

Table 4.3 - Endeavour Vent Chemistry (1998)

\begin{tabular}{|l|c|c|c|c|c|c|}
\hline Vent & $\begin{array}{c}\text { Temp. } \\
\left({ }^{\circ} \mathbf{C}\right)\end{array}$ & $\begin{array}{c}\mathbf{C l}^{\mathbf{a}} \\
(\mathbf{m m o l} / \mathbf{k g})\end{array}$ & $\begin{array}{c}\mathbf{N a}^{\mathbf{a}} \\
(\mathbf{m m o l} / \mathbf{k g})\end{array}$ & $\begin{array}{c}\mathbf{K}^{\mathbf{a}} \\
(\mathbf{m m o l} / \mathbf{k g})\end{array}$ & $\begin{array}{c}\mathbf{H}_{2} \mathbf{S}^{\mathbf{b}} \\
(\mathbf{m m o l} / \mathbf{k g})\end{array}$ & $\begin{array}{c}\mathbf{C O}_{\mathbf{2}}{ }^{\mathbf{b}} \\
(\mathbf{m m o l} / \mathbf{k g})\end{array}$ \\
\hline \hline Puffer & 372 & $\sim 126$ & $\sim 100$ & $\sim 8$ & 12.5 & 22.8 \\
\hline Sully & 373 & $98-162$ & $\sim 118$ & $\sim 8.9$ & 1.35 & 19.6 \\
\hline
\end{tabular}

${ }^{\mathrm{a}}$ [D. Butterfield, per. comm.]

${ }^{\mathrm{b}}[M$. Lilley, per. comm.]

Table $4.4-9^{\circ} \mathrm{N}$ Vent Chemistry (1997)

\begin{tabular}{|c|c|c|c|c|c|c|}
\hline Vent & $\begin{array}{c}\text { Temp. } \\
\left({ }^{\circ} \mathbf{C}\right)\end{array}$ & $\begin{array}{c}\mathbf{C l}^{\mathbf{a}} \\
(\mathbf{m m o l} / \mathbf{k g})\end{array}$ & $\begin{array}{c}\mathbf{N a}^{\mathbf{a}} \\
(\mathbf{m m o l} / \mathbf{k g})\end{array}$ & $\begin{array}{c}\mathbf{K}^{\mathbf{a}} \\
(\mathbf{m m o l} / \mathbf{k g})\end{array}$ & $\begin{array}{c}\mathbf{H}_{2} \mathbf{S}^{\mathbf{b}} \\
(\mathbf{m m o l} / \mathbf{k g})\end{array}$ & $\begin{array}{c}\mathbf{C O}_{2}{ }^{\mathbf{b}} \\
(\mathbf{m m o l} / \mathbf{k g})\end{array}$ \\
\hline \hline P Vent & 377 & - & $450 \pm 10$ & $17.3 \pm 0.3$ & 6.6 & 85 \\
\hline Q Vent & 341 & $551-564$ & $469-475$ & 16.9 & 4 & 160 \\
\hline $\begin{array}{c}\text { L Vent } \\
\text { (AdV 4-L) }\end{array}$ & 315 & $598-599$ & $475-479$ & $19.7-19.3$ & 7.8 & 12.8 \\
\hline V Vent & 315 & 423 & 378 & 16.9 & - & 29.7 \\
\hline
\end{tabular}

${ }^{\mathrm{a}}$ [K. Von Damm, per. comm.]

${ }^{\mathrm{b}}$ [M. Lilley, per. comm.] 


\section{Figure Captions}

Figure 4.1 Alvin high-temperature probe records from (A) Puffer vent (dive 3234) $372^{\circ} \mathrm{C}$ black smoker, and (B) Lobo flange (dive 3235 ) $-332^{\circ} \mathrm{C}$ flange pool. The steady $330^{\circ} \mathrm{C}$ measurement at Puffer vent was made at the orifice (i.e., the portion of the vent that ALISS images), rather than inside the chimney. The record from Lobo suggests a time constant of $\sim 15$ second for the temperature probe.

Figure 4.2 Absorption coefficient of pure water from the ultraviolet to the infrared. Wavelength is given in $\mu \mathrm{m}$ and increases to the left. The dashed lines indicate the visible region of the spectrum. [From Wolfe and Zissis, 1989]

Figure 4.3 Attenuation coefficients of water and seawater found in the literature. The dark line indicates values used during ALISS processing (taken from Smith and Baker [1981] and Kou et al. [1993], and modified for temperature as described by Pegau et al. [1997]). Solid circles indicate attenuation values measured using the OPUS instrument with $100 \mathrm{~nm}$ bandwidth filters. The OPUS data in the visible region are likely higher than the literature values due to increased scattering in vent environments.

Figure 4.4 A) Optical properties of a slab $d \mathrm{~cm}$ thick with an emissivity of E. Incident radiation $\left(J_{i}\right)$ is either reflected $\left(J_{r}\right)$, transmitted $\left(J_{t}\right)$, or absorbed $\left(J_{a}\right)$ by the layer.

B) Collimated radiation incident on a cylindrical object may be scattered at all angles in a plane. Thus, the total scattered radiation emitted from the body must be considered, as well as reflected and transmitted radiation, when calculating emissivity. 
Figure 4.5 Radiation incident on a vent plume $\left(J_{i}\right)$ will be scattered in all directions $\left(J_{s}\right)$. Thus, the emissivity is the ratio of all of the exiting radiation to the incident radiation. Since the plume can be approximated by a cylinder, radiation scattered up and down cannot be measured. The approximate distribution of the scattered radiation can be determined by measuring around one side of the plume (i.e., $\theta=0^{\circ}$ to $180^{\circ}$ from the light source). The total amount of scattered radiation exiting a sphere inscribed within the plume can then be estimated by summing the radiation around the plume $\left(\theta=0^{\circ}\right.$ to $\left.360^{\circ}\right)$ and about the axis of the incident radiation $\left(\phi=0^{\circ}\right.$ to $\left.360^{\circ}\right)$. 
A

Dive 3234 - Puffer Vent

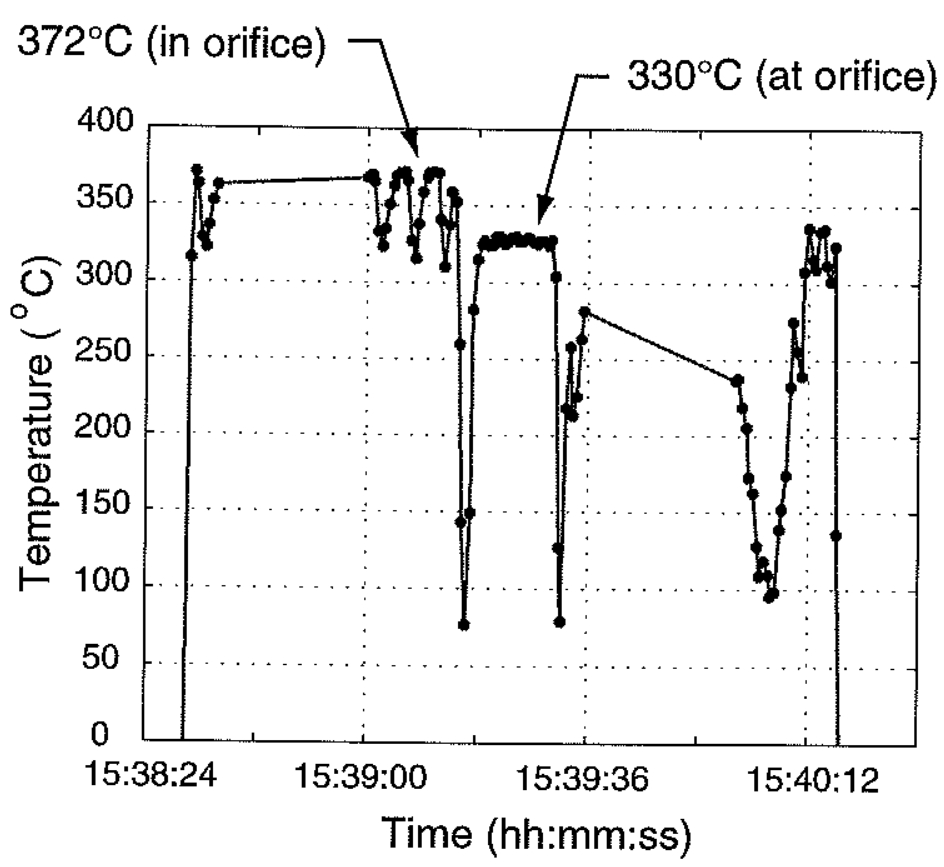

B

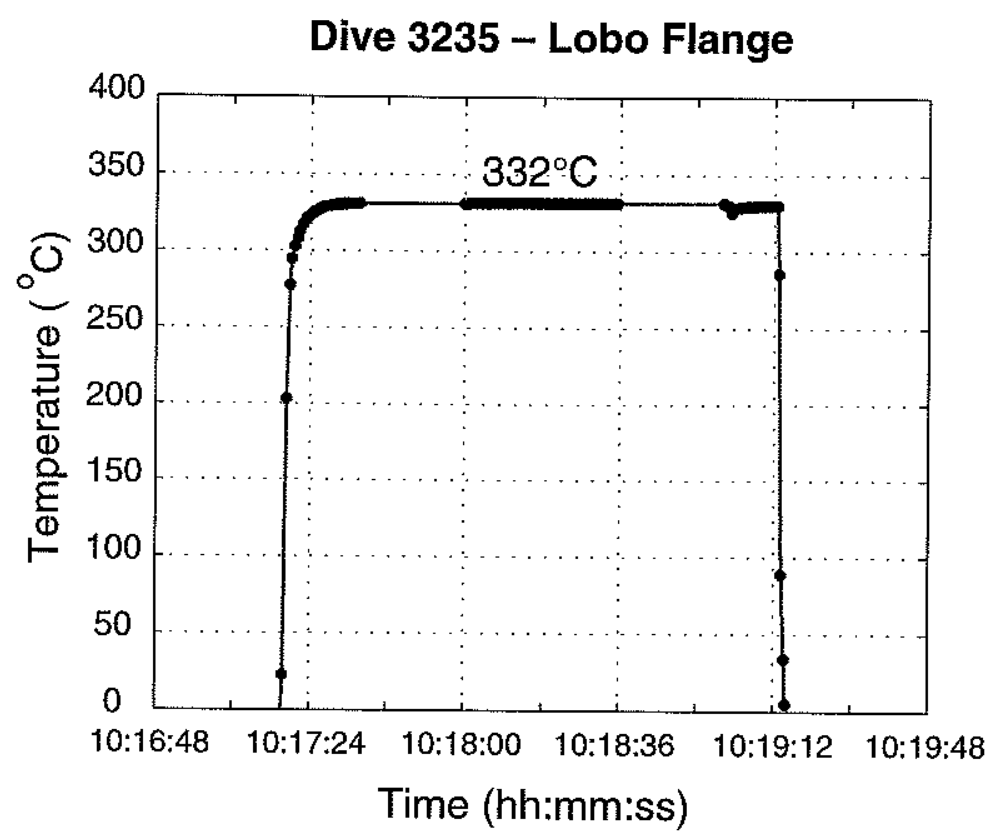

Figure 4.1 


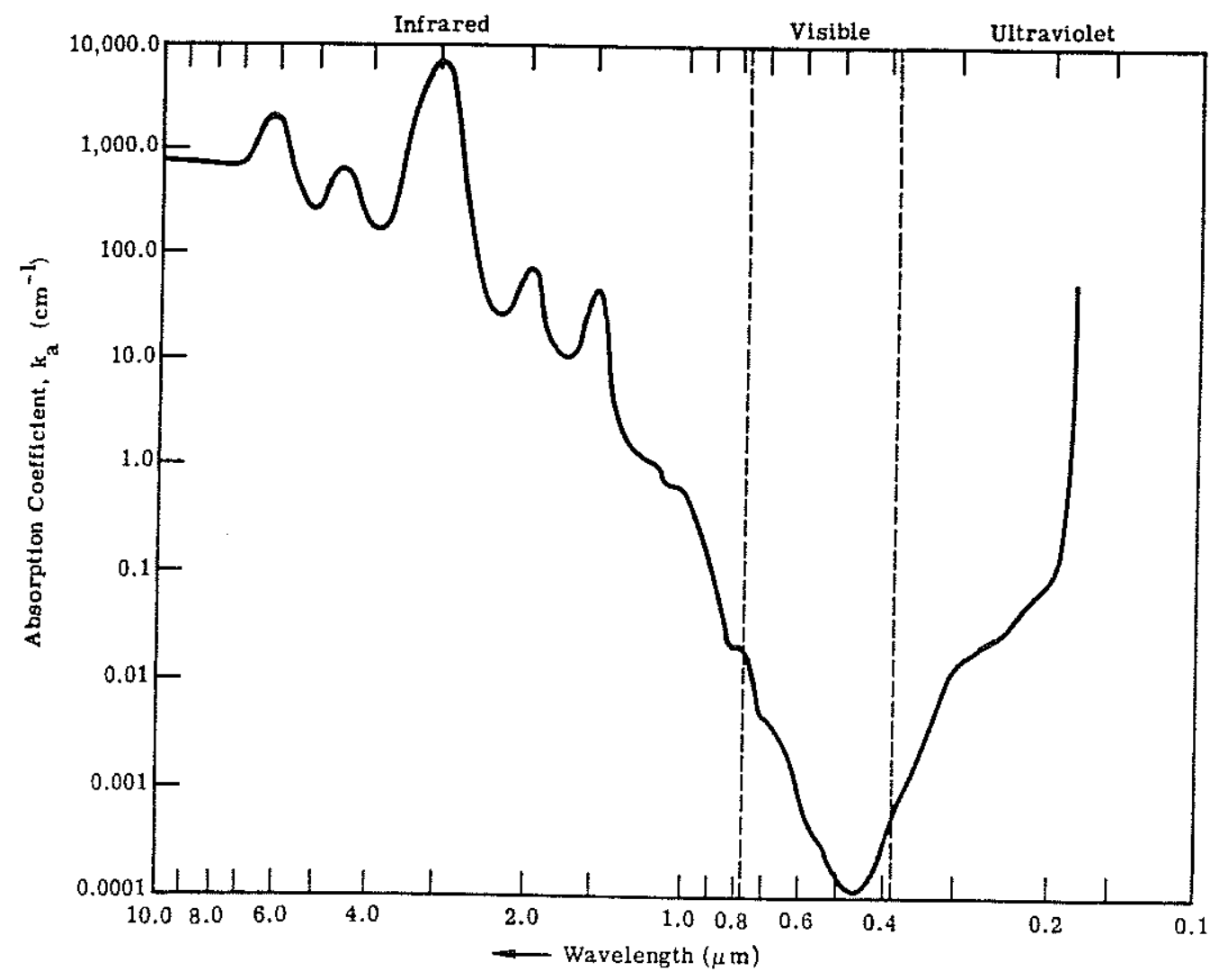

Figure 4.2 


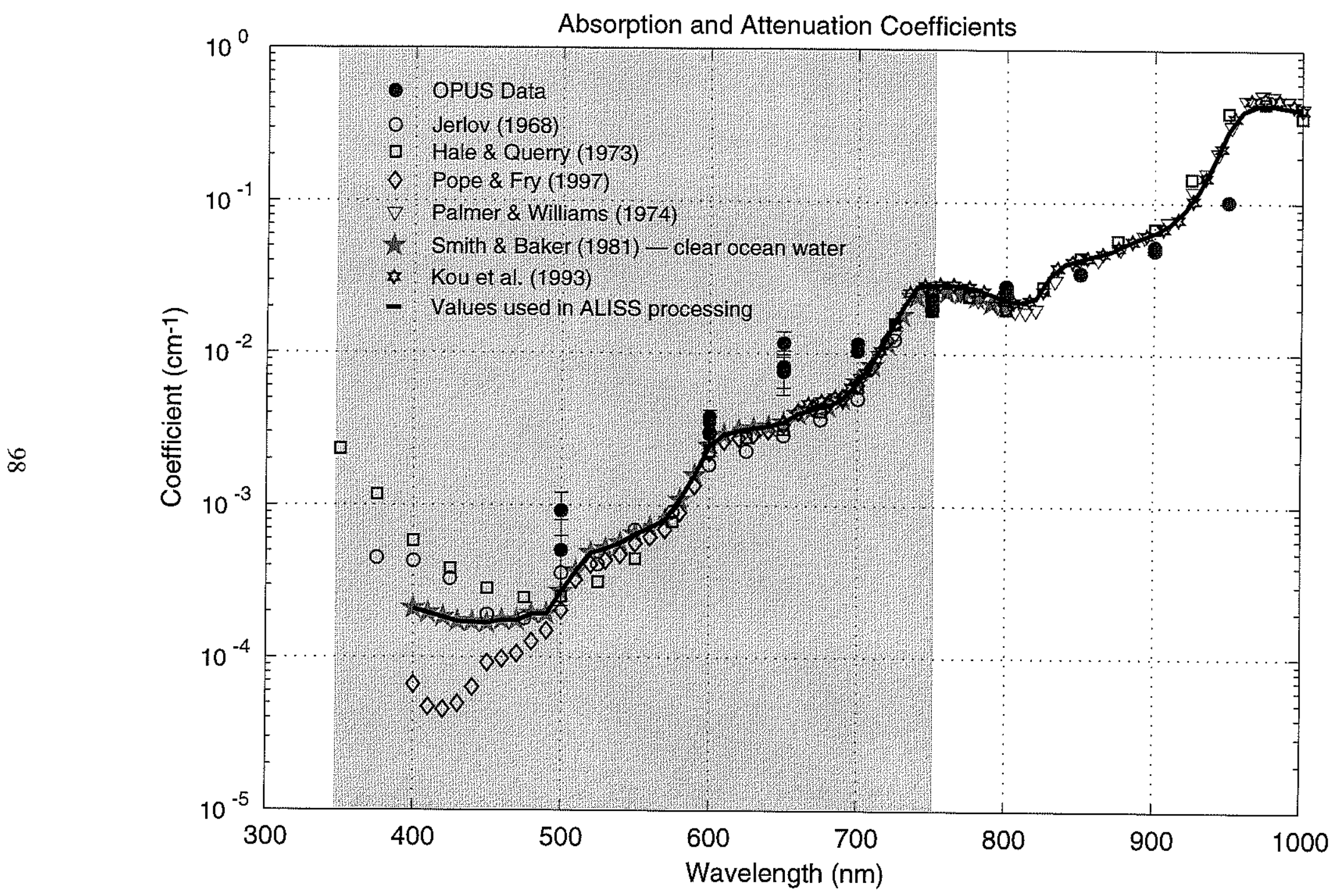

Figure 4.3 


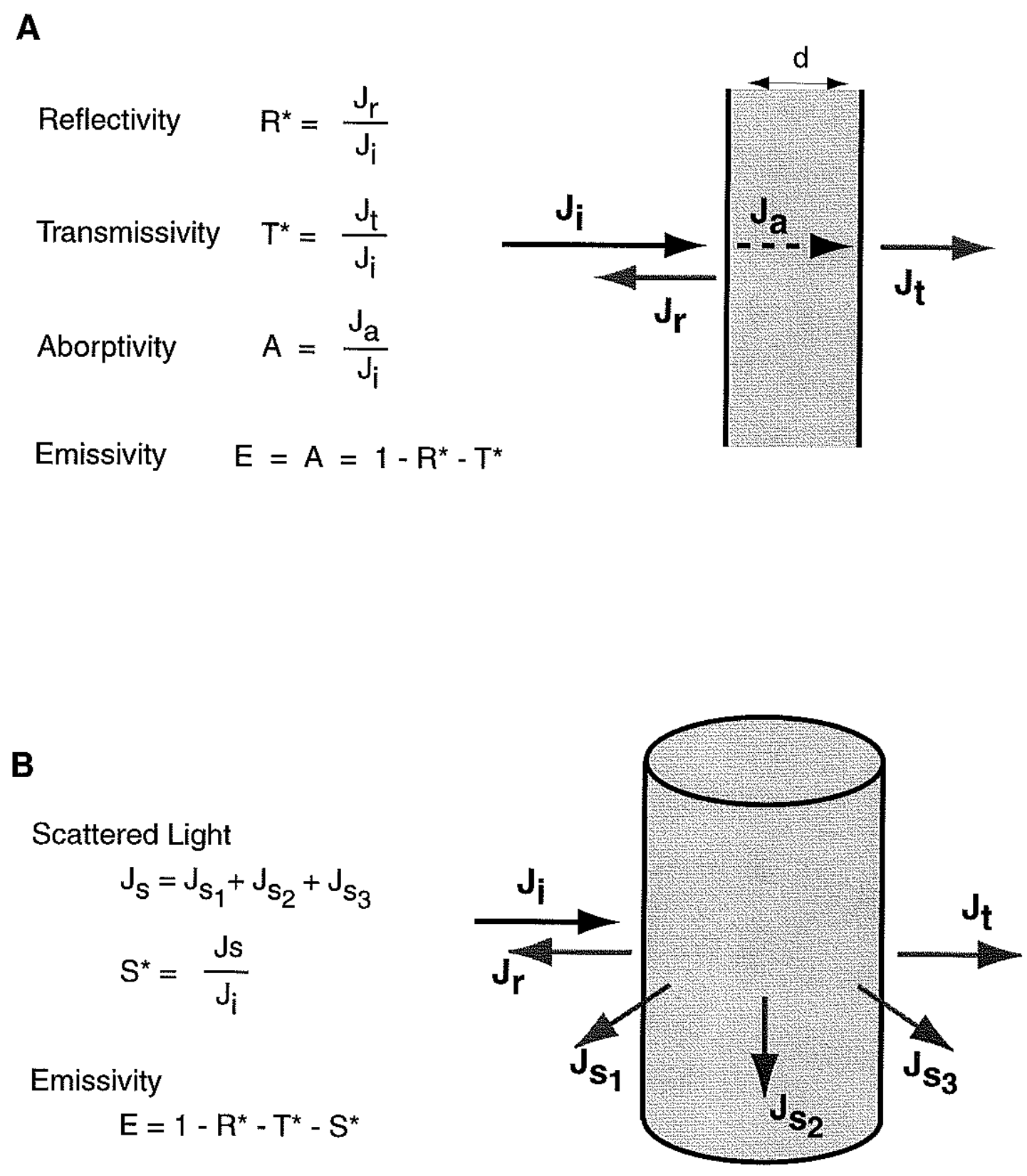

Figure 4.4 


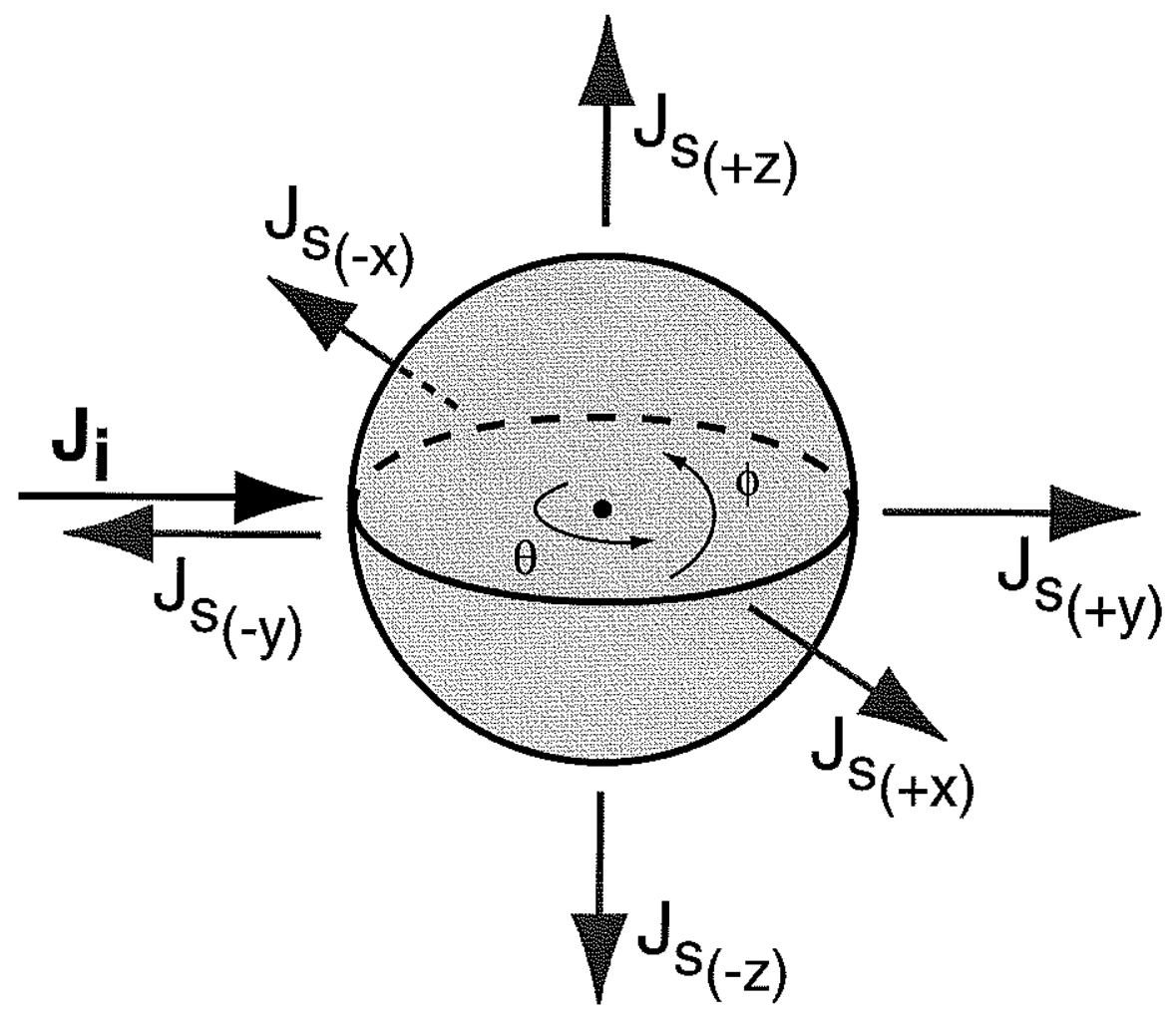

Emissivity $E=1-\left[\frac{\int^{2 \pi} \int^{2 \pi} J_{S} d \theta d \phi}{J_{i}}\right]$

Figure 4.5 


\section{Chapter 5 - Ambient Imaging at $9^{\circ}$ N East Pacific Rise and Endeavour Segment, JuAn de FuCa RIDGe}

The ALISS camera system was deployed at two hydrothermal fields in the Pacific Ocean - the Main Endeavour Field of the Juan de Fuca Ridge, and the Venture Hydrothermal Field at $9-10^{\circ} \mathrm{N}$ on the East Pacific Rise. Ambient light images were obtained of different styles of venting (flange pools, black smokers, and beehives), and of vents that varied in temperature, chemistry, and depth. The images were processed as described in Chapter 3, and were analyzed for spatial, temporal, and spectral variations.

The first deployment of the ALISS camera was at $9-10^{\circ} \mathrm{N}$ on the East Pacific Rise during a November-December 1997 Alvin dive cruise. The primary objective of this cruise was to test the ALISS camera and develop operational procedures. As a result, many of the images obtained proved to be unsuitable for detailed photometric analysis.

In June 1998, ALISS was deployed on the Endeavour Segment of the Juan de Fuca Ridge. At this time, operational procedures were established, and the Alvin pilots were well practiced in working with the ALISS camera. Therefore, this cruise focused on collecting specific data for detailed analysis of light emission at hydrothermal vents. For this reason, I will present the 1998 data from the Endeavour Segment first, and will follow with some of the best 1997 data from the East Pacific Rise.

\subsection{Fielo Sites}

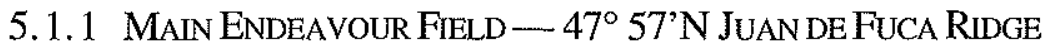

The Juan de Fuca Ridge is an intermediate rate $(6 \mathrm{~cm} / \mathrm{yr}$ full rate) spreading center in the northeastern Pacific Ocean (see Figure 1.1). It extends for $\sim 500 \mathrm{~km}$ from the Blanco Fracture Zone in the south to the Sovanco Fracture zone in the north with a strike of $020^{\circ}$. The ridge is divided into approximately six segments each $50-100 \mathrm{~km}$ in length [Johnson 
and Holmes, 1989]. The Endeavour Segment, a northern portion of the ridge, extends $\sim 90$ $\mathrm{km}$ between two overlapping spreading centers: the Cobb Offset at $\sim 47^{\circ} 40^{\prime} \mathrm{N}$ and the Endeavour Offset at $48^{\circ} 15^{\prime} \mathrm{N}$ [Delaney et al., 1992; Karsten et al., 1986]. The morphology of this segment varies from a narrow $(4 \mathrm{~km})$ axial high ( $2050 \mathrm{~m}$ depth) in the center to broad $(10 \mathrm{~km})$ deep $(2700 \mathrm{~m}$ depth) basins at both ends. The ridge axis is defined by a 1 $\mathrm{km}$ wide inner rift bounded by steep inward-facing faults [Karsten et al., 1986]. Currently, this segment appears to be in a primarily extensional mode with active faults providing pathways for hydrothermal fluids [Delaney et al., 1992].

Hydrothermal venting on the Endeavour Segment is located along the western wall of the rift valley at $47^{\circ} 57^{\prime} \mathrm{N}, 129^{\circ} 06^{\prime} \mathrm{W}$ at a depth of 2200 to $2220 \mathrm{~m}$ [Crane et al., 1985; Tivey and Delaney, 1986]. The Main Endeavour Field [described in detail by Delaney et al. , 1992] consists of a number of large (20 m x $25 \mathrm{~m} \times 20 \mathrm{~m}$ ) sulfide structures covering an area of $300 \mathrm{~m} \times 400 \mathrm{~m}$ (Figure 5.1). The large size of the sulfide structures suggests a "mature" hydrothermal system and sustained venting. In addition to high-temperature $\left(>350^{\circ} \mathrm{C}\right)$ black smokers on the tops of these structures, hydrothermal fluids are also trapped beneath sulfide-sulfate-silica flanges along the sides of the structures (see Figure 1.2B). The high-temperature (as high as $350^{\circ} \mathrm{C}$ ) fluids pooled beneath these flanges are stably stratified, forming a sharp interface with the ambient seawater below through which minimal mixing occurs [Delaney et al., 1992]. This provides a unique opportunity to image high-temperature fluids in a relatively stable, non-turbulent environment.

\subsubsection{Venture Hydrothermal Field - 950’N EAst PaciFic Rise}

The East Pacific Rise (EPR) extends southward from the Gulf of California to the Southern Ocean (see Figure 1.1) with a spreading rate that varies from intermediate (6 $\mathrm{cm} / \mathrm{yr}$ full rate) at $21^{\circ} \mathrm{N}$ [Larson, 1971$]$ to super-fast $(15 \mathrm{~cm} / \mathrm{yr}$ full rate $)$ at $20^{\circ} \mathrm{S}[$ Rea, 
1978]. The portion of the ridge between the Clipperton Transform fault $\left(10^{\circ} 20^{\prime} \mathrm{N}\right)$ and the overlapping spreading center (OSC) at $9^{\circ} 03^{\prime} \mathrm{N}$ is opening at a fast-spreading full rate of 11 $\mathrm{cm} / \mathrm{yr}$ [Klitgord and Mammerickx, 1982]. The morphology along strike varies from a 2 $\mathrm{km}$ wide, $2760 \mathrm{~m}$ deep ridge at the $9^{\circ} 03^{\prime} \mathrm{N}$ OSC to a $4 \mathrm{~km}$ wide, $2540 \mathrm{~m}$ deep ridge at $9^{\circ} 50^{\prime} \mathrm{N}$ which deepens to $2600 \mathrm{~m}$ at the Clipperton Transform [Haymon et al., 1991]. The ridge axis is identified along most of its length by an axial summit caldera (ASC) ranging from 40 to $150 \mathrm{~m}$ in width, and $<15 \mathrm{~m}$ deep - which is the result of lava drainback and gravitational collapse [Haymon et al., 1991].

The Venture Hydrothermal Field (Figure 5.2) is located within the ASC from $9^{\circ} 30^{\prime} \mathrm{N}$ to $9^{\circ} 54^{\prime} \mathrm{N}$ and consists of a number of high-temperature $\left(>350^{\circ} \mathrm{C}\right)$ black smokers as well as areas of diffuse low-temperature flow and associated biological communities [Haymon et al., 1991]. In 1991, an eruption occurred on the EPR between $9^{\circ} 45^{\prime} \mathrm{N}$ and $9^{\circ} 52^{\prime} \mathrm{N}$ which redistributed the hydrothermal venting in this area [Haymon et al., 1993]. The extremely high-temperature $\left(>400^{\circ} \mathrm{C}\right)$, low salinity fluids sampled suggest phase separation of the hydrothermal fluids beneath the surface [Von Damm et al., 1995a]. Over time, the fluids have decreased in temperature and increased in salinity [Oosting and Von Damm, 1996; Von Damm et al., 1995a]. A second, smaller eruption occurred in 1992 which affected only two of the high-temperature vents in the $9^{\circ} 50^{\prime} \mathrm{N}$ area [Oosting and Von Damm, 1996].

\subsection{AMBIENT Light IMAGING}

\subsubsection{Main Endeavour Field, Juan de Fuca Ridge}

Three black smokers and two flanges were imaged with ALISS over a six day period in June 1998: Puffer Vent, Peanut Vent, Sully Vent, Lobo Flange, and Dudley Flange (Figure 5.1, Table 5.1). The short length of the cruise prohibited repeated imaging 
of the same vents days apart (as was possible at $9^{\circ}-10^{\circ} \mathrm{N} E P R$ ); however, we were able to obtain two data sets from Puffer Vent.

Lobo and Dudley are both located in the northeastern region of the vent field where chlorinities tend to be high ( $94 \%$ of seawater cholorinity), while Puffer, Peanut, and Sully are located in the southwestern region of the vent field where the chlorinities are lower ( $47 \%$ of seawater) (see Tables 4.2 and 4.3) [Butterfield et al., 1994]. Puffer Vent and Sully Vent both appeared to be boiling. (Puffer is so named because it appears to "puff" due to phase separation). Indeed, the chemistry of the vents (e.g., the low chlorinity), in addition to the temperature and pressure conditions, indicate phase separation at or just below the seafloor [Bischoff and Pitzer, 1985; Butterfield et al., 1994]. Temperature was not obtained at Peanut Vent due to electrical problems during dive 3237.

\subsubsection{Flange Pools}

The relative stability of flange pools provide an excellent environment in which to image pure hydrothermal fluids that are not subject to turbulence, mixing with ambient seawater, and rapid precipitation of minerals. Most of the possible sources of vent light (described in Chapter 2), other than thermal radiation, are dependent on turbulence, mixing, and/or precipitation. Hence, it is predicted that light emission from flange pools is solely due to thermal radiation.

Hydrothermal fluids pooled beneath flanges can be difficult to observe. We were unable to position the ALISS camera directly beneath flanges in an upward-looking orientation. Thus, the camera frame was designed to tilt such that the camera could look up at an angle (Figure 5.3). The camera-flange distance was difficult to ascertain in this

configuration as the ranging lasers were incident on the outer portion of the flange. Also, 
due to the geometry, the portion of the flange at the top of the image is closer (and hence less affected by attenuation) than the portion of the flange at the bottom of the image.

\section{Lobo Flange}

Lobo Flange was imaged on Alvin dive 3235. The processed ALISS images of Lobo Flange taken with both filter arrays (see Figure 5.4 for the filter layout) are shown in Figure 5.5. Most of the ambient light is emitted between 700 and $900 \mathrm{~nm}$ (at wavelengths longer than $900 \mathrm{~nm}$ the attenuation in seawater becomes dominant). The dark sport in the image is material protruding through the flange into the cold, ambient seawater (as noted by observers on the dive) - thus it does not emitting significant thermal light. Also, in each tile, the upper portion of the image is brighter than the lower portion. This is due to the orientation of the camera during imaging (Figure 5.3) as noted above.

As predicted, the light from Lobo Flange appears to be purely thermal radiation. The spectrum from Lobo (Figure 5.6) shows emission from 600 to $950 \mathrm{~nm}$ that corresponds well with the predicted flux from a black body. Data in the 450,500 , and 550 $\mathrm{nm}$ bands was below the camera detection level. (Only the brightest/closest portion of the image - approximately $20 \mathrm{~cm}^{2}$ - was used in the spectral calculation). The predicted black body flux at the orifice was calculated from Equation 2.2. This flux was reduced to account for attenuation by $50 \mathrm{~cm}$ of seawater (Equation 4.1) and transmission through the ALISS optics (Figure 3.7), and then summed over each filter. The band of expected thermal radiation in Figure 5.6 includes uncertainties in distance $( \pm 10 \mathrm{~cm})$, temperature $\left( \pm 5^{\circ} \mathrm{C}\right)$, and emissivity $( \pm 0.1)$.

Emissivity was estimated from the ALISS data. If temperature is known (or assumed), the only unknown variable in Planck's Equation (Equation 2.2) is emissivity. Since, the flux at long wavelengths $(>700 \mathrm{~nm})$ is assumed to be purely thermal radiation, 
an approximate value of emissivity was determined by fitting the predicted thermal flux to the actual flux at long wavelengths. The temperature of Lobo Flange was measured to be $332^{\circ} \mathrm{C}$. Thus, an emissivity of $\sim 0.9$ is required to fit the data [White et al., 2000]. This high emissivity is to be expected as the fluid is backed by opaque rock at the same temperature as the fluid. It should be noted that emissivity may be dependent on wavelength, but this cannot be characterized with this procedure.

\section{Dudley Flange}

Dudley Flange was imaged on the following Alvin dive (3236). Unlike Lobo Flange, the image of Dudley has a large number of dark features (Figure 5.7) that appear to be sulfide material that is protruding through the flange pool into the cold ambient seawater (as noted by observers on the dive). Most of the light emission at Dudley is between 600 and $950 \mathrm{~nm}$. While the long-wavelength light corresponds to thermal radiation, excess emission was detected at $500 \mathrm{~nm}$ that is not due to thermal radiation. Spectra were determined for portions of the image $\left(\sim 11 \mathrm{~cm}^{2}\right)$ associated with and not associated with the dark features (Figure 5.8). These spectra (Figure 5.9) suggest that the emission in the 500 $\mathrm{nm}$ band is related to the dark features in the image. Since no light above the detection level was observed in the 450 and $550 \mathrm{~nm}$ bands, and the filters are $100 \mathrm{~nm}$ in bandwidth, the excess emission must be constrained to a narrow band at $500 \mathrm{~nm}$. The idea that solid material protruding through a flange pool would lead to visible light emission is not obvious. However, the existence of such material may induce local vertical mixing or precipitation on the substrate which are associated with possible emission mechanisms. 


\subsubsection{Black Smokers}

The difficulties involved with setting up the ALISS camera at a suitable vent which include vent geometry, and the need for stability - were discussed in Section 3.1.4. Despite these difficulties, we were able to obtain some extremely good quality images at black smokers. Unlike flange pools, black smokers have a much smaller emitting area. In order to relate the emission in the ambient images to the actual structure, images were obtained of the vents illuminated by Alvin's external lights. Also, an attempt was made to measure the temperature of the fluid at the orifice. Due to the sharp thermal gradients and turbulence of the vent plume, the temperature measurements fluctuated greatly. As stated in Section 4.1, careful temperature measurements made on dives 3234 and 3238 suggest that the temperatures just outside of the orifices of Puffer Vent and Sully Vent are $\sim 330^{\circ} \mathrm{C}$ and $\sim 340^{\circ} \mathrm{C}$, respectively. In order to fit the data with a theoretical curve at these temperatures (as described above), an emissivity of $\sim 0.3$ was required. Since this value provided a suitable fit to the data for both Puffer and Sully vents, it was used as the emissivity of a black smoker throughout the analyses. Indeed, the semi-transparent nature of a black smoker would suggest a relatively low emissivity as compared to a flange pool backed by hot opaque rock.

\section{Peanut Vent}

Peanut Vent was imaged on dive 3237. The ambient light image of Peanut Vent (Figure 5.10) is representative of all ALISS images of black smokers. Light is observed in the tiles corresponding to wavelengths of 700 to $950 \mathrm{~nm}$ (see Figure 5.4 for filter layout). However, it should be noted that low-intensity emission in the visible region can often not be seen in the images with the naked eye. Low-intensity data in this region is extracted through computer processing of the images. Black smokers are interesting objects to image 
because of the spatial variation of the emitted light. Figures 5.11A and 5.11B show an illuminated image of Peanut (in the $550 \mathrm{~nm}$ band) and an ambient light image (in the 870 $\mathrm{nm}$ band), respectively. The highest intensity light is associated with the orifice and intensity decreases with distance away from the orifice.

The strongest signal is observed in the $870 \mathrm{~nm}$ band, and the light in this band is assumed to be primarily thermal radiation. Thus, the variation in light intensity in this band can be used to indicate variation in temperature. In analyzing the spectrum of black smoker light, we isolate portions of the plume by intensity (or temperature). As mentioned in Section 3.2 , the $870 \mathrm{~nm}$ image is used to extract a source mask which isolates the emitting region from the background. This source mask is then applied to all wavelength tiles. Figure 5.11C shows how the pixels in the source area can be separated by intensity level. The $25 \%$ highest intensity pixels (labeled at $75-100$ percentile in the figure) are clustered around the orifice, while the pixels with the lowest intensity (labeled $0-25$ percentile) are found along the fringes of the source mask. We can then look at the spectra from each of these regions (Figure 5.12A). The highest intensity light corresponds well to a $340^{\circ} \mathrm{C}$ black body with an emissivity of 0.3 at long wavelengths. The long-wavelength light from the lower intensity regions decreases as expected for decreasing temperatures. The spectrum from the $0-25$ percentile region corresponds well to a $270^{\circ} \mathrm{C}$ black body. The predicted black body emission bands include uncertainties in temperature $\left( \pm 10^{\circ} \mathrm{C}\right)$, distance $( \pm 5 \mathrm{~cm})$, and emissivity $( \pm 0.1)$. No significant light is observed at short wavelengths $(<600 \mathrm{~nm})$.

A second set of images was obtained at Peanut Vent while an iron bar was held in the plume to disrupt the flow. The presence of the bar affected the spectral emission at Peanut Vent in two ways (Figure 5.12B). First, at wavelengths above $650 \mathrm{~nm}$, the emission decreased by about $19 \%$. Presumably this is due to increased entrainment of 
ambient seawater which slightly lowered the temperature. Second, at wavelengths below $650 \mathrm{~nm}$, emission increased slightly. In the hottest/brightest region, the emission increased by $27 \%$ at $600 \mathrm{~nm}$. Thus, the increased mixing may have activated a non-thermal mechanism of light emission, or increased the emission of such a mechanism. However, the light observed at shorter wavelengths is not significantly above that expected for thermal radiation.

\section{Puffer Vent}

Puffer Vent was imaged on two Alvin dives three days apart (dives 3234 and 3237). Puffer appeared to be boiling, and a maximum temperature of $372^{\circ} \mathrm{C}$ was measured inside the orifice. Unlike most black smokers where the fluid exits from the top of a chimney-like structure, the fluid at Puffer Vent is directed downward from a large sulfide structure and then rises buoyantly (Figure 5.13). It is difficult to see the orifice under illuminated conditions (Figure 5.14A). However, ambient light images show quite clearly where the highest temperature (and hence the orifice) is located (Figure 5.14B). As with Peanut Vent, the ambient light images of Puffer Vent in the $870 \mathrm{~nm}$ band - from dives 3234 (Figure 5.14B) and 3237 (Figure 5.15A) - were divided into regions based on intensities (Figures 5.14C and 5.15B ). The spectra from these regions are plotted in Figure 5.16 .

The spectra from Puffer Vent show correlation with thermal radiation at long wavelengths $(\geq 600 \mathrm{~nm})$. The light emission at these wavelengths corresponds to temperatures of $\sim 330-340^{\circ}$ down to $270^{\circ} \mathrm{C}$. However, distinct differences are seen in the data from the two dives. The data from dive 3234 (Figure 5.16A) show significant light emission $\left(\sim 10\right.$ photons $\left./ \mathrm{sec} / \mathrm{cm}^{2}\right)$ from $500-550 \mathrm{~nm}$ in the $50-75$ percentile region $(2-2.5$ $\mathrm{cm}$ up the plume) [White et al., 2000]. Approximately 2 photons $/ \mathrm{sec} / \mathrm{cm}^{2}$ are observed 
from $400-450 \mathrm{~nm}$ and $550-600 \mathrm{~nm}$ in the $0-25$ and $25-50$ percentile regions. The flux in the $900 \mathrm{~nm}$ band is also higher than expected for a thermal source. On dive 3237, no significant flux is observed below $600 \mathrm{~nm}$ (Figure 5.16B). Additionally, a higher flux is observed at long wavelengths on dive 3237 than on dive 3234 (corresponding to a temperature of $340^{\circ}$ rather than $330^{\circ} \mathrm{C}$ ). From 650 to $870 \mathrm{~nm}$, the flux observed at the orifice (the 75-100 percentile region) more than doubles from dive 3234 to 3237 . It is not clear why the short-wavelength light decreases while the long-wavelength light increases from one dive to the next.

McDuff and Delaney [1995] have shown that Puffer Vent experiences variations in temperature and chemistry that correlate with the semidiurnal tides $(\sim 12.42$ hours $)$ decreasing temperature is accompanied by slight increases in $\mathrm{Cl}$ and $\mathrm{Li}$ concentration. (Tidal variability is also seen in diffuse flow at Endeavour, TAG, and Guaymas Basin [Schultz and Elderfeld, 1997, and sources therein]). The two ALISS data sets from Puffer Vent were collected $\sim 68$ hours apart (offset $\sim 5$ hours in the tidal cycle). On dive 3234 , a temperature of $372^{\circ} \mathrm{C}$ was recorded inside the orifice. No temperature measurements were made on dive 3237 due to electrical failure. However, the ambient light data suggest that the temperature was $\sim 10^{\circ} \mathrm{C}$ higher than on dive 3234. Although Puffer Vent is known to experience tidal variability, the spacing of the ALISS data sets (3 three days apart) cannot distinguish short-term from long-term variations in light emission.

\section{Sully Vent}

Sully Vent was imaged on dive 3238. Sully, like Puffer, appeared to be boiling and an internal orifice temperature of $373^{\circ} \mathrm{C}$ was recorded (Table 5.1). The temperature just outside of the orifice was $\sim 340^{\circ} \mathrm{C}$. In addition to the standard 5-minute exposure images, a series of sixty $30-$ second exposure images were obtained over a 30 minute 
period to look at temporal variations on short time scales. The combination of images obtained at Sully Vent reveal some interesting variations in light emission at vents.

The black smoker imaged at Sully vent actually consisted of two orifices close together (Figures 5.17A and B). The source area was divided into regions based on intensity (Figure 5.17C) and the spectra from those regions are shown in Figure 5.18. The long-wavelength light $(\geq 600 \mathrm{~nm})$ corresponds to thermal radiation from a body of $\sim 340^{\circ} \mathrm{C}$ down to $\sim 270^{\circ} \mathrm{C}$. Unlike both Puffer and Peanut Vents, significant light is observed at the orifice (in the 75-100 percentile region) between 400 and $550 \mathrm{~nm}$ - approximately 6-8 photons $/ \mathrm{sec} / \mathrm{cm}^{2}$. (The 500 and $900 \mathrm{~nm}$ filters were not used). The flux remains significant at those wavelengths in the lower intensity regions.

Approximately two hours before the 5-minute exposures (discussed above) were obtained, a number of 30-second exposure images were obtained over a 30 minute period. The spectra from those exposures are shown in Figure 5.19A. (Note that spectra are for the entire source aperture - not divided by intensity.) A time series of the data (Figure 5.19B) shows slight periodic variation in each band (most likely caused by pulsations in the plume due to boiling). The most striking feature is an increase in flux (by $\sim 50$ photons $\left./ \mathrm{sec} / \mathrm{cm}^{2}\right)$ at $\sim 15$ minutes seen in the shortest wavelength bands $(450,550$, and 599 $\mathrm{nm}$ ) [White et al., 2000]. No significant shift is seen at the long wavelengths. The fact that long wavelengths are not affected rules out a change in camera-vent distance as the cause. By the time the 5-minute exposures were obtained ( 2 hours later), the flux at the shorter wavelengths had dropped back to values comparable to those of the first shortexposure images (Figure 5.18). Thus, temporal changes observed at Puffer and Sully over time periods of days and hours can occur on time scales of minutes. 


\subsubsection{Venture Hydrothermal Field, 9-10 ${ }^{\circ} \mathrm{N}$ EAst PaciFic Rise}

ALISS was used to image five vents in the Venture Hydrothermal Field - P Vent, Q Vent, V Vent, and L Vent (AdV 4-9) — during a November-December 1997 Alvin cruise. The primary purpose of this cruise was to test and develop procedures for the ALISS camera system. Thus, only some of the data were properly obtained for performing detailed photometric analyses. I will present one set of data from each of the vents - all of which are high-temperature black smokers - and data from a "beehive" structure at Q Vent.

\subsubsection{Black Smokers}

As with the black smokers from the Endeavour segment, an emissivity of 0.3 is used for all of the analyses since emissivity cannot be well constrained and the temperature just outside of the orifice is difficult to measure. By assuming a constant value of emissivity, the temperature required to fit the data with a theoretical black body can vary from vent to vent (as was seen in the section above). This may represent actual variations in temperature, or may be a consequence of variations in emissivity from vent to vent. At this point we cannot distinguish between the two.

\section{$P$ Vent}

P Vent was imaged on Alvin dives $3171,3173,3178$, and 3182 . Only the images from dive 3182 are shown here (Figure 5.20), as the earlier images suffer from blurriness, not being completely within ALISS's field of view, and short exposure times. The temperature at $\mathrm{P}$ Vent was rather consistently measured to be $377^{\circ} \mathrm{C}$ (Table 5.2). During the early dives to $\mathrm{P}$ Vent parts of the chimney were knocked off to allow the temperature probe to be inserted easily into the orifice. On returning to $\mathrm{P}$ Vent on dive 3182 , we 
discovered that an intricate "candelabra" structure had built up from the orifice (sulfide precipitation at vents can be extremely rapid [Hekinian et al., 1983]). While the plume hides most of this feature from view (Figure 5.21A), it is clearly seen in the ambient light image (Figure 5.21B). The source area was divided into regions based on intensity (Figure 5.21C), and the spectra from each region are shown in Figure 5.22.

The long-wavelength light $(\geq 600 \mathrm{~nm})$ at the orifice of $\mathrm{P}$ Vent $(75-100$ percentile region) is consistent with thermal radiation from a $345 \pm 10^{\circ} \mathrm{C}$ black body of emissivity $0.3 \pm 0.1$ at a distance of $50 \pm 5 \mathrm{~cm}$. The lower intensity regions correspond to thermal radiation from lower temperature black bodies, down to a minimum of $265^{\circ} \mathrm{C}$. Light that exceeds the expected thermal flux is observed at $500-550 \mathrm{~nm}\left(\sim 2-4\right.$ photons $\left./ \mathrm{sec} / \mathrm{cm}^{2}\right)$ for the $0-25,25-50$, and $50-75$ percentile regions.

$V$ Vent

$\mathrm{V}$ Vent was imaged on dive 3186 (Figure 5.23). Venting was observed both on a large sulfide structure and atop a 3-4 m high sulfide pillar. The upper vent was $339^{\circ} \mathrm{C}$, but we were unable to maintain a stationary position for ALISS imaging. Thus, we imaged the lower vent $\left(315^{\circ} \mathrm{C}\right)$. Three small orifices are visible in the ALISS image (Figure 5.24A and B). Although the center vent was the prime target, we imaged enough of the right-hand vent to analyze it as well. The breakdown of the regions analyzed is shown in Figure $5.24 \mathrm{C}$. The source area was much smaller than the previously imaged vents and, thus, only two regions (0-50 and 50-100 percentile) were analyzed ( $3 \mathrm{~cm}^{2}$ each).

The spectra of both orifices are shown in Figure 5.25. The center vent corresponds to a $265 \pm 10^{\circ} \mathrm{C}$ black body at long-wavelengths ( $\geq 650 \mathrm{~nm}$ ). From $500-550 \mathrm{~nm}$ a significant amount of light, two orders of magnitude greater than expected for purely thermal radiation, is observed in the 50-100 percentile region $\left(\sim 3-5\right.$ photons $\left./ \mathrm{sec} / \mathrm{cm}^{2}\right)$. The 
right-hand vent is much different from the center vent. Assuming the same emissivity $(0.3 \pm 0.1)$ and distance $(50 \pm 5 \mathrm{~cm})$, a higher temperature black body $\left(\sim 275^{\circ} \mathrm{C}\right)$ is required to fit the data. In fact, this orifice is further away from the ALISS camera, and thus its temperature must be even higher $\left(\sim 295^{\circ} \mathrm{C}\right.$ at $\left.60 \mathrm{~cm}\right)$. Also, while some light is observed in the 500 and $550 \mathrm{~nm}$ bands, it is not significantly above the detection level. However, a significant amount of light is observed in the $450 \mathrm{~nm}$ band $\left(\sim 3\right.$ photons $\left./ \mathrm{sec} / \mathrm{cm}^{2}\right)$ and is the same for both regions (0-50 and 50-100 percentile).

The differences seen in the light emission from two adjacent vents is somewhat puzzling. One would expect the fluid exiting from both orifices to have traveled much the same path and therefore experienced similar amounts of subsurface mixing and have similar chemistries. Only water from the upper, higher-temperature orifice was collected from sampling, thus the chemistry of the two vents imaged is not clearly known. While temperature measurements were not made at the right-hand vent, the long-wavelength light suggests that it was $10-30^{\circ} \mathrm{C}$ hotter than the center vent at the time of ALISS imaging.

\section{$L$ Vent (AdV 4-9)}

The L Vent area actually consists of a number of vents, all of which were individually marked during the AdVenture dive series. The black smoker AdV 4-9 (hereafter referred to as L Vent) was imaged by ALISS on dive 3191 (Figure 5.26). The internal orifice temperature was measured to be $315^{\circ} \mathrm{C}$ with the Alvin hi-T probe (Table 5.2). The illuminated image (Figure 5.27A) shows black smoke exiting from a 5-cm wide chimney. The ambient light image (Figure 5.27B) reveals two actual orifices in the chimney. The source area of the light emission is a cone extending from the orifice to the top of the ALISS image $(\sim 10 \mathrm{~cm})$. This area is divided into regions based on intensity (Figure 5.27C) and the spectra from each region are plotted in Figure 5.28. 
At long wavelengths ( $\geq 650 \mathrm{~nm}$ ) the light emission corresponds well to a black body of emissivity $0.3 \pm 0.1$ with a temperature ranging from $295^{\circ} \mathrm{C}$ to a low of $260^{\circ} \mathrm{C}$. (Measurements made on dive 3073 during an April 1996 cruise confirm a temperature of $295^{\circ} \mathrm{C}$ at the orifice corresponding to an internal orifice temperature of $\sim 311^{\circ} \mathrm{C}$ ). At shorter wavelengths ( $\leq 600 \mathrm{~nm}$ ), a flux of $\sim 2-4$ photons $/ \mathrm{sec} / \mathrm{cm}^{2}$ is seen across all bands in the hottest/brightest regions (50-75 and 75-100 percentile). This type of white light was previously observed only at Sully Vent. In the $25-50$ percentile region, flux decreases from $\sim 2$ photons $/ \mathrm{sec} / \mathrm{cm}^{2}$ at $450 \mathrm{~nm}$ to $\sim 0.35$ photons $/ \mathrm{sec} / \mathrm{cm}^{2}$ at $600 \mathrm{~nm}$. This trend looks similar to emission from Cerenkov radiation (Figure 2.4). However, the amount of Cerenkov radiation expected (for either ${ }^{40} \mathrm{~K}$ or ${ }^{222} \mathrm{Rn}$ ) is orders of magnitude less than what is observed. In the $0-25$ percentile region, no light is observed below $650 \mathrm{~nm}$.

\section{QVent}

A $342^{\circ} \mathrm{C}$ black smoker at Q Vent was imaged on dive 3187 (Table 5.2). The camera was centered on the actual chimney (Figure 5.29A), and thus the light emission was not entirely within ALISS's field of view (Figure 5.29B). Figure 5.29C shows the division of the source area into four regions. Since the top portion of the plume was not imaged, the spectra from the regions will show how light varies across a plume rather than up a plume. The spectra are shown in Figure 5.30. The light emission from Q Vent corresponds to thermal radiation from a $270-320^{\circ} \mathrm{C}$ black body with an emissivity of $0.3 \pm 0.1$ at all wavelengths. No light is observed above the detection level at short wavelengths $(<650 \mathrm{~nm})$. 


\subsubsection{2 "Beehive" Structure}

A beehive structure was also present at Q Vent, and was imaged on dive 3174 (no temperature measurements were made). Beehives have been observed at hydrothermal fields on the Mid-Atlantic and Juan de Fuca Ridges [Tivey, 1995, and references therein]. Unlike chimneys, beehives have a bulbous morphology and high porosity ( $30 \%$ [Koski et $a l ., 1994])$. The structure itself is primarily a matrix of anhydrite, with lesser amounts of pyrite, chalcopyrite, sphalerite, and wurtzite. Clear hydrothermal fluid percolates though the beehive and emanates from the sides of the structure (Figure 1.2C). No significant mixing or black smoker fluid is observed [Tivey, 1995].

The beehive at Q Vent and the light emitted from it are shown in Figures 5.31A and 5.31B. The beehive is on the left third of the images. Dividing the source area by intensity does not isolate significant portions of the beehive (and the spectra from those regions do not vary significantly). Thus, the entire structure was analyzed as one unit (identified as "beehive structure" in Figure 5.31C). In an effort to analyze pure fluid without rock behind it, the area outside of the source region but within one or two centimeters of it was isolated (identified as "fluid layer" in Figure 5.31C).

The spectra from the beehive and the fluid layer are shown in Figure 5.32. Light flux from the beehive corresponds well to a $265^{\circ} \mathrm{C}$ black body of emissivity $0.9 \pm 0.1$ at wavelengths $\geq 700 \mathrm{~nm}$. An emissivity of 0.9 was used because the fluid imaged is backed by hot opaque rock, similar to a flange pool. Additionally, $\sim 3$ photons $/ \mathrm{sec} / \mathrm{cm}^{2}$ are observed in the 500 and $550 \mathrm{~nm}$ bands, and $\sim 8$ photons $/ \mathrm{sec} / \mathrm{cm}^{2}$ is observed in the $600 \mathrm{~nm}$ band. Assuming the temperature of the fluid layer is the same as the beehive $\left(265^{\circ} \mathrm{C}\right)$, an emissivity of 0.1 is required to fit the data. (This low value of emissivity is not surprising given the low absorptivity of clear water). However, flux from the fluid layer deviates from that of a black body in two ways. First, the flux at $800 \mathrm{~nm}$ is too low by a factor of 
3. A similar dip at $800 \mathrm{~nm}$ is present in the beehive spectra as well. This type of decrease in long-wavelength light was not observed at other vents and cannot be easily explained. Secondly, in the $600 \mathrm{~nm}$ band a similar amount of light is observed in the fluid layer $(\sim 7$ photons $/ \mathrm{sec} / \mathrm{cm}^{2}$ ) as is observed from the beehive. Comparing light emission from the beehive structure to that from the fluid itself suggests that the excess light at $600 \mathrm{~nm}$ is due to a fluid-related mechanism, such as mixing or turbulence, while the excess emission at 500 and $550 \mathrm{~nm}$ observed only at the beehive may be related to mineral-related mechanism, such as precipitation.

\subsection{Discussion}

A summary of the ambient light data obtain with the ALISS instrument is given in Table 5.3. The ALISS data show that light is ubiquitous at all types of high-temperature hydrothermal vents (i.e., flange pools, black smokers, and beehives), and that thermal radiation is the dominant source. This radiation is dependent entirely on the temperature of the fluid being imaged and its emissivity. Since flange pools and beehive structures are backed by hot, opaque rock, they are expected to have a higher emissivity than the semitransparent fluid of a black smoker. Emissivity was estimated from the ALISS data by assuming that long-wavelength light emission was purely thermal radiation. Flange pools have an emissivity of $\sim 0.9$. The emissivity of black smoker fluid appears to be much less ( $\sim 0.3$ ), and clear hydrothermal fluid (such as that emanating from a beehive) may be as low as 0.1 . It should be noted that emissivity may be dependent on wavelength, but this cannot be characterized from the ALISS data.

Light emission from a flange pool appears to be primarily thermal radiation. The high emissivity suggests that a significant amount of radiation is being emitted by the hot rock behind the fluid. Lobo Flange and Dudley Flange both emit light consistent with 
thermal radiation from a $332^{\circ} \mathrm{C}$ body of emissivity 0.9 (Table 5.3). Some flanges have solid material protruding through the hot flange pool into cold ambient seawater. This is seen as dark features in the ambient light images (e.g., Figure 5.7). At Dudley Flange, an excess light emission in the $500 \mathrm{~nm}$ band $\left(\sim 2\right.$ photons $\left./ \mathrm{cm}^{2} / \mathrm{sec}\right)$ is observed in the vicinity of this material. It is possible that precipitation is actively occurring on these features (causing crystalloluminescence), or that they induce local mixing (causing chemiluminescence). The confinement of the emission to a narrow band at $500 \mathrm{~nm}$, suggests that only one mechanism is generating the light. Analyses of the protruding material may help to determine whether processes such as crystalloluminescence or chemiluminescence are occurring. Thus, in areas where hydrothermal fluid is not subject to turbulence, mixing, or precipitation the only emitted radiation appears to be due to the temperature of the fluid and rock.

The dominant source of light emission at black smokers is also thermal radiation. While the maximum temperature inside a black smoker chimney is relatively easy to measure, that of the fluid outside of the orifice is much more difficult to determine. The ALISS data suggest that, in most cases, the fluid temperature drops by $20-30^{\circ} \mathrm{C}$ on exiting the orifice. ALISS provides a way of observing spatial temperature variations in a plume by looking at the distribution of thermal radiation. In many cases, while the plume of a smoker widens as it rises, the emitted light (and hence higher temperature fluid) is confined to a narrowing cone above the orifice. The ALISS data show three cases of purely thermal radiation at black smokers (Table 5.3): Peanut Vent (dive 3237), Puffer Vent (dive 3237), and Q Vent (dive 3174).

Some black smoker vents also emit non-thermal light. Repeated measurements at Puffer Vent and Sully Vent suggest that this light can vary greatly over time [White et al., 2000]. This light is significant at short-wavelengths $(\leq 650 \mathrm{~nm})$ and may be caused by a 
number of mechanisms related to turbulence, mixing, and/or precipitation (as discussed in Chapter 2). The spatial and spectral data obtained with the ALISS camera can be used to infer which sources are responsible for the non-thermal light. Most non-thermal light is associated with the cooler regions of the plume. This can be seen at Puffer Vent, Sully Vent, $\mathrm{P}$ Vent, $\mathrm{V}$ Vent, and L Vent where significant light is observed away from the orifice $(0-25,25-50$, and 50-75 percentile regions) at $\sim 500-600 \mathrm{~nm}$. This suggests a mechanism related to mixing (chemiluminescence) or precipitation (crystallo- or triboluminescence). As hydrothermal fluid exits a vent, it entrains and mixes with oxygenated, ambient $\left(\sim 2^{\circ} \mathrm{C}\right)$ seawater. This mixing and associated cooling lead to sulfide oxidation and the precipitation of minerals such as sphalerite [e.g., Baron, 1998]. Sulfide oxidation is known to produce chemiluminescence in the visible region [Tapley et al., 1999]; however, its spectrum is not yet characterized. Sphalerite is known to be TL-active and luminesces at $~ 550-600 \mathrm{~nm}$ [Nelson, 1926]. Thus, both chemiluminescence (CL) and crystallo- and tribo luminescence (XTL and TL) are very likely sources of non-thermal light at black smokers (Table 5.3).

In some cases excess light over thermal radiation is observed at the orifice (75-100 percentile region). At Sully Vent and L Vent, significant light is observed from 450-600 $\mathrm{nm}$ at the orifice (75-100 percentile region). These vents do not appear to be different from the other vents in any way (i.e., in chemistry, temperature, size) so their light emission is quite perplexing. As no proposed source mechanism is known to emit white light (i.e., uniform light across all wavelengths), that observed at Sully and L Vent is most likely due to a combination of a number of sources all emitting a similar intensity in different wavelength bands. A photon flux of $\sim 2-8$ photon $/ \mathrm{cm} 2 / \mathrm{sec}$ is observed in the $400-450 \mathrm{~nm}$ range at the right-hand V Vent, as well as at Sully Vent and L Vent, and it is directly associated with orifice (75-100 percentile region). In Section 2.4 , it was noted that 
photoluminescence experiments on chimney pieces revealed strong emissions from the inside of chimneys (predominantly chalcopyrite) at $450 \mathrm{~nm}$. Thus, it is possible that at some vents we are observing the signal from mineral precipitation within the chimney conduit.

Only one beehive structure was imaged with the ALISS camera. An attempt was made to analyze light emission from both the structure itself, and the fluid emanating from it. A flux of $\sim 7-8$ photons $/ \mathrm{cm}^{2} / \mathrm{sec}$ was observed from both the structure and the fluid at $600 \mathrm{~nm}$ (Table 5.3). This appears to be a emission generated in the fluid layer (and thus superimposed on the structure emission). Because the fluid appears to be clear (i.e., no significant precipitation is observed), chemiluminesence (possibly due to sulfide oxidation) is the most likely source mechanism. The ALISS data from the structure shows significant light emission in the 500 and $550 \mathrm{~nm}$ bands $\left(\sim 3\right.$ photons $\left./ \mathrm{cm}^{2} / \mathrm{sec}\right)$ above that predicted for thermal radiation (Table 5.3). This emission could also be due to chemiluminescence, or to crystalloluminescence due to precipitation of minerals on the beehive structure.

Due to the complexity of hydrothermal systems and the lack of detailed information on possible mechanisms, we cannot confirm the extent to which any non-thermal source contributes to vent light. Non-thermal light appears to be significant in the visible region of the spectrum. Indeed, many of the mechanisms mentioned in Chapter 2 (e.g., triboluminescence, and chemiluminescence) are known to emit light in the visible region, and are associated with minerals and chemicals known to exist at hydrothermal vents. However, until these sources and vent properties can be better characterized, possible source mechanisms cannot be further constrained. 
Table 5.1 - Endeavour Vents Imaged by ALISS

\begin{tabular}{|l|c|c|c|c|c|}
\hline \multicolumn{1}{|c|}{ Vent } & Alvin & Depth & Temperature & \multicolumn{2}{c|}{ No. of Images } \\
& Dive & $(\mathbf{m})$ & $\left({ }^{\circ} \mathbf{C}\right)$ & filter 1 & filter 2 \\
\hline \hline Puffer vent & $3234 / 3237$ & 2194 & $372^{\circ} /-^{\mathrm{a}}$ & $5 / 5$ & $5 / 5$ \\
\hline Peanut vent & 3237 & 2193 & $-^{\mathrm{a}}$ & 3 & 0 \\
\hline Sully vent & 3238 & 2189 & $373^{\circ}$ & $7 / 60^{\mathrm{b}}$ & 0 \\
\hline Lobo flange & 3235 & 2192 & $332^{\circ}$ & 5 & 5 \\
\hline Dudley flange & 3236 & 2186 & $332^{\circ}$ & 5 & 4 \\
\hline
\end{tabular}

${ }^{a}$ Due to electrical failure, no temperatures were obtained on dive 3237 .

${ }^{\mathrm{b}}$ Sixty 30 -sec. exposures were obtained in addition to the seven 5 -min. exposures.

Table 5.2 - East Pacific Rise Vents Imaged by ALISS

\begin{tabular}{|l|c|c|c|c|c|}
\hline \multicolumn{1}{|c|}{ Vent } & Alvin & Depth & Temperature & \multicolumn{2}{c|}{ No. of Images } \\
& Dive & $(\mathbf{m})$ & $\left({ }^{\circ} \mathbf{C}\right)$ & filter 1 & filter 2 \\
\hline \hline P vent & 3182 & 2510 & $377^{\circ}$ & 2 & 2 \\
\hline Q vent & 3187 & 2504 & $341^{\circ}$ & 3 & 3 \\
\hline \multicolumn{1}{|c}{ Beehive } & 3174 & 2504 & - & 2 & 2 \\
\hline L vent (4-9) & 3191 & 2527 & $315^{\circ}$ & 3 & 3 \\
\hline V vent & 3186 & 2514 & $315^{\circ}$ & 3 & 2 \\
\hline
\end{tabular}


Table 5.3 - Ambient Light Data

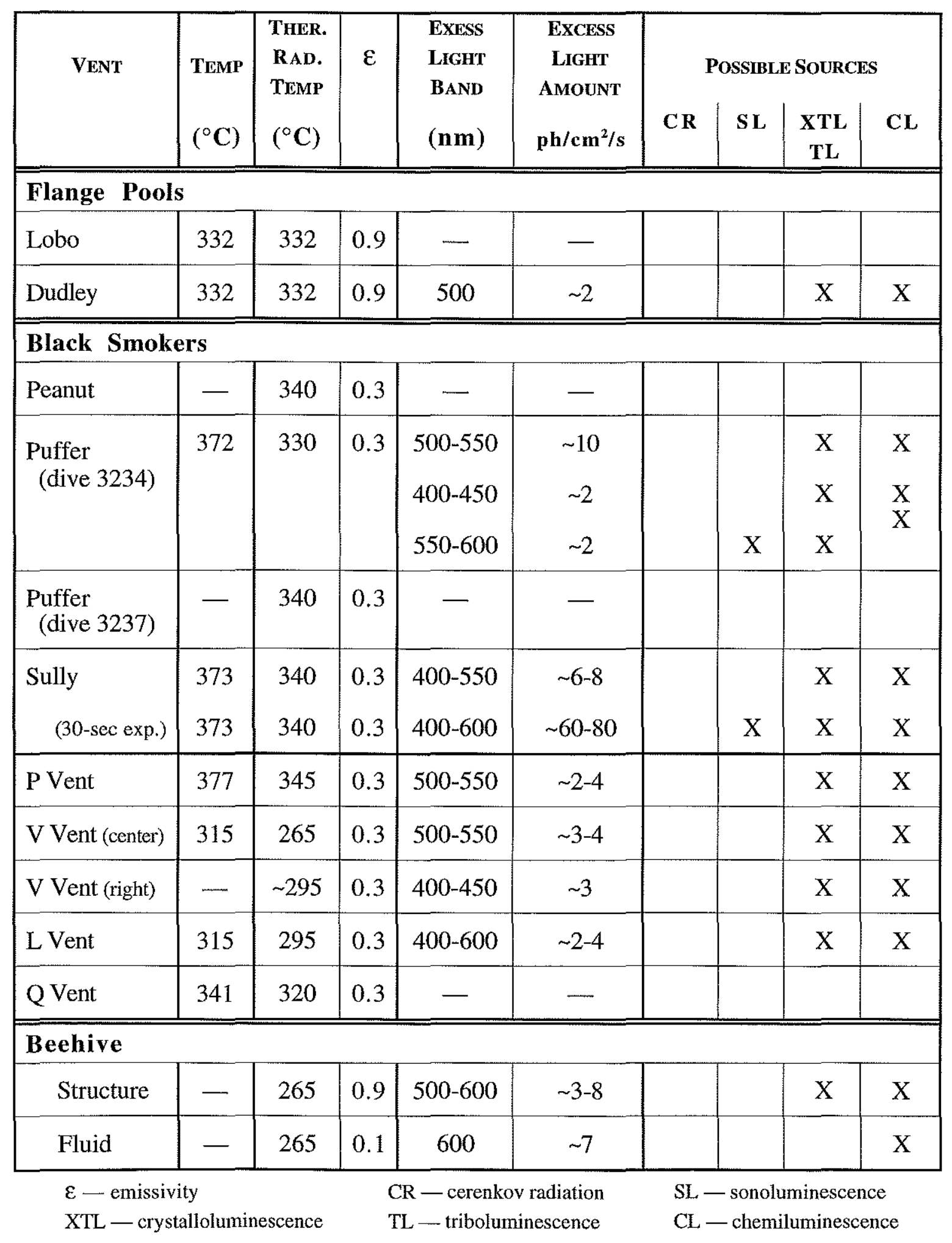




\section{Figure Captions}

Figure 5.1 Map of the Main Endeavour Field (MEF), Juan de Fuca Ridge [Modified from Delaney et al., 1992]. The MEF is located along the western wall of the axial valley. Lobo and Dudley are located in the northeastern region of the vent field, and Peanut, Sully, and Puffer are located in the southwestern region of the vent field.

Figure 5.2 Map of the Venture Hydrothermal Field at $9-10^{\circ} \mathrm{N}$ on the East Pacific Rise [Modified from Gregg et al., 1996]. A) The Venture Hydrothermal Field is located on the EPR between the Clipperton (C), and Siqueiros (S) transforms. B) High temperature vents are indicated by black dots. Labeled vents were imaged by ALISS.

Figure 5.3 Cartoon showing ALISS positioning at flange pools (A) and black smokers (B). The ranging lasers cross at a distance of $50 \mathrm{~cm}$ to aid the pilot in positioning the camera the proper distance from the vent.

Figure 5.4 The layout of the two ALISS filter arrays. The bold number indicates the center wavelength, and the number in parentheses indicates the bandwidth (in nanometers).

Figure 5.5 Processed ALISS images of Lobo Flange taken with both filter arrays. The layout of the filters is given in Figure 5.4. The top of each individual tile is brighter because it is closer to the camera and thus less attenuated. The dark feature is material that is protruding through the flange pool into the colder ambient seawater. 
Figure 5.6 Spectral data from Lobo flange. Data from the $100 \mathrm{~nm}$ bandwidth filters are compared to the calculated flux for a theoretical black body of temperature $332 \pm 5 \mathrm{C}$ and emissivity $0.9 \pm 0.1$ at a distance of $50 \pm 10 \mathrm{~cm}$ as observed through the ALISS optics. The drop-off in flux above $870 \mathrm{~nm}$ is due to the high attenuation of light in seawater at those wavelengths (see Figure 4.3). The higher flux predicted at $450 \mathrm{~nm}$ for a theoretical black body is due to a red (long-wavelength) leak in that filter. The ALISS data from the 450, 500 , and $550 \mathrm{~nm}$ filters are below the detection level.

Figure 5.7 Processed ALISS images of Dudley Flange taken with both filter arrays. The layout of the filters is given in Figure 5.4. The top of each individual tile is brighter because it is closer to the camera and thus less attenuated. The dark features are material that is protruding through the flange pool into the colder ambient seawater.

Figure 5.8 Image of Dudley flange from the $870 \mathrm{~nm}$ filter. The boxes indicate areas that were isolated for spectral analysis - A) away from dark features, B) including dark features.

Figure 5.9 Spectral data from Dudley Flange. Data are from the $100 \mathrm{~nm}$ bandwidth filters from an area not associated with the dark features (A), and an area associated with the dark features (B). The band shows calculated flux for a theoretical black body of temperature $332 \pm 5^{\circ} \mathrm{C}$ and emissivity $0.9 \pm 0.1$ at a distance of $50 \pm 10 \mathrm{~cm}$ from the ALISS camera. The drop-off in flux above $870 \mathrm{~nm}$ is due to the high attenuation of light in seawater at long wavelengths. The higher flux expected at $450 \mathrm{~nm}$ for a theoretical black body is due to a red leak in that filter. 
Figure 5.10 Processed ALISS image of Peanut Vent taken with filter array \#1. The layout of the filter array is given in Figure 5.4.

Figure 5.11 Spatial distribution of light at Peanut vent (dive 3237). A) Image of Peanut vent taken under artificial illumination in the $550 \mathrm{~nm}$ band. (The image is slightly out of focus). B) Image of ambient light emission from Peanut vent in the $870 \mathrm{~nm}$ band. C) Portions of the source mask isolated for spectral analysis. Note that 300 pixels corresponds to $\sim 15 \mathrm{~cm}$.

Figure 5.12 Spectral data from Peanut vent (dive 3237). Data for an undisturbed vent (A) and with an iron bar in the plume (B) from filter array \#1 are plotted for each of the regions shown in Figure 5.11C. The data are compared to the calculated flux for a theoretical black body of temperature $340 \pm 10^{\circ} \mathrm{C}$ and emissivity $0.3 \pm 0.1$ at a distance of $50 \pm 5 \mathrm{~cm}$ from the ALISS camera. The drop-off in flux above $870 \mathrm{~nm}$ is due to the high attenuation of light in seawater at those wavelengths. The $75-100$ percentile pixels correspond to a $340^{\circ} \mathrm{C}$ black body while the $0-25$ percentile pixels correspond to a $270^{\circ} \mathrm{C}$ black body.

Figure 5.13 Photograph of ALISS at Puffer Vent, taken through the pilot's viewport (dive 3237).

Figure 5.14 Spatial distribution of light at Puffer vent (dive 3234). A) Image of Puffer Vent taken under artificial illumination in the $550 \mathrm{~nm}$ band. B) Image of ambient light emission from Puffer Vent in the $870 \mathrm{~nm}$ band. C) Portions of the source mask isolated for spectral analysis. Note that 300 pixels corresponds to $\sim 15 \mathrm{~cm}$. 
Figure 5.15 Spatial distribution of light at Puffer Vent (dive 3237). A) Image of ambient light emission from Puffer Vent in the $870 \mathrm{~nm}$ band. B) Portions of the source mask isolated for spectral analysis. Note that 300 pixels corresponds to $\sim 15 \mathrm{~cm}$.

Figure 5.16 Spectral data from Puffer vent. Data from the $100 \mathrm{~nm}$ bandwidth filters are plotted for each of the regions shown in Figures 5.14C and 5.15B. The 3234 data (A) are compared to the calculated flux for a theoretical black body of temperature $330 \pm 10^{\circ} \mathrm{C}$ and emissivity $0.3 \pm 0.1$ at a distance of $50 \pm 5 \mathrm{~cm}$ from the ALISS camera. The dive 3237 data (B) are compared to a $340^{\circ} \mathrm{C}$ black body with the same uncertainties. The drop-off in flux above $870 \mathrm{~nm}$ is due to the high attenuation of light in seawater at those wavelengths. The $0-25$ percentile pixels correspond to a temperature of $270^{\circ} \mathrm{C}$.

Figure 5.17 Spatial distribution of light at Sully Vent (dive 3237). A) Image of Sully Vent taken under artificial illumination in the $550 \mathrm{~nm}$ band. B) Image of ambient light emission from Sully Vent in the $870 \mathrm{~nm}$ band. (Note that the camera shifted slightly between images (A) and (B). C) Portions of the source mask of the right-hand orifice isolated for spectral analysis. Note that 300 pixels corresponds to $\sim 15 \mathrm{~cm}$. 
Figure 5.18 Spectral data from Sully Vent (dive 3238). Data from filter array \#1 are plotted for each of the regions shown in Figure 5.17C. The data are compared to the calculated flux for a theoretical black body of temperature $340 \pm 10^{\circ} \mathrm{C}$ and emissivity $0.3 \pm 0.1$ at a distance of $50 \pm 5 \mathrm{~cm}$ from the ALISS camera. The drop-off in flux above $870 \mathrm{~nm}$ is due to the high attenuation of light in seawater at those wavelengths. The $0-25$ percentile pixels correspond to a temperature of $270^{\circ} \mathrm{C}$.

Figure 5.19 Temporal data from Sully Vent (dive 3238). A) The spectra from sixty 30 second exposure images are compared to the calculated flux for a theoretical black body of temperature $340 \pm 10^{\circ} \mathrm{C}$ and emissivity $0.3 \pm 0.1$ at a distance of $50 \pm 5 \mathrm{~cm}$ from the ALISS camera. B) Time series of the data plotted in (A). Data are normalized by the mean flux per band and offset for ease of viewing. The shift in the 450,550 , and $599 \mathrm{~nm}$ bands at 15 minutes is on the order of 50 photons $/ \mathrm{sec} / \mathrm{cm}^{2}$.

Figure 5.20 Processed ALISS images of $P$ Vent taken with both filter arrays on dive 3182. The layout of the filters is given in Figure 5.4.

Figure 5.21 Spatial distribution of light at $P$ Vent (dive 3182). A) Image of $P$ Vent taken under artificial illumination in the $550 \mathrm{~nm}$ band. B) Image of ambient light emission from $\mathrm{P}$ Vent in the $870 \mathrm{~nm}$ band. C) Portions of the source mask isolated for spectral analysis. Note that 300 pixels corresponds to $\sim 15 \mathrm{~cm}$. 
Figure 5.22 Spectral data from $P$ Vent (dive 3182 ). Data from the $100 \mathrm{~nm}$ bandwidth filters are plotted for each of the regions shown in Figure 5.21C. The data are compared to the calculated flux for a theoretical black body of temperature $345 \pm 10^{\circ} \mathrm{C}$ and emissivity $0.3 \pm 0.1$ at a distance of $50 \pm 5 \mathrm{~cm}$ from the ALISS camera. The 0-25 percentile pixels correspond to a temperature of $265^{\circ} \mathrm{C}$. The drop-off in flux above $870 \mathrm{~nm}$ is due to the high attenuation of light in seawater at those wavelengths.

Figure 5.23 Processed ALISS images of V Vent taken with both filter arrays on dive 3186. The layout of the filters is given in Figure 5.4.

Figure 5.24 Spatial distribution of light at V Vent (dive 3186). A) Image of V Vent taken under artificial illumination in the $550 \mathrm{~nm}$ band. B) Image of ambient light emission from V Vent in the $870 \mathrm{~nm}$ band. C) Portions of the source masks isolated for spectral analysis. (Each vent was analyzed individually). Note that 300 pixels corresponds to $\sim 15 \mathrm{~cm}$.

Figure 5.25 Spectral data from V Vent (dive 3186). Data from the $100 \mathrm{~nm}$ bandwidth filters are plotted for each of the regions shown in Figures 5.24C. The data for the center vent (A) are compared to the calculated flux for a theoretical black body of temperature $265 \pm 10^{\circ} \mathrm{C}$ and emissivity $0.3 \pm 0.1$ at a distance of $50 \pm 5 \mathrm{~cm}$ from the ALISS camera. The data for the right vent (B) are compared to a $275^{\circ} \mathrm{C}$ black body with the same uncertainties. The drop-off in flux above $870 \mathrm{~nm}$ is due to the high attenuation of light in seawater at those wavelengths. 
Figure 5.26 Processed ALISS images of L Vent (AdV 4-9) taken with both filter arrays. The layout of the filters is given in Figure 5.4.

Figure 5.27 Spatial distribution of light at L Vent (dive 3191). A) Image of L Vent taken under artificial illumination in the $550 \mathrm{~nm}$ band. B) Image of ambient light emission from $\mathrm{L}$ Vent in the $870 \mathrm{~nm}$ band. C) Portions of the source masks isolated for spectral analysis. Note that 300 pixels corresponds to $\sim 15 \mathrm{~cm}$.

Figure 5.28 Spectral data from $L$ Vent (dive 3191). Data from the $100 \mathrm{~nm}$ bandwidth filters are plotted for each of the regions shown in Figure 5.27C. The data are compared to the calculated flux for a theoretical black body of temperature $295 \pm 10^{\circ} \mathrm{C}$ and emissivity $0.3 \pm 0.1$ at a distance of $50 \pm 5 \mathrm{~cm}$ from the ALISS camera. The $0-25$ percentile pixels correspond to a temperature of $260^{\circ} \mathrm{C}$. The drop-off in flux above $870 \mathrm{~nm}$ is due to the high attenuation of light in seawater at those wavelengths.

Figure 5.29 Spatial distribution of light at Q Vent (dive 3187). A) Image of Q Vent taken under artificial illumination in the $550 \mathrm{~nm}$ band. B) Image of ambient light emission from Q Vent in the $870 \mathrm{~nm}$ band. C) Portions of the source masks isolated for spectral analysis. Note that 300 pixels corresponds to $\sim 15 \mathrm{~cm}$. 
Figure 5.30 Spectral data from Q Vent (dive 3187). Data from the $100 \mathrm{~nm}$ bandwidth filters are plotted for each of the regions shown in Figure 5.29C. The data are compared to the calculated flux for a theoretical black body of temperature $320 \pm 10^{\circ} \mathrm{C}$ and emissivity $0.3 \pm 0.1$ at a distance of $50 \pm 5 \mathrm{~cm}$ from the ALISS camera. The drop-off in flux above $870 \mathrm{~nm}$ is due to the high attenuation of light in seawater at those wavelengths. The 0-25 percentile pixels correspond to a temperature of $270^{\circ} \mathrm{C}$.

Figure 5.31 Spatial distribution of light from a beehive at Q Vent (dive 3174). A) Image of the beehive taken under artificial illumination in the $550 \mathrm{~nm}$ band. B) Image of ambient light emission from the beehive in the $870 \mathrm{~nm}$ band. C) Spectral analyses were performed on the light from the beehive structure, and the fluid layer on the side of the beehive. Note that 300 pixels corresponds to $\sim 15 \mathrm{~cm}$.

Figure 5.32 Spectral data from the beehive at Q Vent (dive 3174). Data from the $100 \mathrm{~nm}$ bandwidth filters are plotted for each of the regions shown in Figure 5.31C. The data are compared to the calculated flux for a theoretical black body of temperature $265 \pm 10^{\circ} \mathrm{C}$ and emissivity $0.9 \pm 0.1$ at a distance of $50 \pm 5 \mathrm{~cm}$ from the ALISS camera. The drop-off in flux above $870 \mathrm{~nm}$ is due to the high attenuation of light in seawater at those wavelengths. 


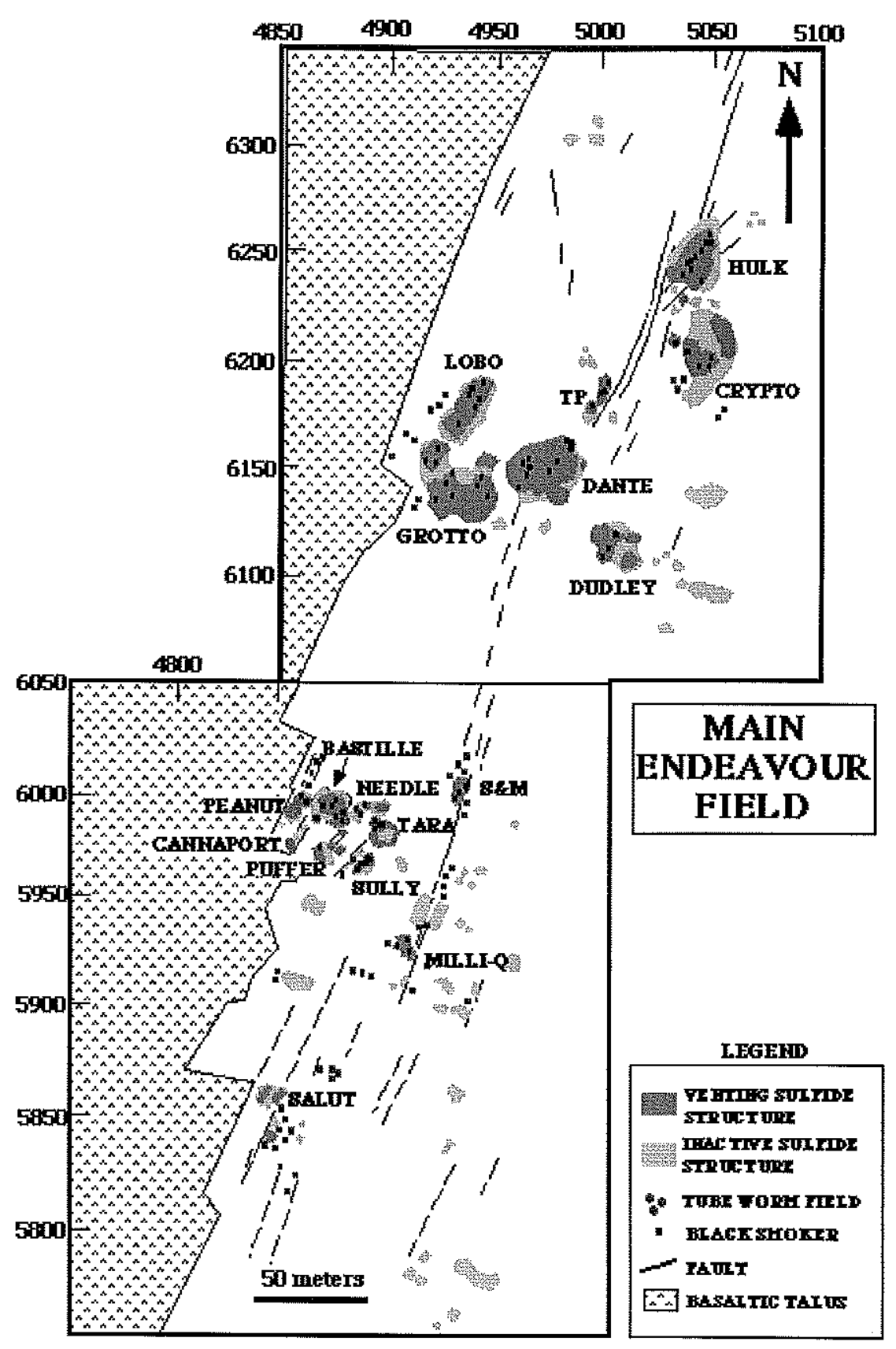

Figure 5.1 
A

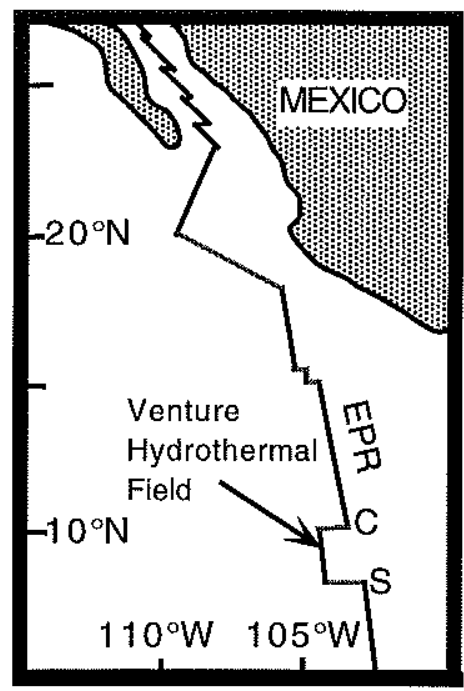

B

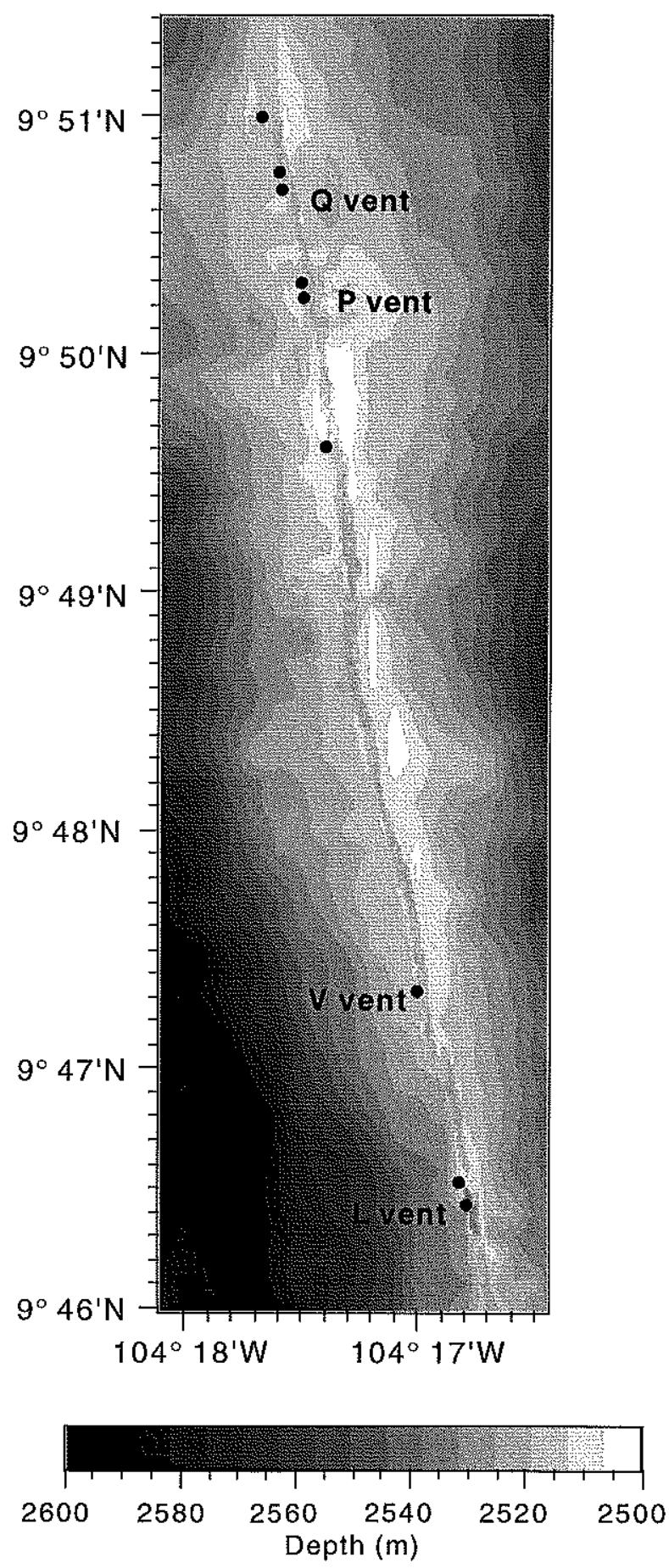

Figure 5.2 
A

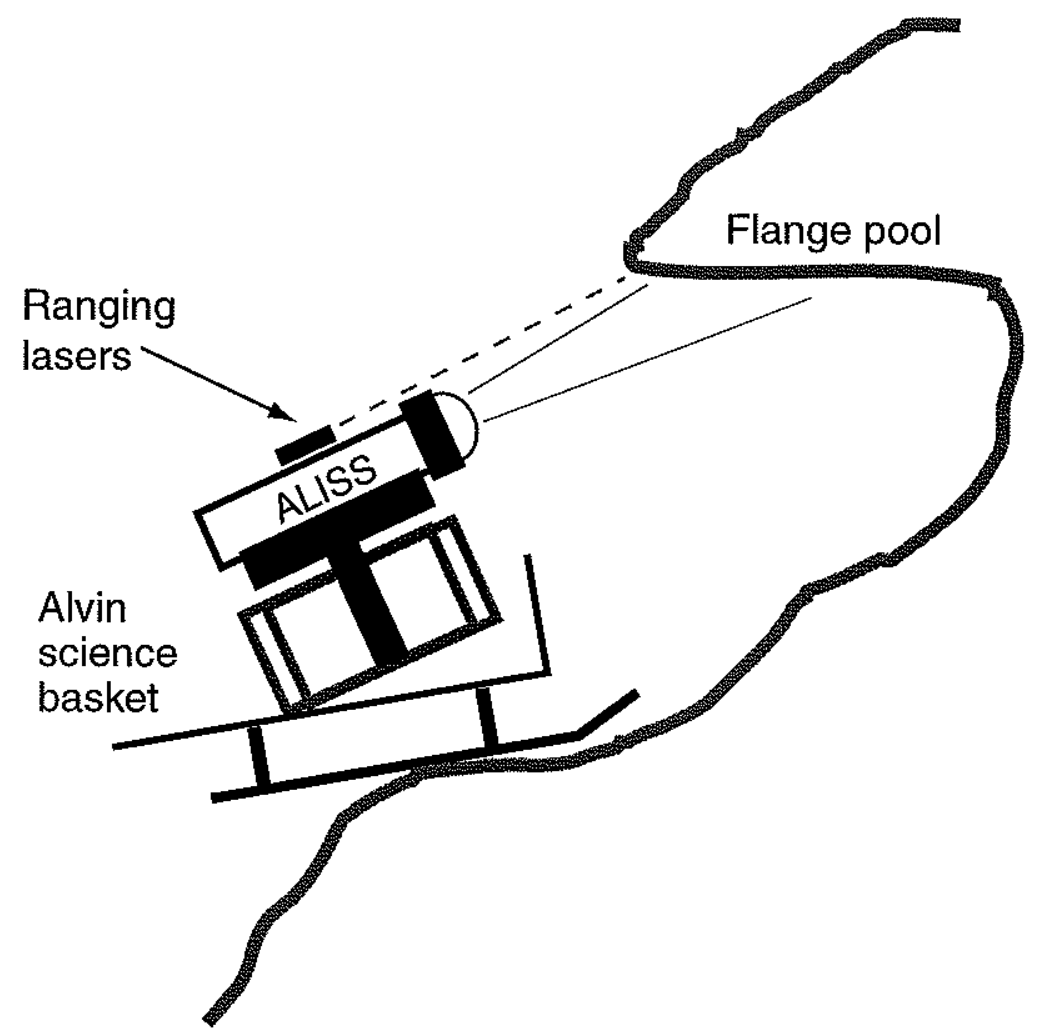

B

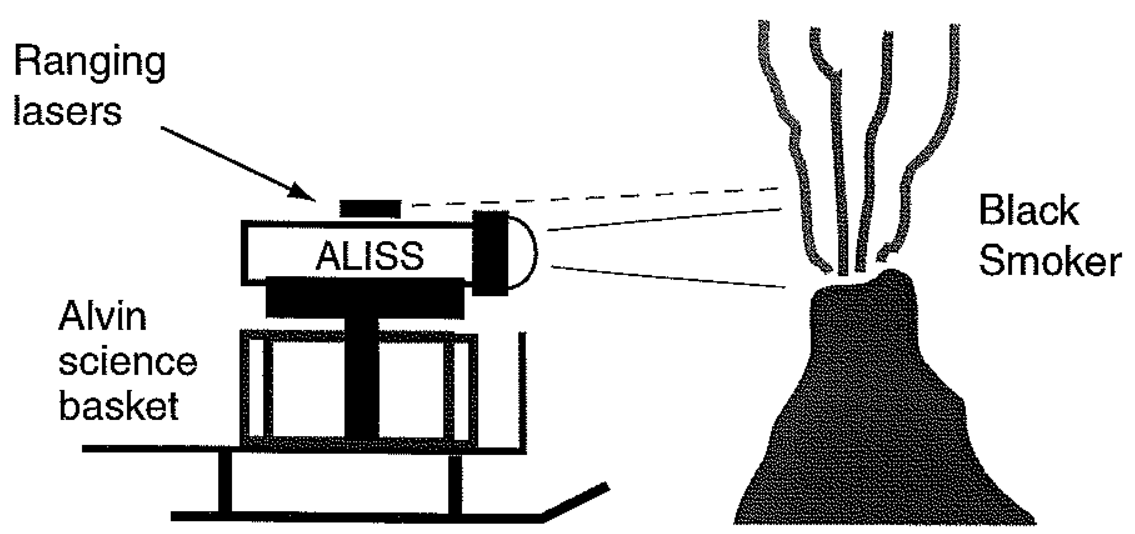

Figure 5.3 
Filter Array \#1

\begin{tabular}{|c|c|c|}
\hline $\begin{array}{c}599 \mathrm{~nm} \\
(100)\end{array}$ & $\begin{array}{c}550 \mathrm{~nm} \\
(100)\end{array}$ & $\begin{array}{c}450 \mathrm{~nm} \\
(80)\end{array}$ \\
\hline $\begin{array}{c}753 \mathrm{~nm} \\
(108)\end{array}$ & $\begin{array}{c}705 \mathrm{~nm} \\
(96)\end{array}$ & $\begin{array}{c}652 \mathrm{~nm} \\
(104)\end{array}$ \\
\hline $\begin{array}{c}947 \mathrm{~nm} \\
(80)\end{array}$ & $\begin{array}{c}870 \mathrm{~nm} \\
(140)\end{array}$ & $\begin{array}{c}792 \mathrm{~nm} \\
(102)\end{array}$ \\
\hline
\end{tabular}

Filter Array \#2

\begin{tabular}{|c|c|c|}
\hline $\begin{array}{c}576 \mathrm{~nm} \\
(49)\end{array}$ & $\begin{array}{c}497 \mathrm{~nm} \\
(91)\end{array}$ & $\begin{array}{c}475 \mathrm{~nm} \\
(52)\end{array}$ \\
\hline $\begin{array}{c}780 \mathrm{~nm} \\
(50)\end{array}$ & $\begin{array}{c}676 \mathrm{~nm} \\
(49)\end{array}$ & $\begin{array}{c}589 \mathrm{~nm} \\
(10)\end{array}$ \\
\hline $\begin{array}{c}980 \mathrm{~nm} \\
(65)\end{array}$ & $\begin{array}{c}902 \mathrm{~nm} \\
(130)\end{array}$ & $\begin{array}{c}878 \mathrm{~nm} \\
(68)\end{array}$ \\
\hline
\end{tabular}

Figure 5.4 
Filter Array \#1

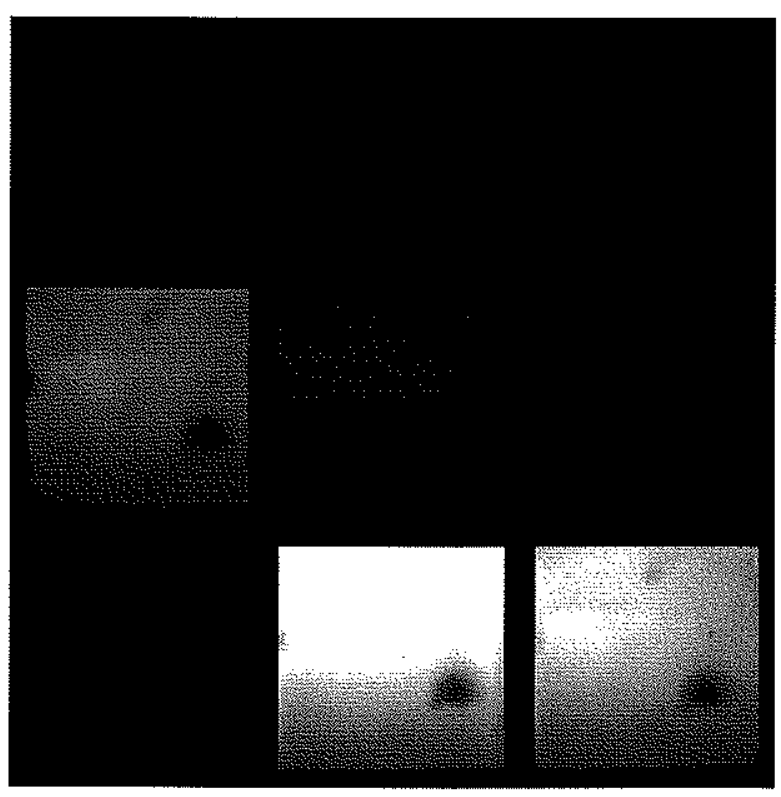

Filter Array \#2

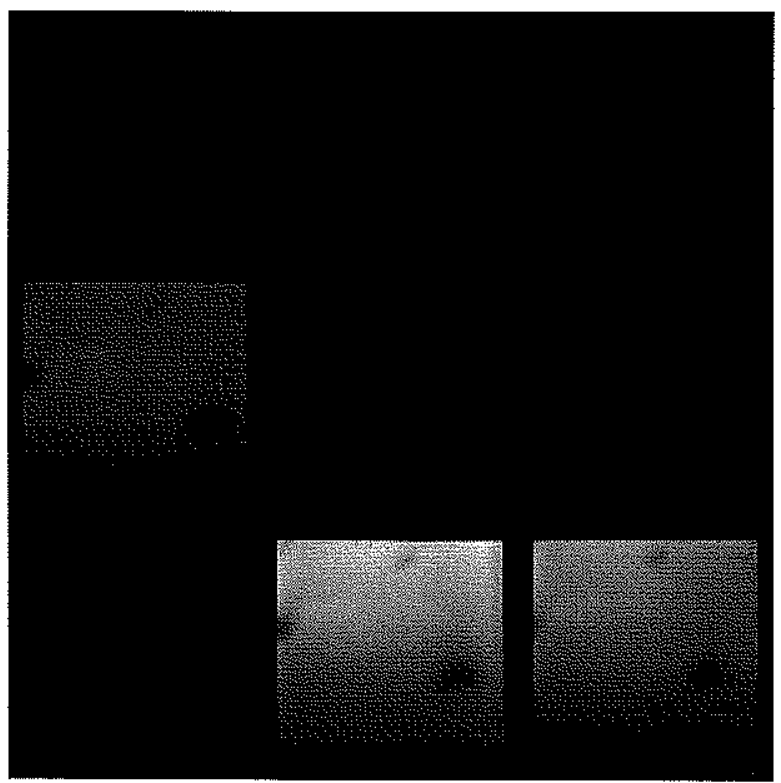

Figure 5.5 


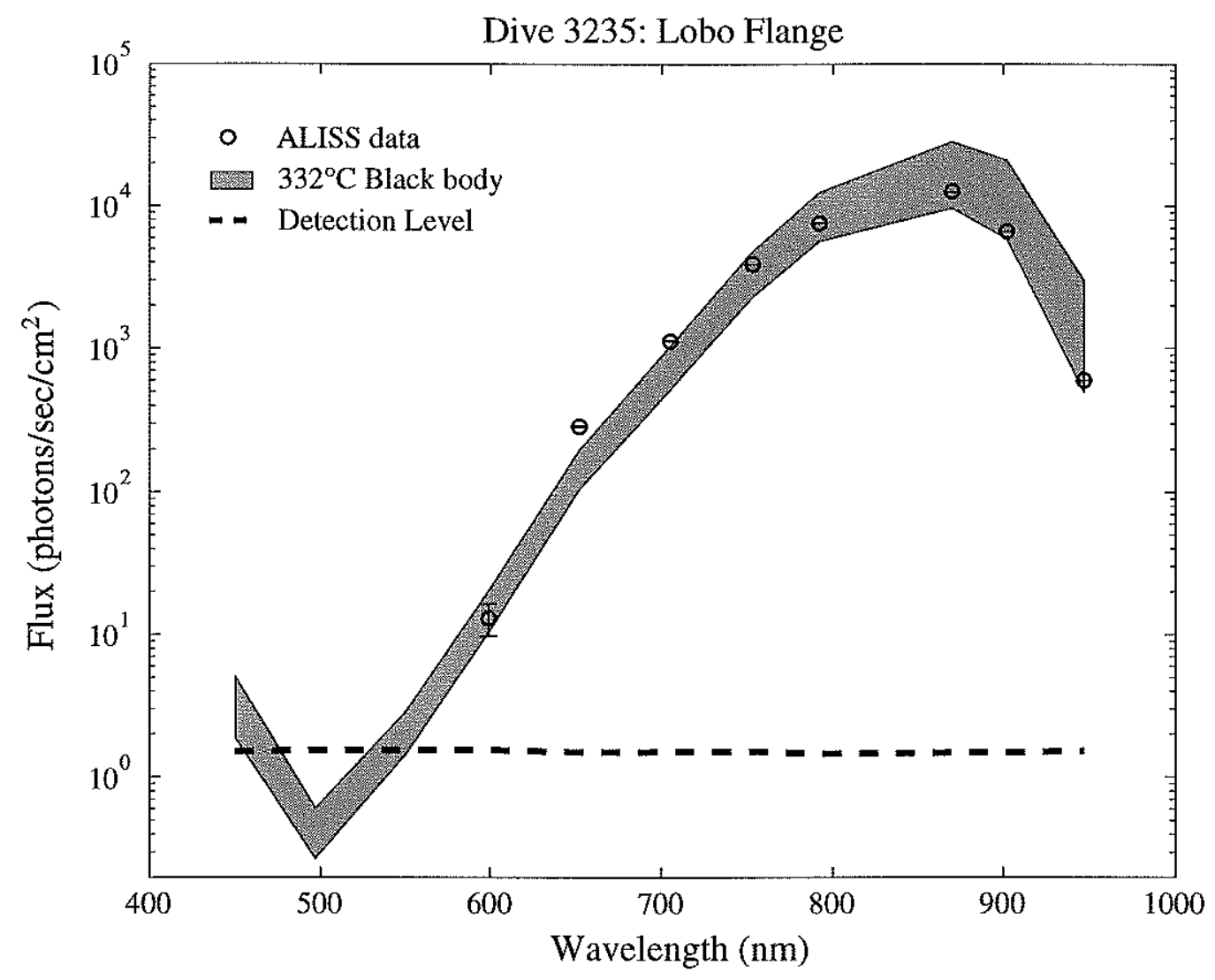

Figure 5.6 
Filter Array \#1

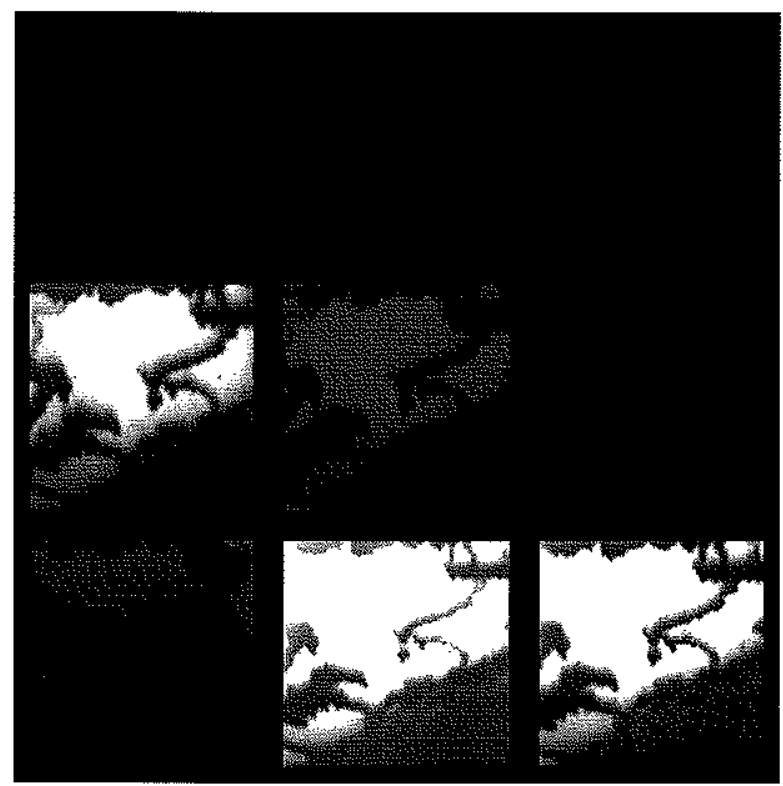

Filter Array \#2

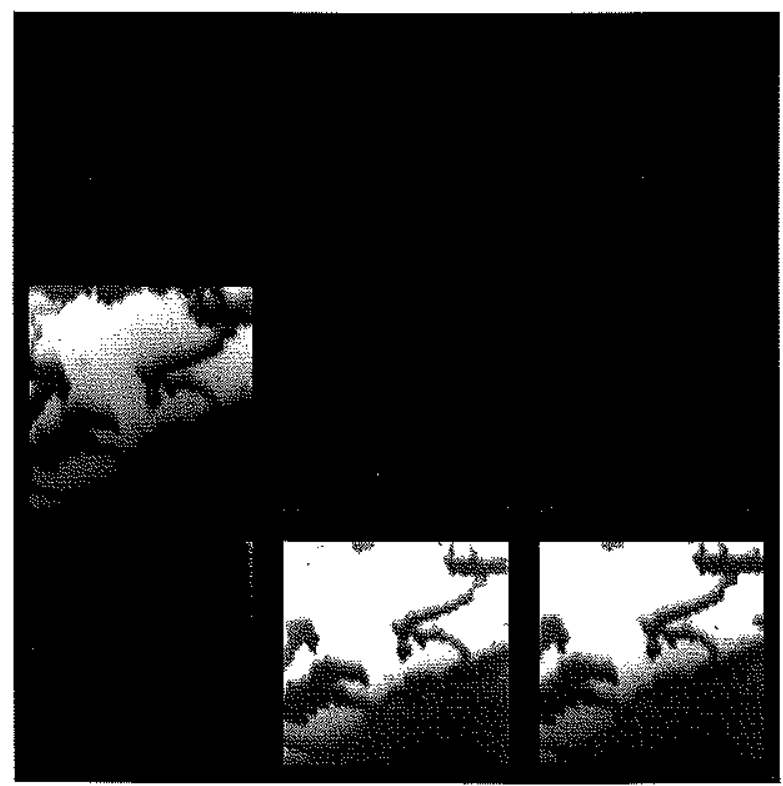

Figure 5.7 


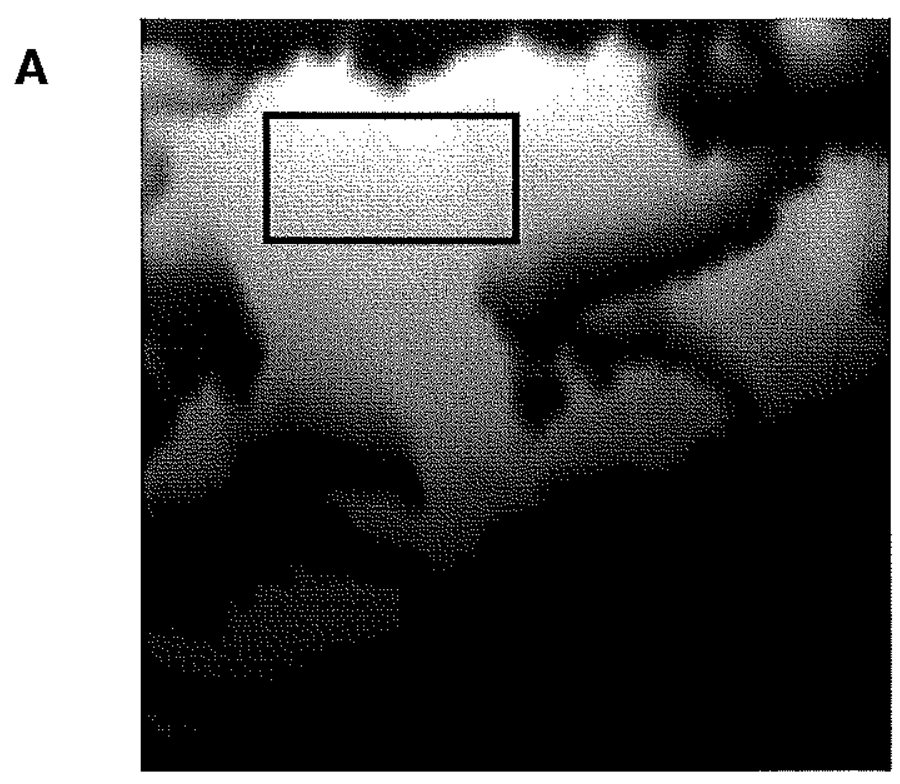

B

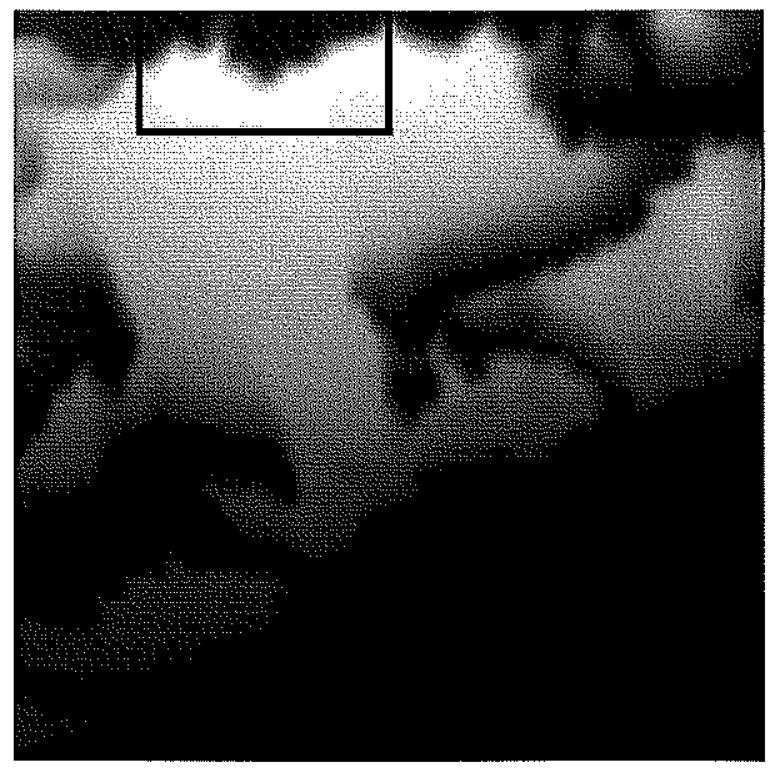

Figure 5.8 

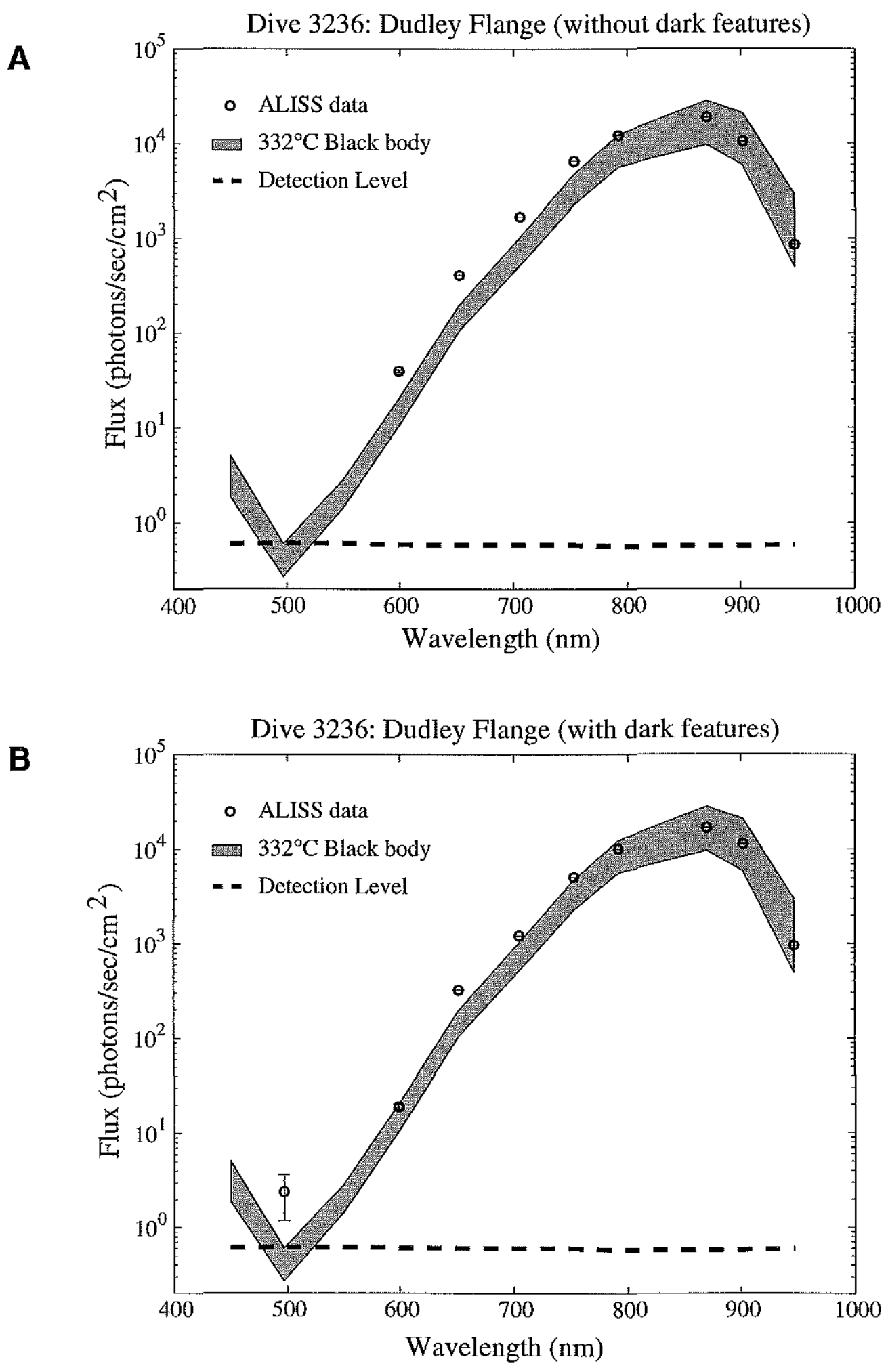

Figure 5.9 


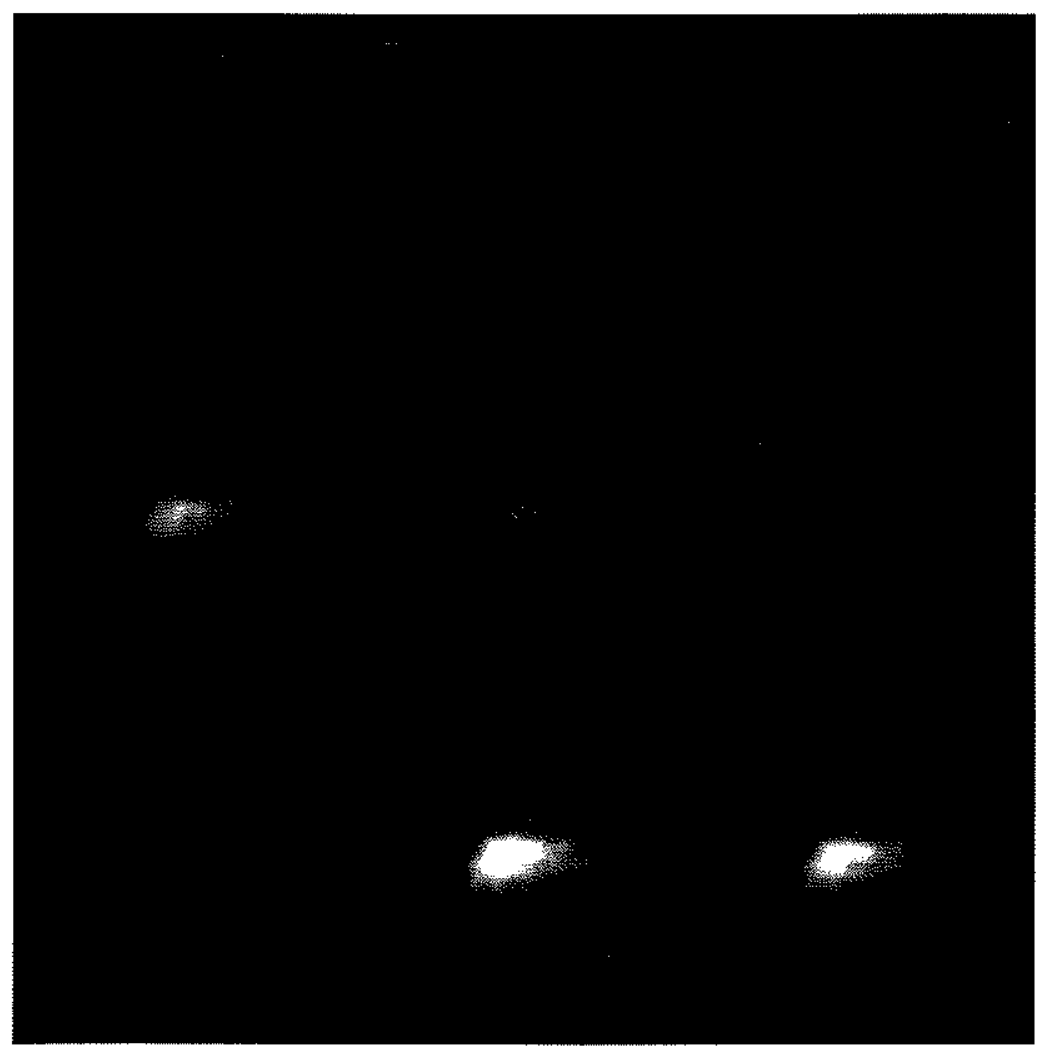

Figure 5.10 
A

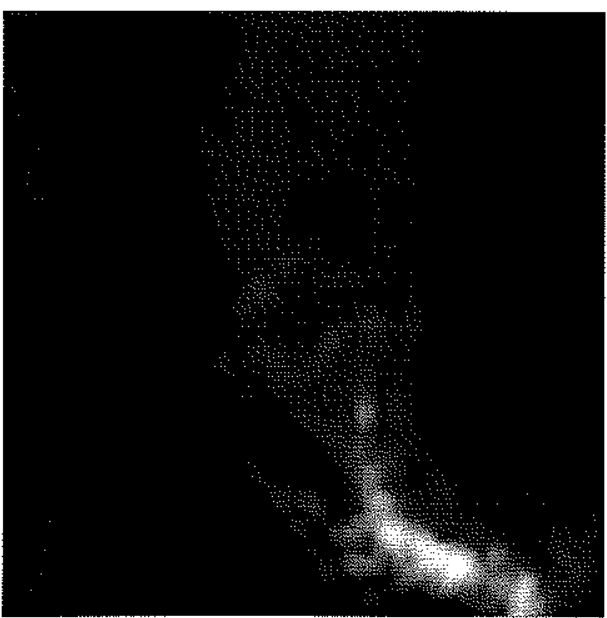

B

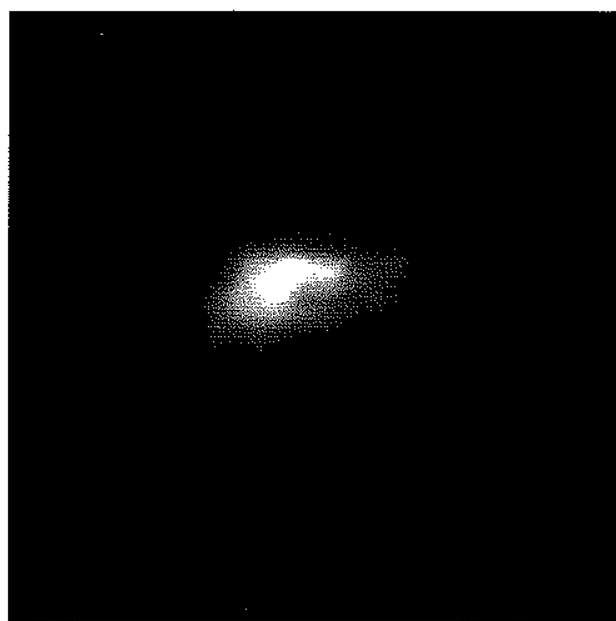

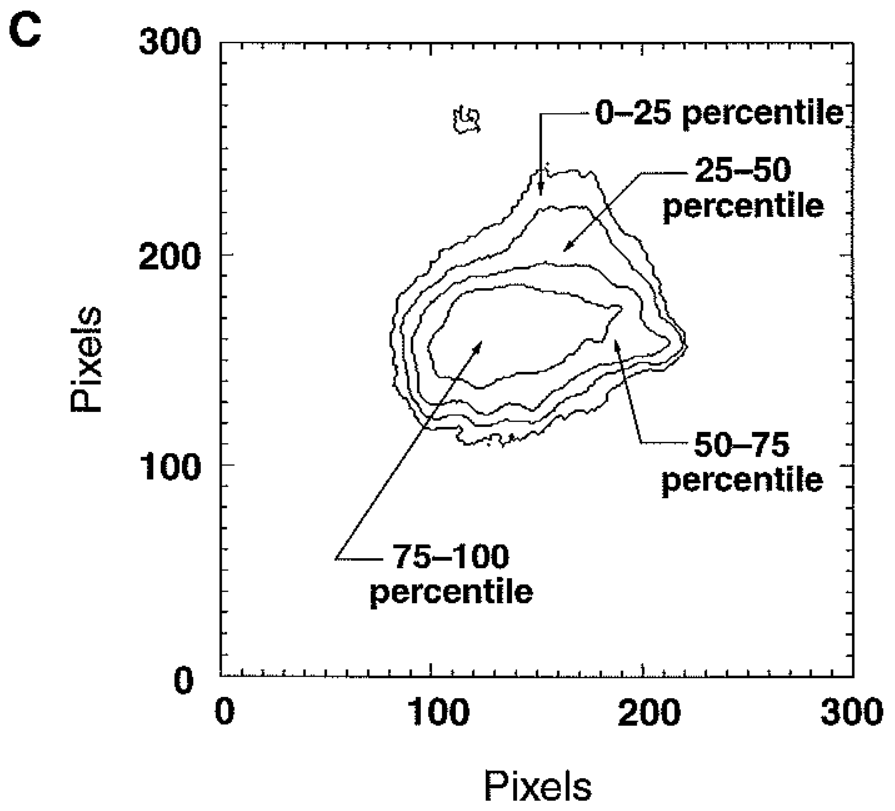

Figure 5.11 

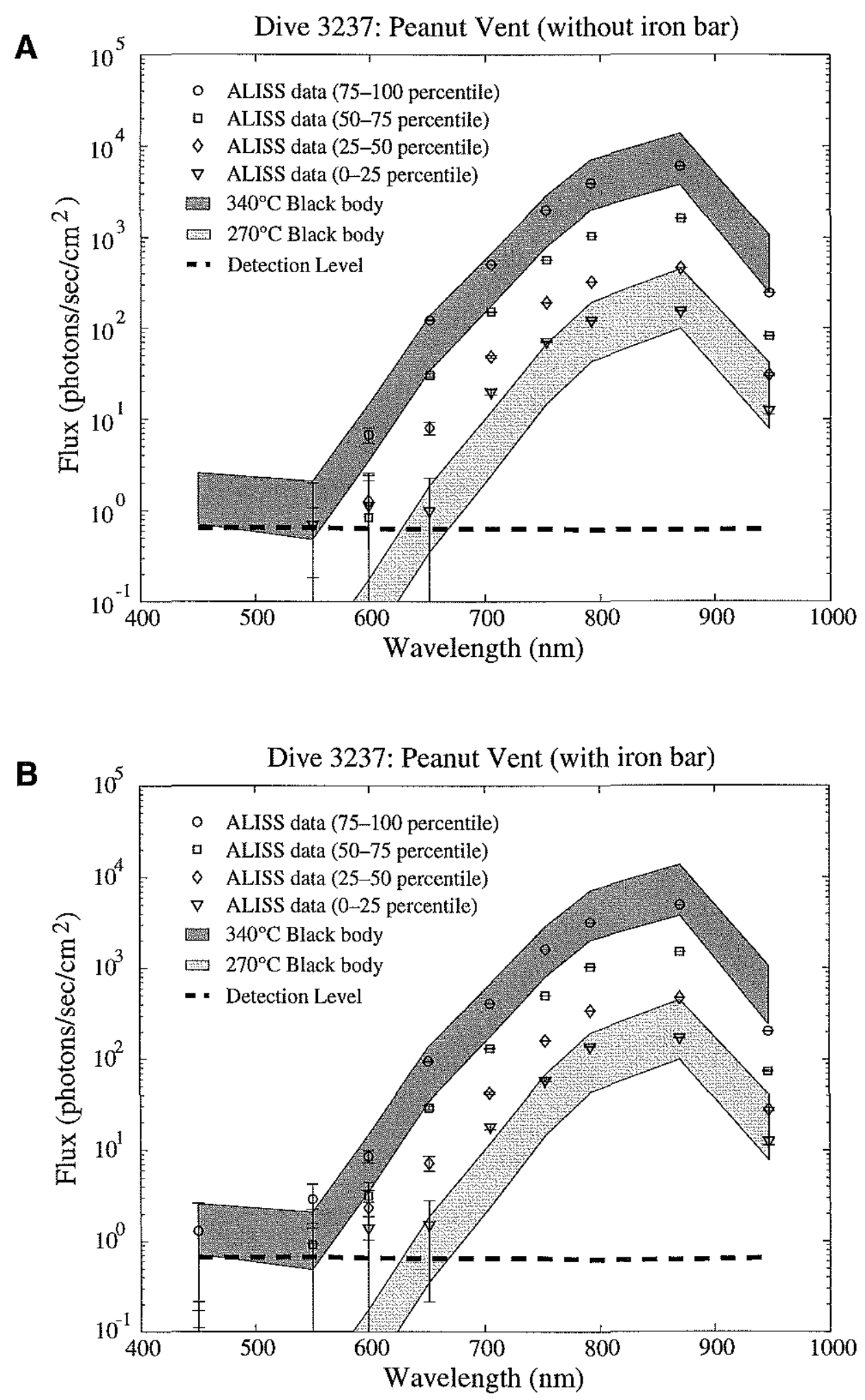

Figure 5.12 


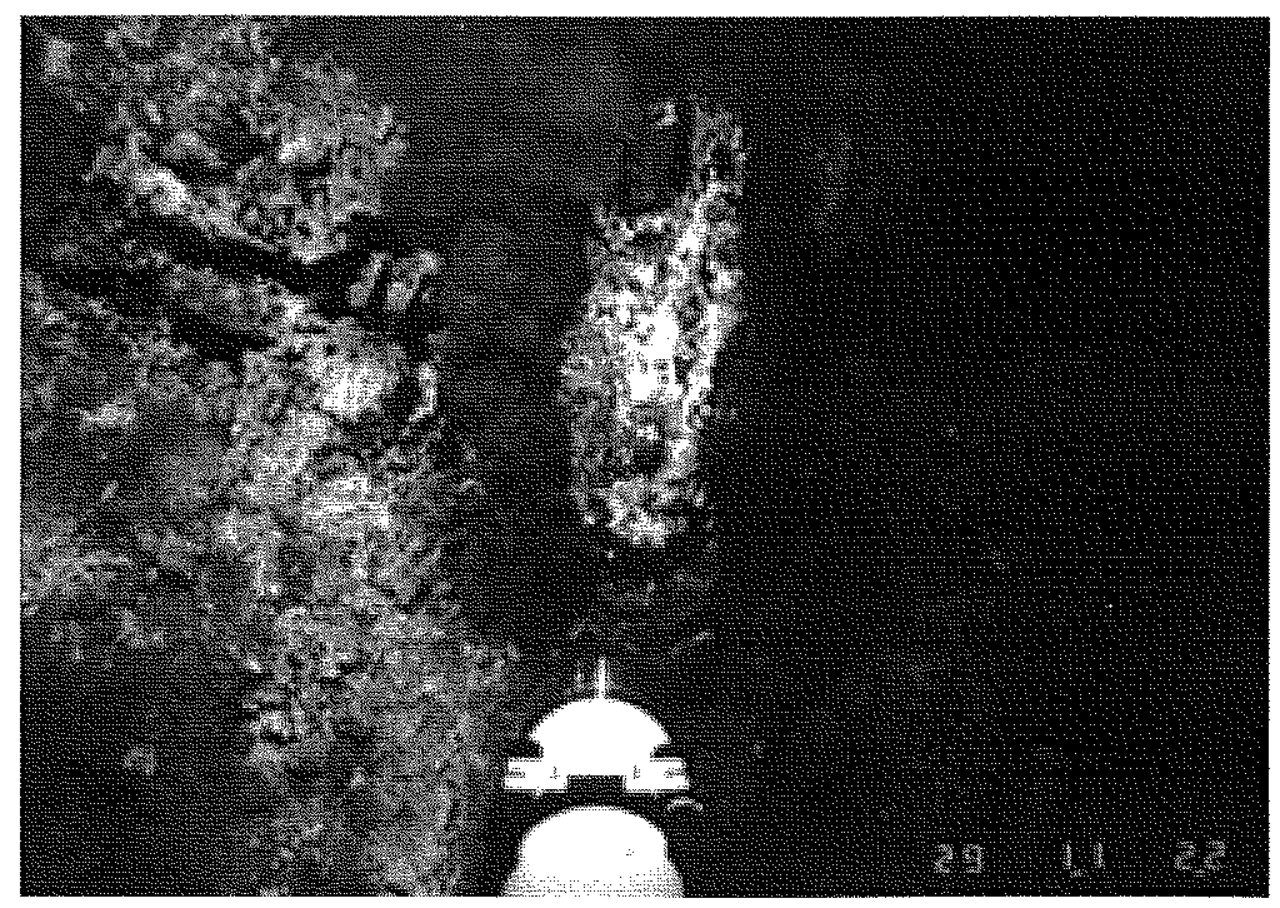

Figure 5.13 
A

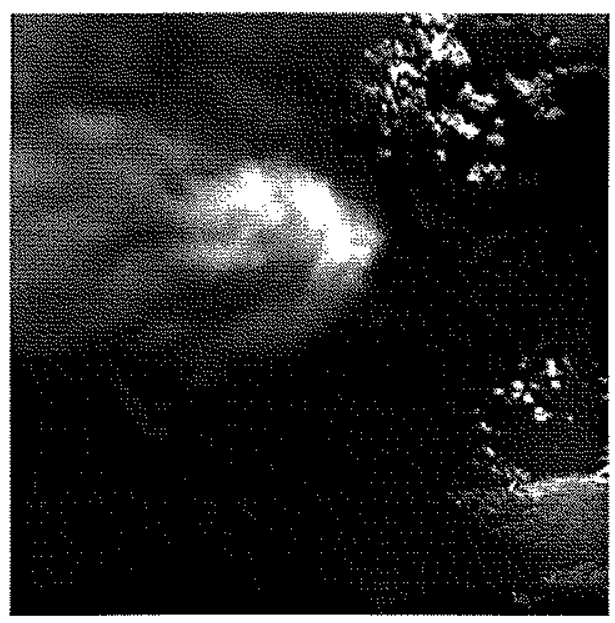

B

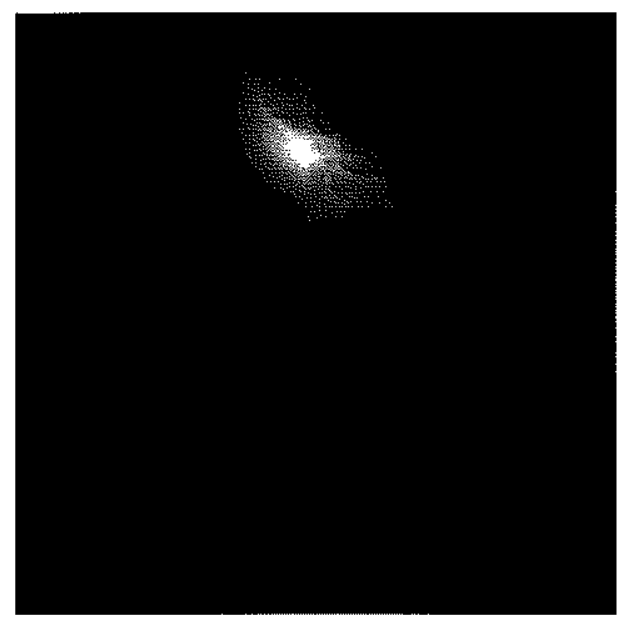

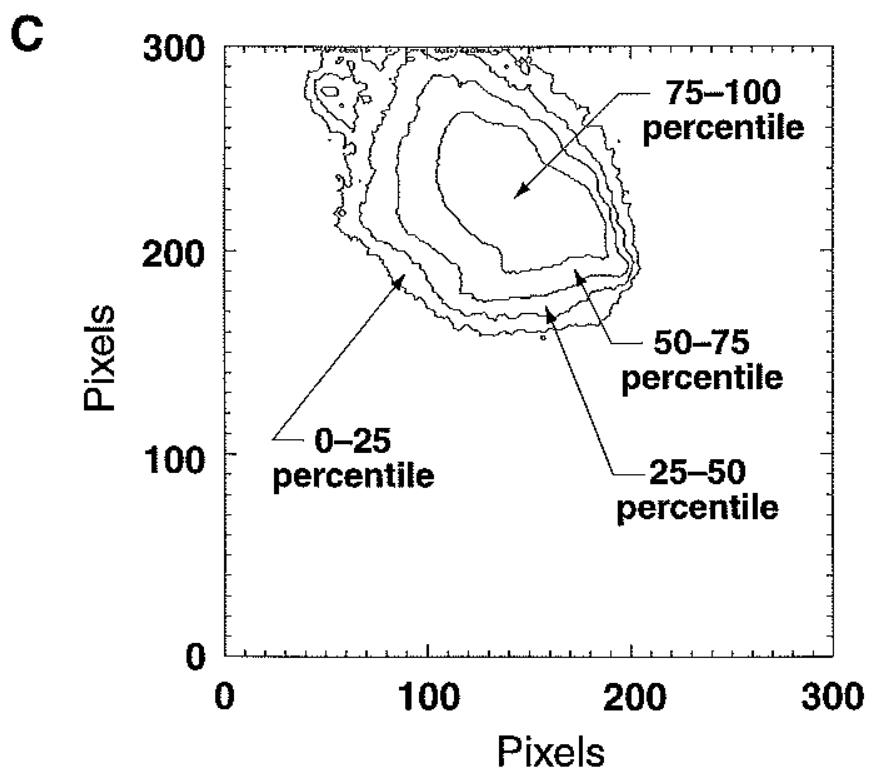

Figure 5.14 
A
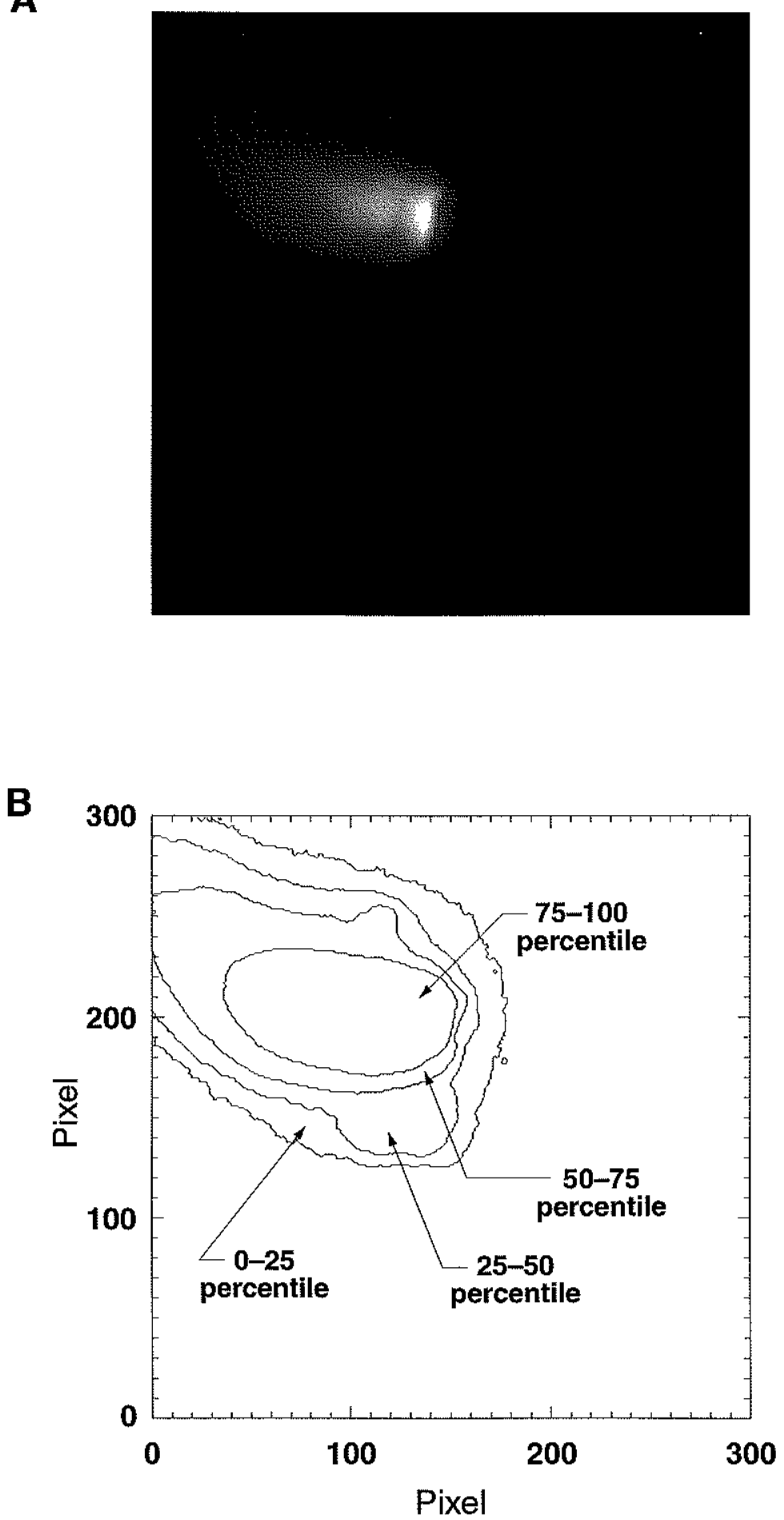

Figure 5.15 

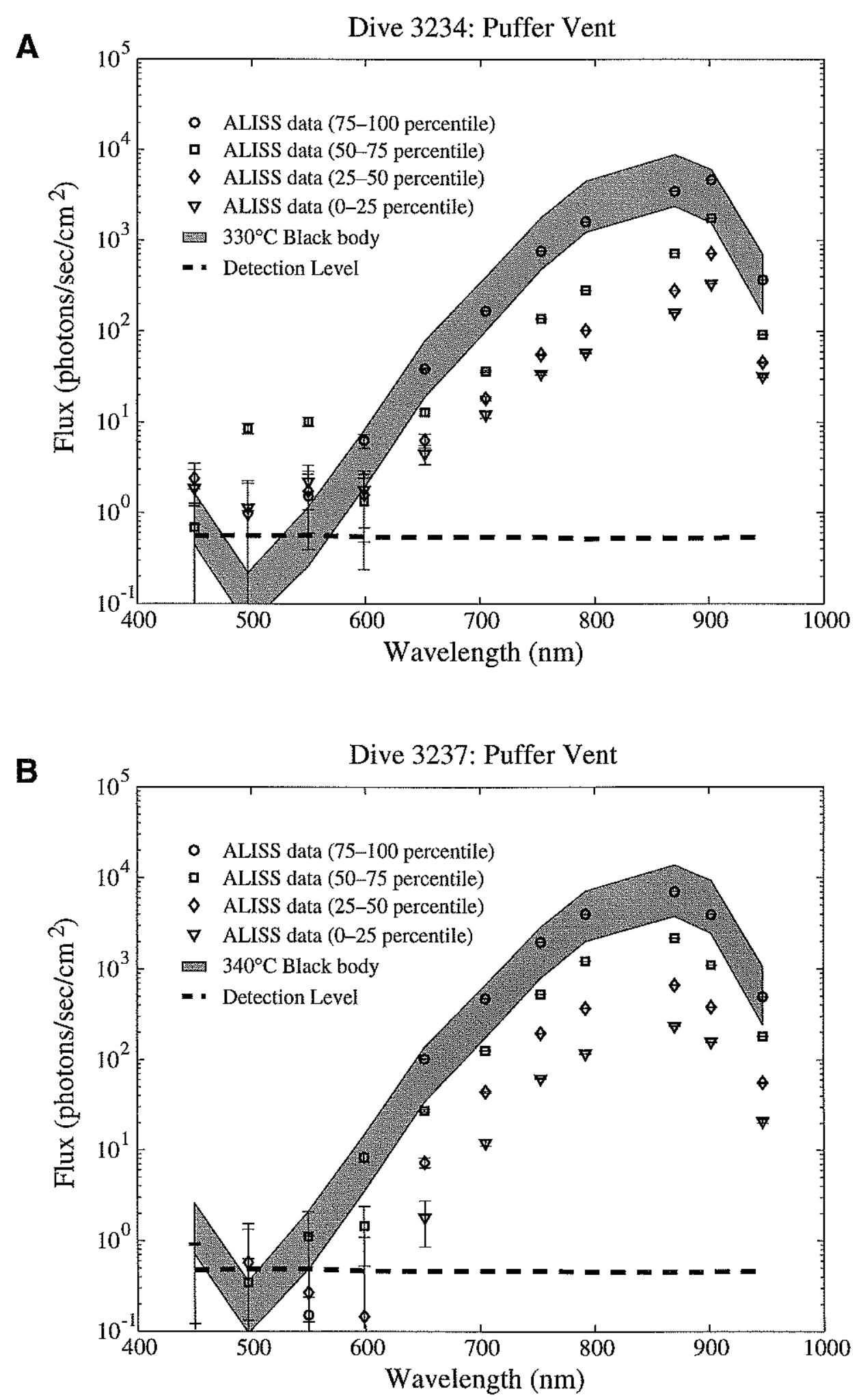

Figure 5.16 
A

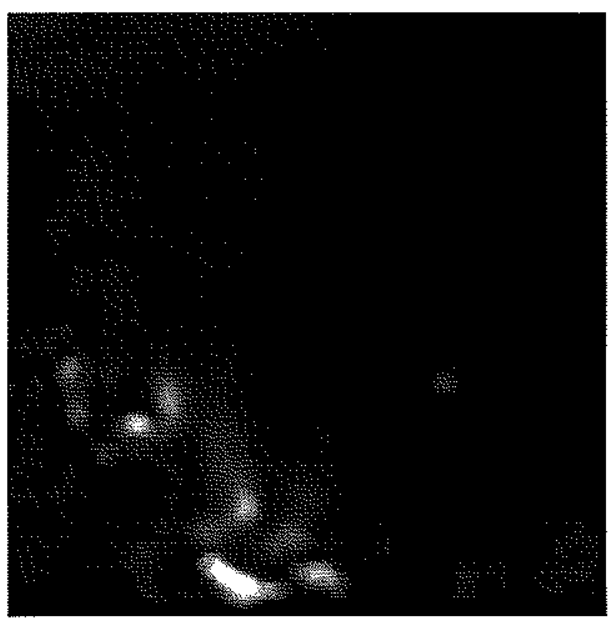

B

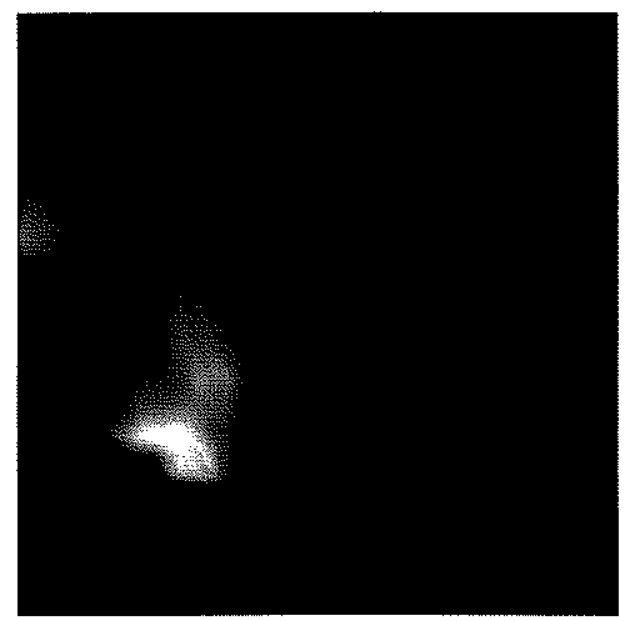

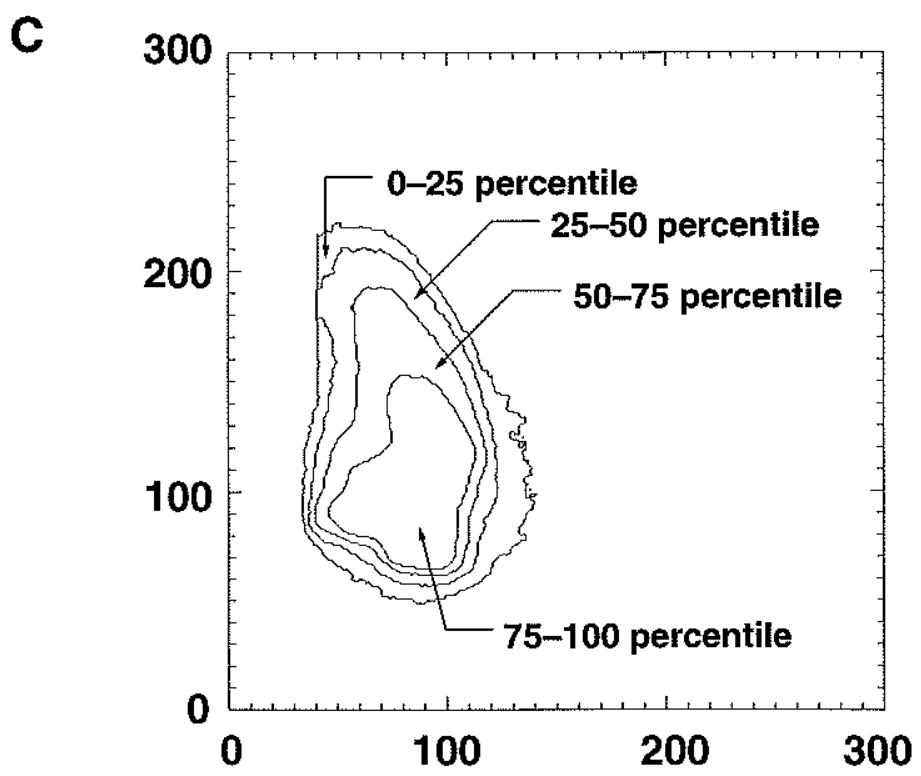

Figure 5.17 
Dive 3238: Sully Vent

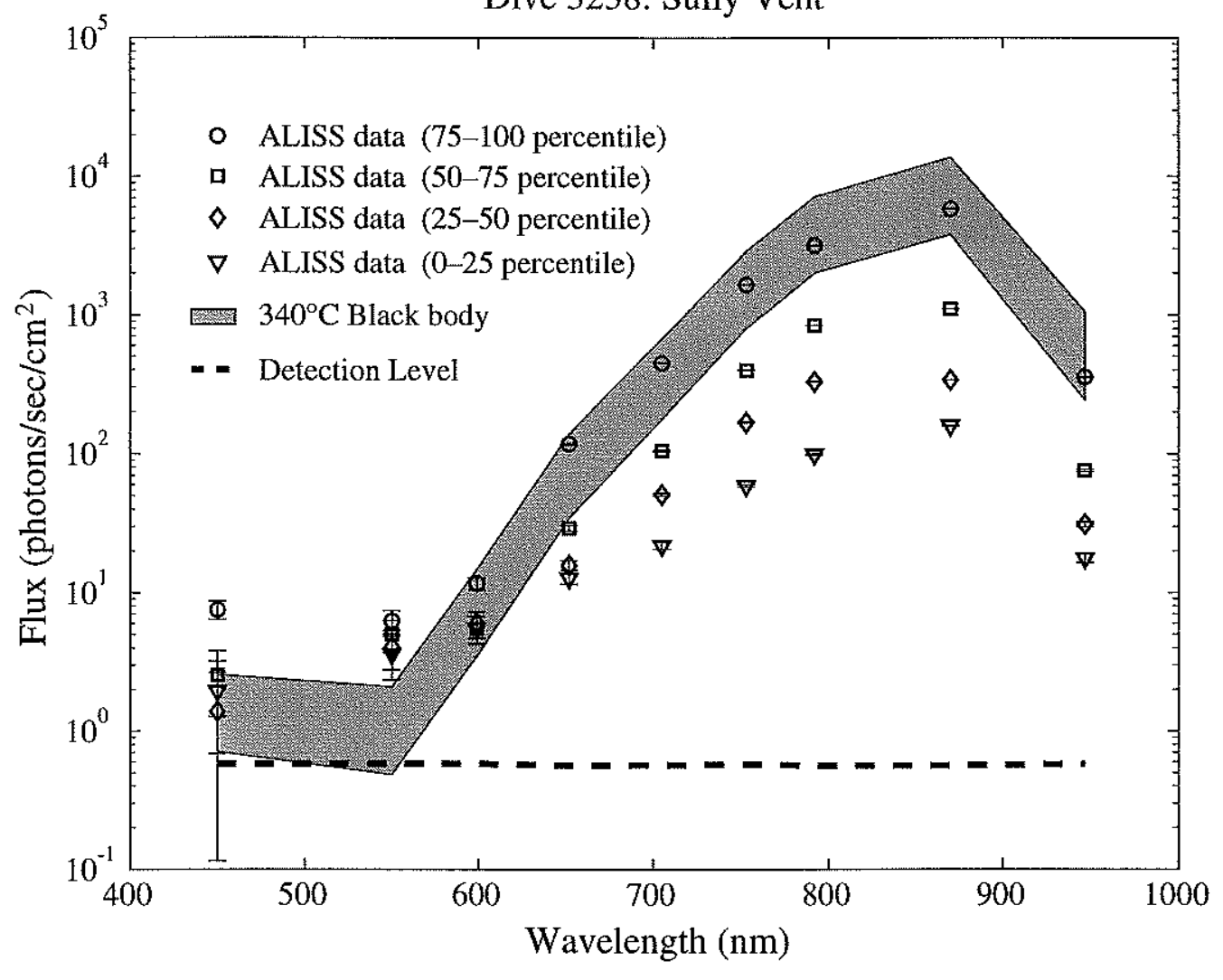

Figure 5.18 


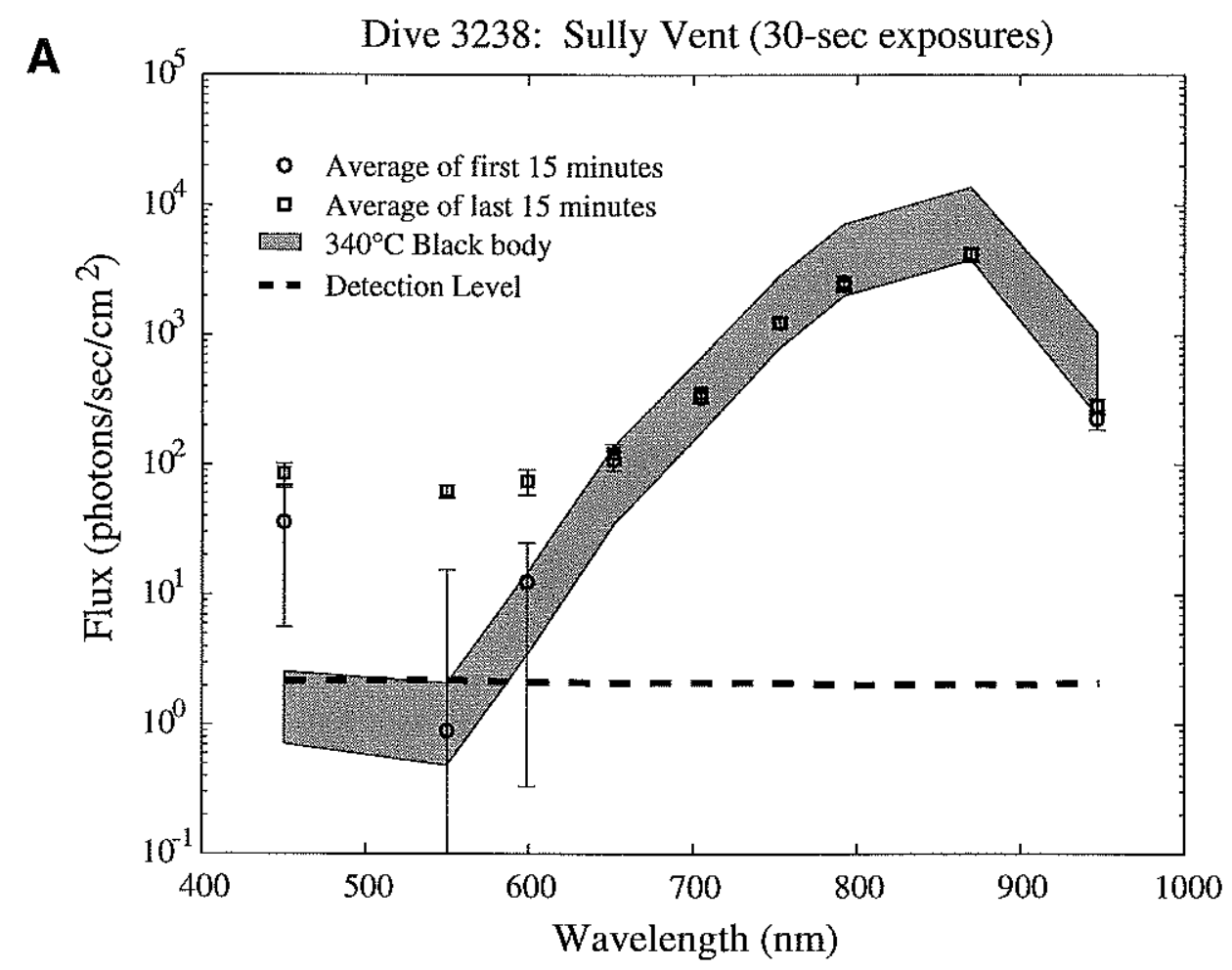

B

Dive 3238: Sully Vent (30-sec exposures)

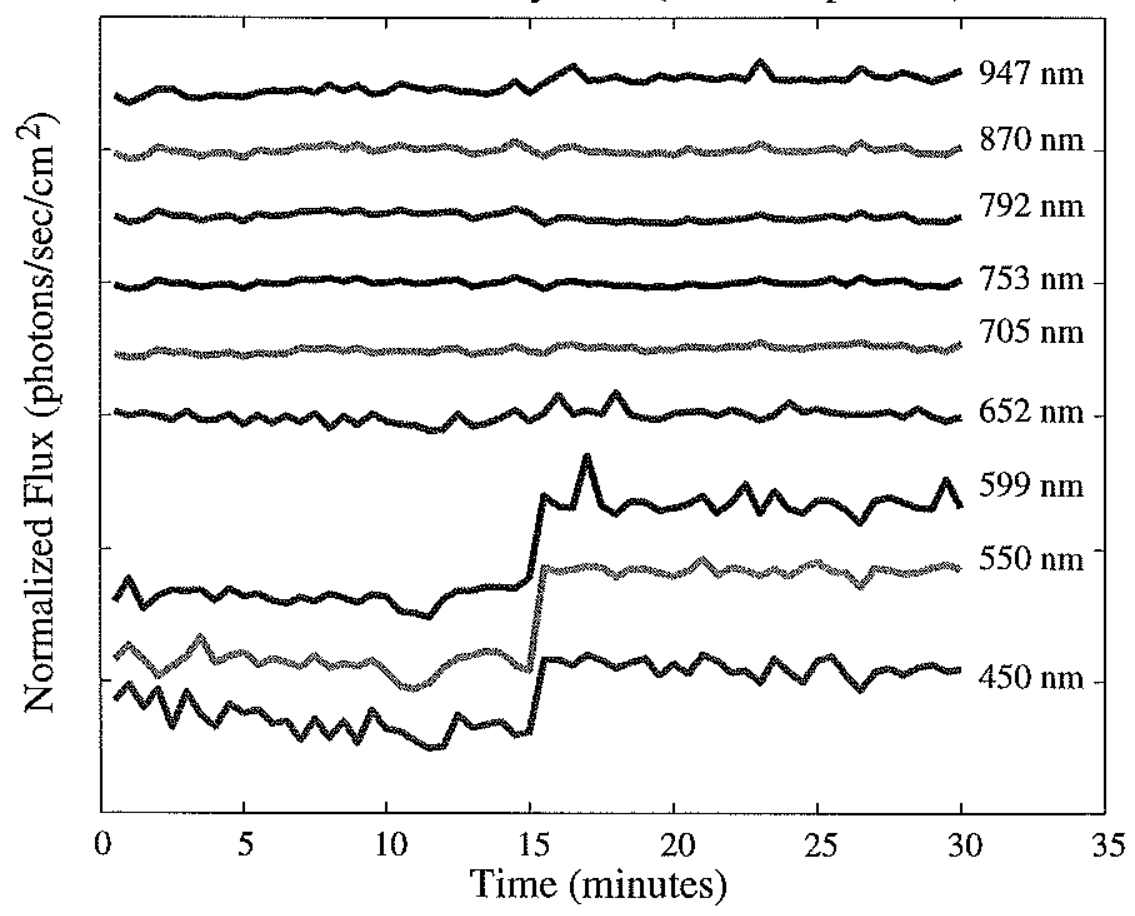

Figure 5.19 
Filter Array \#1

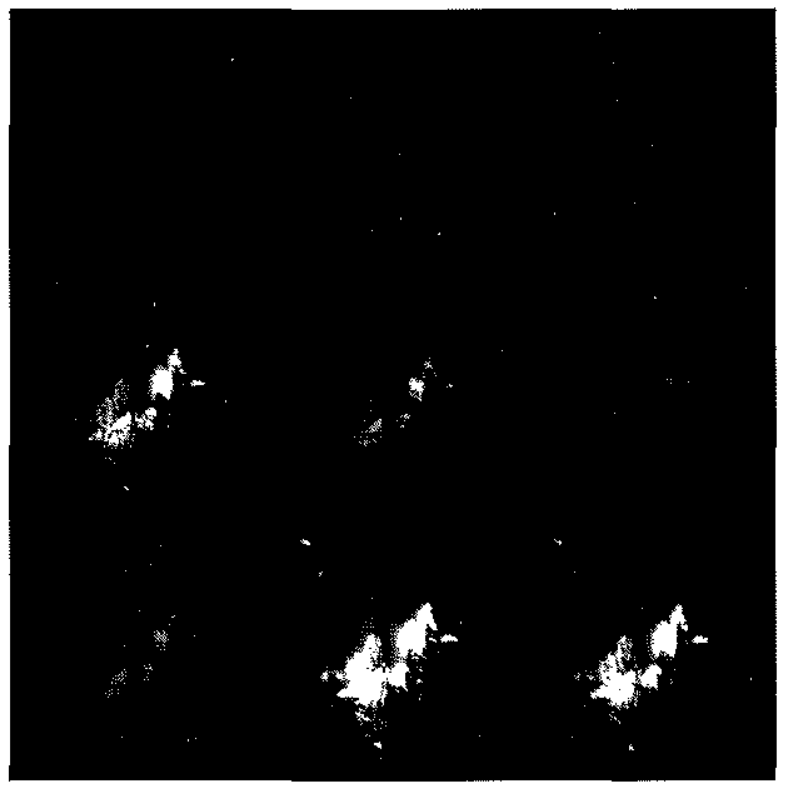

Filter Array \#2

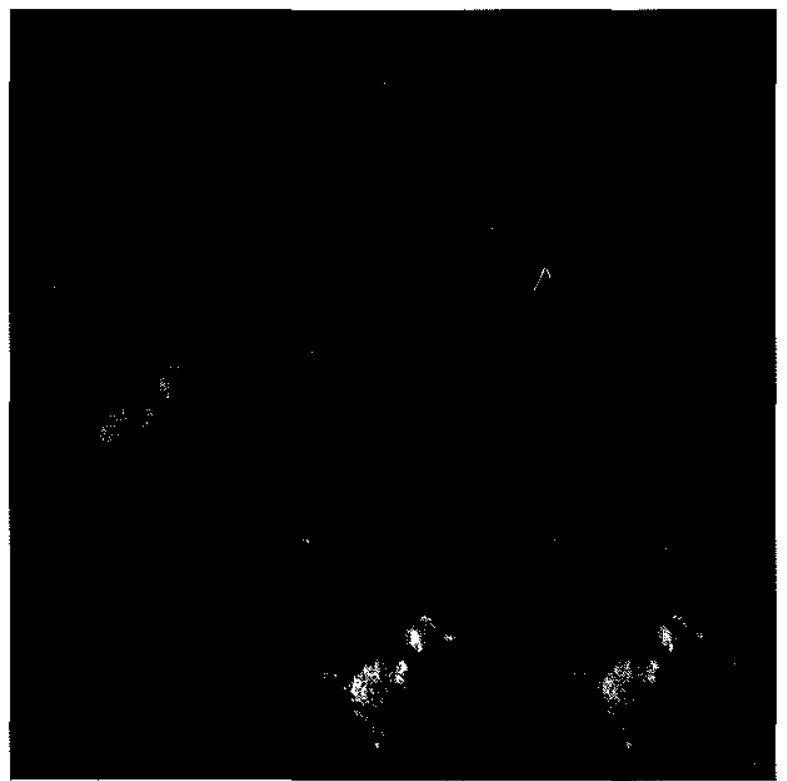

Figure 5.20 
A

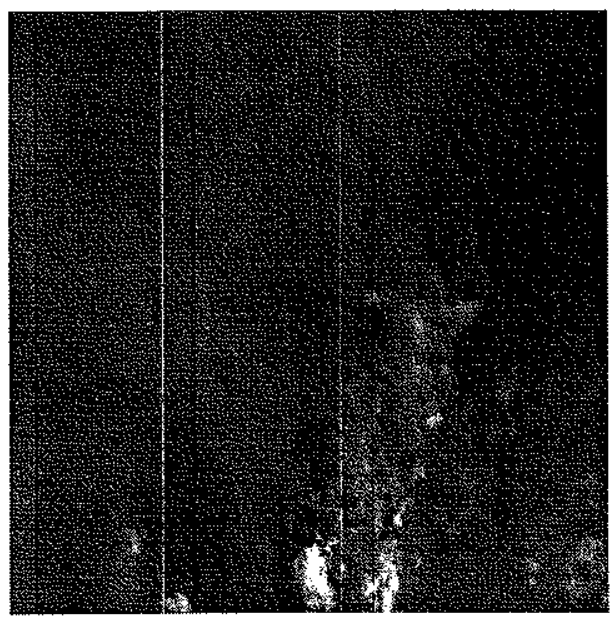

B

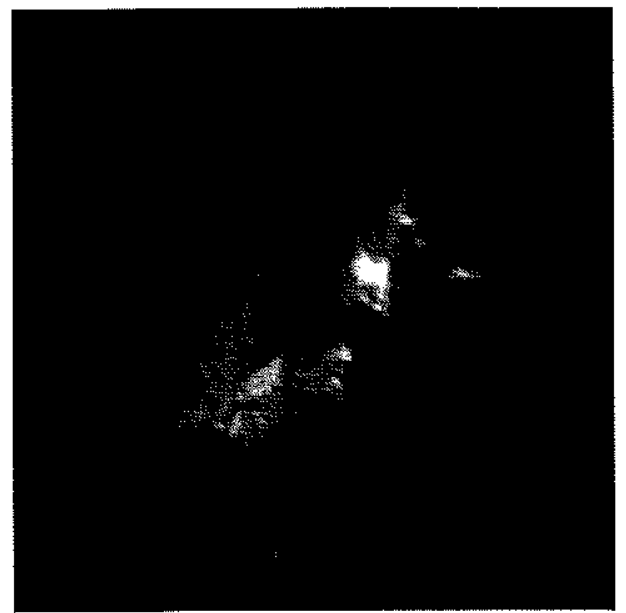

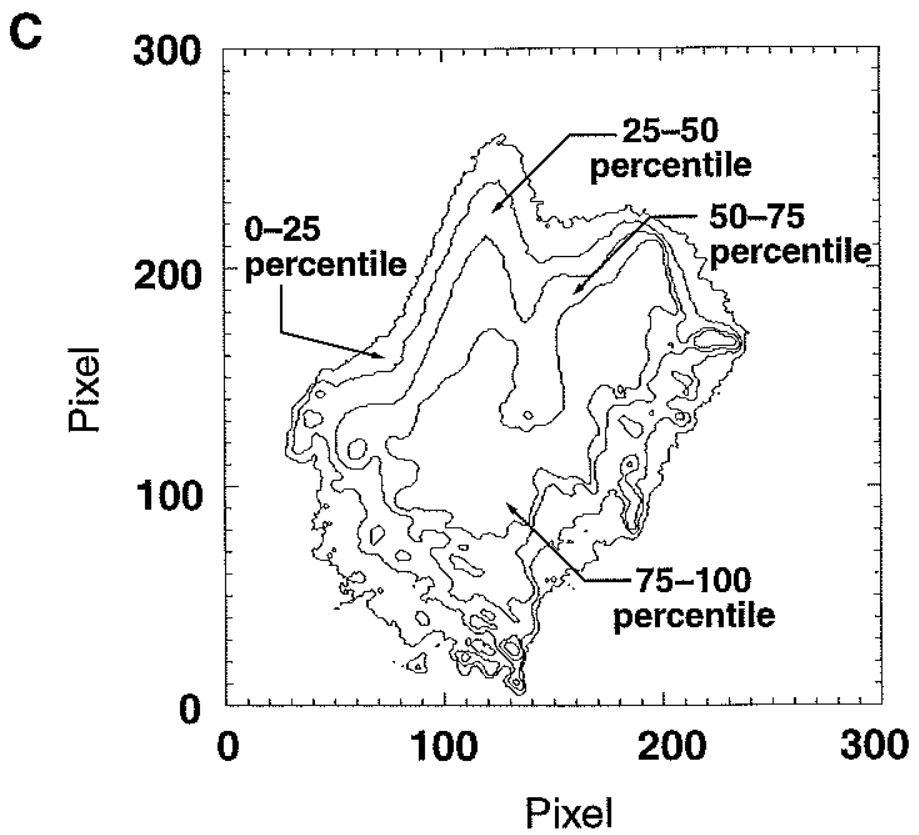

Figure 5.21 
Dive 3182: P Vent

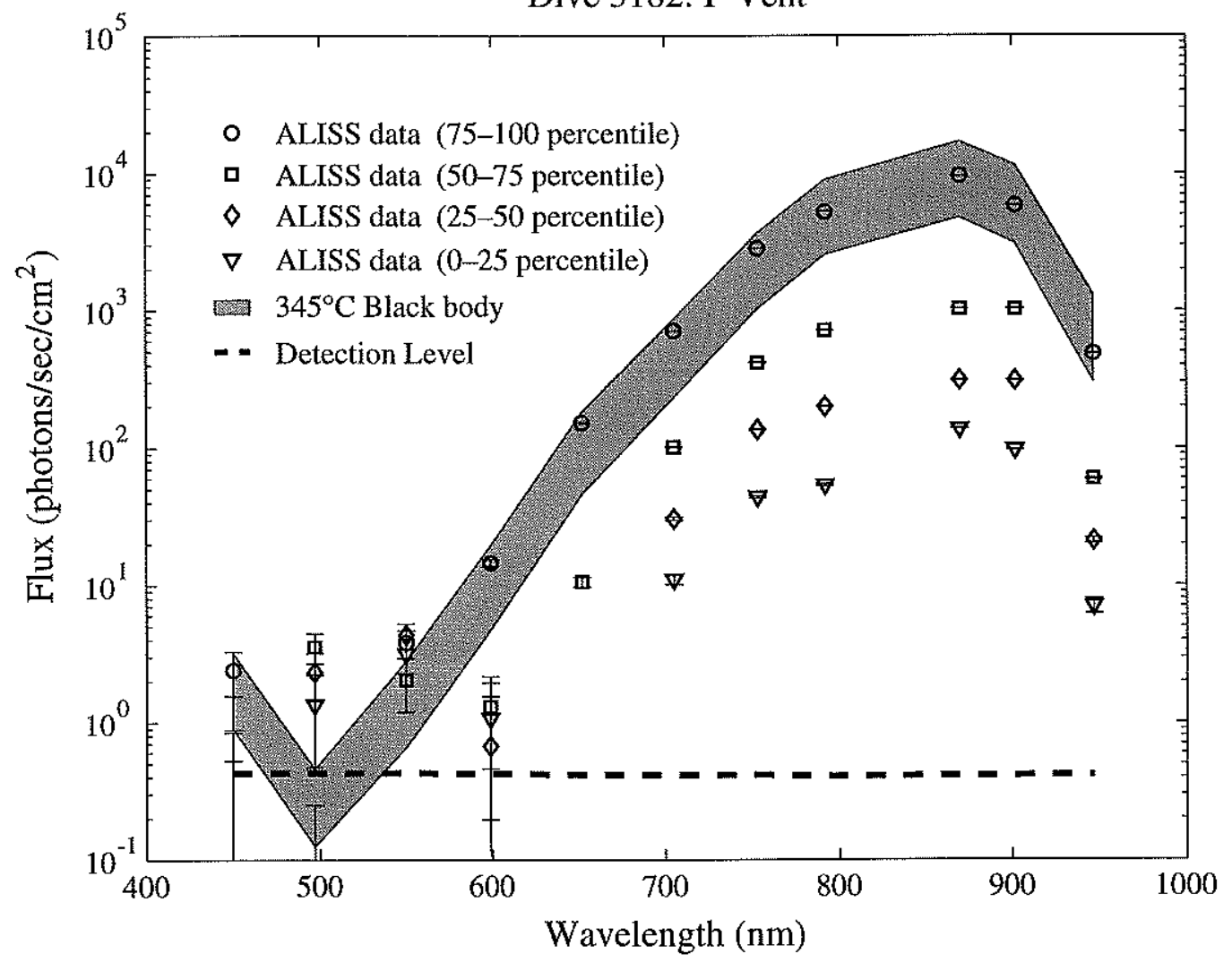

Figure 5.22 
Filter Array \#1

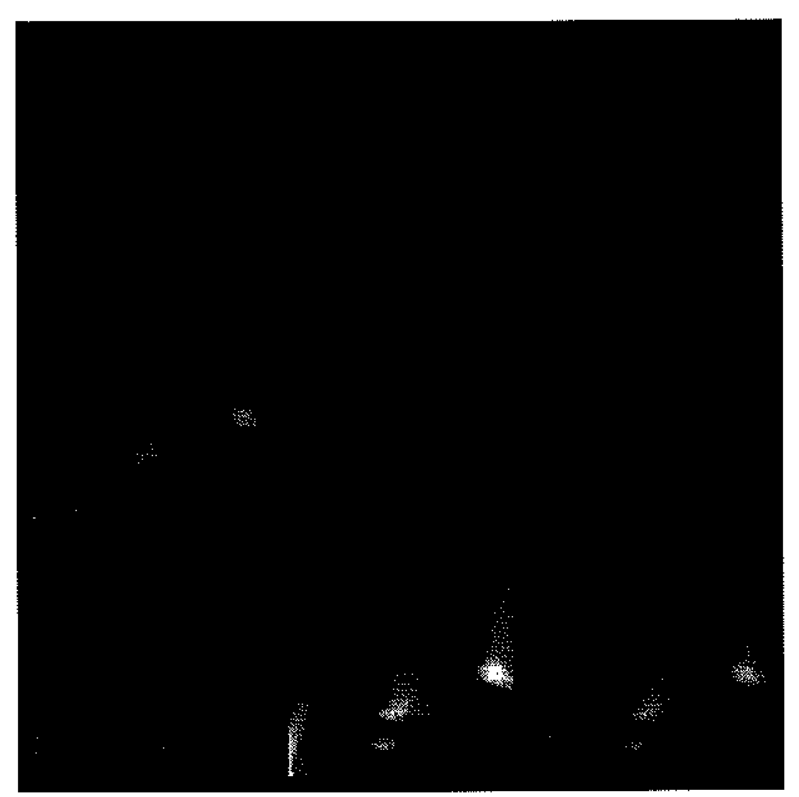

Filter Array \#2

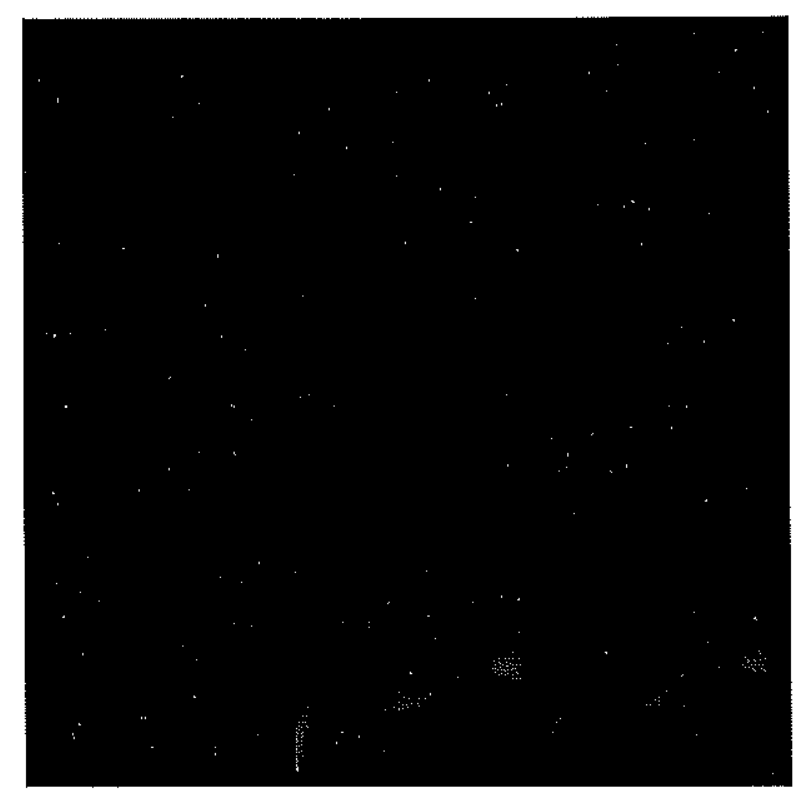

Figure 5.23 
A

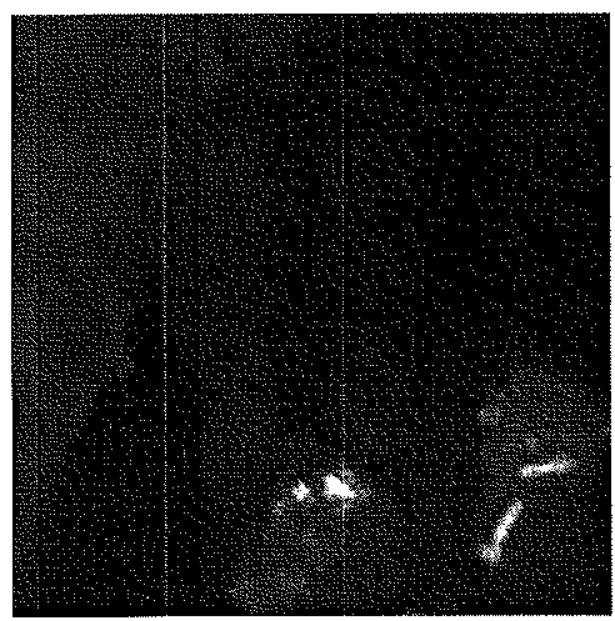

B

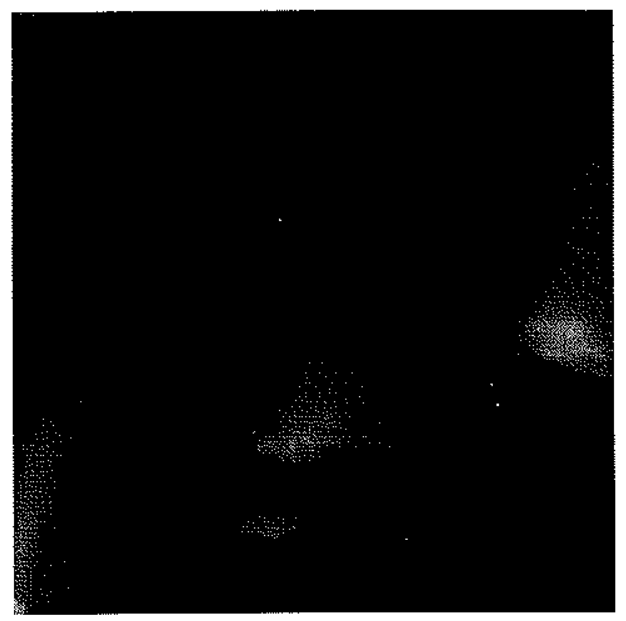

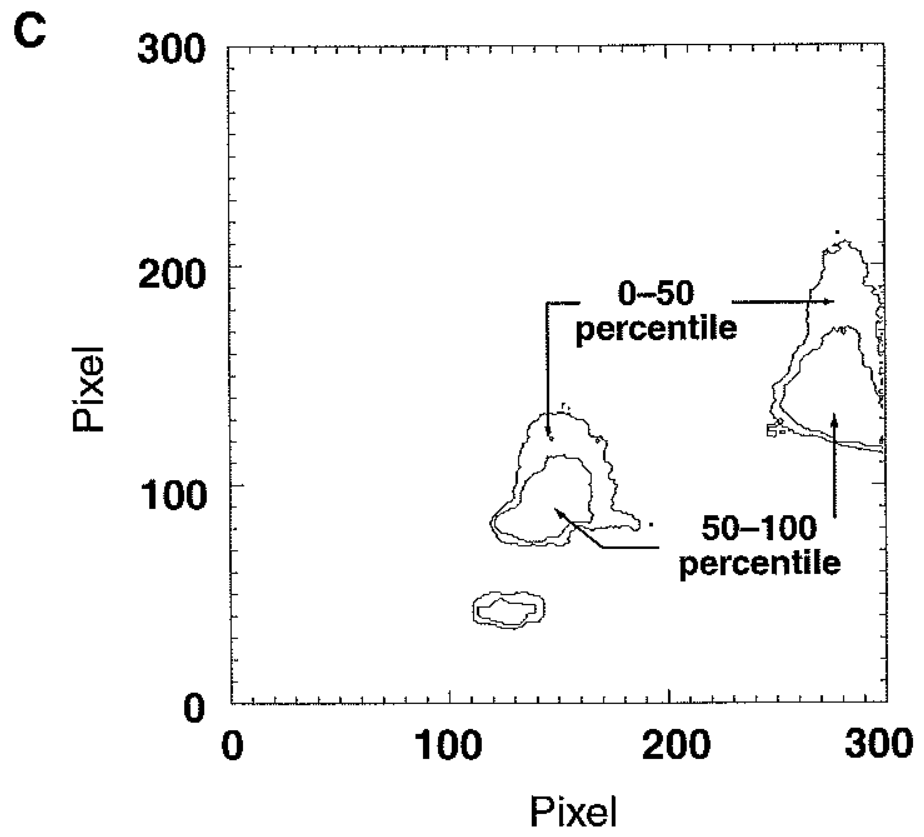

Figure 5.24 

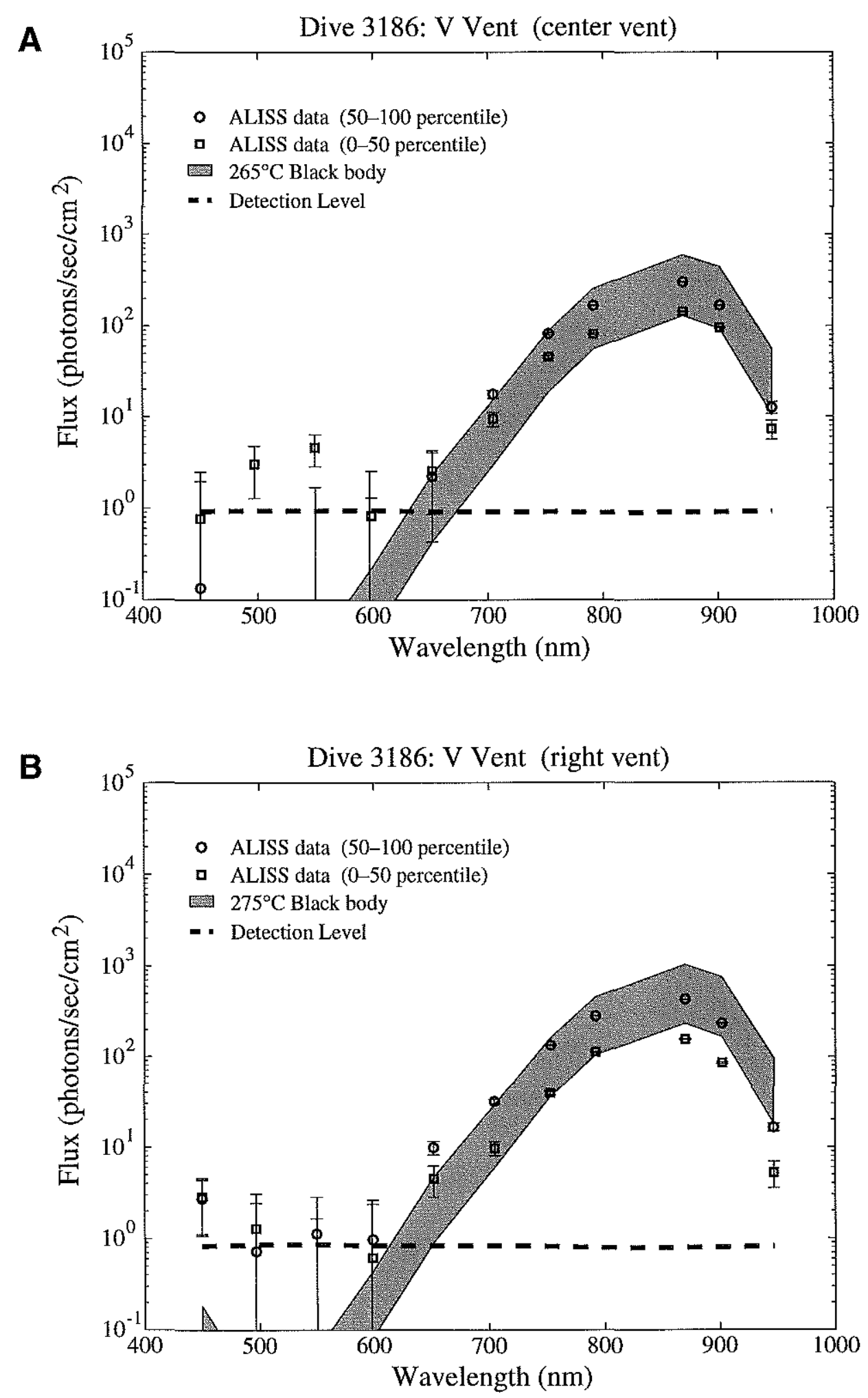

Figure 5.25 
Filter Array \#1

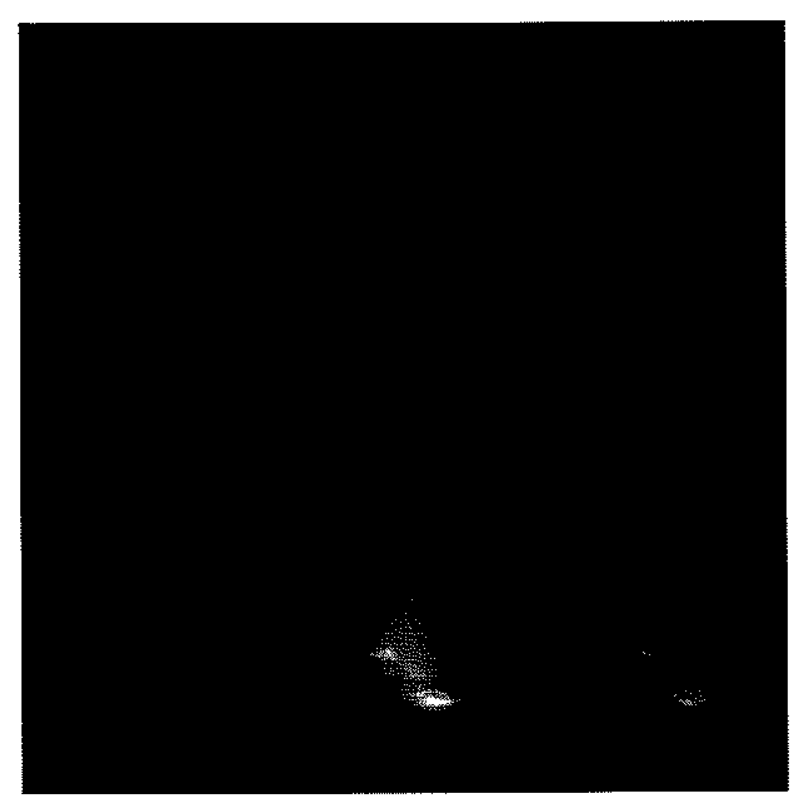

Filter Array \#2

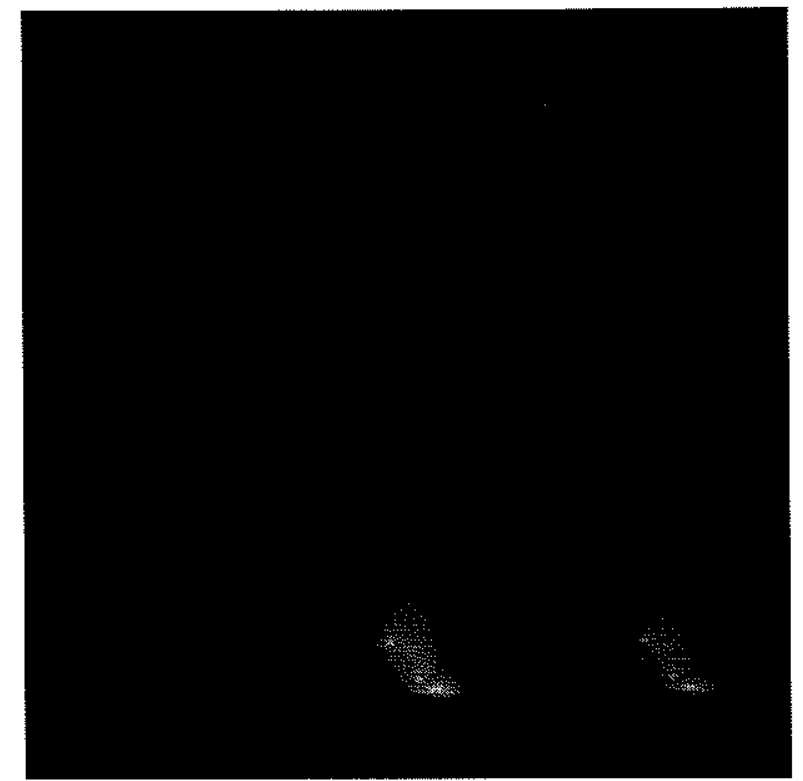

Figure 5.26 
A

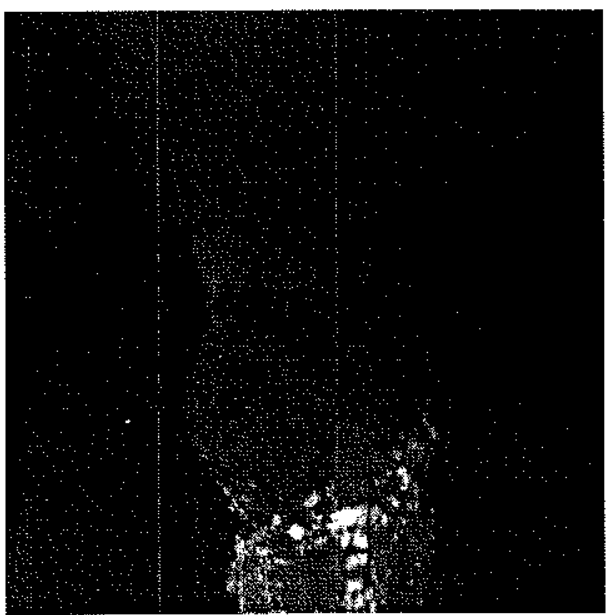

B

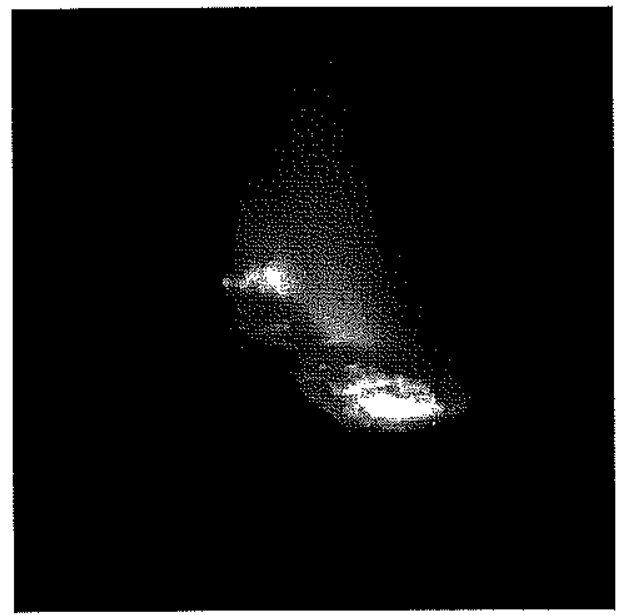

C

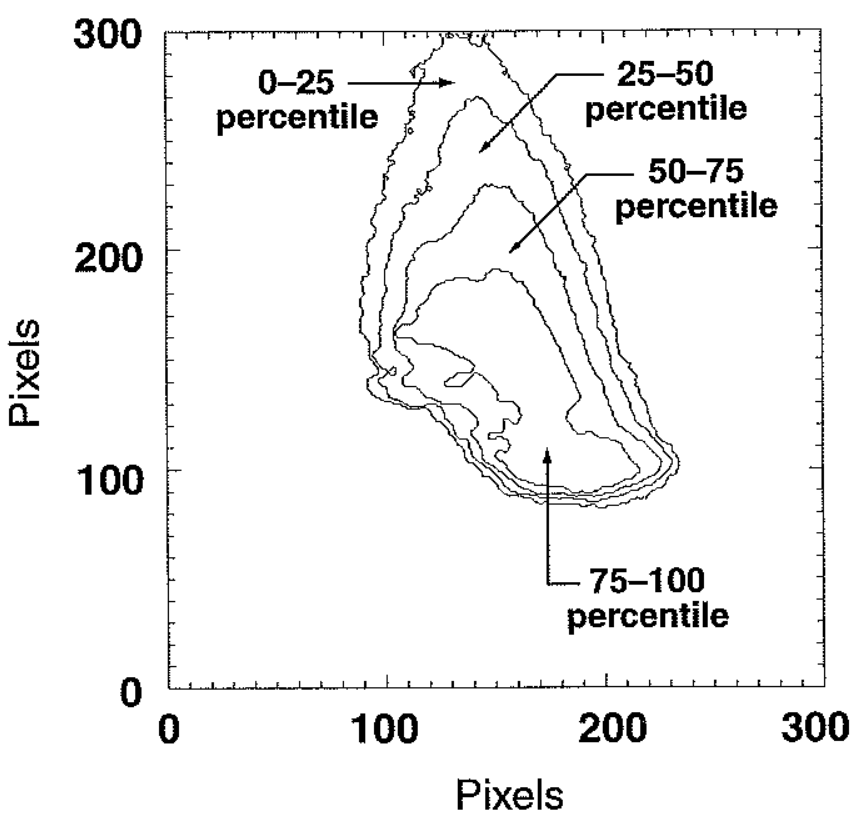

Figure 5.27 


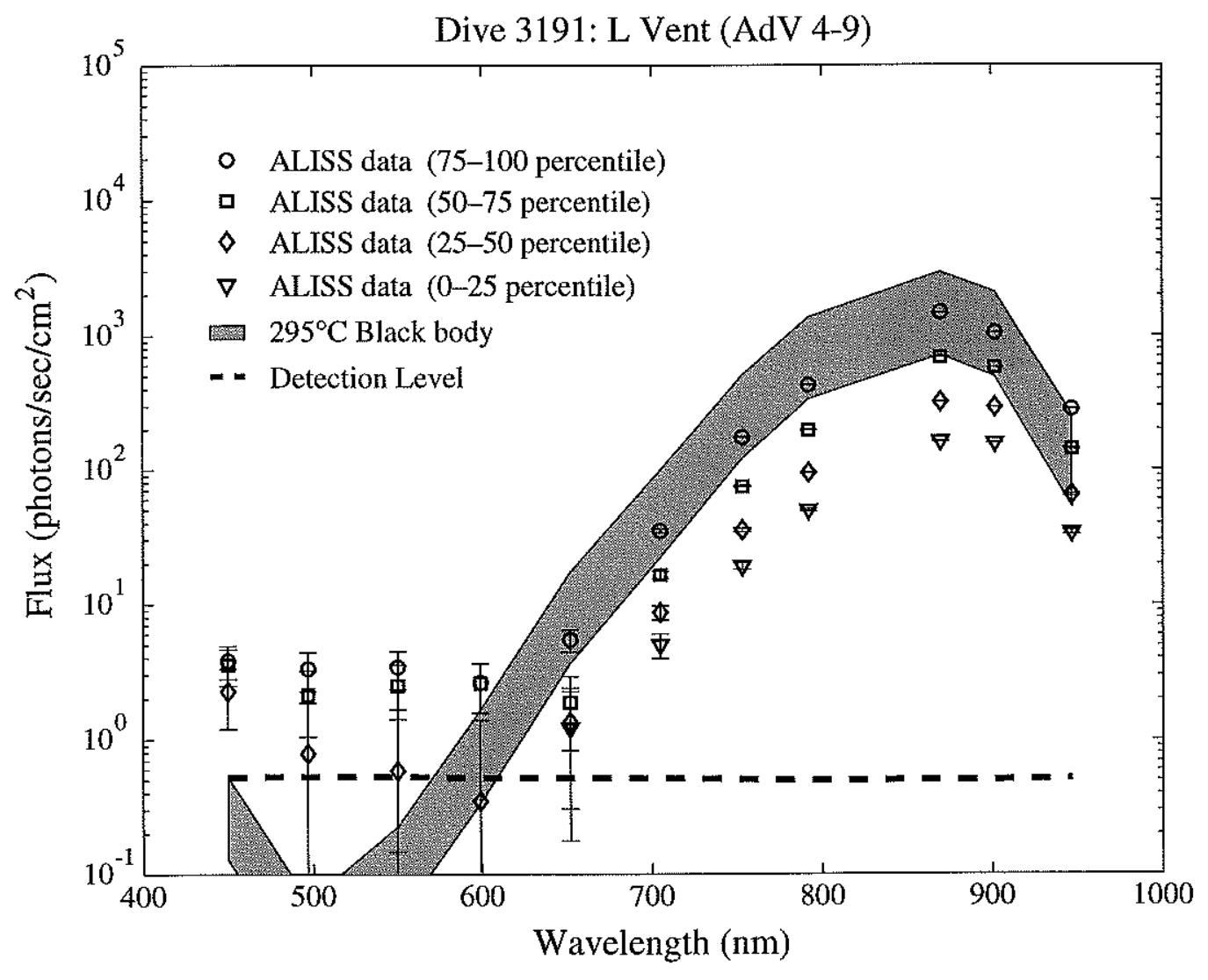

Figure 5.28 
A

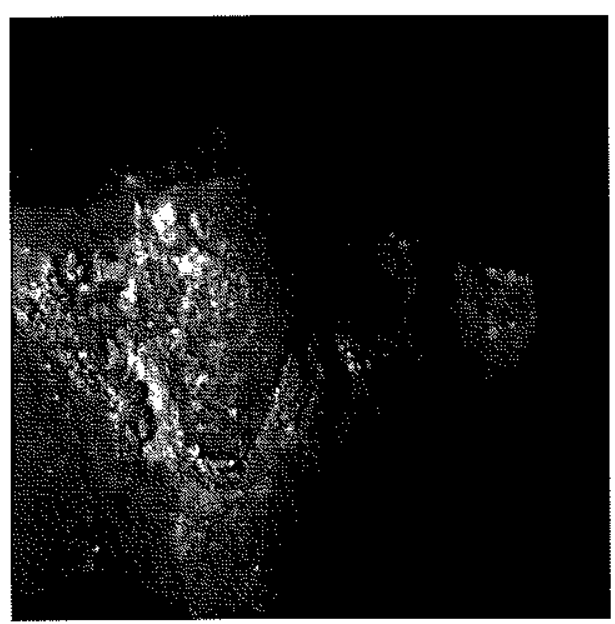

B

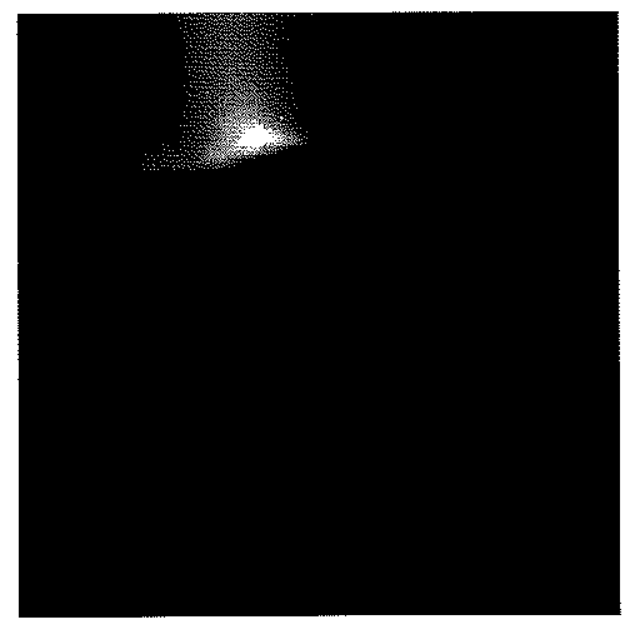

C

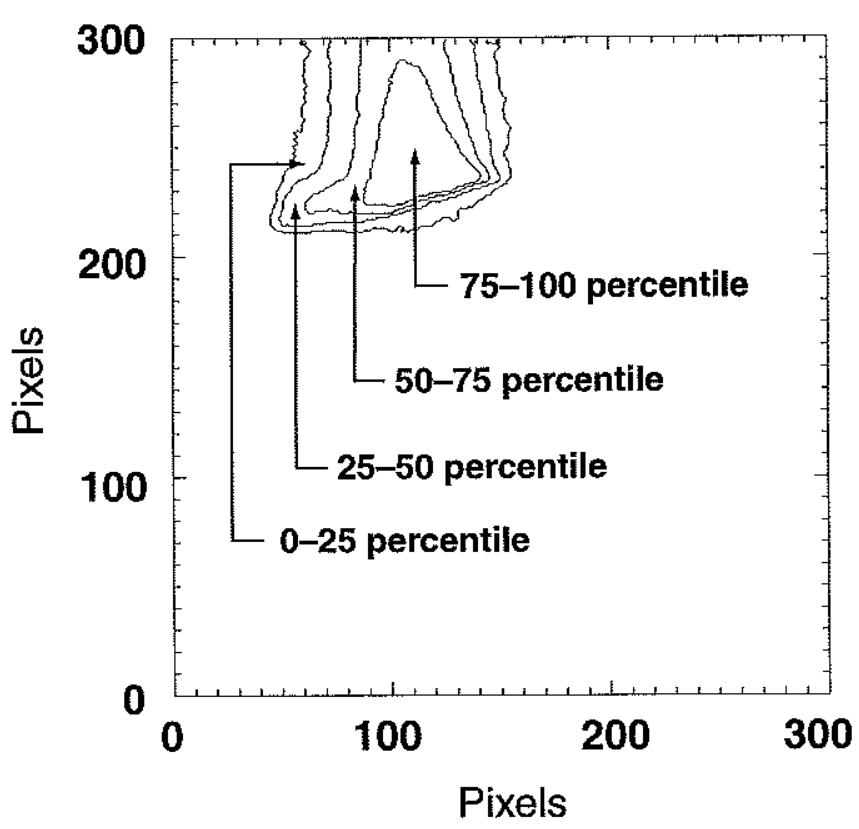

Figure 5.29 


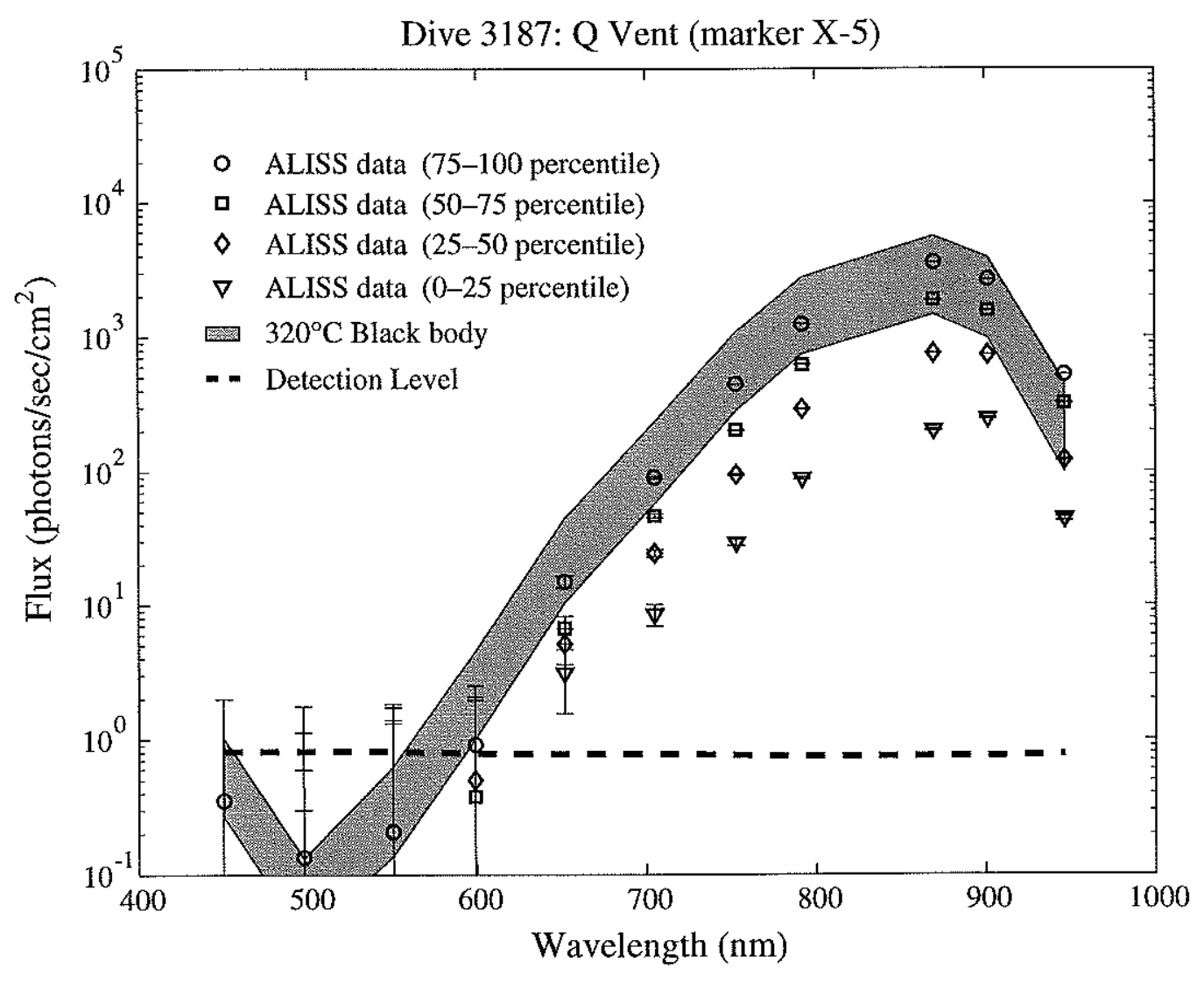

Figure 5.30 
A

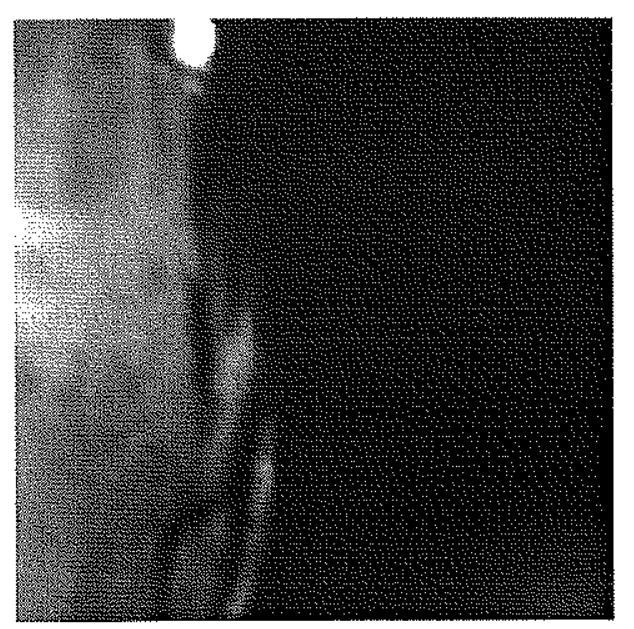

B

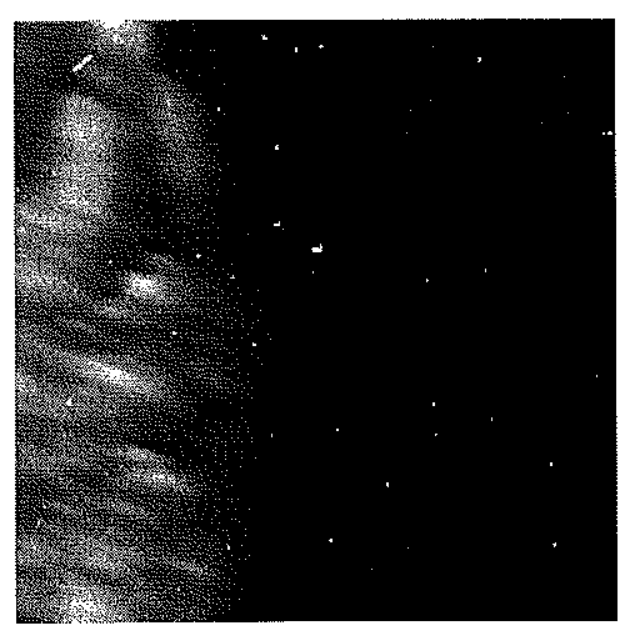

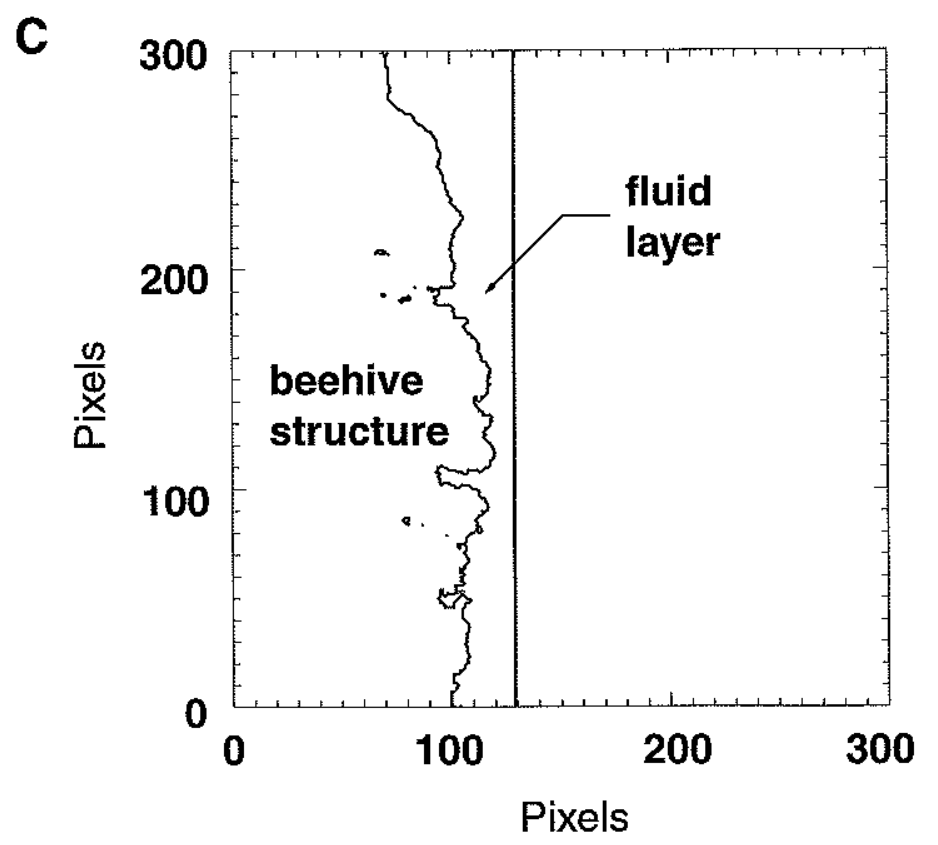

Figure 5.31 


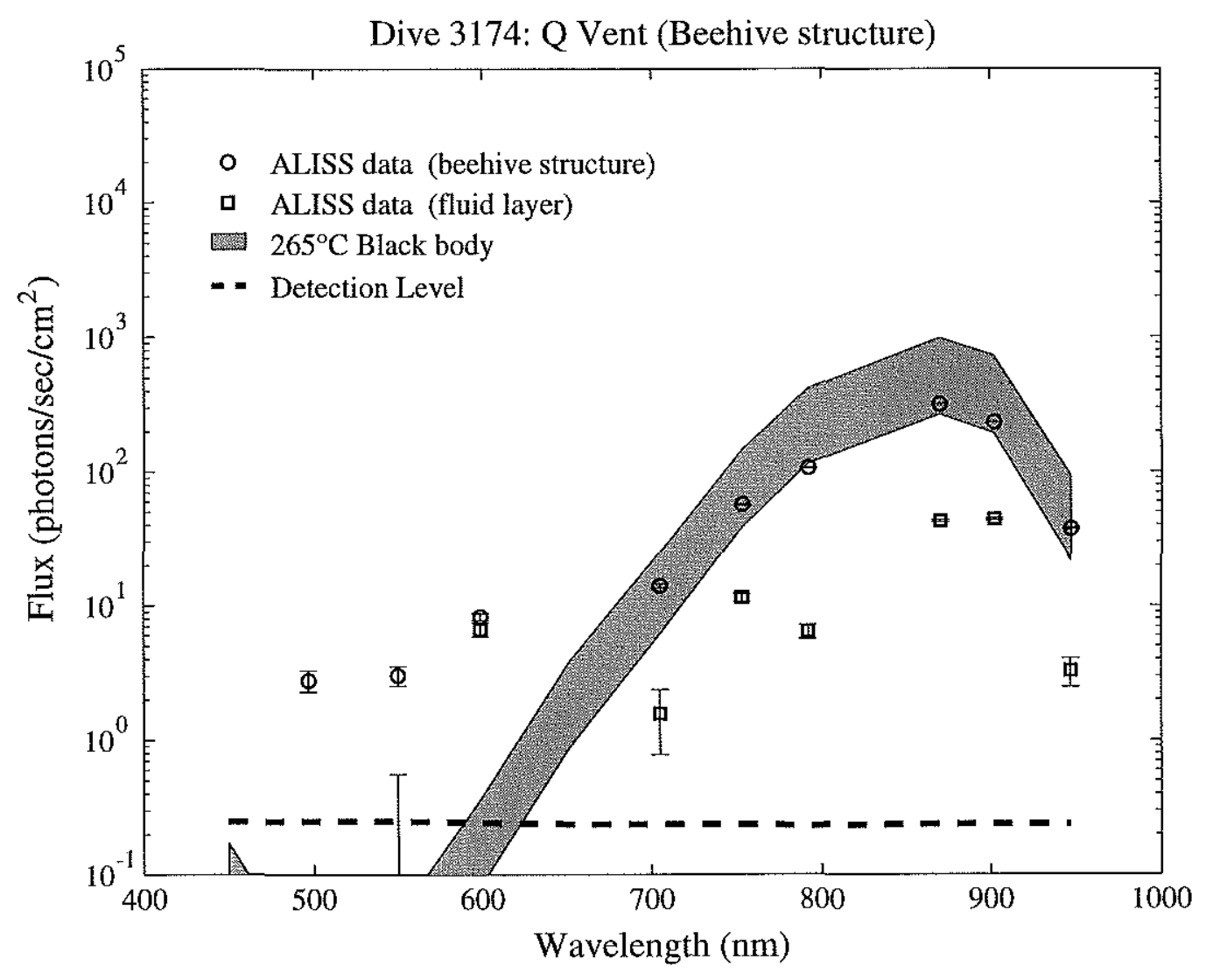

Figure 5.32 


\section{Chapter 6 - Conclusions, Implications, \& Future Work}

The phenomenon of light emission at hydrothermal vents is still a relatively new discovery (a little over 10 years old). By necessity, investigations of this phenomenon are exploratory in nature. As a result, our observations lead us to more questions than answers. Prior to this thesis research, three things were known about vents: (1) hightemperature, black smoker vents glow, (2) thermal radiation is one component of the emitted light, and (3) other non-thermal components may exist. Ambient light CCD images of a black smoker on the Juan de Fuca Ridge proved that high-temperature vents emit light [Smith and Delaney, 1989]. Subsequent calculations by Tyson [in LITE Workshop Participants, 1993] showed that the light imaged in the 750-850 nm band was consistent with thermal radiation. Two sets of data obtained in the 650-1050 nm region of the spectrum with a simple photometer further supported the existence of thermal radiation. However, the data also revealed higher than expected fluxes in the $650-750 \mathrm{~nm}$ band and temporal variations that suggested the presence of non-thermal light emission [Van Dover et al., 1996].

This thesis has expanded our knowledge of vent light in a number of ways. CCD technology was successfully adapted to obtain actual images of vent light in a number of wavelength bands providing information about the spatial distribution of vent light. A broader range of wavelengths were observed $(400-1000 \mathrm{~nm})$ simultaneously which helped both to better characterize visible light emission and to correlate temporal variability across bands. Finally, a variety of venting structures were imaged (black smokers, flange pools, and a beehive), allowing us to relate variations in light emission to variations in physical processes (e.g., turbulence, mixing, and precipitation). 


\subsection{Effectiveness of the ALISS Camera System}

The ALISS camera system was very effective in obtaining the spatial, spectral, and temporal data necessary to characterize light emission from deep-sea hydrothermal vents. The ability to image vent light in a number of wavelength bands simultaneously allowed detailed spectral analysis of the emission from various regions of the plume. The sensitivity of the camera also allowed short-exposure images to be obtained, which illustrated how rapidly light emission at vents can change.

The only significant problem with the ALISS camera system is the inability of filters to completely block light outside of their transmission band. Interference filters are designed to allow significant transmission $(\sim 60 \%)$ in a defined band. In the region outside of this band, transmission is reduced orders of magnitude. However, the light observed at hydrothermal vents varies by orders of magnitude between 400 and $1000 \mathrm{~nm}$. Thermal radiation from a $350^{\circ} \mathrm{C}$ black body increases 14 orders of magnitude from $400 \mathrm{~nm}$ to 1000 nm (Figure 2.1). Therefore, observations of a thermal source (such as a hydrothermal vent) with a $100 \mathrm{~nm}$ bandpass filter centered at $450 \mathrm{~nm}$ will include significant leakage at long wavelengths (>700 nm) (Figure 6.1).

As described in Section 3.1, ALISS used a set of filters 10,50 and $100 \mathrm{~nm}$ in bandwidth spanning the $400-1000 \mathrm{~nm}$ region. The objective of this arrangement was too provide $50 \mathrm{~nm}$ resolution across the region (i.e., data from the $50 \mathrm{~nm}$ filter and the difference between the date from the 100 and $50 \mathrm{~nm}$ filer). Unfortunately, given the significant leakage at long wavelengths, this could not in accomplished. Thus, the $50 \mathrm{~nm}$ filters provided superfluous data that did not greatly enhance spectral analysis. The $10 \mathrm{~nm}$ bandwidth filter centered at $589 \mathrm{~nm}$ was used to look for evidence of sonoluminescence, which exhibits strong emission at that wavelength. The bandwidth of this filter was too narrow to obtain a significant signal above the noise. 


\subsection{Implications for Light Sources at Hydrothermal Vents}

Light emission is indeed ubiquitous at hydrothermal vents. The dominant source of vent light is thermal radiation (Section 2.1) which is dependent solely on temperature and emissivity. Thermal radiation is observed at all types of vents and emits most strongly at wavelengths above $700 \mathrm{~nm}$. Due to their large size (on the order of $1 \mathrm{~m}^{2}$ ) and high emissivity $(\sim 0.9)$, flange pools emit a greater amount of thermal light than smaller black smoker vents. Due to their high flux and relative stability, flange pools provide favorable conditions for organisms which may use light to their advantage (discussed further in Section 6.3).

Some flanges (such as Dudley Flange) exhibit excess (non-thermal) light emission at $500 \mathrm{~nm}$ that is associated with solid material protruding through the hot flange pool into cold ambient seawater. It is possible that precipitation is actively occurring on these features, or that they induce local mixing. Analyses of the protruding material may help to determine whether processes such as crystalloluminescence or chemiluminescence are occurring. The ability to isolate one source mechanism at a flange will greatly help in assessing the importance of various mechanisms in more complicated black smokers.

In addition to thermal radiation, black smokers emit light in the visible region of the spectrum possibly due to a combination of non-thermal sources. At this time, we cannot confirm the contribution of the various sources because their emission spectra and intensity levels are not sufficiently well characterized. However, some preliminary conclusions can be drawn from the ALISS data. Cerenkov radiation, while present in the ocean, does not produce a high enough flux to be a significant component of vent light (Section 2.2). The amount of Cerenkov radiation produced by even high levels of ${ }^{222} \mathrm{Rn}$ is below the detection level of the ALISS camera. Sonoluminescence (Section 2.3) also does not appear to be a major contributor as the expected strong emission at $589 \mathrm{~nm}$ is not observed. Q Vent has 
the highest sodium concentration of all of the vents imaged (slightly higher than seawater), however, no significant emission around $589 \mathrm{~nm}$ is observed (Figures 5.30). The shallower depths of the Endeavour vents allow phase separation to occur at or just below the seafloor [Butterfield et al., 1994]. This suggests that bubble formation and hence sonoluminescence are more likely to occur at the Endeavour vents than at the $9^{\circ} \mathrm{N}$ vents. Vapor Bubble Luminescence (VBL) is a newly identified type of sonoluminescence. Its emission spectrum has not yet been characterized; however, it must be considered as a possible source of vent light.

Triboluminescence and crystalloluminescence (Section 2.4) as well as chemiluminescence (Section 2.5) remain possible sources of vent light. All three mechanisms require conditions (i.e., minerals and chemical reactions) present at hydrothermal vents, and they produce light in the visible region of the spectrum as is observed in the ALISS data. Most of the visible light emission appears to be generated in the plume a few centimeters away from the orifice where rapid mixing with ambient seawater leads to mineral precipitation and lower temperatures. In some cases, $450 \mathrm{~nm}$ light is observed in the orifice, which is consistent with crystallo- and/or triboluminescence from chalcopyrite - a mineral known to line chimney conduits (Figure 2.9). What cannot yet be explained is the presence of white light (uniform light emission across all bands) at the orifice observed at both Sully Vent (Figure 5.18) and L Vent (Figure 5.28). These two vents do not share similar physical properties and chemistries, and are not otherwise distinct from the rest of the vents imaged. The white light observed at these vents may be a combination of a number of sources emitting similar intensities in different regions of the spectrum. Thus, while this thesis research has improved our understanding of the spatial, spectral and temporal characteristics of vent light, much work remains to be done (discussed in Section 6.4). 


\subsection{Brological Consequences}

Hydrothermal vents are home to a unique biological community whose primary producers are chemosynthetic bacteria that extract energy from vent chemicals rather than sunlight [Jannasch, 1995]. These bacteria live symbiotically with more complex organisms such as tube worms or are grazed upon by heterotrophic organisms [Hessler and Kaharl, 1995; Van Dover, 2000]. Because of the depths at which these communities live (thousands of meters below the sea surface), their environments were thought to be void of light. Hence, it was not surprising to scientists to find that some shrimp (e.g., $R$. exoculata) lacked eyes [Williams and Rona, 1986]. However, we now know that nonsolar light does exist in the deep ocean, and that the supposedly blind shrimp have unique photoreceptors capable of detecting low levels of light [Van Dover et al., 1989; O'Neill et al., 1995]. The question must now be asked: How does vent light affect surrounding biological communities?

Are vent animals capable of seeing vent light, and if so, how do they use this information? As discussed in Section 1.2, the vent shrimp Rimicaris exoculata has developed a dorsal photoreceptor uniquely designed to detect low light levels [O'Neill et al., 1995]. Two other Mid-Atlantic vent shrimp (Chorocaris chacei and Chorocaris fortunata) were found to have similar photoreceptive organs [Van Dover, 2000, and references therein]. Calculations by Pelli and Chamberlain [1989] suggest that $R$. exoculata's photoreceptor is capable of detecting a $10 \mathrm{~cm}$ wide, $350^{\circ} \mathrm{C}$ vent from 2.3 meters away. However, their calculations are based on some assumptions that may not be correct. In calculating flux from a black body, the version of Planck's equation they use is not appropriate for flux by hot water radiating into water (i.e., they lack the $n^{2}$ term shown in Equation 2.2). They assume an ideal black body source (i.e., emissivity $=1.0$ ). However, the ALISS data suggest that black smoker vents have a much lower emissivity 
$(\sim 0.3)$. The attenuation through water was considered negligible. In fact, the intensity of $590 \mathrm{~nm}$ light (which they are considering) traveling through $2.3 \mathrm{~m}$ of seawater will be reduced by $20 \%$. Finally, the assumption of a $10 \mathrm{~cm}$ wide circular source implies a $78 \mathrm{~cm}^{2}$ emitting area. Image data collected with the ALISS camera suggest that the emitting area is closer to $10 \mathrm{~cm}^{2}$. Thus, it is not clear if $R$. exoculata can detect purely thermal radiation from a vent. Since black smokers often emit non-thermal light in the visible region of the spectrum, it may be possible for the shrimp to detect non-thermal black smoker light. However, as stated by Pelli and Chamberlain [1989], only behavioral evidence can prove that $R$. exoculata actually sees vent light.

Phototaxis is the movement of a motile organism in response to stimulation by light. This response can either be positive (toward the light) or negative (away from the light). The protozoan algal flagellate Euglena gracilis is known to exhibit phototactic behavior. Under low light conditions, Euglena is positively phototactic - it swims toward the light source. However, under high intensity conditions, it is negatively phototactic [Wolken, 1995]. If R. exoculata does see vent light, it may have one or both phototactic responses. Being able to see vents may lead the shrimp to food (i.e., the chemosynthetic bacteria prevalent at vents), or away from vent fluid hot enough to cook them [Van Dover, 2000]. Unfortunately, behavioral observations have not been made because the bright lights of submersibles tend to blind the shrimp.

A more challenging (and controversial) question is: Does photosynthesis occur at deep-sea hydrothermal vents? A number of phototrophic organisms are adapted to live in low-light conditions. These include organisms living at the bottom of the pelagic photic zone, in shallow benthic environments, and beneath polar ice. Bacteria appear to adapt well to low light conditions. Five strains of a photosynthetic, brown, sulfur bacterium (Chlorobium phaeobacteriodes) were isolated from a depth of $80 \mathrm{~m}$ in the Black Sea. All 
strains were found to be extremely low-light adapted, thriving at light intensities $<1$ $\mu$ Einst $/ \mathrm{m}^{2} / \mathrm{sec}\left(6 \times 10^{13}\right.$ photons $\left./ \mathrm{cm}^{2} / \mathrm{sec}\right)$. In fact, the amount of light reaching $80 \mathrm{~m}$ depth was calculated to be $\sim 0.01 \mu$ Einst $/ \mathrm{m}^{2} / \mathrm{sec}\left(6 \times 10^{11}\right.$ photons $\left./ \mathrm{cm}^{2} / \mathrm{sec}\right)$ [Overmann et al., 1992]. Species of green bacteria (e.g., genera Pelodictyon and Ancalochloris) and purple bacteria are also able to survive at low light levels $\left(\sim 1-2 \mu\right.$ Einst $\left./ \mathrm{m}^{2} / \mathrm{sec}\right)$ and low sulfide concentrations [Pfennig, 1978].

For an organism to be able to photosynthesize using vent light, it must contain pigments that absorb at long wavelengths, and it would have to live close to the vent fluid where attenuation effects are minimal. Bacteria contain bacteriochlorophylls which absorb light at both short wavelengths and long (red and infrared) wavelengths [Pfennig, 1978]. Figure 6.2 shows the absorption by bacteriochlorophylls and other photosynthetic pigments. Thus, the most probable candidate for a hydrothermal phototroph is a purple sulfur bacterium. Deep-sea vents can provide both the sulfide and light necessary for such bacteria to thrive. Purple bacteria absorb in the $880-1040 \mathrm{~nm}$ range due to the presence of bacteriochlorophylls. Given the shape of the black body radiation curve and the effects of attenuation, a significant amount of light is emitted in this wavelength band. Figure 6.3 shows the flux from a $330^{\circ} \mathrm{C}$ flange pool (emissivity 0.9 ) radiating into a half-space. The dotted line indicates the $0.01 \mu$ Einst $/ \mathrm{m}^{2} / \mathrm{sec}$ level noted in Overmann et al. [1992]. In the 880-1040 nm region where bacteriochlorophylls absorb, the flux from a flange pool is the same order of magnitude as that reaching $80 \mathrm{~m}$ depths in the Black Sea. Thus, it certainly seems possible for photosynthesis to occur at vents. However, the attenuation of light in seawater is strong at those wavelengths $\left(\sim 0.2 \mathrm{~cm}^{-1}\right.$ at $\left.950 \mathrm{~nm}\right)$. At a distance of $3.5 \mathrm{~cm}$ from the flange, the intensity drops by half. At $11.5 \mathrm{~cm}$ away the intensity is reduced to one tenth of that at the flange pool. If phototrophic organisms exist at vents, they must be specially adapted to low levels of light or live very close to the hydrothermal fluid. 
Additionally, due to the ephemeral nature of vents, photosynthesis is most likely to be facultative rather than obligate.

At present, no known phototrophs have been identified at deep-sea vents. Yurkov and Beatty [1998] report the isolation of bacteria with bacteriochlorophyll absorption maxima at $867 \mathrm{~nm}$ from two water samples collected tens of meters above a vent field on the Juan de Fuca Ridge. However, the existence of surface-water contaminants in the sample remains a possibility [C. Van Dover, per. comm.].

\subsection{Future WORK}

As with any research, there is always more to be done. This is especially true of newly identified phenomena such as vent light. In this section, I outline some of the important next steps to be taken to increase our understanding of light emission at hydrothermal vents.

\subsection{Ambient IMAGING OF VENTS AT TAG HydROTHERMAL FieLd}

As noted in the Introduction (Chapter 1), the discovery of vent light was prompted by the unique photoreceptor found on the vent shrimp $R$. exoculata. This species of shrimp is found only at vents on the Mid-Atlantic Ridge (MAR) and their eyes absorb maximally at $500 \mathrm{~nm}$ [Van Dover et al., 1989]. Only Pacific vents were imaged with the ALISS camera system, and none showed consistent, significant light emission at $500 \mathrm{~nm}$. So, what are the shrimp looking at? It is possible that visible light emission of Mid-Atlantic vents differs from that of Pacific vents. The East Pacific vents imaged by ALISS (on the East Pacific Rise and the Juan de Fuca Ridge) lie at depths of 2200-2500 m [Crane et al., 1985; Haymon et al., 1993], while the TAG Hydrothermal Field on the MAR lies at a depth of $\sim 3620-3700 \mathrm{~m}$ [Rona et al., 1986]. This is about a 120 bar difference in 
pressure. The 30 bar difference between the Juan de Fuca and EPR vents would not be expected to have a major effect on light emission, and indeed the emission from those vents is quite similar. However, the 120 bar difference may, in fact, have a significant effect on the light observed on the MAR. As noted in Section 2.3, pressure can have an effect on sonoluminescent photon flux. However, at a depth of $3700 \mathrm{~m}$ bubble generation and consequently sonoluminescence may not be possible. Other light emission mechanisms may also be affected or hindered by the higher pressures. Just as many of the possible sources are not well characterized, the affect of extreme pressure on those sources is not known. Thus, it is likely that the spectrum of vent light is different in the Atlantic compared to the Pacific, but the significance of that difference cannot be quantified a priori.

It should also be noted that the biological communities at Atlantic and Pacific vents are different. The East Pacific vents are dominated by tube worms, clams, and mussels, while the Mid-Atlantic vents are dominated by shrimp [Hessler and Kaharl, 1995]. The confinement of $R$. exoculata (and other shrimp with similar photoreceptors) to the Atlantic may be a result of limitations in larval dispersion, or it may be a result of favorable (i.e., visible) light conditions at Atlantic vents that are not present at Pacific vents. Thus, obtaining ambient light measurements at Mid-Atlantic vents are important to better understand the shrimp's environment, and to characterize vent light in the Atlantic and the effect of higher pressures.

\subsubsection{Characterization Vent FluId/SEAWATER PROPERTIES}

As discussed in Chapter 4, the attenuation of seawater in vent environments and the emissivity of vent fluids are important physical properties in interpreting ambient light data. The analyses presented in this thesis used long-wavelength light emission and measured vent temperatures to estimate values of emissivity. However, it is likely that emissivity 
varies with wavelength, and possibly from vent to vent. The experiment discussed in Section 4.3 will help to characterize vent fluid emissivity allowing us to more accurately determine the contribution of thermal radiation to vent light.

Because ambient light measurements are made at some distance from the vent, the attenuation of light in seawater is an important factor in determining the actual flux at the vent. Previous attempts to measure attenuation in situ yielded questionable results (Section 4.2.1). The attenuation values from the literature, used to calculate how much predicted thermal radiation reached the ALISS camera through $50 \mathrm{~cm}$ of water, provided a good fit with the ALISS data at long wavelengths. However, attenuation in the visible region was

underestimated because scattering was neglected. Attenuation measurements at the seafloor cannot easily be made at the same resolution as laboratory measurements (e.g., every 10 $\mathrm{nm}$ ). This degree of resolution, which corresponds to the resolution of the ALISS calibration measurements, is necessary for detailed calculations. Therefore, future attempts to measure attenuation in situ should be made, both to check of the literature values at long wavelengths, and to characterize the effect of scattering in the visible region.

\subsubsection{Characterization NON-THERMAl MECHANISMS}

A number of possible sources of vent light were discussed in Chapter 2. While some of these sources (e.g., thermal radiation and Cerenkov radiation) are well characterized, many are not. Without detailed information regarding both the spectra and the intensity of emitted light, it is difficult to determine the importance of the various sources. We have a basic understanding of the chemical reactions and minerals present at deep-sea hydrothermal vents, and know that a number of them emit light in the visible region of the spectrum. For example, chemiluminescence from sulfide oxidation is a possible source of vent light [Tapley et al., 1999]; however, its spectra and emission levels 
are not quantified. If these properties were known, not only could the contribution of chemiluminescence to vent light be ascertained, but ambient light images of vents in the proper wavelength band also could be used to show the distribution of sulfide oxidation above the vent orifice.

An increased understanding of various sources of light emission in the deep-sea would allow new types of instruments to be developed that use light as a diagnostic tool. The deep ocean, with its natural filtering of extraneous light, is tough but excellent place in which to make light measurements. It may be possible in the future to conduct in situ analyses of physical properties using fluorescence, and photoluminescence - methods currently used in laboratories. 


\section{Figure Captions}

Figure 6.1 Flux from a $350^{\circ} \mathrm{C}$ black body source with $10^{3}$ photons $/ \mathrm{cm}^{2} / \mathrm{sec} / \mathrm{str}$ of white light added (dash-dotted line). The dashed line indicates the flux after attenuation by $50 \mathrm{~cm}$ of water (see Figure 4.3 ). The solid line indicates the flux that passes through the ALISS optics and a $450 \mathrm{~nm}$ filter (see Figure 3.7). The leakage at long wavelengths is the same order of magnitude as at the flux through the intended pass band.

Figure 6.2 Absorption of radiation by photosynthetic pigments. Bacteriochlorophylls absorb at both short and long wavelengths. [From Valiela, 1995]

Figure 6.3 Predicted flux from a $330^{\circ} \mathrm{C}$ flange pool with an emissivity of 0.9 radiating into a half-space (i.e., solid angle $=2 \pi$ ). Dashed lines indicate region of maximum absorption by bacteriochlorophylls $(880-1040 \mathrm{~nm})$. The dotted line indicates the $0.01 \mu$ Einst $/ \mathrm{m}^{2} / \mathrm{sec}$ level at which photosynthetic, brown, sulfur bacteria in the Black sea live [Overmann et al., 1992]. The shaded area indicates the visible region of the spectrum. 


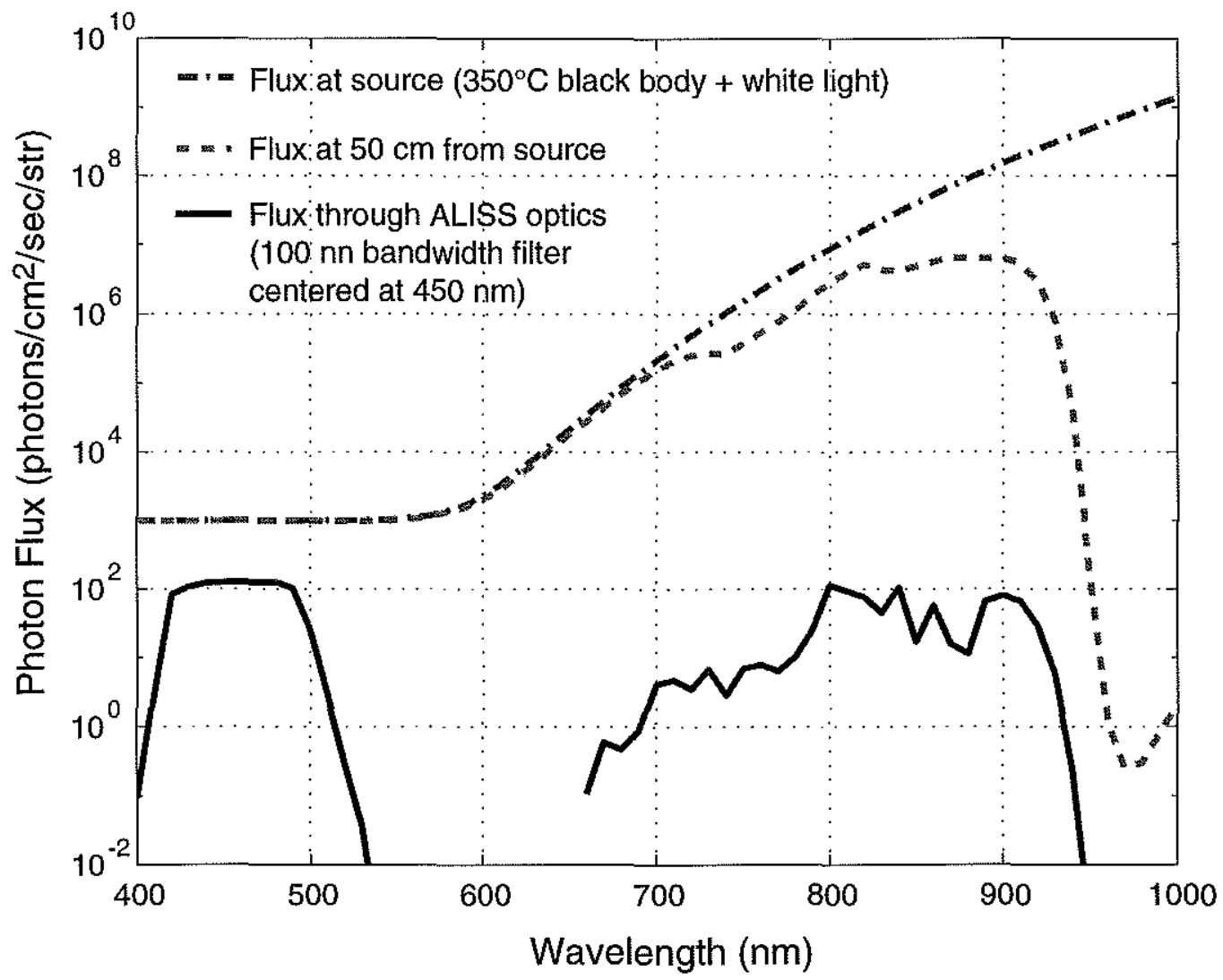

Figure 6.1 


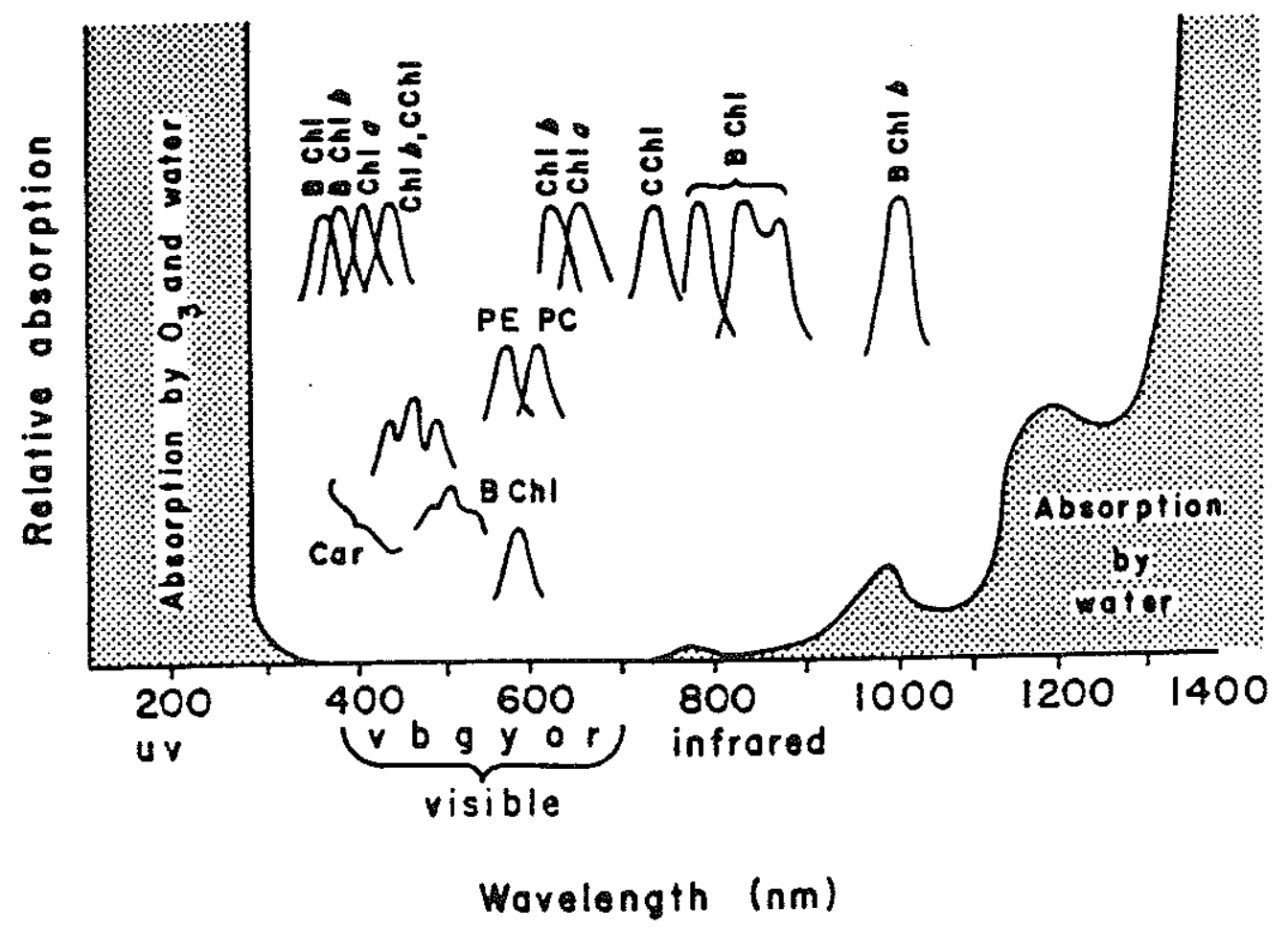

Figure 6.2 


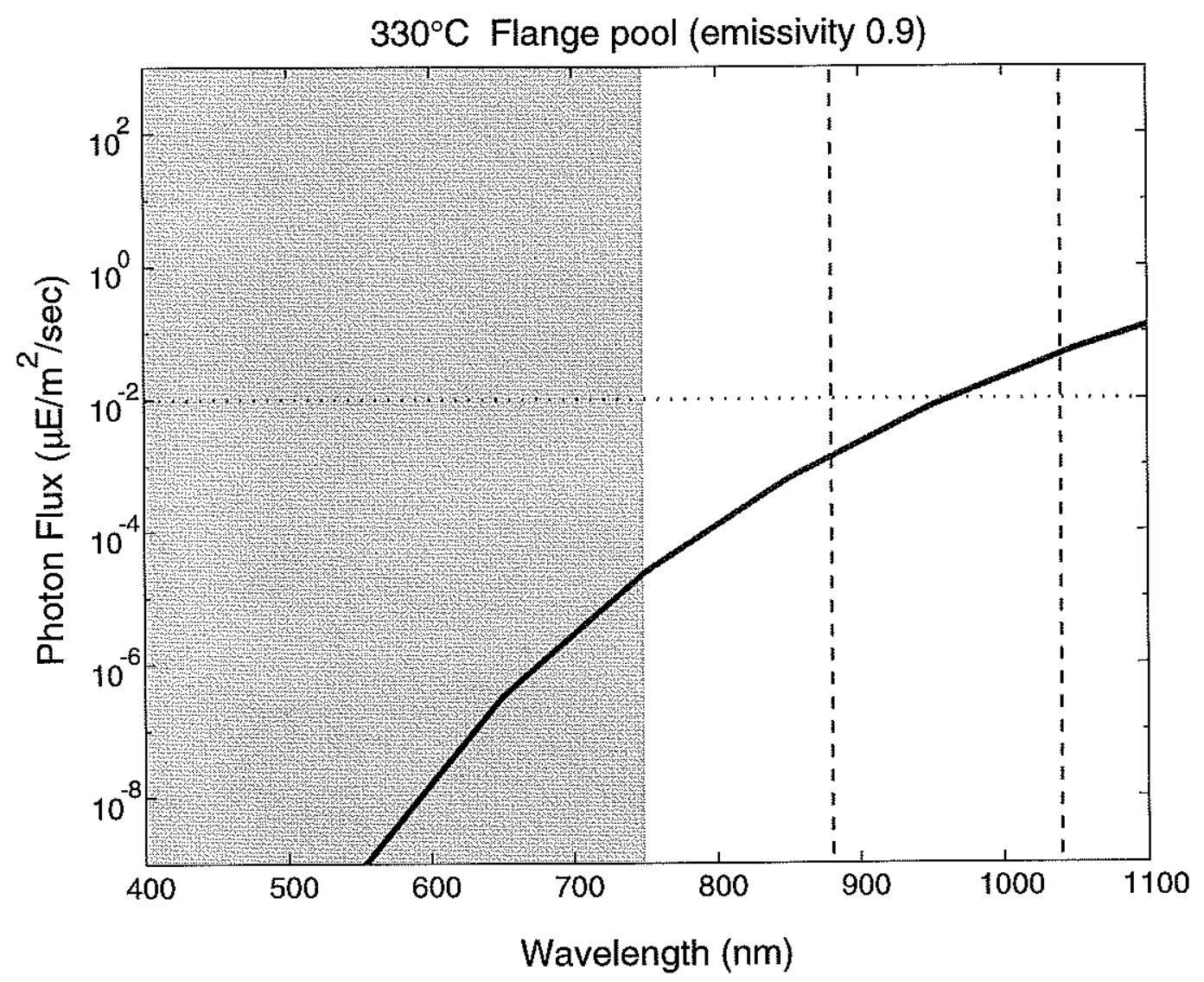

Figure 6.3 


\section{REFERENCES}

Alt, J.C., Subseafloor processes in mid-ocean ridge hydrothermal systems, in Seafloor Hydrothermal Systems: Physical, Chemical, Biological, and Geological Interactions, edited by S.E. Humphris, R.A. Zierenberg, L.S. Mullineaux, and R.E. Thomson, AGU, Washington, DC, 1995.

Anbar, M., Cavitation during impact of liquid water on water: geochemical implications, Science, 161, 1343-1344, 1968.

Barber, B.P., and S.J. Putterman, Observation of synchronous picosecond sonoluminescence, Nature, 1991, 318-320, 1991.

Baron, S.R., Hydrothermal vent precipitates: A study of temporal and geographic variations in black smoker particles and chimneys from 9-10 degrees N, East Pacific Rise, Doctoral thesis, University of California, Santa Barbara, Santa Barbara, 1998.

Bayly, J.G., V.B. Kartha, and W.H. Stevens, The absorption spectra of liquid phase $\mathrm{H}_{2} \mathrm{O}, \mathrm{HDO}$ and $\mathrm{D}_{2} \mathrm{O}$ from $0.7 \mu \mathrm{m}$ to $10 \mu \mathrm{m}$, Infrared Physics, 3, 211-223, 1963.

Becker, L., J.L. Bada, K. Kemper, and K.S. Suslick, The sonoluminescence spectrum of seawater, Marine Chemistry, 40, 315-320, 1992.

Beese, W., and J.I. Zink, The intensity of triboluminescence, Journal of Luminescence, 29, 119-122, 1984.

Behr, B., CCD Quantum Efficiency Test (QETest) System v1.0 User's Manual, California Institute of Technology, Palomar CCD Laboratory, 1996.

Belcher, E.H., The luminescence of irradiated transparent media and the Cerenkov effect: I. The luminescence of aqueous solutions of radioactive isotopes, Proc. Roy. Soc., A216, 90-102, 1953.

Bischoff, J.L., and K.S. Pitzer, Phase relations and adiabats in boiling seafloor geothermal systems, Earth Planet. Sci. Lett., 75, 327-338, 1985.

Bischoff, J.L., and W.E. Seyfried, Hydrothermal chemistry of seawater from $25^{\circ}$ to $350^{\circ}$ C, Am. J. Sci., 278 (6), 838-860, 1978.

Boyle, W.S., and G.E. Smith, Charge coupled semiconductor devices, Bell System Technical Journal, 49, 587-592, 1970.

Bradner, H., M. Bartlett, G. Blackington, J. Clem, K. D., J. Learned, A. Lewitus, S. Matsuno, D. O'Connor, W. Peatman, M. Reichle, C. Roos, J. Waters, M. Webster, and M. Yarbrough, Bioluminescence profile in the deep Pacific Ocean, Deep Sea Research, 34, 1831-1840, 1987.

Buil, C., CCD Astronomy: Construction and Use of an Astronomical CCD Camera, 321 pp., Willmann-Bell, inc., Richmond, VA, 1991.

Butterfield, D.A., R.E. McDuff, M.J. Mottl, M.D. Lilley, J.E. Lupton, and G.J. Massoth, Gradients in the composition of hydrothermal fluids from the Endeavour segment vent field: phase separation and brine loss, J. Geophys. Res., 99 (B5), 9561-9583, 1994. 
Campbell, A.C., T.S. Bowers, C.I. Measures, K.K. Falkner, M. Khadem, and J.M. Edmond, A time series of vent fluid compositions from $21^{\circ} \mathrm{N}$, East Pacific Rise $(1979,1981,1985)$, and the Guaymas Basin, Gulf of California (1982, 1985)., $J$. Geophys. Res., 93 (B5), 4537-4559, 1988.

Chen, K.Y., A.M. ASCE, and J.C. Morris, Oxidation of sulfide by $\mathrm{O}_{2}$ : Catalysis and inhibition, Journal of the Sanitary Engineerin Division, ASCE, SA1, 215-227, 1972.

Collins, G.B., and V.G. Reiling, Cerenkov radiation, Phys. Rev., 54, 499-503, 1938.

Collins, J.R., Change in the infrared absorption spectrum of water with temperature, Physics Reviews, 25, 771-779, 1925.

Crane, K., F. Aikman, R. Embley, S. Hammond, A. Malahoff, and J. Lupton, The distribution of geothermal fields on the Juan de Fuca Ridge, Journal of Geophysical Research, 90 (B1), 727-744, 1985.

Crum, L.A., Sonoluminscence, sonochemistry, and sonophysics, J. Acoust. Soc. Am., 95 (1), 559-652, 1994.

Crum, L.A., and G.T. Reynolds, Sonoluminescence produced by "stable" cavitation, Journal of the Acoustical Society of America, 78, 137-139, 1985.

Curcio, J.A., and C.C. Petty, The near infrared absorption spectrum of liquid water, Journal of the Optical Society of America, 41, 302-304, 1951.

Dan, M., J.D.N. Cheeke, and L. Kondic, Ambient Pressure Effects on single-bubble sonoluminescence, Phys. Rev. Lett., 83 (9), 1870-1873, 1999.

Delaney, J.R., V. Robigou, and McDuff, Geology of a vigorous hydrothermal system on the Endeavour Segment, Juan de Fuca Ridge, Journal of Geophyical Research, 97 (B13), 19,663-19,682, 1992.

Dera, J., Marine Physics, 516 pp., Elsevier, Amsterdam, 1992.

Didenko, Y.T., and S.P. Pugach, Specta of water sonoluminescence, J. Phys. Chem., 98, 9742-9749, 1994.

Edmond, J.M., C. Measures, R.E. McDuff, L.H. Chan, R. Collier, B. Grant, L.I. Gordon, and J.B. Corliss, Ridge crest hydrothermal activity and the balances of the major and minor elements in the ocean: The Galapagos data, Earth Planet. Sci. Lett., 46, 1-18, 1979.

Fegley, B., Properties and composition of the terrestrial oceans and of the atmospheres of the earth and other planets, in Global Earth Physics: A Handbook of Physical Constants, edited by T.J. Ahrens, AGU, Washington, DC, 1995.

Finkelnburg, W., Conditions for blackbody radiation of gases, J. Opt. Soc. Am., 39 (2), 185-186, 1949.

Flint, E.B., and K.S. Suslick, The temperature of cavitation, Science, 253, 1397-1399, 1991a.

Flint, E.B., and K.S. Suslick, Sonoluminescence from Alkali-Metal Salt Solutions, $J$. Phys. Chem., 95, 1484-1488, $1991 \mathrm{~b}$. 
Fornari, D.J., and R.W. Embley, Tectonic and volcanic controls on hydrothermal processes at the mid-ocean ridge: an overview based on near-bottom and submersible studies, in Seafloor Hydrothermal Systems: Physical, Chemical, Biological, and Geological Interactions, edited by S.E. Humphris, R.A. Zierenberg, L.S. Mullineaux, and R.E. Thomson, AGU, Washington, D. C., 1995.

Gaitan, D.F., L.A. Crum, C.C. Church, and R.A. Roy, Sonoluminescence and bubble dynamics from a single, stable, cavitaion bubble, J. Acoust. Soc. Am., 91, 3166-, 1991.

Gardon, R., The emissivity of transparent materials, Journal of the American Ceramics Society, 39, 278-287, 1956.

Garten, V.A., and R.B. Head, Crystalloluminescence and the nature of the critical nucleus, Phil. Mag., 8, 1793-1803, 1963.

Garten, V.A., and R.B. Head, Homogeneous nucleation in aqueous solutions, J. Cryst. Growth, 6, 349-351, 1970.

Geelhood, B.G., Impact of $40_{\mathrm{K}}$ on DUMAND; unwanted light in the ocean, in Proceedings of the 1982 DUMAND Signal Processing Workshop, edited by A. Roberts, pp. 30-56, University of Hawaii, Honolulu, Hawaii, 1982.

Gibbon, M.A., H. Sopp, J. Swanson, and A.J. Walton, Image intensifier studies of crystalloluminescence of NaCl, J. Phys. C.: Solid State Phys., 21, 1921-1934, 1988.

Ginster, U., M.J. Mottl, and R.P. Von Herzen, Heat flux from black smokers on the Endeavour and Cleft segments, Juan de Fuca Ridge, J. Geophys. Res., 99 (B3), 4937-4950, 1994.

Goldsborough, R.G., B. Allen, R. Stokey, and R.W. Simpson, 'High-Res' Electronic Still Camera, in Sea Technology, pp. 65-75, 1998.

Gregg, T.K.P., D.J. Fornari, M.R. Perfit, R.M. Haymon, and J.H. Fink, Rapid emplacement of a mid-ocean ridge lava flow on the East Pacific Rise at $9^{\circ} 41^{\prime}-51^{\prime}$ N, Earth and Planetary Science Letters, 144, E1-E8, 1996.

Hale, G.M., and M.R. Querry, Optical constants of water in the $200-\mathrm{nm}$ to $200-\mu \mathrm{m}$ wavelength region, Applied Optics, 12, 555-563, 1973.

Harvey, E.N., Sonoluminescence and sonic chemiluminescence, J. Am. Chem. Soc., 61, 2392-2398, 1939.

Haymon, R.M., Growth history of hydrothermal black smoker chimneys, Nature, 301, 694-698, 1983.

Haymon, R.M., D.J. Fornari, M.H. Edwards, S. Carbotte, D. Wright, and K.C. Macdonald, Hydrothermal vent distribution along the East Pacific Rise crest $\left(9^{\circ} 09^{\prime}\right.$ $54^{\prime} \mathrm{N}$ ) and its relationship to magmatic and tectonic processes on fast-spreading mid-ocean ridges, Earth and Planetary Science Letters, 104, 513-534, 1991. 
Haymon, R.M., D.J. Fornari, K.L. Von Damm, M.D. Lilley, M.R. Perfit, J.M. Edmond, W.C. Shanks III, R.A. Lutz, J.M. Grebmeier, S. Carbotte, D. Wright, E. McLaughlin, M. Smith, N. Beedle, and E. Olson, Volcanic eruptio of the midocean ridge along the East Pacific Rise crest at $9^{\circ} 45-52^{\prime} \mathrm{N}$ : Direct submersible observations of seafloor phenomena associated with an erkupton event in April, 1991, Earth and Planetary Science Letters, 119, 85-101, 1993.

Hekinian, R., J. Francheteau, V. Renard, R.D. Ballard, P. Choukroune, J.L. Cheminee, F. Albarede, J.F. Minster, J.L. Charlou, J.C. Marty, and J. Boulegue, Intense hydrothermal activity at the axis on the East Pacific Rise near $13^{\circ} \mathrm{N}$ : submersible witnesses the growth of sulfide chimney, Marine Geophysical Researches, 6, 1-14, 1983.

Hessler, R.R., and V.A. Kaharl, The deep-sea hydrothermal vent community, in Seafloor Hydrothermal Systems: Physical, Chemical, Biological, and Geological Interactions, edited by S.E. Humphris, R.A. Zierenberg, L.S. Mullineaux, and R.E. Thomson, AGU, Washington, DC, 1995.

Hiller, R., S.J. Putterman, and B.P. Barber, Spectrum of synchronous picosecond sonoluminescence, Phys. Rev. Lett., 69 (8), 1182-1184, 1992.

Jannasch, H., Microbial interactions with hydrothermal fluids, in Seafloor Hydrothermal Systems: Physical, Chemical, Biological, and Geological Interactions, edited by S.E. Humphris, R.A. Zierenberg, L.S. Mullineaux, and R.E. Thomson, AGU, Washington, DC, 1995.

Jarman, P., Sonoluminscence: A discussion, J. Acoust. Soc. Am., 32 (11), 1459-1462, 1960.

Jelley, J.V., Cerenkov Radiation and its Applications, 1958.

Jerlov, N.G., Optical Oceanography, 194 pp., Elsevier Publishing, co., Amsterdam, the Netherlands, 1968.

Johnson, H.P., and M.L. Holmes, Evolution in plate tectonics; The Juan de Fuca Ridge, in The Easter Pacific Ocean and Hawaii, edited by E.L. Winterer, D.M. Hussong, and R.W. Decker, pp. 73-91, Geological Society of America, Boulder, CO, 1989.

Kadko, D., and W. Moore, Radiochemical constraints on the crustal residence time of submarine hydrothermal fluids: Endeavour Ridge, Geochim. Cosmochim. Acta, 52, 659-668, 1988.

Karsten, J.L., S.R. Hammond, E.E. Davis, and R.G. Currie, Detailed geomorphology and neotectonics of the Endeavour Segment, Juan de Fuca Ridge: New results from Seabeam swath mapping, Geological Society of America Bulletin, 97, 213-221, 1986.

Klitgord, K.D., and J. Mammerickx, Northern East Pacific Rise: magnetic anomaly and bathymetric framework, Journal of Geophysical Research, 87, 6725-6750, 1982.

Koski, R.A., I.R. Jonasson, D.C. Kadko, V.K. Smith, and F.L. Wong, Compositions, growth mechanisms, and temporal relations of hydrothermal sulfide-sulfate-silica chimneys at the northern Cleft segment, Juan de Fuca Ridge, Journal of Geophysical Research, 99 (B3), 4813-4832, 1994. 
Kou, L., D. Labrie, and P. Chylek, Refractive indices of water and ice in the 0.65 to 2.5 $\mu \mathrm{m}$ spectral range., Applied Optics, 32, 3531-3540, 1993.

Kullenberg, G., Observed and computed scattering functions, in Optical Aspects of Oceanography, edited by N.G. Jerlov, and E.S. Nielsen, Academic Press, London, 1974.

Larson, R.L., Near-bottom geologic studies of the East Pacific Rise crest, Geological Society of America Bulletin, 82, 823-841, 1971.

LITE Workshop Participants, Light in Thermal Environments, pp. 44, Woods Hole Oceanographic Institution, Woods Hole, MA, 1993.

Little, S.A., K.D. Stolzenbach, and G.M. Purdy, The sound field near hydrothermal vents on Axial Seamount, Juan de Fuca Ridge, J. Geophys. Res., 95 (B8), 12,927$12,945,1990$.

McDuff, R.E., and J.R. Delaney, Periodic variability in fluid temperature at a seafloor hydrothermal vent, EOS, AGU Trans., 76, F710, 1995.

McLean, I., Electronic Imaging in Astronomy: Dectectors and Instrumentation, 472 pp., John Wiley \& Sons, Chichester, England, 1997.

McMahon, H.O., Thermal radiation from partically transparent reflecting bodies, Journal of the Optical Society of America, 40, 376-380, 1950.

Morel, A., Optical properties of pure water and pure sea water, in Optical Aspects of Oceanography, edited by N.G. Jerlov, and E.S. Nielsen, Academic Press, London, 1974.

Nelson, D.M., Photographic spectra of tribo-luminescence, J. Opt. Soc. Am., 12, 207215, 1926.

Nicodemus, F.E., Radiometry, in Applied Optics and Optical Engineering, edited by R. Kingslake, pp. 263-308, Academic Press, New York, 1967.

O'Neill, P.J., R.N. Jinks, E.D. Herzog, B.-A. Battelle, L. Kass, G.H. Renninger, and S.C. Chamberlain, The morphology of the dorsal eye of the hydrothermal vent shrimp, Rimicaris exoculata, Visual Neurocience, 12, 861-875, 1995.

Oosting, S.E., and K.L. Von Damm, Bromide/chloride fractionation in seafloor hydrothermal fluids from $9-10^{\circ} \mathrm{N}$ East Pacific Rise, Earth and Planetary Science Letters, 144, 133-145, 1996.

Overmann, J., H. Cypiowka, and N. Phennig, An extremely low-light-adapted phototrophic sulfur bacteria from the Black Sea, Limnology and Oceanography, 370, 150-155, 1992.

Palmer, K.F., D. Williams, Optical properties of water in the near infrared, Journal of the Optical Society of America, 64, 1107-1110, 1974.

Pegau, W.S., D. Grey, and J.R. Zaneveld, Absorption and attenuation of visible and nearinfrared light in water: dependence on temperature and salinity, Applied Optics, 36, 6035-6046, 1997.

Pelli, D.G., and S.C. Chamberlain, The visibility of $350^{\circ} \mathrm{C}$ black-body radiation by the shrimp Rimicaris exoculata and man, Nature, 337, 460, 1989. 
Pfennig, N., General physiology and ecology of photosynthetic bacteria, in The Photosynthetic Bacteria, edited by R.K. Clayton, and W.R. Sistrom, Plenum Press, New York, 1978.

Pope, D.G., and E.S. Fry, Absorption spectrum (380-700 nm) of pure water. II. Integrating cavity measurements, Applied Optics, 36, 8710-8723, 1997.

Radziminski, C.Z., A.S. Acosta, J.I. Zink, and C.L. Van Dover, Report of Photoluminescence Work, Unpublished, 1997.

Rea, D.K., Asymmetric sea-floor spreading and a nontransform axis offset: The East Pacific Rise $20^{\circ} \mathrm{S}$ survey area, Geological Society of America Bulletin, 89, 836$844,1978$.

Reynolds, G.T., Light and life at hydrothermal vents, pp. 19, Princeton University, Princeton, NJ, 1995.

Reynolds, G.T., Evidence for vapor bubble luminescence, pp. 6, Princeton University, Princeton, NJ, 2000.

Reynolds, G.T., A.J. Walton, and S.M. Gruner, Observations of sonoluminescence using image intensification, Rev. Sci. Instrum., 53 (11), 1673-1676, 1982.

Riedesel, M., J.A. Orcutt, K.C. Macdonald, and J.S. McClain, Microearthquakes in the black smoker hydrothermal field, East Pacific Rise at $21^{\circ} \mathrm{N}, J$. Geophys. Res., 87 (B13), 10,613-10,623, 1982.

Roberts, A., Potassium 40 in the ocean and how to live with it, in DUMAND 78, edited by A. Roberts, pp. 139-148, University of Hawaii, Honolulu, Hawaii, 1979.

Rona, P.A., G. Klinkhammer, T.A. Nelsen, J.H. Trefry, and H. Elderfield, Black smokers, massive sulphides and vent biota at the Mid-Atlantic Ridge, Nature, 321, 33-37, 1986.

Schultz, A., and H. Elderfeld, Controls on the physics and chemistry of seafloor hydrothermal circulation, Phil. Trans. R. Soc. Lond. A, 355, 387-425, 1997.

Sehgal, C., R.P. Steer, R.G. Sutherland, and R.E. Verral, Sonoluminescence of argon saturated alkali metal salt solutions as a probe of acoustic cavitation, J. Chem. Phys., 70 (5), 2242-2248, 1979.

Smith, M.O., and J.R. Delaney, Variability of emitted radiation from two hydrothermal vents, EOS Transactions, $A G U, 70,70,1989$.

Smith, M.O., and J.R. Delaney, Spatial and temporal variation of emitted visible and near infrared radiation from two hydrothermal vents, , unpublished.

Smith, R.C., and K.S. Baker, Optical properties of the clearest natural waters (200-800 nm), Applied Optics, 20, 177-184, 1981.

Stein, C.A., and S. Stein, Constraints of hydrothermal heat flux through the oceanic lithosphere from global heat flow, J. Geophys. Res., 99 (B2), 3081-3095, 1994.

Sullivan, S.A., Experimental study of the absorption in distilled water, artificial sea water, and heavy water in the visible region of the spectrum, Journal of the Optical Society of America, 53, 962-968, 1963. 
Suslick, K.S., The chemical effects of ultrasound, in Scientific American, pp. 80-86, 1989.

Suslick, K.S., Sonochemistry, Science, 247, 1439-1445, 1990.

Tapley, D.W., Sulfide-dependent oxidative stress in marine invertebrates, especially thiotrophic symbioses, Doctoral thesis, University of Maine, Orono, Maine, 1993.

Tapley, D.W., G.R. Buettner, and J.M. Shick, Free radical and chemiluminescence as products of the spontaneous oxidation of sulfide in seawater, and their biological implications, Biological Bulletin, 196, 52-56, 1999.

Taylor, K.J., and P.D. Jarman, The spectra of sonoluminescence, Aust. J. Phys., 23, 319-334, 1970.

Tivey, M.K., Modeling chimney growth and associated fluid flow at seafloor hydrothermal vent sites, in Seafloor Hydrothermal Systems: Physical, Chemical, Biological, and Geological Interactions, edited by S.E. Humphris, R.A. Zierenberg, L.S. Mullineaux, and R.E. Thomson, AGU, Washington, D. C., 1995.

Tivey, M.K., and J.R. Delaney, Growth of large sulfide structures on the Endeavour Segment of the Juan de Fuca Ridge, Earth and Planetary Science Letters, 77, 303$317,1986$.

Tivey, M.K., and R.E. McDuff, Mineral precipitation in the walls of black smoker chimneys: a quantitative model of transport and chemical reactions, J. Geophys. Res., 95 (B8), 12,617-12,637, 1990.

Tomczak, M., and J.S. Godfrey, Regional Oceanography: An Introduction, Pergamon, London, 1994.

Tyson, J.A., Low-light-level charge-coupled device imaging in astronomy, Journal of the Optical Society of America, 3, 2131-2138, 1986.

Tyson, J.A., Progress in low-light-level charge-coupled device imaging in astronomy, Journal of the Optical Society of America, 7, 1231-1236, 1990.

Valiela, I., Marine Ecological Processes, Springer-Verlag, New York, 1995.

Van de Hulst, H.C., Light Scattering by Small Particles, John Wiley \& Sons, Inc., New York, 1957.

Van Dover, C.L., The Ecology of Deep-Sea Hydrothermal Vents, 424 pp., Princeton University Press, Princeton, N. J., 2000.

Van Dover, C.L., J.R. Cann, C. Cavanaugh, S.C. Chamberlain, J.R. Delaney, D. Janecky, J. Imhoff, J.A. Tyson, and L.W. Participants, Light at deep sea hydrothermal vents, $E O S, 75,44-45,1994 a$.

Van Dover, C.L., G.T. Reynolds, A.D. Chave, and J.A. Tyson, Light at deep-sea hydrothermal vents, Geophysical Research Letters, 23 (16), 2049-2052, 1996.

Van Dover, C.L., E.Z. Szuts, S.C. Chamberlain, and J.R. Cann, A novel eye in "eyeless" shrimp from hydrothermal vents of the Mid-Atlantic Ridge, Nature, 337, 458-460, 1989. 
Vazquez, F., J.-Z. Zhang, and F.J. Millero, Effect of metals on the rate of the oxidation of $\mathrm{H}_{2} \mathrm{~S}$ in seawater., Geophysical Research Letters, 16 (12), 1363-1366, 1989.

Von Damm, K.L., Chemistry of submarine hydrothermal solutions at $21^{\circ}$ North, East Pacific Rise and Guaymas Basin, Gulf of California, Doctoral Dissertation thesis, MIT/WHOI Joint Program, Woods Hole/Cambridge, MA, 1983.

Von Damm, K.L., S.E. Oosting, R. Kozlowski, L.G. Buttermore, D.C. Colodner, H.N. Edmonds, J.M. Edmond, and J.M. Grebmeier, Evolution of East Pacific Rise hydrothermal vent fluids following a volcanic eruption, Nature, 375, 47-50, 1995a.

Von Damm, K.L., Controls on the chemistry and temporal variability of seafloor hydrothermal fluids, in Seafloor Hydrothermal Systems: Physical, Chemical, Biological, and Geological Interactions, edited by S.E. Humphris, R.A. Zierenberg, L.S. Mullineaux, and R.E. Thomson, AGU, Washington, DC, 1995 b.

Walton, A.J., Triboluminescence, Advances in Physics, 26, 887-948, 1977.

Walton, A.J., and G.T. Reynolds, Sonoluminescence, Advances in Physics, 33, 595-660, 1984.

Weninger, K.R., C.G. Camara, and S.J. Putterman, Energy focusing in a converging fluid flow: Implications for sonoluminescence, Phys. Rev. Lett., 83 (10), 20812084, 1999.

White, S.N., J.W. Bailey, C.L. Van Dover, and A.D. Chave, Measurements of light at hydrothermal vents, RIDGE Events, 7 (2), 10-12, 1996a.

White, S.N., A.D. Chave, J.W. Bailey, C.L. Van Dover, and G.T. Reynolds, Measurements of light at hydrothermal vents, $9^{\circ} \mathrm{N}$ East Pacific Rise, EOS Transactions, $A G U, 77, \mathrm{~F} 404,1996 \mathrm{~b}$.

White, S.N., A.D. Chave, G.T. Reynolds, E.J. Gaidos, J.A. Tyson, and C.L. Van Dover, Variations in ambient light emission from black smokers and flange pools on the Juan de Fuca Ridge, Geophys. Res. Lett., 27 (8), 1151-1154, 2000.

Williams, A.B., and P.A. Rona, Two new caridean shrimps (Bresiliidae) from a hydrothermal field on the Mid-Atlantic Ridge, Journal of Crustacean Biology, 6 , 446-462, 1986.

Wolfe, W.L., and G.J. Zissis, The Infrared Handbook, Infrared Information Analysis Center, 1989.

Wolken, J.J., Light Detectors, Photoreceptors, and Imaging Systems in Nature, Oxford University Press, Oxford, 1995.

Yasui, K., Single-bubble and multiple bubble sonoluminescence, Phys. Rev. Lett., 83 (21), 4297-4300, 1999.

Yurkov, V., and J.T. Beatty, Isolation of aerobic anoxygenic photosynthetic bacteria from black smoker plume waters of the Juan de Fuca Ridge in the Pacific Ocean, Appl. Environ. Microbiol., 64, 337-341, 1998.

Zink, J., Squeezing light out of crystals: triboluminescence, Naturwiss., 68, 507-512, 1981. 
Zink, J.I., and B.P. Chandra, Light emission during growth and destruction of crystals. Crystalloluminescence and triboluminescence, Journal of Physical Chemistry, 86, 5-7, 1982. 


\section{BIOGRAPHICAL Note}

I was born in Dallas, Texas on March 13, 1971. While I was always interested in science, my early focus was on space exploration. This led me to Purdue University (following my father's footsteps) where I majored in Aeronautical and Astronautical Engineering (B.S.A.A.E. 1993). While at Purdue, I worked as a co-operative education student at NASA - Johnson Space Center in Houston, TX. Some geology classes in college renewed in me a long-buried interest in earth science. That renewed interest (and a NASA hiring freeze) led me to apply to graduate school in Marine Geology \& Geophysics.

The MIT/WHOI Joint Program in Oceanography combined my interests in science, exploration, and hands-on engineering. My graduate research included electromagnetic methods, analysis of side-scan sonar data, and investigating light emission at deep-sea hydrothermal vents. I also participated in four oceanographic cruises and six DSV Alvin dives.

\section{Research Cruise Experience}

Shipboard scientist on four research cruises to investigate mid-ocean ridge hydrothermal systems using manned submersibles and unmanned tethered vehicles. Maintained instruments to measure ambient light at vents; interfaced instruments with the submarine; trained science observers in use of instruments; collected and analyzed water samples; collected and sorted biological specimens; participated as an observer on DSV Alvin six submersible dives.

June/July 1998

November/December 1997

April 1996

August/September 1995
$R / V$ Atlantis

$R / N$ Atlantis

$R / V$ Atlantis II

DSVSS Laney Chouest
Juan de Fuca Ridge

$9^{\circ} \mathrm{N}$ East Pacific Rise

$9^{\circ}$ N East Pacific Rise

Juan de Fuca Ridge

\section{Publications}

White, S. N., A. D. Chave, G. T. Reynolds, E. J. Gaidos, J. A. Tyson, and C. L. Van Dover, Variations in ambient light emission from black smokers and flange pools on the Juan de Fuca Ridge, Geophys. Res. Lett., 27: 1151-1154, 2000.

White, S. N., S. E. Humphris, and M. C. Kleinrock, New observations on the distribution of past and present hydrothermal activity in the TAG area of the MidAtlantic Ridge ( $26^{\circ} 08^{\prime}$ N), Mar. Geophys. Res., 20:41-56 (and Erratum 20;139), 1998.

White, S. N., A. D. Chave, and J. H. Filloux, A look at galvanic distortion in the Tasman Sea and the Juan de Fuca Plate, J. Geomag. Geoelectr., 49:1373-1386, 1997. 